\title{
Poly(trimethylene carbonate)-based composites for biomedical applications
}

Zhengchao Guo 
Poly(trimethylene carbonate)-based composites for biomedical applications

\section{ZHENGCHAO GUO}


Poly(trimethylene carbonate)-based composites for biomedical applications

Zhengchao Guo

$\mathrm{PhD}$ thesis with references and summaries in English, Dutch and Chinese

University of Twente, Enschede, The Netherlands

November 2020

The research described in this thesis was carried out between September 2014 to March 2019 in the research group Biomaterials Science and Technology (BST), Faculty of Science and Technology (TNW), University of Twente, Enschede, The Netherlands.

A personal grant for Zhengchao Guo was obtained from the Chinese Scholarship Council (CSC).

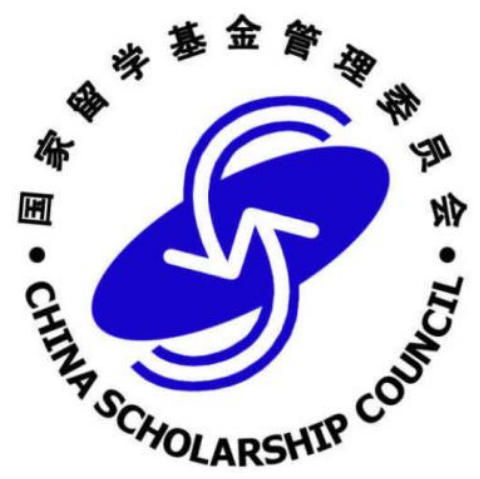

The printing of this thesis was sponsored by:

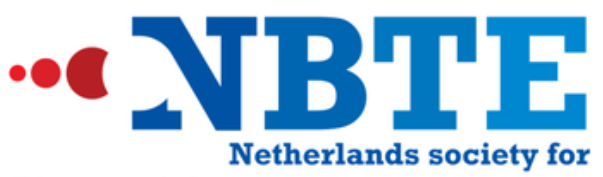
Biomaterials and Tissue Engineering 


\title{
POLY(TRIMETHYLENE CARBONATE)-BASED COMPOSITES FOR BIOMEDICAL APPLICATIONS
}

\section{DISSERTATION}

\author{
to obtain \\ the degree of doctor at the University of Twente, \\ on the authority of the rector magnificus, \\ Prof. Dr. T.T.M. Palstra, \\ on account of the decision of the Doctorate Board \\ to be publicly defended \\ on Friday the $20^{\text {th }}$ of November 2020 at 10.45
}

by

\section{Zhengchao Guo}

born on $18^{\text {th }}$ of July 1988

in Anyang, China 
This dissertation has been approved by:

Supervisor:

Prof. Dr. D.W. Grijpma

Co-supervisor:

Dr. A.A. Poot

(C) 2020 Zhengchao Guo. All rights reserved. No parts of this thesis may be reproduced, stored in a retrieval system or transmitted in any form or by any means without permission of the author.

ISBN: 978-90-365- 5074-1

DOI: $10.3990 / 1.9789036550741$ 


\section{Graduation Committee:}

Chairman / secretary

Supervisor:

Co-supervisor:

Committee Members:
Prof. Dr. J.L. Herek

Prof. Dr. D.W. Grijpma

Dr. A.A. Poot

Prof. Dr. J. Seppälä

Aalto University, The Finland

Prof. dr. P.J. Dijkstra

Soochow University, China

Prof. Dr. G.J. Vancso

University of Twente, The Netherlands

Prof. Dr. D. Stamatialis

University of Twente, The Netherlands

Dr. J.C.H. Leijten

University of Twente, The Netherlands 



\section{Publication list}

Published

Chapter 3. S.G. Rotman, Z. Guo, D.W. Grijpma, A.A. Poot, Preparation and characterization of poly(trimethylene carbonate) and reduced graphene oxide composites for nerve regeneration, Polym. Adv. Technol., 28 (2017) 1233-1238.

Chapter 5. Z. Guo, S. Kofink, H. Chen, J. Liang, D.W. Grijpma, A.A. Poot, Synthesis and characterization of rGO-graft-poly(trimethylene carbonate) for nerve regeneration conduits, Biomed. Mater., 14 (2019) 034101.

Chapter 6. Z. Guo, J. Liang, M.J. Ankone, A.A. Poot, D.W. Grijpma, H. Chen, Fabrication of poly(trimethylene carbonate)/reduced graphene oxide-graft-poly(trimethylene carbonate) composite scaffolds for nerve regeneration, Biomed. Mater., 14 (2019) 024104.

Chapter 7. Z. Guo, D.W. Grijpma, A.A. Poot, Leachable poly(trimethylene carbonate)/CaCO $\mathrm{C}_{3}$ composites for additive manufacturing of micro-porous vascular structures, Materials., 13 (2020) 3435 .

Chapter 8. Z. Guo, D.W. Grijpma, A.A. Poot, Preparation and characterization of flexible and elastic porous tubular PTMC scaffolds for vascular tissue engineering, Polym. Adv. Technol., 28 (2017) 1239-1244.

\section{Submitted}

Chapter 2. Z. Guo, D.W. Grijpma, A.A. Poot, Advanced polymer-based composites and Structures for Biomedical Applications, Eur. Polym. J., (2020)

\section{Other}

H. Gojzewski, Z. Guo, W. Grzelachowska, M.G. Ridwan, M.A. Hempenius, D.W. Grijpma, G.J. Vancso, Layer-by-layer printing of photopolymers in 3D: How weak is the interface?, ACS Appl. Mater. Interfaces, 12 (2020) 8908-8914.

G. Weisgrab, O. Guillaume, Z. Guo, P. Heimel, P. Slezak, A.A. Poot, D.W. Grijpma, A. Ovsianikov. 3D Printing of large-scale and highly porous biodegradable tissue engineering scaffolds from poly(trimethylene-carbonate) using two-photon-polymerization, Biofabrication, 12 (2020) 045036. 



\section{Contents}

Chapter 1 General introduction 1

Chapter 2 Advanced polymer-based composites and structures for 9 biomedical applications

Chapter 3 Preparation and characterization of poly(trimethylene carbonate) and reduced graphene oxide composites for nerve regeneration

Chapter 4 Evaluation of a PTMC/rGO composite nerve guide conduit: An in vivo pilot study

Chapter 5 Synthesis and characterization of rGO-graft-poly(trimethylene carbonate) for nerve regeneration conduits

Chapter 6 Fabrication of poly(trimethylene carbonate)/reduced graphene oxide-graft-poly(trimethylene carbonate) composite scaffolds for nerve regeneration

Chapter 7 Leachable poly(trimethylene carbonate) $/ \mathrm{CaCO}_{3}$ composites for additive manufacturing of micro-porous vascular structures

Chapter 8 Preparation and characterization of flexible and elastic porous tubular PTMC scaffolds for vascular tissue engineering

Appendix Dynamic culturing of smooth muscle cells in porous tubular poly(trimethylene carbonate) scaffolds

Summary

and outlook

Samenvatting 



\section{Chapter 1 - General introduction}

Biomedical engineering has been steadily developing in the past decades because of the increase of human life expectancy [1]. Aging, diseases and damage to tissues and organs caused by e.g. trauma has shown large progress in replacement technologies, drug delivery systems and regeneration therapies to cure patients. Tissue engineering and regenerative medicine are technologies that were proposed about 30 years ago [2]. These techniques use biomaterials and specific human cells to engineer functional devices and native tissue-like implants for biomedical applications. Using this concept, patients may be cured in an efficient and patient friendly way without the necessity to use medical devices. Traditionally, tissue engineering includes cells (preferably isolated from the patient), fabrication of a biodegradable porous scaffold, seeding and culturing the cells in the scaffold, implantation of the construct [3]. The scaffold as a temporal support for cell adhesion is incredibly important. The cells, which can either be added to the scaffold or attracted from the surrounding tissue, should create a new functional extracellular matrix [4].
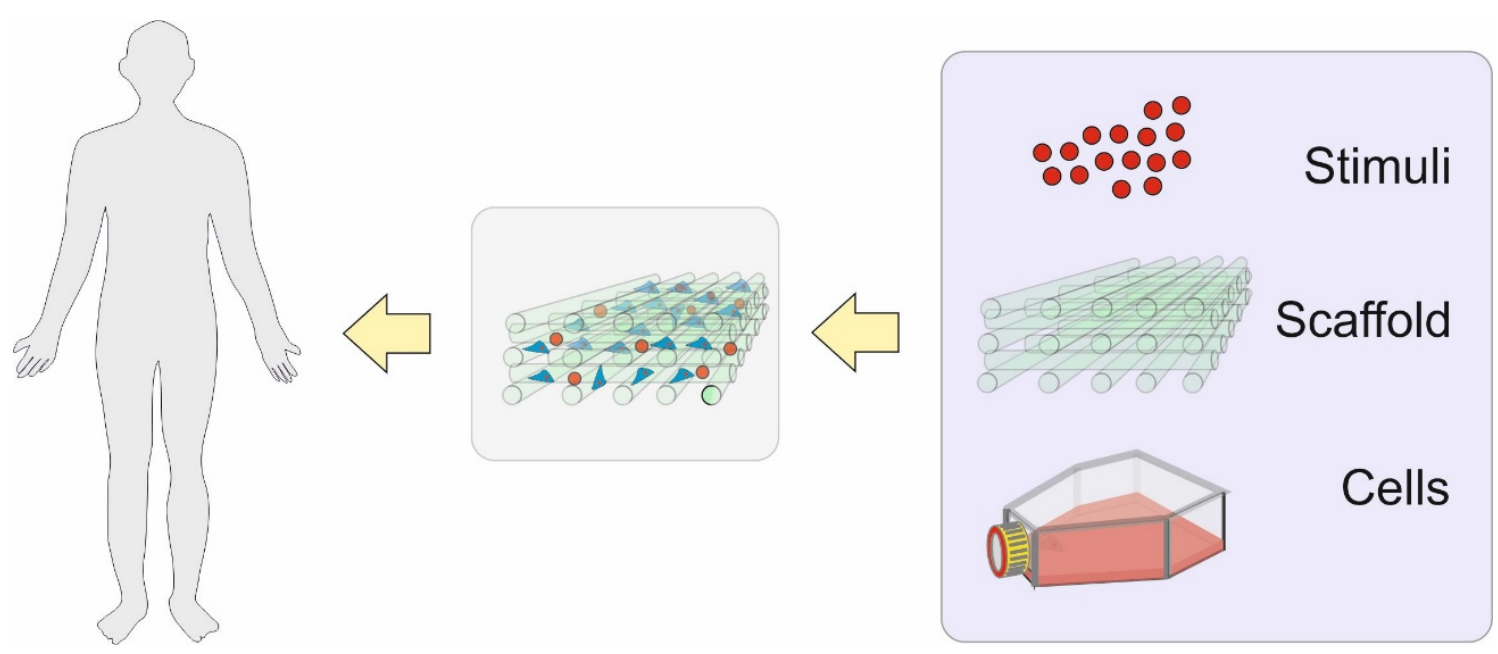

Figure 1. General scheme of tissue engineering

To achieve proper regeneration of tissue, scaffolds can be made functional, bioactive or even stimuli-responsive and are preferably prepared from biodegradable polymers [5]. These materials are generally based on polymers which can be of natural or synthetic origin. The 
latter polymers do not instruct cells, but serve as a temporary support and thus may not lead to a functional tissue in a short time. The use of polymer-based composites is a promising strategy to increase tissue formation and regeneration [6]. For example, electrically conductive materials in a polymer matrix may stimulate the outgrowth of nerve cells resulting in functional axons [7]. Another example is the use of polymer/hydroxyapatite composites which can regenerate bone [8]. In addition, composite fillers can be used for oxygen supply and can provide magnetic and antimicrobial properties [9].

Biodegradable polymers (synthetic and natural) have frequently been used to fabricate scaffolds for tissue engineering and regenerative medicine [10]. Poly(trimethylene carbonate) (PTMC) as an amorphous polymer with rubber-like properties has been studied for various biomedical applications. One of the advantages of PTMC as a biomaterial is its flexibility, making it highly suitable for soft tissue engineering [11]. The material degrades by surface erosion, in adjustable times from a few months to over years, allowing PTMC scaffolds to retain their mechanical properties during long periods of time [12].

Manufacturing techniques determine to a large extent the specific porous structure and surface topography of scaffolds [13]. Porous tissue engineering scaffolds can be fabricated by particle leaching, electrospinning and additive manufacturing techniques. The use of polymer-based composites may provide new biomedical implants adapted to the intended application.

\section{Scope of the study}

A series of studies described in this thesis were directed towards the development of different types of PTMC-based composites for the fabrication of peripheral nerve guide conduits and vascular grafts. PTMC was selected because of its biocompatibility, biodegradability as well as flexible and elastic properties, making it highly suitable for the manufacturing of scaffolds for nerve and blood vessel regeneration.

To replace damaged or diseased peripheral nerves, conduits that guide and enhance nerve regeneration can be constructed from electrically conductive materials. Such guides were made from PTMC-based composites by incorporating reduced graphene oxide ( $\mathrm{rGO}$, a conductive filler) in the polymer matrix. The synthesis of the components and material properties of (non)porous PTMC/rGO composite films and conduits were evaluated. The structures were prepared by solvent casting and photo-crosslinking. The conduits were made porous by $\mathrm{NaF}$ 
particle leaching. An in vivo model, i.e. bridging of a sciatic nerve in the rabbit, was used to study the potential for nerve regeneration.

To optimize the compatibility of rGO in the PTMC polymer matrix, rGO-graft-PTMC was synthesized. PTMC/rGO-graft-PTMC films and porous mats were manufactured by solvent casting and electrospinning, respectively. The material properties of the composite structures were evaluated and their biocompatibility was tested by culturing with PC-12 rat neuronal cells.

Porous tubular scaffolds hold promise for vascular tissue engineering. Designed capillary structures were built by stereolithography using a $\mathrm{PTMC} / \mathrm{CaCO}_{3}$ particle composite, followed by leaching of the particles. Larger porous tubular scaffolds were prepared by solvent casting of a PTMC/NaCl composite, photo-crosslinking and leaching of the salt particles. Human smooth muscle cells were seeded in the scaffolds, after which the constructs were subjected to pulsatile flow conditions in a bioreactor.

\section{Outline of the thesis}

In Chapter 2 a review is presented on polymer-based composites with functional properties in relation to their intended biomedical application. In the first part the review is focused on polymer-based composites containing bioactive particles/fillers, like ceramic, electrically conductive, magnetic, oxygen-generating and antibacterial materials. In the second part the review is focused on non-bioactive polymer-based composites, containing reinforcing fibers and leachable particles. In the final part, the review is directed towards the manufacturing of 3D structures from polymer-based composites, using particle leaching, electrospinning and additive manufacturing. These techniques are currently widely applied for the construction of advanced biomedical scaffolds and implants for tissue regeneration. 


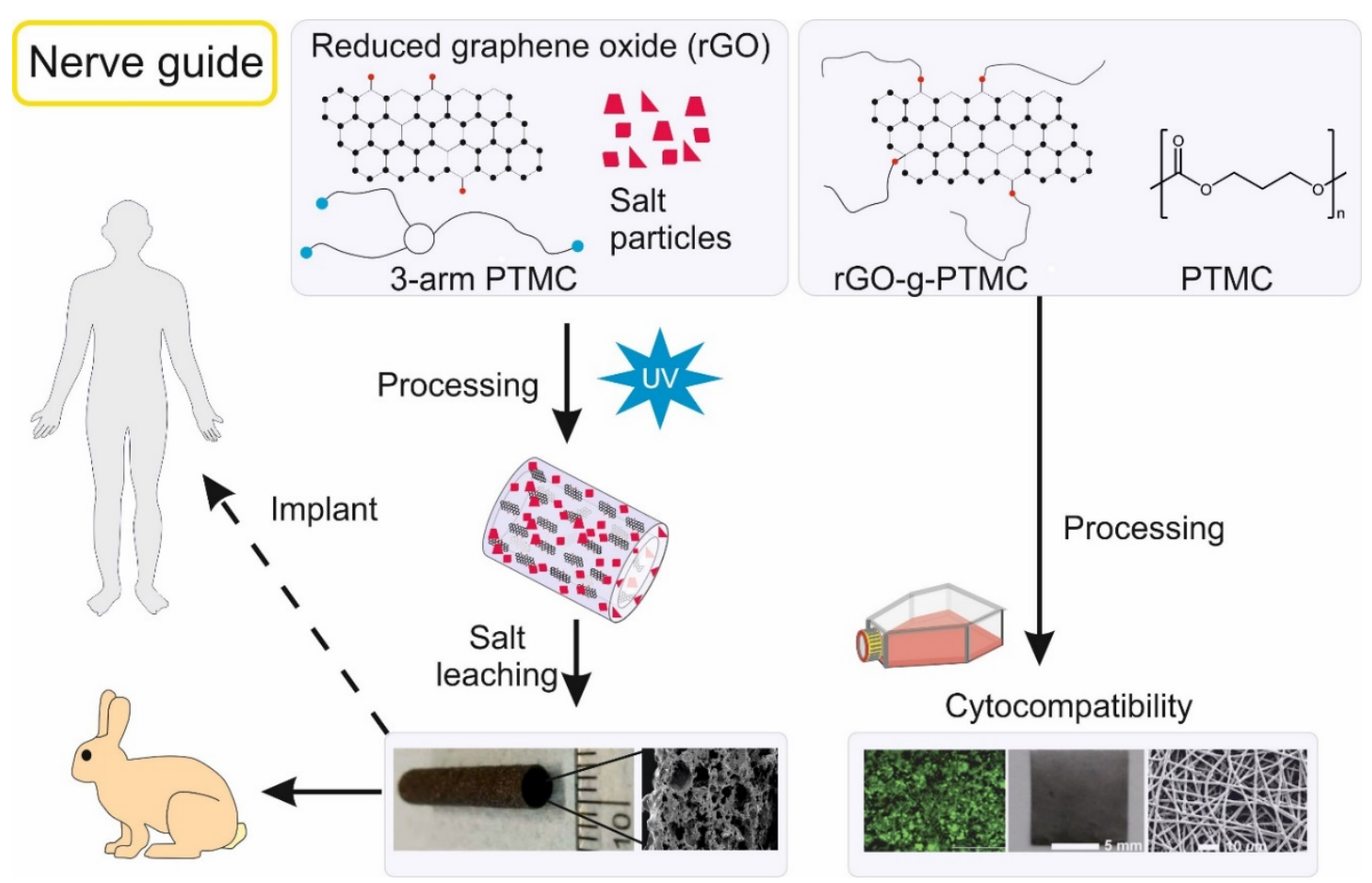

Figure 2. Overview of studies on the tissue engineering of a nerve guide

In Chapter 3 a polymer-based composite for the preparation of a nerve guide channel (NGC) for nerve regeneration is discussed. Because electrically conductive NGCs promote axon regeneration, a composite material composed of PTMC and a conductive filler, reduced graphene oxide (rGO), was studied. Three-armed low molecular weight PTMC endfunctionalized with methacrylate groups was selected because of its biocompatibility, biodegradability, flexibility and crosslinkability. rGO with a high aspect ratio was prepared and incorporated in the PTMC matrix. The content of rGO in the PTMC matrix was varied in order to determine the threshold amount for electrical conductivity. Biocompatibility of photocrosslinked PTMC/rGO composite films with respect to PC12 neuronal cells was evaluated.

In Chapter 4 a porous NGC made from a composite of three-armed methacrylated PTMC, rGO and NaF particles was studied. Dip coating, photo-crosslinking and particle leaching were used to generate porous tubular conduits. The PTMC/rGO composite scaffold was evaluated with regard to nerve regeneration in a rabbit model to bridge a $15 \mathrm{~mm}$ sciatic nerve gap, and compared to a PTMC NGC without the conductive rGO filler.

To prevent rGO aggregate formation due to van der Waals interactions in the preparation of PTMC/rGO composites, rGOs with grafted PTMC oligomers were evaluated as described in 
Chapter 5. Thermally stable hydroxyl groups on graphene sheets, prepared by nitrene chemistry, were used as initiator for the ring-opening polymerization of TMC. The physicochemical properties and electrical conductivity of the rGO-graft-PTMC materials were evaluated. The biocompatibility of photo-crosslinked PTMC/rGO-graft-PTMC composite films was investigated by culturing with PC12 neuronal cells.

In Chapter 6 we evaluated a biocompatible and flexible fibrous scaffold fabricated by electrospinning of a dispersion of linear high molecular weight PTMC and the rGO-graftPTMC. Loading of different amounts of rGO into the electrospun PTMC fibers, using the rGOgraft-PTMC, allowed to study the morphology and dimension of the fibers and hydrophilicity of the fibrous mats. The viability of PC12 cells seeded on the composite fibrous mats was investigated and compared to the viability of PC12 cells on electrospun fibrous mats of pure PTMC.

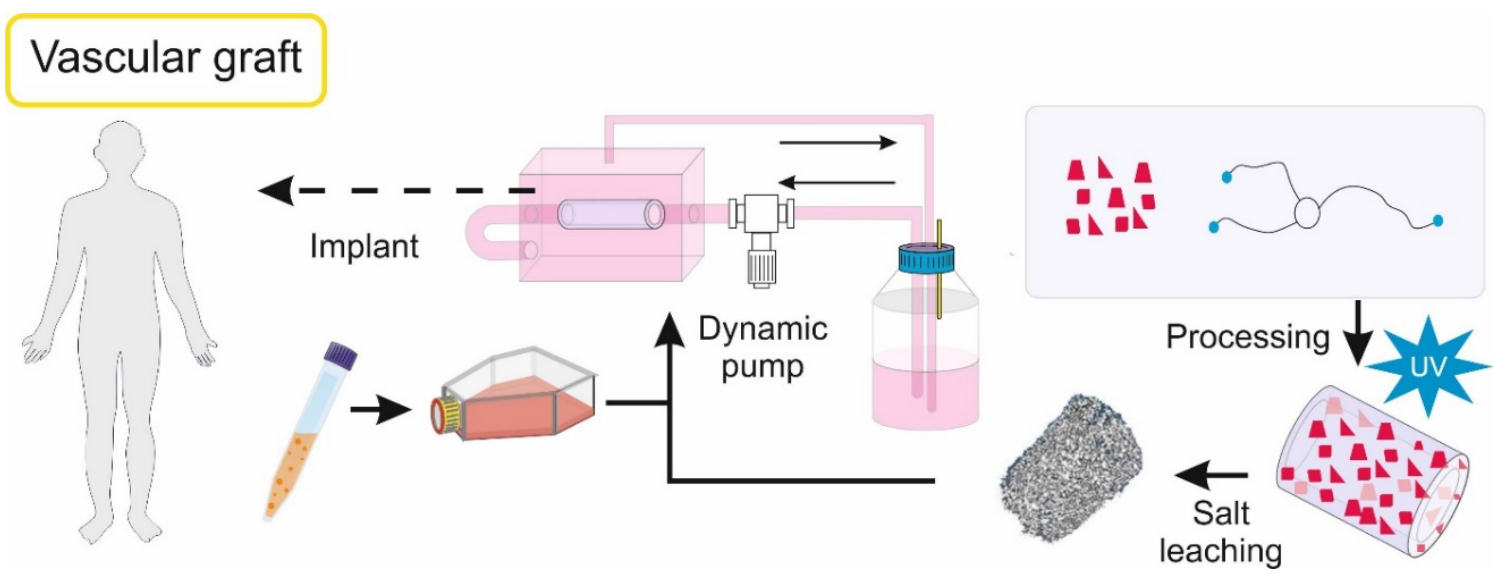

Figure 3. Overview of studies on the tissue engineering of a vascular graft

In Chapter 7 micro-porous scaffolds for vascular tissue engineering and organ models are described. Three-armed low molecular weight PTMC end-functionalized with methacrylate groups was combined with $\mathrm{CaCO}_{3}$ particles as porogen. The minimum amount of particles for complete leaching of photo-crosslinked composite films was determined. Moreover, the mechanical properties and intrinsic permeability of the resulting porous PTMC films were determined. Based on the results, a $\mathrm{PTMC} / \mathrm{CaCO}_{3}$ composite resin was formulated for the manufacturing of branched vascular channels by stereolithography. Upon leaching of the 
$\mathrm{CaCO}_{3}$ particles, micro-porous vascular structures were obtained with a high intrinsic permeability, indicating no mass transfer limitations in the case of cell seeding.

In Chapter 8 a fabrication method for the preparation of small-diameter vascular grafts is described. Three-armed methacrylate end-functionalized PTMC with different molecular weights was synthesized. A quartz glass mold was filled with a composite of PTMC macromer $/ \mathrm{NaCl}$ particles. Subsequently, the polymer was photo-crosslinked, the scaffold was demolded, and the particles were leached in water to give a porous tubular scaffold. The inner diameter, wall thickness, pore size and porosity of the scaffolds were evaluated. The mechanical properties of the scaffolds were characterized and compared to those of native blood vessels.

In the Appendix porous tubular scaffolds with suitable properties for vascular tissue engineering were prepared by the method described in Chapter 8. Culturing of human smooth muscle cells in the porous tubular scaffolds under static as well as dynamic conditions in a bioreactor was histologically evaluated. The mechanical properties of the constructs were determined and compared to those of native blood vessels.

\section{References}

[1] J.D. Bronzino, What is biomedical engineering? In: Introduction to Biomedical Engineering, Academic Press, (2012) 16-21.

[2] R. Langer, J.P. Vacanti, Tissue engineering, Science, 260 (1993) 920-926.

[3] R. Langer, D.A. Tirrell, Designing materials for biology and medicine, Nature, 428 (2004) 487-492.

[4] S.J. Hollister, Porous scaffold design for tissue engineering, Nat. Mater., 4 (2005) 518-524.

[5] A.J. Teo, A. Mishra, I. Park, Y.J. Kim, W.T. Park, Y.J. Yoon, Polymeric biomaterials for medical implants and devices, ACS Biomater. Sci. Eng., 2 (2016) 454-472.

[6] S. Ramakrishna, J. Mayer, E. Wintermantel, K. W. Leong, Biomedical applications of polymer-composite materials: a review, Compos. Sci. Technol., 61 (2001) 1189-1224. 
[7] Z. Zhang, M. Rouabhia, Z. Wang, C. Roberge, G. Shi, P. Roche, J. Li, L. H. Dao, Electrically conductive biodegradable polymer composite for nerve regeneration: electricitystimulated neurite outgrowth and axon regeneration, Artif. Organs, 31 (2007) 13-22.

[8] Y. Huang, J. Xu, D. Zhou, L. Xu, B. Zhao, Z. Li, Simultaneous reinforcement and toughening of polymer/hydroxyapatite composites by constructing bone-like structure, Compos. Sci. Technol., 151 (2017) 234-242.

[9] M. M. Zagho, E. A. Hussein, A.A. Elzatahry, Recent overviews in functional polymer composites for biomedical applications, Polymers, 10 (2018) 739.

[10] K. Rezwan, Q.Z. Chen, J.J. Blaker, A.R. Boccaccini, Biodegradable and bioactive porous polymer/inorganic composite scaffolds for bone tissue engineering, Biomaterials, 27 (2006) $3413-3431$.

[11] J.J. Rongen, B. van Bochove, G. Hannink, D.W. Grijpma, P. Buma, Degradation behavior of, and tissue response to photo-crosslinked poly(trimethylene carbonate) networks, J. Biomed. Mater. Res. A, 104 (2016) 2823-2832.

[12] Z. Zhang, R. Kuijer, S.K. Bulstra, D.W. Grijpma, J. Feijen, The in vivo and in vitro degradation behavior of poly(trimethylene carbonate), Biomaterials, 27 (2006) 1741-1748.

[13] P. Zhao, H. Gu, H. Mi, C. Rao, J. Fu, L. S. Turng, Fabrication of scaffolds in tissue engineering: a review, Front. Mech. Eng., 13 (2018) 107-119. 


\section{Chapter 2 - Advanced polymer-based composites and structures for biomedical applications}

Zhengchao Guo, André A. Poot, Dirk W. Grijpma.

Department of Biomaterials Science and Technology, Faculty of Science and Technology, University of Twente, Enschede, The Netherlands.

Submitted

Z. Guo, D. W. Grijpma, A. A. Poot, Advanced polymer-based composites and structures for biomedical applications, Eur. Polym. J., (2020) 


\begin{abstract}
A fast increasing demand of medical products based on biomaterials and tissue engineering has led to an extensive growth in biomedical research in the past two decades. A highly interesting class of biomaterials are polymer-based composites, which nowadays are widely used in biomedical applications due to their outstanding physical and mechanical properties. In this paper, we aim to summarize the advancement in polymer-based composites with regard to their properties, structure and fabrication using different techniques. Bioactive polymer-based composites, such as bone-forming, electrically conductive, magnetic, bactericidal and oxygenreleasing materials, as well as non-bioactive polymer-based composites containing reinforcing fillers and porogens are discussed. Amongst others, scaffold structures fabricated by particle leaching, electrospinning and additive manufacturing are described. In each section, significant and recent advances of polymer-based composites in biomedical applications are addressed.
\end{abstract}

\title{
1 Introduction
}

\subsection{Overview}

The biomaterials field has seen a strong growth in the past decades [1-4]. Biomaterials are used to prepare biomedical devices such as hydrogel contact lenses, polymer or metal stents, artificial heart valves, steel joint and hip replacements, knee and ligament implants, polymer vascular grafts, ceramic dental implants, polymer sutures, surgical adhesives, polymer barrier films, porous dialysis membranes, etc. [5-11]. Due to the fast increase of medical product demands caused by population expansion and aging, the numbers of both pre-clinical and clinical biomaterial studies are dramatically growing [12-14]. According to a global industry analysis report, the global biomaterials market was more than USD 94 billion in 2018 and is forecasted to be over USD 256 billion by 2025, i.e. an increase of more than $15 \%$ between 2019 and 2025 [15].

Instead of using non-degradable materials for tissue repair, degradable and absorbable biomaterials hold extensive capabilities to regenerate and reconstruct tissues such as bone, cartilage, muscle, skin, blood vessels, heart valves, nerves and many others [16-18]. These biomaterials are frequently used as scaffold materials for applications in tissue engineering and regenerative medicine [19]. In this strategy, specific cells are intended to grow into 3dimensional (3D) biomaterials-based porous scaffolds. After a while, native-like tissues or 
organs are formed which are used to replace and repair lost and failing tissues of patients. These engineered living tissues or organs could significantly reduce the replacement organ demand and offer new opportunities for therapy to accelerate the recovery of patients. Tissue engineering scaffolds contain a microenvironment which determines cell adhesion, growth, proliferation, differentiation and function. An ideal microenvironment supports cell growth and tissue formation, exchange of nutrients and waste products, and consists of extracellular matrix (ECM), growth factors, space for cell expansion, and external stimulation factors [20-23]. Scaffold materials are used as ECM and should be biocompatible, cell adhesive, biodegradable, and have proper mechanical properties. In addition, the materials may conduct electricity, release oxygen, and have magnetic or antibacterial properties. Scaffold structural parameters including pore size, porosity, and (patient-specific) 3D structure are important as well. Thus, scaffolds prepared from biocompatible and bioactive materials with an adequate 3D structure are essential for the formation of functional tissue [24-27].

In the early years, porous scaffolds were mainly prepared by solvent casting followed by particle leaching. As a result of technological developments, new techniques were applied in tissue engineering such as electrospinning, additive manufacturing, microfabrication and bioprinting [28][29]. These techniques enable to prepare porous scaffolds with complex structures. Currently, processing of biomaterials to match the advanced manufacturing techniques is one of the most researched areas. In conclusion, bioactive scaffolds, implants, grafts and other biomedical devices with specific structures can be fabricated by modern processing and manufacturing technologies for the urgent demand of a variety of tissues and organs.

\subsection{Materials}

Biomaterials can be divided into natural and synthetic polymers, metals and metal alloys, inorganic ceramics (natural and synthetic), carbon-based materials (graphite, graphene, carbon nanotube, carbon fiber, etc.), and composite materials [30-36]. The choice of materials for specific tissue repair is depending on the tissue characteristics and material properties. 
Table 1. Advantages and disadvantages of different classes of materials for tissue engineering and regenerative medicine.

\section{Advantages}

Disadvantages

\begin{tabular}{|c|c|c|}
\hline Polymers & $\begin{array}{l}\text { Biocompatible, biodegradable, } \\
\text { bioresorbable, low toxicity, } \\
\text { mechanical properties for both } \\
\text { soft and hard tissue engineering. }\end{array}$ & Lack of bioactivity. \\
\hline Metals & $\begin{array}{l}\text { Tough, high load bearing, good } \\
\text { mechanical properties. }\end{array}$ & $\begin{array}{c}\text { Too stiff causing tissue } \\
\text { degeneration, corrosion, toxic } \\
\text { ion release. }\end{array}$ \\
\hline Ceramics & $\begin{array}{l}\text { Bioactive such as osteo- } \\
\text { conductive and osteo-inductive. }\end{array}$ & Brittleness, fragmentation. \\
\hline Carbon-based materials & Conductive, low weight. & $\begin{array}{c}\text { Not able to be degraded and } \\
\text { resorbed. }\end{array}$ \\
\hline Composite materials & $\begin{array}{l}\text { Combination of advantages of } \\
\text { the separate components. }\end{array}$ & $\begin{array}{l}\text { Processing in such a way that the } \\
\text { advantages are properly } \\
\text { expressed. }\end{array}$ \\
\hline
\end{tabular}

Natural and synthetic polymers have been extensively used in tissue engineering and regenerative medicine because of their diverse properties, such as bioactivity, degradability, mechanical properties, processing ability. Natural polymers like silk fibroin, collagen, and gelatin are similar to the native ECM and generally show better cell adhesion and biocompatibility than synthetic polymers [37]. Even though scaffolds based on natural polymers hardly show chronic inflammation, toxicity or immunological reactions, which are frequently noticed in synthetic polymer scaffolds, poor mechanical properties are a drawback. On the other hand, synthetic polymers relatively easily allow tailoring of their molecular weight, degradation time, and mechanical properties for both hard and soft tissue engineering applications [38]. Biodegradable polymers such as poly(lactide) (PLA), poly(glycolide) (PGA), poly(lactide-co-glycolide) (PLGA), poly( $\varepsilon$-caprolactone) (PCL), poly(trimethylene carbonate) (PTMC) and their copolymers or blends are frequently used as scaffold materials in tissue engineering and regenerative medicine [39]. However, the utility of PLA and PGA as scaffold materials for implantation has been hampered by their acidic degradation products. Studies have shown a decrease in local $\mathrm{pH}$ caused by the acidic degradation products, which are harmful to cells, drugs, or proteins and can lead to tissue inflammation at the polymer/tissue 
interface [40][41]. Thus, scaffolds based on PLA, PGA, and their copolymers have sub-optimal degradation properties. In particular, PTMC has been suggested as an ideal polymer for biomedical applications [42][43]. As an amorphous polymer, PTMC has a low glass transition temperature ( $\mathrm{Tg}$ ) of $-17^{\circ} \mathrm{C}$ [44] [45]. The degradation products of PTMC are 1,3-propandiol and carbon dioxide. [46]. In contrast to the degradation of PLA and PGA, no acidic compounds are formed upon the degradation of PTMC [47][48]. Although PCL also does not form acidic degradation products, it degrades in bulk like PLA and PGA, which leads to abrupt scaffold fragmentation during tissue regeneration. PTMC degrades by surface erosion, resulting in prolonged mechanical strength during degradation which is beneficial for tissue engineering applications [49]. PTMC degradation rate can be tuned by varying its molecular weight and by adjusting the crosslink density of networks [47][50]. PTMC-based polymer networks have been extensively studied and applied in various biomedical applications [51].

Up to now, plenty of biomedical products based on synthetic polymers have been produced such as nerve guide conduits, vascular grafts, and artificial skin. Although these polymers are biocompatible and have adjustable degradation rates and mechanical properties, a drawback is they do not present bioactivity. For the engineering of complex tissues like bone, the use of only polymers does not meet the requirements for a suitable microenvironment to form a functional tissue. This can be solved by using a polymer-based composite, see below.

Metals and metal alloys with designed load-bearing mechanical properties are being used for joint replacement (hip and knee) and dental implants. They are biocompatible and nearly all are non-biodegradable. Most implants made from metals and metal alloys are intended to have a long implantation time, which may lead to corrosion and metal ion toxicity [32][52]. Besides that, metallic implants are much stiffer than host bone, which may lead to stress shielding resulting in bone resorption [53]. It should be noticed that metal-based micro- and nanoparticles ( $\mathrm{Au}$ and $\mathrm{Ag}$ ) display excellent conductivity, antimicrobial properties and other bioactive functions in medical applications [54].

Bio-glass is the first used bioactive inorganic ceramic able to induce bone formation and has been commercialized [55]. As components of native bone, calcium phosphate-based ceramics such as hydroxyapatite (HA), tricalcium phosphate (TCP) and biphasic calcium phosphate (BCP) are frequently used for bone tissue engineering as well [56]. Even though these ceramic materials resemble the natural inorganic component of bone and possess osteo-conductive and 
osteo-inductive properties, they are brittle and do not match the mechanical properties of bone [57].

Carbon-based biomaterials have been researched for decades and attract very much attention in biomedical applications due to excellent conductivity, unique structure and mechanical properties [58]. Graphene (single or multilayers) and carbon nanotubes (cylindrical carbon structure) are the mostly used carbon materials in neuronal, cardiac and bone tissue engineering. Moreover, they have also been used as secondary structural reinforcements to enhance the mechanical properties of tissue engineering scaffolds [59]. Graphene and carbon nanotubes express bioactivity in multiple processes like neurite outgrowth and extension, stem cell differentiation, osteogenic differentiation, and display antibacterial activity [60]. However, carbon materials are not able to be resorbed when applied in vivo.

The term "composite material" refers to the combination, on a macroscopic scale, of two or more materials, that differ in composition or morphology, in order to obtain specific chemical, physical and mechanical properties [61][62]. The advantage of using composite materials for biomedical applications is that a composite material may possess a combination of the best properties of the constituents. Composite materials offer useful properties such as bioactivity, electrical conductivity, oxygen supply and magnetic and antimicrobial properties. Polymerbased composites are composed of a polymer matrix and one or more fillers which provide physical, chemical or biological properties. As mentioned before, synthetic polymers generally lack bioactivity compared to bioactive ceramics, metal nano-particles, and carbon-based materials. A polymer/ceramic composite for bone grafting may present improved mechanical properties compared to either the neat polymer or the ceramic. This means that reinforced scaffolds with enhanced bioactivity and controlled resorption rates can be obtained by combining suitable polymers and ceramics [63]. A different application of composites in biomedical engineering is the use of composites with leachable components for the preparation of porous scaffolds. Leachable components include salt, sugar and crystallized solvent particles [64].

In this review, we summarize the properties of bioactive and non-bioactive polymer-based composites and their processing into structures for biomedical applications. Bioactivity of the composites includes osteo-conduction and -induction, electrical conductivity, magnetization, oxygenation, and antibacterial properties. Non-bioactive components of the composites include 
reinforcing and leachable fillers. Manufacturing techniques for the preparation of advanced structures using polymer-based composites are discussed as well.

\section{Fillers and particles incorporated in polymer matrices for biomedical applications}

Polymer-based composites can be divided into two main types regarding the filler incorporated in the polymer matrix. The first type are bioactive polymer-based composites, which contain bioactive fillers or particles. The second type are non-bioactive polymer-based composites, which contain e.g. reinforcing fillers or porogens (sacrificial particles added during processing for preparation of porous structures).

\subsection{Polymer-based bioactive composites}

\subsubsection{Polymer/bioactive ceramic composites}

Ceramics such as bioglass and bioactive calcium phosphate-based ceramics are synthetic bone graft materials widely used in bone tissue engineering [65]. Bioglass, invented by Hench and co-workers, was the first synthetic material found to interact with bone [66]. This degradable glass is composed of $\mathrm{Na}_{2} \mathrm{O}, \mathrm{CaO}, \mathrm{SiO}_{2}$ and $\mathrm{P}_{2} \mathrm{O}_{5}$. When used for bone regeneration in animal models, it was found that bioglass-based implants could not be removed without breaking the bone. This discovery inspired research on bioactive inorganic materials, resulting in the development of other types of bioglass and calcium phosphate ceramics. Bone is a natural composite consisting of inorganic ceramics such as calcium phosphate $(\mathrm{CaP})$, calcium sulfate $\left(\mathrm{CaSO}_{4}\right)$ and calcium carbonate $\left(\mathrm{CaCO}_{3}\right)$, and the polymer matrix collagen [67]. The collagen matrix facilitates cellular interactions and tissue formation, while the inorganic materials provide mechanical strength and support the regeneration of bone. The inorganic phase releases calcium ions which benefit bone cell proliferation and differentiation.

$\mathrm{CaP}, \mathrm{CaSO}_{4}$, and $\mathrm{CaCO}_{3}$ have been used separately or as composite fillers in a polymer matrix to fabricate substrates and scaffolds for bone regeneration [68]. $\mathrm{CaP}$ as a main component of native bone are able to be absorbed by bone cells in vivo. CaP can be divided into the following types: hydroxyapatite (HA), $\beta$-tricalcium phosphate $(\beta$-TCP), biphasic calcium phosphate $(\mathrm{BCP})$ and amorphous calcium phosphate $(\mathrm{ACP})$. CaP ceramics show bone forming activity 
(osteoconductivity or osteoinductivity), determined by their physical/chemical characteristics and surface structures [69]. Bone forming activity of CaP has been mostly shown in non-load bearing clinical situations involving orthopedic, dental, ear, nose and throat surgeries using compressed ceramic particles. CaP particles are inherently brittle and difficult to process, and not able to withstand load bearing conditions. Although synthetic polymers are generally easy to be processed into structures with tunable mechanical properties, they do not show satisfactory bone forming capacity. By combining the polymers and $\mathrm{CaP}$ particles, the resulting polymer-based ceramic composites can be used to fabricate bone grafts with suitable mechanical properties and bone forming activity [70].

Likewise, polymer-based bioglass ceramic composites were developed [71]. Initially, micronsize bioactive glass particles were used, which was followed by application of nano-size bioglass particles and fibres as fillers in polymer-based composites. Hong et al. investigated $\mathrm{PLA} /$ nano bioactive glass composite scaffolds for bone regeneration. In vitro studies showed that bioglass containing lower phosphorous and higher silicon content had a better bioactivity than bioglass with lower silicon and higher phosphorous content [72]. In addition, the mechanical properties of these composite scaffolds improved by loading of the nano-size bioactive glass fillers [73].

Nano-HA has been frequently used as filler in polymer-based scaffolds for bone formation. Both synthetic polymers like PLA, PCL, PGA, PLGA, PTMC, and natural polymers such as gelatin and collagen have been used [74,75]. The presence of HA in the polymer matrix is important as it affects protein adsorption and bone cell adhesion. Nano-HA fillers with a high aspect ratio enhance the mechanical properties of polymer-based bone grafts. PTMC scaffolds containing $40 \mathrm{wt} \%$ HA nanoparticles showed more bone formation than PTMC scaffolds with $20 \mathrm{wt} \%$ HA upon implantation in calvarial defects in rabbits [76]. Moreover, this study indicated that surface enrichment of the HA nanoparticles in the PTMC-based ceramic composites was a key factor in the osteogenic potential of the bone grafts.

PCL/ $\beta$-TCP composite scaffolds were manufactured by Park et al. using 3D printing [77]. Highly porous scaffolds were obtained with a high $\beta$-TCP content. In vitro studies showed that the scaffolds effectively promoted cell growth and osteogenic differentiation of mouse mesenchymal stem cells. Konopnicki et al. also 3D-printed PCL/ $\beta$-TCP composite scaffolds for bone regeneration, which were seeded with porcine bone marrow progenitor cells and 
implanted into porcine mandibular defects. The results showed good bone penetration depth with angiogenesis in the center of the constructs [78].

PCL/BCP composite scaffolds for bone regeneration were prepared as well. By means of a solvent casting and salt leaching method, porous PCL/BCP scaffolds, with $200 \sim 500 \mu \mathrm{m}$ pore size, were obtained. After 7 days culturing of human mesenchymal stem cells, the PCL/BCP composite scaffolds showed 4 times higher alkaline phosphatase activity than control PCL scaffolds [79]. Peroglio et al. used PCL to infiltrate brittle BCP scaffolds for bone engineering. The composite scaffolds were cytocompatible with human bone marrow stromal cells. Moreover, the mechanical properties of the scaffolds significantly improved by incorporation of PCL [80].

ACP nanoparticles also showed biocompatibility and bioactivity. PDLLA/ACP composite nanofibers were prepared and evaluated in vitro by Ma et al. Surface roughness of the composite nanofibers significantly increased with increasing content of ACP. Biomineralization of the composite nanofibers was found after 1 day in simulated body fluid, and further increased after 7 days. Osteoblast-like MG63 cells were seeded on PDLLA/ACP composite nanofiber scaffolds, and good cell adhesion and cell spreading behavior were obtained [81].

$\mathrm{CaSO}_{4}$ and $\mathrm{CaCO}_{3}$ are also considered as fillers to form polymer-based composites for bone regeneration [82] [83]. Silica $\left(\mathrm{SiO}_{2}\right)$ nanoparticles are investigated as well [84][85].

\subsubsection{Polymer/electrically conductive filler composites}

Electrically conductive materials have received increasing attention of academic and industrial researchers to explore potential biomedical applications, e.g. in the fields of biosensing, targeted drug delivery, and tissue engineering [86][87]. These materials could stimulate cell adhesion, proliferation, differentiation, migration, function, and further drive cell activities and tissue formation with or without exogenous electrical stimulation [88] [89]. Native tissues such as nerve, muscle, lung, cardiac and skeletal muscle, have conductivity values between 0.03 and $0.6 \mathrm{~S} / \mathrm{m}$ [90][91]. Therefore, tissue engineering scaffolds fabricated from electrically conductive materials are believed to accelerate tissue formation and regeneration [92]. 


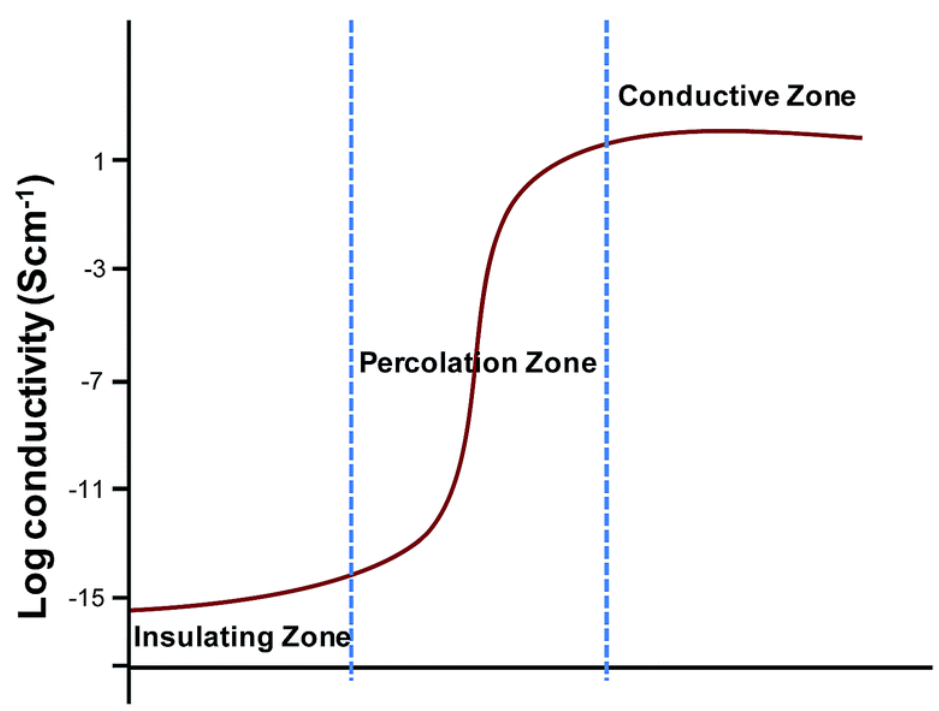

Filler Concentration (\%)

Figure 1. Percolation curve of electrically conductive filler in polymer matrix. [96]

Conductive polymers and composites of polymers and conductive fillers have been used as materials to fabricate electrically conductive scaffolds [93][94]. Conducting polymers such as polypyrrole (PPY), polyaniline (PAN), polythiophene (PTH) and poly (3,4-ethylene dioxythiophene) (PEDOT) were prepared for neuronal tissue engineering. However, for in vivo application, conductive polymers are not ideal due to poor suturability, brittleness, and longterm toxicity [95]. An alternative strategy is to use biocompatible and biodegradable polymers combined with conductive fillers. These are mainly divided in two classes: carbon-based nanofillers (carbon nanotubes, graphene derivatives) and metal particles (gold and silver particles). These fillers show high electrical conductivity and biocompatibility, and conductive polymer-based composites containing these fillers have been widely used for peripheral nerve regeneration and cardiac tissue engineering [96].

The conductivity of polymer/conductive filler composites is dependent on the formation of conductive paths of fillers distributed in the polymer matrix [97]. The formation of conductive paths is affected by the amount of fillers in the polymer matrix as well as the geometry and intrinsic properties of the fillers. In addition, interactions between filler and matrix are important for a homogenous distribution of the filler in the matrix. As shown in Figure 1, the conductivity of a polymer-based composite initially shows a small increase with increasing amount of conductive filler. Upon reaching the percolation threshold, the conductivity dramatically increases and finally reaches a maximum [96][98]. When the amount of 
conductive filler is above the percolation threshold, a continuous conductive network is formed throughout the composite. A high aspect ratio (ratio of length to diameter) of conductive fillers was found to contribute to the conductivity of polymer-based composites [98][99].

Carbon nanotubes (CNTs) are hollow nanostructures consisting of carbon atoms with excellent mechanical properties and high electrical conductivity. Crowder et al. prepared electrically conductive PCL/CNT composite scaffolds by electrospinning for cardiac tissue engineering. The highest conductivity $(0.035 \mathrm{~S} / \mathrm{cm})$ of this fibrous scaffold was obtained with $3 \mathrm{wt} \%$ incorporated CNTs. Differentiation of human mesenchymal stem cells in these scaffolds was found to be dependent on the substrate conductivity under DC electrical stimulation [99]. Other PCL/CNT composite scaffolds were prepared for nerve regeneration by Zhou et al. They showed improved PC-12 cell growth and differentiation in the conductive PCL/CNT composite scaffolds compared to neat PCL scaffolds. In addition, both proliferation and neuronal cell extension benefited from electrical stimulation, indicating the potential for application in nerve regeneration [100].

Single and multilayer graphene are known as high aspect ratio two dimensional nanosheets with ultra-high electrical conductivity and excellent mechanical properties. The common forms of graphene derivatives are graphene oxide (GO) and reduced graphene oxide (rGO). rGO is usually obtained by thermal or chemical reduction of GO, and has a higher conductivity than GO. rGO is more hydrophobic than GO and easily forms non-reversible aggregates because of van der Waals forces and $\pi-\pi$ stacking interactions. Therefore, both small molecules and polymers were used to modify the surface of rGO particles in order to obtain homogenous dispersions in a polymer matrix. Recently, polymer-based composites with rGO as conductive filler have drawn a lot of attention for biomedical applications. Sayyar et al. prepared PCL/rGO composite materials, both by solvent mixing and covalent linking of PCL to rGO. The latter method resulted in higher conductivity with lower amount of rGO than solvent mixing. In addition, well-dispersed rGO in the polymer matrix improved the mechanical properties of the $\mathrm{PCL} / \mathrm{rGO}$ composites [101]. Shin et al. also used covalent linking to prepare gelatin/rGO composite scaffolds for cardiac tissue engineering. The electrical conductivity and mechanical properties of the scaffolds were significantly improved by incorporation of rGO filler. Excellent cardiomyocyte viability, proliferation, and maturation were observed on the gelatin/rGO composite hydrogels. Moreover, the cells showed stronger contractility and faster spontaneous beating on the gelatin/rGO composite hydrogels compared to gelatin hydrogels [102]. 
Gold nanoparticles are regarded as ideal materials for nanomedicine, and widely used in imaging, theranostics and controlled drug delivery owing to facile synthesis, modification, tunable structure (spheres, rods, nanoplate, etc.), physicochemical properties, and biocompatibility [103][104]. Gold nanoparticles have a high electrical conductivity and have been incorporated in polymer matrices for biomedical applications. Navaei et al. prepared gelatin/gold nanorod composite substrates by solvent mixing and photo-crosslinking for cardiac regeneration. Both electrical conductivity and mechanical properties were improved by incorporation of the gold nanorods. Good retention, spreading and distribution of cardiac cells were observed on the conductive gelatin/gold nanorod composite hydrogel. Notably, cell-cell coupling and robust synchronized (tissue-level) beating behavior were observed [105]. PCLgelatin/gold nanoparticle composite fibrous scaffolds were also reported, showing that the assembly of a functional cardiac tissue was improved on the conductive surface [106].

Electrically conductive polymer-based composites offer opportunities to apply electrical stimulation to cells and tissues. Electrical stimulation could direct, concentrate and isolate cell responses. Furthermore, cells could grow aligned and tissue may orientate by using electrical stimulation. Encouraging results based on electrically conductive polymer-based composites were obtained with or without electrical stimulation in cardiac tissue engineering, wound healing and nerve regeneration. However, drawbacks of long-term toxicity and nondegradability of conductive fillers still remain as problems for future applications.

\subsubsection{Polymer/magnetic particle composites}

Magnetic nanoparticles (MNPs) have been regarded as one of the most attractive and important nanomaterials for biomedical applications in the fields of hyperthermia, magnetic resonance imaging, tissue engineering, targeted drug and gene delivery, biosensors and labs on chip owing to their chemical and physical properties [107][108]. Both metal (e.g. Fe, Ni, Co) and metal oxide $\left(\mathrm{Fe}_{3} \mathrm{O}_{4}, \mathrm{Fe}_{2} \mathrm{O}_{3}\right)$ particles were considered as magnetic fillers. However, pure metal particles are extremely sensitive to oxidation in conditions of high magnetization [109]. Therefore, iron oxide particles with a low sensitivity to oxidation and relatively strong magnetic response have been applied in vitro and in vivo for many years. $\mathrm{Fe}_{3} \mathrm{O}_{4}$ and $\mathrm{Fe}_{2} \mathrm{O}_{3}$ particles are biocompatible and relatively easy to be synthesized and functionalized [110].

MNPs and application of magnetic forces have been introduced in targeted drug delivery and tissue engineering research. Drugs or bioactive compounds can be loaded on MNPs. Using an 
external magnetic field, the particles can be directed to the target area. Likewise, cells loaded with magnetic particles can be adhered on a substrate or scaffold using an external magnetic field. Magnetizable scaffolds have been developed as well. When a scaffold is magnetic, an external magnetic field generates much higher magnetic field gradients as compared to a nonmagnetic scaffold. Moreover, when superparamagnetic iron oxide particles are used (size $<20$ $\mathrm{nm}$ ), the magnetism of the scaffold can simply be turned off by removing the external magnetic field. Such a magnetizable scaffold could be used to attract MNPs loaded with bioactive compounds multiple times to the scaffold after implantation, with varying payloads in time [111]. Moreover, by using an alternating external magnetic field, the temperature of the scaffold increases which could be applied for drug release or cancer treatment [112].

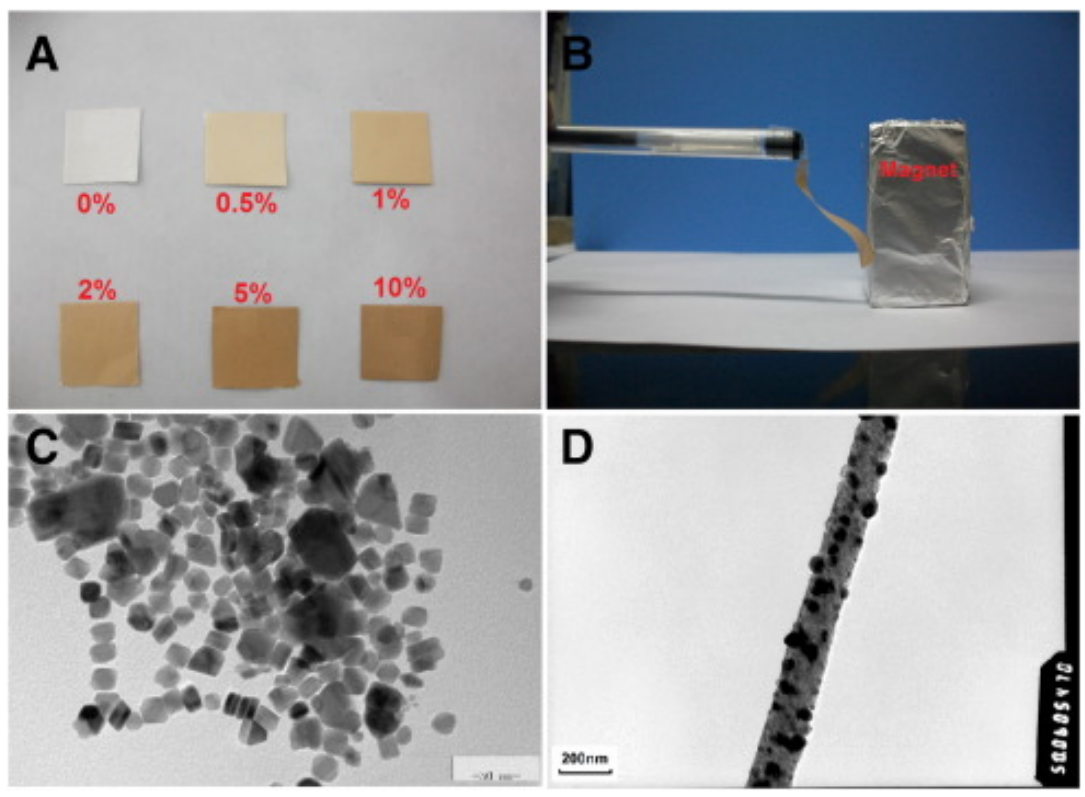

Figure 2. Photograph of $\mathrm{PCEC} / \mathrm{Fe}_{3} \mathrm{O}_{4}$ fibrous membranes with increasing $\mathrm{Fe}_{3} \mathrm{O}_{4}$ content (A), magnetic response of a $\mathrm{PCEC} / \mathrm{Fe}_{3} \mathrm{O}_{4}$ composite membrane with $10 \mathrm{wt} \% \mathrm{Fe}_{3} \mathrm{O}_{4}$ particles (B), TEM images of the $\mathrm{Fe}_{3} \mathrm{O}_{4}$ particles $(\mathrm{C})$ and the $\mathrm{PCEC} / \mathrm{Fe}_{3} \mathrm{O} 4$ fiber containing $10 \mathrm{wt} \% \mathrm{Fe}_{3} \mathrm{O}_{4}$ particles (D). [113]

Zhang et al. prepared novel fibrous polymer/magnetic particle composite scaffolds by electrospinning, composed of the tri-block copolymer poly(e-caprolactone)-poly(ethylene glycol)-poly( $\varepsilon$-caprolactone) (PCL-PEG-PCL or PCEC) and $\mathrm{Fe}_{3} \mathrm{O}_{4}$ nanoparticles with an average size of $18 \mathrm{~nm}$. Without an external magnetic field, $\mathrm{PCEC} / \mathrm{Fe}_{3} \mathrm{O}_{4}$ scaffolds with 2-10 $\mathrm{wt} \%$ nanoparticles showed improved NIH $3 \mathrm{~T} 3$ cell adhesion and proliferation as compared to 
neat PCEC scaffolds. Moreover, the $\mathrm{PCEC} / \mathrm{Fe}_{3} \mathrm{O}_{4}$ composites showed low cytotoxicity and hold great potential for skin tissue engineering [113].

Cai et al. prepared PLLA/superparamagnetic $\mathrm{Fe}_{3} \mathrm{O}_{4}$ nanoparticle composite scaffolds by electrospinning. Compared to PLLA scaffolds, composite scaffolds with 2.5-5.0 $\mathrm{wt} \% \mathrm{NPs}$ stimulated the proliferation and differentiation of MC3T3-E1 osteoblasts, which further increased by application of a $100 \mathrm{mT}$ external magnetic field [114].

Panseri et al. implanted collagen- $\mathrm{HA} / \mathrm{Fe}_{3} \mathrm{O}_{4}$ nanoparticle composite scaffolds in bone defects in rabbits. The NP content was $7 \mathrm{wt} \%$ and the particles had a size $<50 \mathrm{~nm}$. In some cases, a $\mathrm{NdFeB}$ magnet was co-implanted in contact with the scaffold, generating a $1.2 \mathrm{~T}$ magnetic field. With and without magnetic field, the extent of bone formation in the scaffolds was the same. With magnetic field, however, both the scaffold and newly formed bone were oriented, which may shorten remodeling and more quickly generate mature bone [115].

The above concepts could also be applied for magnetic scaffold fixation after implantation, or for development of scaffolds with intrinsic ability to stretch or move. Furthermore, magnetically responsive surfaces and hydrogels are currently being investigated [116].

\subsubsection{Polymer/oxygen-generating particle composites}

Oxygen is one of the most important factors for tissue growth and survival. Even though a promising tissue engineering scaffold with porous channels could provide nutrients and oxygen by diffusion, in the early stages after implantation of a construct the lack of vasculature to support an adequate supply of nutrients and oxygen limits tissue survival which is a challenge for clinical translation [117]. For example, during implantation of a tissue-engineered construct for a large bone defect, the in situ vasculature is damaged and disrupted. The implanted cells on the scaffold will suffer anoxia and nutrient deficiency in the concurrent wound bed before generation of new vasculature. Methods to vascularize engineered tissue and to connect the vasculature to the host circulation are still in development and not able to solve the oxygen demand of implanted constructs. An emerging approach is the use of oxygen-generating scaffolds to provide the initial oxygen supply. Calcium peroxide, magnesium peroxide, and sodium percarbonate are very suitable as oxygen-generating materials and have been successfully applied in tissue engineering [118]. The mechanism of oxygen generation is based 
on formation of hydrogen peroxide upon hydrolysis or dissolution in water, which will subsequently release oxygen. The reaction equations are as follows:

$\mathrm{CaO}_{2}$ (calcium peroxide) $+2 \mathrm{H}_{2} \mathrm{O} \rightarrow \mathrm{Ca}(\mathrm{OH})_{2}+\mathrm{H}_{2} \mathrm{O}_{2}$

$\mathrm{MgO}_{2}$ (magnesium peroxide) $+2 \mathrm{H}_{2} \mathrm{O} \rightarrow \mathrm{Mg}(\mathrm{OH})_{2}+\mathrm{H}_{2} \mathrm{O}_{2}$

$\left(\mathrm{Na}_{2} \mathrm{CO}_{3}\right)_{2} \cdot 3 \mathrm{H}_{2} \mathrm{O}_{2}$ (sodium percarbonate) $\rightarrow 4 \mathrm{Na}^{+}+2 \mathrm{CO}_{3}{ }^{2-}+3 \mathrm{H}_{2} \mathrm{O}_{2}$

$2 \mathrm{H}_{2} \mathrm{O}_{2} \rightarrow \mathrm{O}_{2}+2 \mathrm{H}_{2} \mathrm{O}$

Polymer/oxygen-generating particle composites have been used to prepare 'breathing scaffolds' for the engineering of bone, cardiac, muscle and skin tissue. The polymer matrix properties affect the oxygen-release kinetics, which influence cell viability, proliferation and differentiation. Hydrophobic polymers can prevent water absorption and slow down the rate of oxygen release, while hydrophilic polymers may increase the oxygen release rate by rapid diffusion of water into the polymer structure, thus decreasing the sustainability of oxygen release. The solubility of the oxygen-generating compounds in water affect the oxygen release as well. $\mathrm{CaO}_{2}$ has a higher solubility in water than $\mathrm{MgO}_{2}$ (calcium peroxide $1.65 \mathrm{~g} / \mathrm{L}$ and magnesium peroxide $0.86 \mathrm{~g} / \mathrm{L}$ at room temperature) [119]. Therefore, $\mathrm{CaO}_{2}$ has been frequently investigated as oxygen-generating compound in polymer-based composites for tissue engineering.

A polydimethylsiloxane (PDMS) $/ \mathrm{CaO}_{2}$ composite was prepared by Pedraza et al. [120]. Although they showed an effectively slow release of oxygen, PDMS is a non-degradable polymer which is not ideal as scaffold material for tissue engineering. Steg et al. prepared $\mathrm{PLA} / \mathrm{CaO}_{2}$ and $\mathrm{PLGA} / \mathrm{CaO}_{2}$ composites for the controlled release of oxygen [121]. However, oxygen release from both composites was faster than from control $\mathrm{CaO}_{2}$ particles. This was explained by a relatively low $\mathrm{pH}$ induced by hydrolysis of the lactide-based polymers, leading to a higher solubility of the intermediate $\mathrm{Ca}(\mathrm{OH})_{2}$, which accelerated the reaction towards $\mathrm{H}_{2} \mathrm{O}_{2}$ formation. Therefore, they prepared PTMC/CaO 2 composite microspheres which were able to release oxygen for a few weeks and promote mesenchymal stromal cell proliferation under hypoxic conditions in vitro [122]. Harrison et al. prepared PLGA/sodium percarbonate composite films for wound healing. The composite films released oxygen for a period of approximately $70 \mathrm{~h}$. Subcutaneous implantation of the composite films in a mouse model for ischemic skin, showed significantly lower skin necrosis and higher skin viability after 7 days compared to implantation of control PLGA films [123]. 
Sustainable oxygen-releasing scaffolds were prepared from a $\mathrm{PU} / \mathrm{CaO}_{2}$ composite by Siekh et al. The scaffolds released oxygen over a period of 10 days and in vitro cardiomyoblast cell viability on the composite scaffolds under hypoxic conditions was better than on control PU scaffolds. Subcutaneous implantation of the composite scaffolds in a mouse model for ischemic skin, showed that skin necrosis could be prevented up to 9 days [124].

Oxygen deficiency after myocardial infarction leads to massive cardiac cell death. Reintroduction of oxygen into the infarcted area may protect cardiac cells and promote cardiac repair. Fan et al. prepared core-shell microspheres based on PLGA covered with a PVP/ $/ \mathrm{H}_{2} \mathrm{O}_{2}$ complex. The microspheres released oxygen for 28 days and supported cardiac cell survival under hypoxic conditions in vitro [125].

\subsubsection{Polymer/antibacterial particle composites}

Bacterial infection of biomedical implants during surgical procedures remains challenging. Adhesion of bacteria on the implants may result in the formation of biofilms which are difficult to remove and may lead to failure of patient recovery [126-128]. Bacterial infection of the implant can occur simultaneously with implantation, or spread from the blood or a nearby infection site present in the patient [129]. Therefore, the availability of biomedical materials with antibacterial properties is of great importance. Various polymer-based composites with antibacterial particles have been developed [130]. Silver nanoparticles (AgNPs), magnesium oxide $(\mathrm{MgO})$, and zinc oxide $(\mathrm{ZnO})$ are frequently used and show activity against antibioticresistant bacteria [131-133].

There are two potential antibacterial mechanisms of AgNPs: direct contact of the particles with the bacterial cell membrane leading to leakage of cellular contents, and interaction of $\mathrm{Ag}^{+}$ions with cellular structures and biomolecules such as enzymes, lipids and DNA. For the development of biocompatible polymer/AgNP composites with antibacterial properties, the dose-dependent toxicity of Ag to tissue cells has to be taken into account. Fortunati et al. prepared PLGA/AgNP composite films, which started to show weight loss due to degradation of PLGA after 25 days incubation in PBS at $37^{\circ} \mathrm{C}$. This time point coincided with an increase of $\mathrm{Ag}^{+}$release from the films, probably due to increased water influx in the films promoting Ag oxidation [134]. PLCL/AgNP composite scaffolds were fabricated by electrospinning with $0.5 \mathrm{mg}$ or $1.0 \mathrm{mg} \mathrm{Ag}$ loading per g scaffold. The biocompatibility of the scaffolds was evaluated by culturing of human epidermal keratinocytes, and antibacterial properties were investigated 
with staphylococcus aureus and escherichia coli cultures. Although both scaffolds were able to inhibit bacterial cell growth, the scaffold with the higher Ag content was also toxic to the keratinocytes [135]. Madhavan et al. prepared electrospun PCL/AgNP composite scaffolds for vascular tissue engineering. Scaffolds with $0.1 \mathrm{wt} \%$ AgNPs showed antibacterial properties without toxicity to cultured endothelial cells [136].

Bakhsheshi-Rad et al. fabricated electrospun PCL/MgO-Ag composite nanofibers as coating on biodegradable $\mathrm{Mg}$ alloy implants. Nanofibers containing $1-3 \mathrm{wt} \% \mathrm{MgO}$ and $1 \mathrm{wt} \% \mathrm{Ag}$ showed efficient antibacterial behavior toward Escherichia coli and Staphylococcus aureus [137]. PLA/ZnO nanoparticle composite scaffolds were electrospun by Rodríguez-Tobías et al. Tensile strength, toughness and Young's modulus of the scaffolds increased by addition of $\mathrm{ZnO}$, and reached a maximum at $3 \mathrm{wt} \% \mathrm{ZnO}$. The scaffolds showed antibacterial properties when they contained more than $1 \mathrm{wt} \% \mathrm{ZnO}$ [138]. $\mathrm{MgO}$ and $\mathrm{ZnO}$ have been shown to generate reactive oxygen species, leading to lipid peroxidation and bacterial membrane leakage [139][140].

\subsection{Polymer-based non-bioactive composites}

\subsubsection{Polymer/reinforcing micro- and nano-fiber composites}

Mechanical properties of tissue engineering scaffolds are one of the most important parameters, especially in the case of load bearing applications. Inorganic, organic, and carbon fillers and fibers have been used as reinforcements to improve the mechanical properties of polymerbased scaffolds for biomedical applications. Bioactive fillers that improve mechanical properties of scaffolds were discussed in 2.1.1. Concerning non-bioactive fillers, polymer fibers are mostly being used to reinforce polymer-based scaffolds. Zhang et al. prepared tough biodegradable materials consisting of polymer-polymer composites. A PTMC matrix was reinforced by electrospun PLA fibers. Incorporation of a small amount ( $5 \mathrm{wt} \%$ ) of PLA fibers, resulted in an increase of Young's modulus and tensile strength [141].

\subsubsection{Polymer/porogen particle composites}

Porous scaffolds with a proper porosity, pore size, and pore shape are essential for successful tissue engineering. Current methods to fabricate porous scaffolds include particle leaching, thermally induced phase separation, gas foaming, electrospinning, and additive manufacturing. Among these methods, particle leaching is the easiest technique to obtain 3D scaffolds with 
varying pore size and porosity [142][143]. Sodium chloride, sugar and other leachable particles are being used. Sodium chloride is most popular as porogen, owing to its low cost, easy way of fractionation in different sizes, and the convenience of using water as leaching solution [144]. To prepare a porous scaffold, leachable particles are mixed in a polymer solution, thus forming a polymer/leachable particle composite. For a fully leachable scaffold, the particle content should be high enough to allow interconnection of the particles. By adjusting particle content and particle size, 3D scaffolds can be fabricated with varying porosity and pore size, which affect cell seeding in the scaffolds. Song et al. used sodium chloride particles as porogen to prepare PTMC scaffolds for vascular tissue engineering and showed that a pore size around $150 \mu \mathrm{m}$ was favorable for smooth muscle cell proliferation [145].

Pore interconnectivity is also important for tissue formation. Using a salt fusion method, the interconnectivity of pores in a scaffold can be improved [146]. Pore shape can be tuned by the shape of the particles. Liang et al. prepared quasi-spherical particles as porogen to fabricate porous PLGA scaffolds, by wobbling small sodium chloride particles in melted sucrose [147]. Compared to the use of cubic salt particles, using the quasi-spherical particles resulted in higher pore interconnectivity, less time for porogen leaching, and less residual porogen present in the scaffolds after leaching. Moreover, the latter scaffolds showed improved proliferation of mesenchymal stem cells, probably due to a rougher surface of the pores.

Tissue engineering scaffolds with designed porosities and pore structures can be fabricated by additive manufacturing. Among the various additive manufacturing techniques, stereolithography (SLA) is the most accurate and allows printing at the lowest resolution. Using currently available laser-based and digital light processing SLA machines, structures can be built at a resolution of 10-150 $\mu \mathrm{m}$ [148][149]. Although pores of these sizes suffice many tissue engineering applications, it can be advantageous if the scaffold contains (sub)micron pores in the struts of the polymer structure. This can be accomplished by printing a polymer/leachable particle composite. Mu et al. fabricated poly(ethylene glycol) scaffolds by digital light processing with rectangular macro-pores of $2 \mathrm{~mm}$ and micro-pores of $75-180 \mu \mathrm{m}$ by inclusion of sacrificial sodium chloride particles during printing [150]. 


\section{Polymer-based composite structures for biomedical applications}

\subsection{Porous scaffolds prepared by particle leaching}

Particle leaching is a very convenient method to prepare porous TE scaffolds. It has been used for decades due to its simplicity and low cost. A polymer solution is combined with uniformly distributed leachable particles of a certain size range, after which the mixture is cast on a mold or template. The solvent is either evaporated or extracted, yielding a polymer/leachable particle composite [151]. Subsequently, the polymer/leachable particle composite is immersed into a solvent for the particles, to obtain a porous scaffold with a specific structure. The amount of particles in the composite has to be higher than a threshold value, otherwise the particles cannot be completely leached from the polymer matrix. The main benefits of this method include the ability to adjust the porosity by tuning the amount of leachable particles, and to vary the pore size by selecting the size of the particles [152].
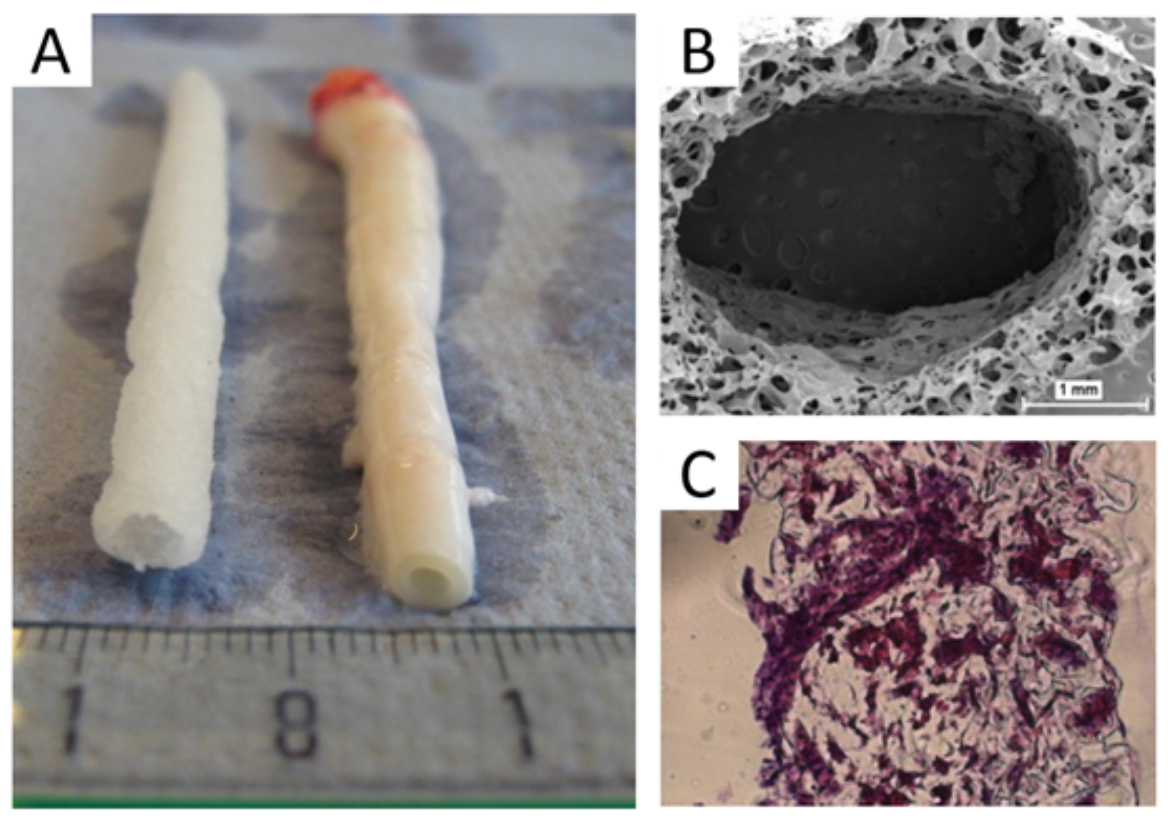

Figure 3. A, Photograph of a porous tubular PTMC scaffold (left) and a porcine carotid artery (right). B, SEM image of a cross-section of a tubular PTMC scaffold. C, SMCs cultured for 7 days in a porous tubular PTMC scaffold in a pulsatile flow bioreactor. Cross-section stained with hematoxylin and eosin, magnification 100x.

Song et al. developed a porous tubular PTMC scaffold for vascular TE by solvent casting of a mixture of PTMC and $\mathrm{NaCl}$ particles on a glass rod. After drying, the PTMC was crosslinked 
by gamma-irradiation and the particles were leached in water [153]. The interconnected porous scaffold had an average pore size of $110 \mu \mathrm{m}$, and a porosity of $85 \%$. Human smooth muscle cells (SMCs) were seeded into the pores of the tubular scaffolds and cultured for 14 days in a pulsatile flow bioreactor. The porous structure facilitated the proliferation of the SMCs. The radial tensile strength of the constructs increased from 0.16 to $0.47 \mathrm{MPa}$, due to the presence of SMCs and deposition of extracellular matrix in the structures. A tubular PTMC scaffold, its porous structure as well as histology after culturing with SMCs are shown in Figure 3.

Particle leaching can also be combined with other techniques such as $3 \mathrm{D}$ printing. This enables the fabrication of more complex 3D structures, as compared to solvent casting on a mold. By using a photo-crosslinkable polymer-based and particle-containing resin, a designed porous structure can be printed. The polymer struts contain the particles, which can subsequently be leached to obtain a dual porous structure. Mu et al. used this fabrication method to prepare 3D PEG structures, containing $2 \mathrm{~mm}$ rectangular macropores by printing and $75-180 \mu \mathrm{m}$ micropores by leaching of $\mathrm{NaCl}$ particles [150].

\subsection{Fibrous structures prepared by electrospinning}

Electrospinning (ES) has been widely used in TE to develop micro- or nano-fibrous scaffolds with a large surface area and high porosity. ES equipment is composed of a syringe pump, a metal needle, a high-voltage power supply, and a grounded collector. The latter can be of various shapes, such as a flat plate or a mandrel, depending on the desired shape of the scaffold. A polymer solution or melt is driven through the charged needle, resulting in a charged liquid jet that is attracted by the grounded collector. During its travel to the collector, the jet solidifies due to solvent evaporation or cooling. This results in the deposition of micro- or nano-fibers on the surface of the collector [154]. Electrospun fibrous mats based on biodegradable polymers such as PLA and PCL showed better cell adhesion than non-fibrous substrates of the same materials [155]. To combine the benefits of synthetic polymers and functional fillers, fibrous polymer-based composite scaffolds were prepared by ES of mixtures of polymers and fillers. Successful incorporation of functional fillers in electrospun polymer fibers is dependent on the size of the fillers. Graphene sheets, carbon nanotubes, nano-hydroxyapatite and silver particles of either nano- or sub-micron size are suitable fillers for polymer fibers to obtain bioactive scaffolds [156]. 


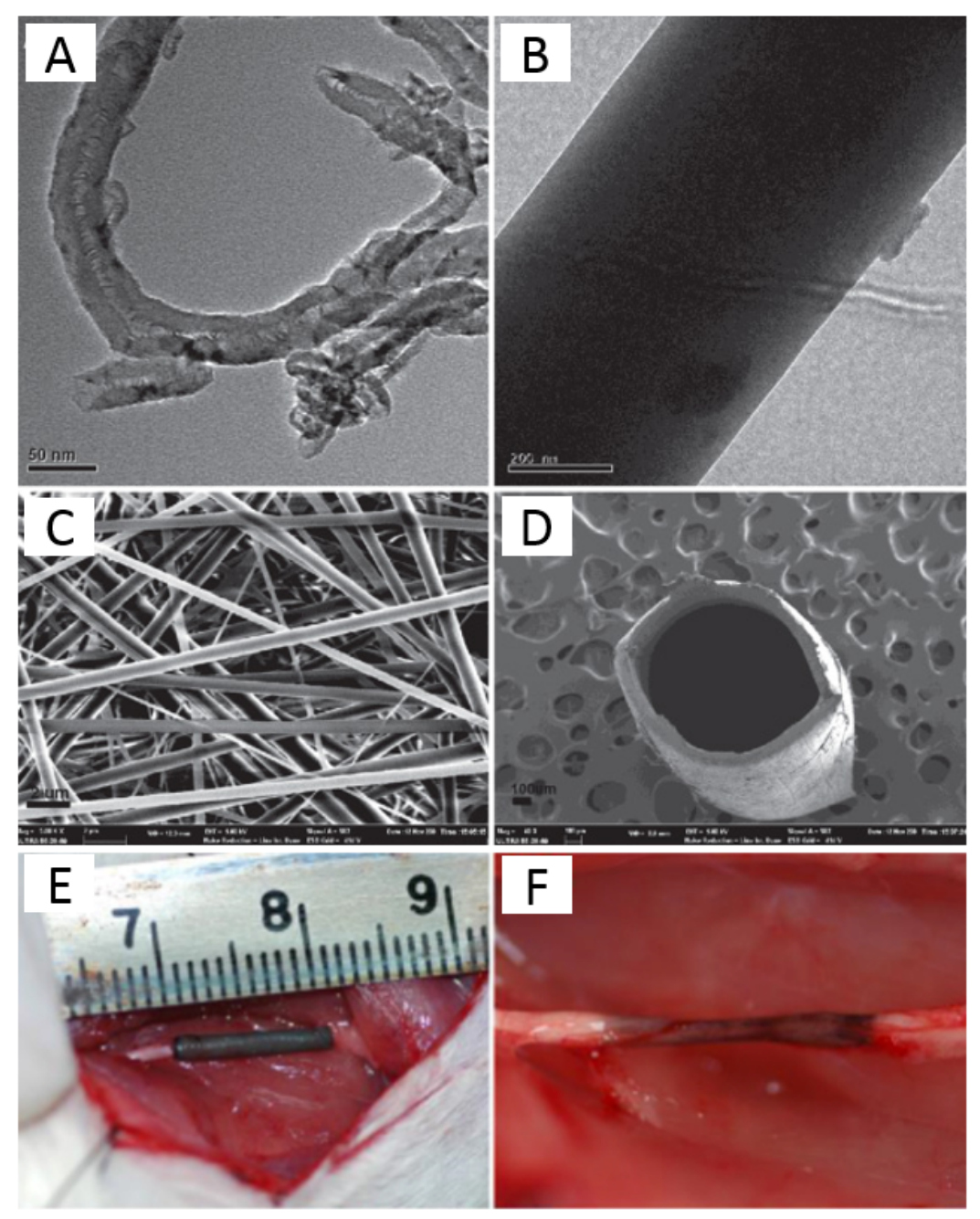

Figure 4. TEM images of carbon MWNTs (A) and electrospun PCL-collagen/carbon MWNT composite fiber (B); SEM images of PCL-collagen/carbon MWNT composite fiber mesh (C) and nerve guide conduit (D). Surgical implantation of PCL-collagen/carbon MWNT composite nerve guide conduit to bridge an $8 \mathrm{~mm}$ sciatic nerve defect in a rat $(E)$ and macroscopic image of the regenerated nerve four months postoperatively. The bar in $A=50 \mathrm{~nm}$, in $B=200 \mathrm{~nm}$, in $C=2 \mu \mathrm{m}$, in $D=100 \mu \mathrm{m}$. [157]

Yu et al. fabricated PCL-collagen/carbon multi-walled nanotube (MWNT) composite fibrous nerve guides by ES for peripheral nerve regeneration [157]. Figure 4 shows the carbon MWNTs, composite fibers, a conduit, and implantation of the conduit. Upon loading with carbon MWNTs, the hydrophilicity of the scaffolds increased due to carboxyl groups present in the MWNTs. The mechanical properties of PCL-collagen fibrous meshes, in terms of Young's modulus, elongation at break and maximum strength, increased by incorporation of carbon MWNT filler. The degradation rate of the composite scaffolds was lower than that of the PCLcollagen scaffolds. Schwann cell adhesion and elongation were enhanced on the electrospun 
composite scaffolds. In vivo studies demonstrated that PCL-collagen/carbon MWNT composite conduits effectively promoted sciatic nerve regeneration in rats and prevented muscle atrophy, without serious chronic inflammation.

PCL/GO composite fibrous scaffolds were prepared by Chaudhuri et al. by means of ES. The electrical conductivity of PCL scaffolds was enhanced by incorporation of GO particles. The composite scaffold exhibited excellent properties for myoblast differentiation, and has potential to be applied for skeletal muscle regeneration [158].

Even though ES is a relatively simple and quick method to manufacture fibrous scaffolds that support cell adhesion and proliferation, fabrication of complex structures with load bearing architectures is still a challenge and limits its biomedical applications [159].

\subsection{Patient-specific structures and implants prepared by additive manufacturing}

Additive manufacturing (AM), also known as 3D printing, offers an engineering route to build complex structures in a rapid and cost-effective way. A wide range of biomaterials such as polymers, metals, ceramics have been processed to become 3D printable for AM applications, according to the requirement and mechanism of the printing equipment [160]. AM techniques include laser-based stereolithography (SLA), digital light processing (DLP), fused deposition modeling (FDM), selective laser sintering (SLS), and extrusion-based 3D printing [161][162]. $\mathrm{AM}$ is widely used for biomedical applications, e.g. to fabricate tissue engineering scaffolds, bone implants, and microfluidic chips for organ models. In contrast to traditional fabrication approaches such as solvent casting and ES, AM involves layer by layer printing of virtual slices of a designed 3D structure. It is a rapid manufacturing method to obtain complex scaffolds and patient-specific implants [163]. Synthetic polymers such as PCL, PEG, PDLLA, PTMC and natural polymers such as gelatin and cellulose have been used to prepare scaffolds by AM for a variety of biomedical applications. Polymer-based composites with bioactive properties are interesting materials for the fabrication of functional structures by means of AM [164] [165].

Advantages of AM techniques to create biomimetic tissue structures include the formation of highly interconnected pores to facilitate tissue formation, and tuning of the mechanical strength by adjusting the design of the structure. Scaffolds built by AM can be based on a computer design or on imaging data, e.g. obtained by CT scanning. Among all AM techniques, laserbased SLA and DLP are most suitable for scaffold fabrication due to their high resolution, 
ability to build complex structures, and ease of tailoring the scaffold properties by adjusting the liquid resin formulation [166][167].

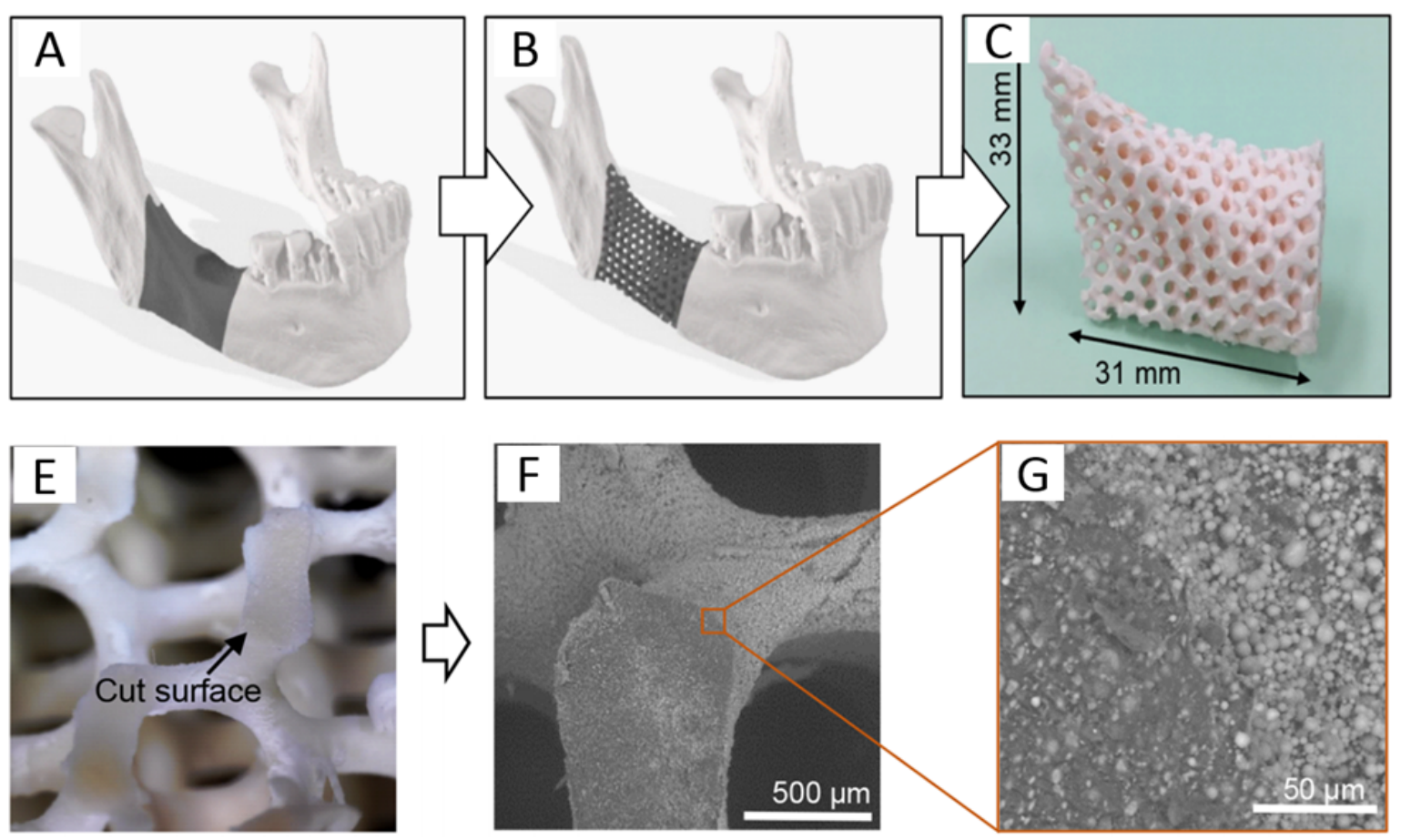

Figure 5. A, imaging data (grey) to fix a defect in a human jaw; B, defect model of porous patientspecific implant; C, PTMC/TCP composite implant printed by DLP. E, macroscopic image of a cut PTMC/TCP implant with gyroid pore architecture; F, SEM image of the cut surface of this scaffold; $G$, high magnification of this cut surface, where polymer matrix and TCP filler can be observed clearly. [168]

Dienel et al. fabricated bioresorbable implants based on a PTMC/TCP composite resin for repair of damaged bone tissue [168]. 3D composite scaffolds containing up to $60 \mathrm{wt} \% \beta$-TCP could be built by DLP. By using a slightly lower $\beta$-TCP content of $51 \mathrm{wt} \%$, a large-size patientspecific implant was manufactured at high resolution based on imaging data. The mechanical properties of the structure significantly increased by addition of $\beta$-TCP. The porous implant containing bioactive TCP and biodegradable PTMC perfectly matched the defect shape of the patient. Figure 5 shows the imaging-based defect model and the porous PTMC/ $\beta$-TCP composite implant.

Geven et al. used PTMC/HA composites to fabricate patient-specific orbital floor implants by DLP based on CT imaging data [169]. Photo-crosslinkable PTMC-based resins were 
formulated with $20 \mathrm{wt} \%$ and $40 \mathrm{wt} \%$ nano-HA. Mechanically stable orbital floor implants were precisely prepared using these composite resins, and shown to support the culturing of human bone marrow mesenchymal stem cells.

Although AM is able to improve the performance of biomaterial-based structures for medical applications, there are still challenges. Optimal scaffold structures for specific biomaterials are not well known, as relevant mechanical, physical and biological properties of the biomaterials should be considered during the design phase. The optimal shape, pore size and structure for specific applications still need to be determined. There are limited biomaterials available for processing by AM techniques. Commercial materials with the best accuracy in AM are not biocompatible, not biodegradable and lack bioactivity. Therefore, development of novel biomaterials for use in $\mathrm{AM}$ and improvement of $\mathrm{AM}$ techniques for currently available biomaterials are both required [170].

\section{Conclusions and Future Perspectives}

Polymer-based composites are highly promising materials to be used as biomedical implant materials for diverse biomedical applications. There are several advantages of using polymerbased composites, such as low cost of available natural and synthetic polymers and ease and tunability of manufacturing techniques. Several fillers with bioactive or non-bioactive properties hold opportunities in the engineering of suitable scaffolds or implants for tissue engineering and regenerative medicine applications.

The current techniques, applying polymer-based composites, need in general fine-tuning to optimize the structure required in the specific application. Polymer-based composite scaffolds already showed not only high cell adhesion, biocompatibility, and biodegradability but also outstanding bioactivities in terms of tissue formation, function, stimulation, survival and antibacterial properties during in vitro and in vivo experiments.

However, there are still challenges because of the limited choice of bioactive and functional fillers, control of the bioactive expression in the polymer matrix, and manufacturing techniques of suitable structures. Polymer-based composites should be adapted to the biological microenvironment, thereby accelerating tissue repair and regeneration. Studies on the relationship between the material properties of a specific polymer-based composite, its 
structure, physical and mechanical properties and biological response for specific biomedical applications remain necessary.

Two developing trends in the field of polymer-based composites for biomedical application are summarized below.

1) Combination of different functional fillers in a polymer matrix provides opportunities to enable multifunctional expression. Modugno et al. reported that the combined use of carbon nanomaterials, metal nanoparticles and polymers showed good results for diagnosis, imaging, therapy and theranostic applications, thanks to the extraordinary structural, optical, chemical and thermal properties of the resulting materials [171]. It is to be expected that more polymer/multifunctional filler composites will be developed as materials for the engineering or repair of tissues.

2) Polymers express tunable characteristics due to their wide range of physical and chemical properties. Some polymers show 'intelligent' characteristics such as stimuli-responsive behavior. This causes shape changes by stimulative factors such as temperature, humidity, solvent, $\mathrm{pH}$, light, and others. These controlled shape changes provide opportunities for the manufacturing of complex structures by so-called 4D printing, in which time is the fourth dimension. 4D printing uses AM technologies to fabricate stimuli-responsive 3D parts that can form novel structures when subjected to appropriate stimuli [172]. As this involves multimaterial printing, it is to be expected that polymer-based composites will be used in the 4D printing of complex and multifunctional structures for biomedical applications.

\section{References}

[1] L.L. Hench, Biomaterials: a forecast for the future, Biomaterials. 19 (1998) 1419-1423.

[2] Langer R, D.A. Tirrell, Designing materials for biology and medicine, Nature, 428(2004) 487-492.

[3] N.A. Peppas, R. Langer, New challenges in biomaterials, Science, 263 (1994) 1715-1720.

[4] S. Bose, D. Ke, H. Sahasrabudhe, A. Bandyopadhyay, Additive manufacturing of biomaterials, Prog. Mater. Sci., 93 (2018) 45-111.

[5] M.T. Lam, J.Wu, Biomaterial applications in cardiovascular tissue repair and regeneration, Expert Rev. Cardiovasc. Ther., 10 (2012) 1039-1049. 
[6] E. Caló, V.V. Khutoryanskiy, Biomedical applications of hydrogels: A review of patents and commercial products, Eur. Polym. J, 65 (2015) 252-267.

[7] N.S. Satarkar, D. Biswal, J.Z. Hilt, Hydrogel nanocomposites: a review of applications as remote controlled biomaterials, Soft Matter, 6 (2010) 2364-2371.

[8] J. Yang, G.L. Koons, G. Cheng, L. Zhao, A.G. Mikos, F. Cui, A review on the exploitation of biodegradable magnesium-based composites for medical applications, Biomed. Mater.,13 (2018) 022001.

[9] D.F. Williams, Challenges with the development of biomaterials for sustainable tissue engineering, Front BioEng. Biotech, 7 (2019) 127.

[10] F. Chen, X. Liu, Advancing biomaterials of human origin for tissue engineering, Prog. Polym. Sci., 53 (2016) 86-168.

[11] A.J. Teo, A. Mishra, I. Park, Y.J. Kim, W.T. Park, Y.J. Yoon, Polymeric biomaterials for medical implants and devices, ACS Biomatter. Sci. Eng., 2 (2016) 454-472.

[12] D.F. Stamatialis, B.J. Papenburg, M. Girones, S. Saiful, S.N. Bettahalli, S. Schmitmeier, M. Wessling, Medical applications of membranes: drug delivery, artificial organs and tissue engineering, J. Membr. Sci., 308 (2008) 1-34.

[13] J. Li, S. de Rosa, J. Wang, K. Zhang, Biomaterials Development, Modification, and Potential Application for Interventional Cardiology, BioMed. Res. Int., 2020 (2020) 4890483.

[14] M.H. Lee, J.A. Arcidiacono, A.M. Bilek, J.J. Wille, C.A. Hamill, K.M. Wonnacott, M. A. Wells, S. S. Oh, Considerations for tissue-engineered and regenerative medicine product development prior to clinical trials in the United States, Tissue Eng. Part B Rev., 16 (2009) 4154.

[15] A. Market, Global Industry Analysis, Size, Share, Growth, Trends, and Forecast 20172025, (2018),

[16] K.L. Christman, Biomaterials for tissue repair, Science, 363 (2019) 340-341.

[17] F. J.O'brien, Biomaterials \& scaffolds for tissue engineering, Mater. Today, 14 (2011) 8895.

[18] J. A. Hubbell, Biomaterials in tissue engineering, Bio/technology, 13 (1995) 565-576.

[19] R. Langer, J.P. Vacanti, Tissue engineering, Science, 260 (1993) 920-926. 
[20] S J Hollister, Porous scaffold design for tissue engineering, Nature Materials, 4 (2005) $518-524$.

[21] L.G. Griffith, G Naughton, Tissue engineering--current challenges and expanding opportunities, Science, 295 (2002) 1009-1014.

[22] F. Khan, M. Tanaka, Designing smart biomaterials for tissue engineering, Int. J. Mol. Sci., 19 (2018) 17.

[23] U. Jammalamadaka, K. Tappa, Recent advances in biomaterials for 3D printing and tissue engineering, J. Funct. Biomater., 9 (2018) 22.

[24] Z. Hao, Z. Song, J. Huang, K. Huang, A. Panetta, Z. Gu, J. Wu, The scaffold microenvironment for stem cell based bone tissue engineering, Biomater. Sci., 5 (2017): 13821392.

[25] M. Wang, Developing bioactive composite materials for tissue replacement, Mater. Sci. Eng., 85 (2018) 154-161.

[26] S. Pina, P R. Viviana, C F. Marques, F.R. Maia, T.H. Silva, R.L. Reis, J.M. Oliveira, Scaffolding strategies for tissue engineering and regenerative medicine applications, Materials, 12 (2019) 1824.

[27] Q. He, J. Zhang, Y. Liao, E.V. Alakpa, V. Bunpetch, H. Ouyang, Current advances in microsphere based cell culture and tissue engineering, Biotechnol. Adv., 39 (2019) 107459107459.

[28] S. Derakhshanfar, R. Mbeleck, K. Xu, X. Zhang, W. Zhong, M. Xing, 3D bioprinting for biomedical devices and tissue engineering: A review of recent trends and advances, Bioact. Mater., 3 (2018) 144-156.

[29] P. Zhao, H. Gu, H. Mi, C. Rao, J. Fu, L.S. Turng. Fabrication of scaffolds in tissue engineering: A review, Front. Mech. Eng., 13 (2018)107-119.

[30] A. Sionkowska, Current research on the blends of natural and synthetic polymers as new biomaterials. Prog. Polym. Sci., 36 (2011) 1254-1276.

[31] B. Dhandayuthapani, Y. Yoshida, T. Maekawa, D.S. Kumar, Polymeric scaffolds in tissue engineering application: a review, Int. J. Polym. Sci., 2011 (2011) 290602. 
[32] K. Prasad, O. Bazaka, M. Chua, M. Rochford, L. Fedrick, J. Spoor, R. Symes, M. Tieppo, C. Collins, A. Cao, D. Markwell, Metallic biomaterials: Current challenges and opportunities, Materials, 10 (2017) 884.

[33] Q. Chen, G.A Thouas, Metallic implant biomaterials, Mater. Sci. Eng. R Rep., 87 (2015) $1-57$.

[34] W. Wang, K.W. Yeung, Bone grafts and biomaterials substitutes for bone defect repair: A review, Bioact. Mater., 2 (2017) 224-247.

[35] C. Cha, S.R. Shin, N. Annabi, M.R. Dokmeci, A. Khademhosseini, Carbon-based nanomaterials: multifunctional materials for biomedical engineering, ACS Nano, 7 (2013) 2891-2897.

[36] R.A. Perez, J.E. Won, J.C. Knowles, H.W. Kim, Naturally and synthetic smart composite biomaterials for tissue regeneration, Adv. Drug Deliv. Rev., 65 (2013) 471-496.

[37] M.S. Shoichet, Polymer scaffolds for biomaterials applications, Macromolecules, 43 (2009) 581-591.

[38] J. Jagur-Grodzinski, Polymers for tissue engineering, medical devices, and regenerative medicine. Concise general review of recent studies, Polym. Adv. Technol., 17 (2006) 395-418. [39] E.S. Place, J.H. George, C.K. Williams, M.M. Stevens, Synthetic polymer scaffolds for tissue engineering. Chem. Soc. Rev., 38 (2009) 1139-1151.

[40] L. Lu, S.J. Peter, M.D. Lyman, H L. Lai, S.M. Leite, J.A. Tamada, S. Uyama, J.P. Vacanti, R. Langer, A.G. Mikos, In vitro and in vivo degradation of porous poly (DL-lactic-co-glycolic acid) foams, Biomaterials, 21 (2000) 1837-1845.

[41] H. Liu, E.B. Slamovich, T.J. Webster, Less harmful acidic degradation of poly (lactic-coglycolic acid) bone tissue engineering scaffolds through titania nanoparticle addition, Int. J. Nanomedicine, 1(2006) 541-545.

[42] K. Fukushima, Poly(trimethylene carbonate)-based polymers engineered for biodegradable functional biomaterials, Biomater. Sci., 4(2016) 9-24.

[43] Q. Hou, D W. Grijpma, J. Feijen, Creep-resistant elastomeric networks prepared by photocrosslinking fumaric acid monoethyl ester-functionalized poly(trimethylene carbonate) oligomers, Acta Biomater., 5(2009) 1543-1551. 
[44] S. Schüller-Ravoo, J. Feijen, D.W. Grijpma, Flexible, elastic and tear-resistant networks prepared by photo-crosslinking poly(trimethylene carbonate) macromers, Acta Biomater., 8 (2012) 3576-3585.

[45] S.B.G. Blanquer, S. Sharifi, D.W. Grijpma, Development of poly(trimethylene carbonate) network implants for annulus fibrosus tissue engineering, J. Appl. Biomater. Funct. Mater., 10 (2012) 177-184.

[46] A.C. Albertsson, M. Eklund, Influence of molecular structure on the degradation mechanism of degradable polymers: In vitro degradation of poly(trimethylene carbonate), poly(trimethylene carbonate-co-caprolactone), and poly(adipic anhydride). J. Appl. Polym. Sci., 57 (1995) 87-103.

[47] Z. Zhang, R. Kuijer, S.K. Bulstra, D.W. Grijpma, J. Feijen, The in vivo and in vitro degradation behavior of poly(trimethylene carbonate), Biomaterials, 27(2006)1741-1748.

[48] L. Yang, J. Li, W. Zhang, Y. Jin, J. Zhang, Y. Liu, D. Yi, M. Li, J. Guo, Z. Gu, The degradation of poly (trimethylene carbonate) implants: The role of molecular weight and enzymes, Polym. Degrad. Stab., 122(2015) 77-87.

[49] Z. Zhang, S. Zou, G.J. Vancso, D.W. Grijpma, J, Feijen, Enzymatic surface erosion of poly (trimethylene carbonate) films studied by atomic force microscopy, Biomacromolecules, 6 (2005) 3404-3409.

[50] J.J. Rongen, B. van Bochove, G. Hannink, D.W. Grijpma, P. Buma, Degradation behavior of, and tissue response to photo-crosslinked poly(trimethylene carbonate) networks, J. Biomed. Mater. Res. A, 104 (2016) 2823-2832.

[51] B. van Bochove, D.W. Grijpma, Photo-crosslinked synthetic biodegradable polymer networks for biomedical applications, J. Biomater. Sci. Polym. Ed., 30 (2019) 77-106.

[52] M. Huber, G. Reinisch, G. Trettenhahn, K. Zweymüller, F. Lintner, Presence of corrosion products and hypersensitivity-associated reactions in periprosthetic tissue after aseptic loosening of total hip replacements with metal bearing surfaces, Acta Biomater., 5 (2009) 172180.

[53] M.A. Hussein, A.S. Mohammed, N. Al-Aqeeli, Wear characteristics of metallic biomaterials: a review, Materials, 8 (2015) 2749-2768. 
[54] P. Thoniyot, M.J. Tan, A.A. Karim, D.J. Young, X.J. Loh, Nanoparticle-hydrogel composites: Concept, design, and applications of these promising, multi-functional materials, Adv. Sci., 2 (2015) 1400010.

[55] Q.Z. Chen, I.D. Thompson, A.R. Boccaccini, 45S5 Bioglass ${ }^{\circledR}-$ derived glass-ceramic scaffolds for bone tissue engineering, Biomaterials, 27 (2006) 2414-2425.

[56] S. Samavedi, A.R. Whittington, A.S. Goldstein, Calcium phosphate ceramics in bone tissue engineering: a review of properties and their influence on cell behavior, Acta Biomater., 9 (2013) 8037-8045.

[57] H. Yuan, H. Fernandes, P. Habibovic, J. De Boer, AM. Barradas, A. De Ruiter, WR. Walsh, CA. Van Blitterswijk, JD. De Bruijn, Osteoinductive ceramics as a synthetic alternative to autologous bone grafting. Proc. Natl. Acad. Sci. U.S.A, 107(2010) 13614-13619.

[58] R. Eivazzadeh-Keihan, A. Maleki, M. De La Guardia, M.S. Bani, K.K. Chenab, P. Pashazadeh-Panahi, Baradaran B, A. Mokhtarzadeh, MR. Hamblin, Carbon based nanomaterials for tissue engineering of bone: Building new bone on small black scaffolds: A review, J. Adv. Res., 18, (2019) 185-201.

[59] Y. Yang, A.M. Asiri, Z. Tang, D. Du, Y. Lin, Graphene based materials for biomedical applications, Mater. Today, 16, (2013) 365-373.

[60] C. Cha, S.R. Shin, N. Annabi, M.R. Dokmeci, A. Khademhosseini, Carbon-based nanomaterials: multifunctional materials for biomedical engineering, ACS nano, 7 (2013) 2891-2897.

[61] S. Ramakrishna, J. Mayer, E. Wintermantel, K.W. Leong, Biomedical applications of polymer-composite materials: a review, Compos. Sci. Technol., 61 (2001) 1189-1224.

[62] E. Salernitano, C. Migliaresi, Composite materials for biomedical applications: a review, J. Appl. Biomech., 1 (2003) 3-18.

[63] N. Zeng, A.C. van Leeuwen, D.W. Grijpma, R.R. Bos, R. Kuijer, Poly(trimethylene carbonate)-based composite materials for reconstruction of critical-sized cranial bone defects in sheep, J. Craniomaxillofac. Surg., 45 (2017) 338-346.

[64] X. Liang, Y. Qi, Z. Pan, Y. He, X. Liu, S. Cui, J. Ding, Design and preparation of quasispherical salt particles as water-soluble porogens to fabricate hydrophobic porous scaffolds for tissue engineering and tissue regeneration, Mater. Chem. Front., 2 (2018) 1539-1553. 
[65] M.M. Stevens, Biomaterials for bone tissue engineering, Mater. Today, 11 (2008) 18-25.

[66] L.L. Hench, The story of Bioglass ${ }^{\circledR}$, J. Mater. Sci. Mater. Med., 17 (2006) 967-978.

[67] A.M. Ferreira, P. Gentile, V. Chiono, G. Ciardelli, Collagen for bone tissue regeneration, Acta Biomater., 8 (2012) 3191-3200.

[68] K. Rez wan, Q.Z. Chen, J.J. Blaker, A.R. Boccaccini, Biodegradable and bioactive porous polymer/inorganic composite scaffolds for bone tissue engineering, Biomaterials, 2006 (18) 3413-3431.

[69] S. Samavedi, A.R. Whittington, A.S. Goldstein, Calcium phosphate ceramics in bone tissue engineering: a review of properties and their influence on cell behavior, Acta Biomater, 9 (2013) 8037-8045.

[70] A.J.W. Johnson, B.A. Herschler, A review of the mechanical behavior of $\mathrm{CaP}$ and $\mathrm{CaP} /$ polymer composites for applications in bone replacement and repair. Acta Biomaterialia, 7(2011) 16-30.

[71] A.R. Boccaccini, M. Erol, W.J. Stark, D. Mohn, Z. Hong, J.F. Mano, Polymer/bioactive glass nanocomposites for biomedical applications: a review, Compos. Sci. Technol., 70(2010) 1764-1776.

[72] Z. Hong, R.L. Reis, J.F. Mano, Preparation and in vitro characterization of novel bioactive glass ceramic nanoparticles, J. Biomed. Mater. Res. A, 88 (2009) 304-313.

[73] Z. Hong, R.L. Reis, J.F. Mano, Preparation and in vitro characterization of scaffolds of poly(L-lactic acid) containing bioactive glass ceramic nanoparticles, Acta Biomater., 4 (2008) 1297-1306.

[74] N. Ramesh, S.C Moratti, G.J. Dias, Hydroxyapatite-polymer biocomposites for bone regeneration: A review of current trends, J. Biomed. Mater. Res. Part B Appl. Biomater, 106 (2018) 2046-2057.

[75] S. Hassanajili, A. Karami-Pour, A. Oryan, T. Talaei-Khozani, Preparation and characterization of PLA/PCL/HA composite scaffolds using indirect 3D printing for bone tissue engineering, Mater. Sci. Eng. C, 104 (2019) 109960.

[76] O. Guillaume, M.A. Geven, C.M. Sprecher, V.A. Stadelmann, D.W. Grijpma, T. Tang, L. Qin, Y. Lai, M. Alini, J.D. de Bruijn, H. Yuan, Surface-enrichment with hydroxyapatite 
nanoparticles in stereolithography-fabricated composite polymer scaffolds promotes bone repair, Acta Biomater., 54 (2017) 386-398.

[77] J. Park, S.J. Lee, H.H. Jo, J.H. Lee, W.D. Kim, J.Y. Lee, A. Su , Fabrication and characterization of 3D-printed bone-like $\beta$-tricalcium phosphate/polycaprolactone scaffolds for dental tissue engineering, J. Ind. Eng. Chem., 46 (2017) 175-181.

[78] S. Konopnicki, B. Sharaf, C. Resnick, A, Patenaude, T, Pogal-Sussman, K.G, Hwang, H, Abukawa, M.J. Troulis, Tissue-engineered bone with 3-dimensionally printed $\beta$-tricalcium phosphate and polycaprolactone scaffolds and early implantation: An in vivo pilot study in a porcine mandible model, J. Oral Maxillofac. Surg., 73 (2015) 1016.e1-1016.e11.

[79] Y.M. Shin, J.S. Park, S.I. Jeong, S.J. An, H.J. Gwon, Y.M. Lim, Y.C. Nho, C.Y. Kim, Promotion of human mesenchymal stem cell differentiation on bioresorbable polycaprolactone/biphasic calcium phosphate composite scaffolds for bone tissue engineering, Biotechnol. Bioprocess Eng., 19 (2014) 341-349.

[80] M. Peroglio, L. Gremillard, C. Gauthier, L. Chazeau, S. Verrier, M. Alini, J. Chevalier, Mechanical properties and cytocompatibility of poly ( $\varepsilon$-caprolactone)-infiltrated biphasic calcium phosphate scaffolds with bimodal pore distribution, Acta Biomater., 11 (2010) 43694379.

[81] Z. Ma, F. Chen, Y. Zhu, T. Cui, X. Liu, Amorphous calcium phosphate/poly(D, L-lactic acid) composite nanofibers: electrospinning preparation and biomineralization, J. Colloid Interface Sci., 359 (2011) 371-379.

[82] X. Ye, L. Li, Z. Lin, W. Yang, M. Duan, L. Chen, Y. Lu, Y. Zhang, Integrating 3D-printed PHBV/Calcium sulfate hemihydrate scaffold and chitosan hydrogel for enhanced osteogenic property, Carbohydr. Polym, 202 (2018)106-114.

[83] Z. Cao, D. Wang, L. Lyu, Y. Gong, Y. Li, Fabrication and characterization of PCL/CaCO 3 electrospun composite membrane for bone repair, RSC Adv, 6(2016) 10641-10649.

[84] AG. Castro, M. Diba, M. Kersten, JA. Jansen, JJ. van den Beucken, F. Yang, Development of a PCL-silica nanoparticles composite membrane for guided bone regeneration, Mater. Sci. Eng. C, 85(2018) 154-161.

[85] S. Tamburaci, F. Tihminlioglu, Biosilica incorporated 3D porous scaffolds for bone tissue engineering applications, Mater. Sci. Eng. C, 91(2018) 274-291. 
[86] B. Guo, PX. Ma, Conducting polymers for tissue engineering, Biomacromolecules, 19 (2018)1764-82.

[87] A. Saberi, F. Jabbari, P. Zarrintaj, MR. Saeb, M. Mozafari, Electrically Conductive Materials: Opportunities and Challenges in Tissue Engineering, Biomolecules, 9(2019) 448.

[88] H. Cui, J. Shao, Y. Wang, P. Zhang, X. Chen, Y. Wei, PLA-PEG-PLA and its electroactive tetraaniline copolymer as multi-interactive injectable hydrogels for tissue engineering, Biomacromolecules, 14(2013)1904-1912.

[89] R. Balint, NJ. Cassidy, SH. Cartmell, Conductive polymers: Towards a smart biomaterial for tissue engineering, Acta Biomater, 10(2014) 2341-2353.

[90] JO. You, M. Rafat, GJ. Ye, DT. Auguste, Nanoengineering the heart: conductive scaffolds enhance connexin 43 expression, Nano Lett, 11(2011)3643-3648.

[91] P. Zarrintaj, S. Manouchehri, Z. Ahmadi, MR. Saeb, AM. Urbanska, DL. Kaplan, M. Mozafari, Agarose-based biomaterials for tissue engineering, Carbohydr. Polym, 187 (2018) 66-84.

[92] G. Jin, K. Li, The electrically conductive scaffold as the skeleton of stem cell niche in regenerative medicine, Mater. Sci. Eng. C, 45 (2014)671-681.

[93] M. Gajendiran, J. Choi, SJ. Kim, K. Kim, H. Shin, HJ. Koo, K. Kim, Conductive biomaterials for tissue engineering applications, J. Ind. Eng. Chem, 51 (2017) 12-26.

[94] L. Ghasemi-Mobarakeh, M.P. Prabhakaran, M. Morshed, M.H. Nasr-Esfahani, H. Baharvand, S. Kiani, SS. Al-Deyab, S. Ramakrishna, Application of conductive polymers, scaffolds and electrical stimulation for nerve tissue engineering, J. Tissue Eng. Regen. Med., 5 (2011) e17-e35.

[95] R. Balint, N.J. Cassidy, S.H Cartmell, Conductive polymers: Towards a smart biomaterial for tissue engineering, Acta Biomater., 10 (2014) 2341-2353.

[96] G. Kaur, R. Adhikari, P. Cass, M. Bown, P. Gunatillake, Electrically conductive polymers and composites for biomedical applications, RSC Adv., 5 (2015) 37553-37567.

[97] P.J. Brigandi, J.M. Cogen, R.A. Pearson, Electrically conductive multiphase polymer blend carbon - based composites, Polym. Eng. Sci., 54 (2014) 1-16. 
[98] T. Gurunathan, C.R. Rao, R. Narayan, K.V. Raju, Polyurethane conductive blends and composites: synthesis and applications perspective, J. Mater. Sci, 48 (2013) 67-80.

[99] S.W. Crowder, Y. Liang, R. Rath, AM. Park, S. Maltais, P.N. Pintauro, W. Hofmeister, C.C. Lim, X. Wang, H.J. Sung, Poly( $\varepsilon$-caprolactone)-carbon nanotube composite scaffolds for enhanced cardiac differentiation of human mesenchymal stem cells, Nanomedicine, 8 (2013) 1763-1776.

[100] Z. Zhou, X. Liu, W. Wu, S. Park, A.L. Miller Ii, A. Terzic, L. Lu, Effective nerve cell modulation by electrical stimulation of carbon nanotube embedded conductive polymeric scaffolds, Biomater Sci., 6 (2018) 2375-2385.

[101] S. Sayyar, E. Murray, B.C. Thompson, S. Gambhir, D.L. Officer, G.G. Wallace, Covalently linked biocompatible graphene/polycaprolactone composites for tissue engineering, Carbon 52 (2013) 296-304.

[102] S.R. Shin, C. Zihlmann, M. Akbari, P. Assawes, L. Cheung, K. Zhang, V. Manoharan, Y.S. Zhang, M. Yüksekkaya, K.T. Wan, M. Nikkhah, Reduced graphene oxide-gelMA hybrid hydrogels as scaffolds for cardiac tissue engineering, Small, 12 (2016) 3677-3689.

[103] W. Chen, S. Zhang, Y. Yu, H. Zhang, Q. He, Structural-engineering rationales of gold nanoparticles for cancer theranostics, Adv. Mater., 28 (2016) 8567-8585.

[104] S. Vial, R.L. Reis, J.M. Oliveira, Recent advances using gold nanoparticles as a promising multimodal tool for tissue engineering and regenerative medicine, Curr. Opin. Solid State Mater. Sci., 21(2017)92-112.

[105] A. Navaei, H. Saini, W. Christenson, R.T. Sullivan, R. Ros, M. Nikkhah, Gold nanorodincorporated gelatin-based conductive hydrogels for engineering cardiac tissue constructs, Acta Biomater., 41 (2016) 133-146.

[106] M. Shevach, B.M. Maoz, R. Feiner, A. Shapira, T. Dvir, Nanoengineering gold particle composite fibers for cardiac tissue engineering, J. Mater. Chem. B, 1 (2013) 5210-5217.

[107] V.F. Cardoso, A. Francesko, C. Ribeiro, M. Bañobre-López, P. Martins, S. LancerosMendez, Advances in magnetic nanoparticles for biomedical applications, Adv. Healthc. Mater., 7 (2018)1700845. 
[108] G. Lin, D. Makarov, O.G. Schmidt, Magnetic sensing platform technologies for biomedical applications, Lab on a Chip, 17 (2017) 1884-1912.

[109] N. Tran, T.J. Webster, Magnetic nanoparticles: biomedical applications and challenges, J. Mater. Chem., 20 (2010) 8760-8767.

[110] X. Li, J. Wei, KE. Aifantis, Y. Fan, Q. Feng, F.Z. Cui, F. Watari, Current investigations into magnetic nanoparticles for biomedical applications, J. Biomed. Mater. Res. A, 104 (2016) 1285-1296.

[111] N. Bock, A. Riminucci, C. Dionigi, A. Russo, A. Tampieri, E. Landi, V.A. Goranov, M. Marcacci, V. Dediu, A novel route in bone tissue engineering: magnetic biomimetic scaffolds, Acta Biomater., 6 (2010) 786-796.

[112] M. Bañobre-López, Y. Piñeiro-Redondo, R. De Santis, A. Gloria, L. Ambrosio, Anna Tampieri, V. Dediu, J. Rivas, Poly(caprolactone) based magnetic scaffolds for bone tissue engineering, J. Appl. Phys., 109 (2011) 07B313.

[113] H. Zhang, J. Xia, Pang X, M. Zhao, B. Wang, L. Yang, H. Wan, J. Wu, S. Fu, Magnetic nanoparticle-loaded electrospun polymeric nanofibers for tissue engineering, Mater. Sci. Eng. C,73 (2017)537-543.

[114] Q. Cai, Y. Shi, D. Shan, W. Jia, S. Duan, X. Deng, X. Yang, Osteogenic differentiation of MC3T3-E1 cells on poly (1-lactide)/Fe3O4 nanofibers with static magnetic field exposure. Mater. Sci. Eng. C, 55 (2015)166-173.

[115] S. Panseri, A. Russo, M. Sartori, G. Giavaresi, M. Sandri, M. Fini, M.C. Maltarello, T. Shelyakova, A. Ortolani, A. Visani, V. Dediu, Modifying bone scaffold architecture in vivo with permanent magnets to facilitate fixation of magnetic scaffolds, Bone, 56 (2013)432-439.

[116] S. Gil, J.F. Mano, Magnetic composite biomaterials for tissue engineering, Biomater. Sci, 2 (2014) 812-818. https://doi.org/10.1039/C4BM00041B

[117] M. Gholipourmalekabadi, S. Zhao, B.S. Harrison, M. Mozafari, A.M. Seifalian, Oxygengenerating biomaterials: a new, viable paradigm for tissue engineering, Trends Biotechnol., 34 (2016) 1010-1021.

[118] G. Camci-Unal, N. Alemdar, N. Annabi, A. Khademhosseini, Oxygen-releasing biomaterials for tissue engineering, Polym. Int., 62 (2013) 843-848. https://doi.org/10.1002/pi.4502 
[119] S. Suvarnapathaki, X. Wu, D. Lantigua, M.A. Nguyen, G. Camci-Unal, Breathing life into engineered tissues using oxygen-releasing biomaterials, NPG Asia Mater., 11 (2019) 1-18.

[120] E. Pedraza, M.M. Coronel, C.A. Fraker, C. Ricordi, C.L. Stabler, Preventing hypoxiainduced cell death in beta cells and islets via hydrolytically activated, oxygen-generating biomaterials, Proc. Natl. Acad. Sci. U.S.A, 109 (2012) 4245-4250.

[121] H. Steg, A.T. Buizer, W. Woudstra, A.G. Veldhuizen, S.K. Bulstra, D.W. Grijpma, R. Kuijer, Control of oxygen release from peroxides using polymers, J. Mater. Sci. Mater. Med., 26 (2015) 207.

[122] H. Steg, A.T. Buizer, W. Woudstra, A.G. Veldhuizen, S.K. Bulstra, D.W. Grijpma, R. Kuijer, Oxygen-releasing poly (trimethylene carbonate) microspheres for tissue engineering applications, Polym. Adv. Technol., 28(2017) 1252-1257.

[123] B.S. Harrison, D. Eberli, S.J. Lee, A. Atala, J.J. Yoo, Oxygen producing biomaterials for tissue regeneration, Biomaterials, 28 (2007) 4628-4634.

[124] P.A. Shiekh, A. Singh, A. Kumar, Oxygen-releasing antioxidant cryogel scaffolds with sustained oxygen delivery for tissue engineering applications, ACS Appl. Mater. Interfaces, 10 (2018) 18458-18469.

[125] Z. Fan, Z. Xu, H. Niu, N. Gao, Y. Guan, C. Li, Y. Dang, X. Cui, XL. Liu, Y. Duan, H. $\mathrm{Li}$, An injectable oxygen release system to augment cell survival and promote cardiac repair following myocardial infarction, Sci. Rep., 8 (2018) 1371.

[126] J.A. Lichter, K.J. van Vliet, M.F. Rubner, Design of antibacterial surfaces and interfaces: polyelectrolyte multilayers as a multifunctional platform, Macromolecules, 42 (2009) 8573 8586.

[127] D. Sun, M. Babar Shahzad, M. Li, G. Wang, D. Xu, Antibacterial materials with medical applications, Mater. Technol., 30 (2015) B90-B95.

[128] K. Vasilev, J. Cook, H.J. Griesser, Antibacterial surfaces for biomedical devices, Expert Rev. Med. Devices, 6 (2009) 553-567.

[129] M. Katsikogianni, Y.F. Missirlis, Concise review of mechanisms of bacterial adhesion to biomaterials and of techniques used in estimating bacteria-material interactions, Eur. Cell. Mater., 8 (2004) 37-57. 
[130] C.T. Johnson, A.J. García, Scaffold-based anti-infection strategies in bone repair, Ann. Biomed. Eng., 43 (2015) 515-528.

[131] Q. Yun'an, L. Cheng, R. Li, G. Liu, Y. Zhang, X. Tang, J. Wang, H. Liu, Y. Qin, Potential antibacterial mechanism of silver nanoparticles and the optimization of orthopedic implants by advanced modification technologies, Int. J. Nanomedicine, 13 (2018) 3311.

[132] Y. Zare, I. Shabani, Polymer/metal nanocomposites for biomedical applications, Mater. Sci. Eng. C, 60 (2016) 195-203.

[133] K. Vimala, KS. Sivudu, YM. Mohan, B. Sreedhar, KM. Raju, Controlled silver nanoparticles synthesis in semi-hydrogel networks of poly(acrylamide) and carbohydrates: a rational methodology for antibacterial application, Carbohydr. Polym., 75 (2009) 463-471.

[134] E. Fortunati, L. Latterini, S. Rinaldi, J.M. Kenny, I.P. Armentano, PLGA/Ag nanocomposites: in vitro degradation study and silver ion release, J. Mater. Sci. Mater. Med., 22 (2011) 2735-2744.

[135] M.E. Samberg, P. Mente, T. He, M.W. King, N.A. Monteiro-Riviere, In vitro biocompatibility and antibacterial efficacy of a degradable poly(L-lactide-co-epsiloncaprolactone) copolymer incorporated with silver nanoparticles, Ann. Biomed. Eng., 42 (2014) 1482-1493.

[136] R.V. Madhavan, M.J. Rosemary, M.A. Nandkumar, K.V. Krishnan, L.K. Krishnan, Silver nanoparticle impregnated poly(e-caprolactone) scaffolds: optimization of antibacterial and noncytotoxic concentrations, Tissue Eng. A, 17 (2010) 439-449.

[137] H.R. Bakhsheshi-Rad, A.F. Ismail, M. Aziz, Z. Hadisi, M. Omidi, X. Chen, Antibacterial activity and corrosion resistance of $\mathrm{Ta}_{2} \mathrm{O}_{5}$ thin film and electrospun PCL/MgO-Ag nanofiber coatings on biodegradable Mg alloy implants, Ceram. Int., 45 (2019) 11883-11892.

[138] H. Rodrígues-Tobías, G. Morales, A. Ledezma, J. Romero, D. Grande, Novel antibacterial electrospun mats based on poly(D,L-lactide) nanofibers and zinc oxide particles, J. Mater. Sci., 49 (2014) 8373-8385.

[139] K. Krishnamoorty, G. Manivannan, S.J. Kim, K. Jeyasubramanian, M. Premanathan, Antibacterial activity of $\mathrm{MgO}$ nanoparticles based on lipid peroxidation by oxygen vacancy, $\mathrm{J}$. Nanopart. Res., 14 (2012) 1063-1066. 
[140] V. Tiwari, N. Mishra, K. Gadani, P.S. Solanki, N.A. Shah, M. Tiwari, Mechanism of anti-bacterial activity of zinc oxide nanoparticle against carbapenem-resistant acinetobacter baumannii, Front. Microbiol., 9 (2018) 1218.

[141] X. Zhang, M.A. Geven, D.W. Grijpma, J.E. Gautrot, T. Peijs, Polymer-polymer composites for the design of strong and tough degradable biomaterials, Mater. Today Commun, 8 (2016) 53-63.

[142] S.J. Hollister, Porous scaffold design for tissue engineering, Nature Mater., 4 (2005) 518524.

[143] H. Janik, M. Marzec, A review: Fabrication of porous polyurethane scaffolds, Mater. Sci. Eng. C, 48 (2015) 586-591.

[144] Q. Hou, D.W. Grijpma, J. Feijen, Porous polymeric structures for tissue engineering prepared by a coagulation, compression moulding and salt leaching technique, Biomaterials, 24 (2003) 1937-1947.

[145] Y. Song, JW. Wennink, AA. Poot, I. Vermes, J. Feijen, DW. Grijpma, Evaluation of tubular poly (trimethylene carbonate) tissue engineering scaffolds in a circulating pulsatile flow system, Int J Artif Organs, 34 (2011) 161-171.

[146] WL. Murphy, RG. Dennis, JL. Kileny, DJ. Mooney, Salt fusion: an approach to improve pore interconnectivity within tissue engineering scaffolds, Tissue Eng Part A, 8 (2002) 43-52.

[147] X. Liang, Y. Qi, Z. Pan, Y. He, X. Liu, S. Cui, J. Ding, Design and preparation of quasispherical salt particles as water-soluble porogens to fabricate hydrophobic porous scaffolds for tissue engineering and tissue regeneration, Mater. Chem. Front, 2 (2018) 1539-1553.

[148] J. Stampfl, S. Baudis, C. Heller, R. Liska, A. Neumeister, R. Kling, A. Ostendorf, M. Spitzbart, Photopolymerization with tunable mechanical properties processed by laser-based high-resolution stereolithography, J. Micromech. Microeng, 18(2008) 125014.

[149] SC. Ligon, R. Liska, J. Stampfl, M. Gurr, R. Mülhaupt, Polymers for 3D printing and customized additive manufacturing, Chem. Rev, 117(2017) 10212-10290.

[150] X. Mu, T. Bertron, C. Dunn, H. Qiao, J. Wu, Z. Zhao, C. Saldana, H. Qi, Porous polymeric materials by 3D printing of photocurable resin, Mater. Horiz, 4 (2017) 442-449. 
[151] V. Raeisdasteh Hokmabad, S. Davaran, A. Ramazani, R. Salehi, Design and fabrication of porous biodegradable scaffolds: a strategy for tissue engineering, J. Biomater. Sci. Polym. Ed, 28 (2017) 1797-1825.

[152] A. Eltom, G. Zhong, A. Muhammad, Scaffold Techniques and Designs in Tissue Engineering Functions and Purposes: A Review, Adv. Mater. Sci. Eng, 2019 (2019) 3429527.

[153] Y. Song, MM. Kamphuis, Z. Zhang, LT. Sterk, I. Vermes, AA. Poot, J. Feijen, DW. Grijpma, Flexible and elastic porous poly (trimethylene carbonate) structures for use in vascular tissue engineering, Acta Biomater, 6 (2010) 1269-1277.

[154] NH. Ngadiman, MY. Noordin, A. Idris, D. Kurniawan, A review of evolution of electrospun tissue engineering scaffold: From two dimensions to three dimensions, Proc. Inst. Mech. Eng. H, 231 (2017)

[155] A.P. Kishan, E.M. Cosgriff-Hernandez, Recent advancements in electrospinning design for tissue engineering applications: a review, J. Biomed. Mater. Res, 105 (2017) 2892-2905.

[156] I. Armentano, M. Dottori, E. Fortunati, S. Mattioli, J.M. Kenny, Biodegradable polymer matrix nanocomposites for tissue engineering: a review, Polym. Degrad. Stab, 95 (2010) 21262146.

[157] W. Yu, X. Jiang, M. Cai, W. Zhao, D. Ye, Y. Zhou, C. Zhu, X. Zhang, X. Lu, Z. Zhang, A novel electrospun nerve conduit enhanced by carbon nanotubes for peripheral nerve regeneration, Nanotechnology, 25 (2014) 165102.

[158] B. Chaudhuri, B. Mondal, S. Kumar, S.C. Sarkar, Myoblast differentiation and protein expression in electrospun graphene oxide (GO)-poly( $\varepsilon$-caprolactone, PCL) composite meshes, Mater. Lett., 182 (2016) 194-197.

[159] S. Khorshidi, A. Solouk, H. Mirzadeh, S. Mazinani, J.M. Lagaron, S. Sharifi, S. Ramakrishna, A review of key challenges of electrospun scaffolds for tissue engineering applications, J. Tissue Eng. Regen. Med., 10 (2016) 715-738.

[160] U. Jammalamadaka, K. Tappa, Recent advances in biomaterials for 3D printing and tissue engineering, J. Funct. Biomater., 9 (2018) 22.

[161] A.E. Jakus, A.L. Rutz, R.N. Shah, Advancing the field of 3D biomaterial printing, Biomed. Mater., 11 (2016) 014102. 
[162] J. Li, L. Zhang, S. Lv, S. Li, N. Wang, Z. Zhang, Fabrication of individual scaffolds based on a patient-specific alveolar bone defect model, J. Biotechnol, 151 (2011) 87-93.

[163] A. Youssef, S.J. Hollister, P.D. Dalton, Additive manufacturing of polymer melts for implantable medical devices and scaffolds, Biofabrication, 9 (2017) 012002.

[164] M.M. Zagho, E.A. Hussein, A.A. Elzatahry, Recent overviews in functional polymer composites for biomedical applications, Polymers, 10 (2018) 739.

[165] U. Kalsoom, P.N. Nesterenko, B, Paull, Recent developments in 3D printable composite materials, RSC. Adv., 6 (2016) 60355-60371.

[166] S.M. Peltola, F.P. Melchels, D.W. Grijpma, M. Kellomäki, A review of rapid prototyping techniques for tissue engineering purposes, Ann. Med, 40 (2008) 268-280.

[167] F.P. Melchels, J. Feijen, D.W. Grijpma, A review on stereolithography and its applications in biomedical engineering, Biomaterials, 31 (2010) 6121-6130.

[168] K.E.G. Dienel, B. van Bochove, J.V. Seppälä, Additive manufacturing of bioactive poly(trimethylene carbonate)/ $\beta$-tricalcium phosphate composites for bone regeneration, Biomacromolecules, 21 (2019) 366-375.

[169] O. Guillaume, M.A. Geven, D.W. Grijpma, T. Tang, L. Qin, Y. Lai, H. Yuan, R.G. Richards, D. Eglin, Poly(trimethylene carbonate) and nano-hydroxyapatite porous scaffolds manufactured by stereolithography, Polym. Adv. Technol., 28 (2017) 1219-1225.

[170] A.A. Zadpoor, J. Malda, Additive Manufacturing of Biomaterials, Tissues, and Organs, Ann Biomed Eng., 45 (2017)1-11.

[171] G. Modugno, C. Ménard-Moyon, M. Prato, A. Bianco, Carbon nanomaterials combined with metal nanoparticles for theranostic applications, Br. J. Pharmacol., 172 (2015) 975-991.

[172] M. Rafiee, R.D. Farahani, D. Therriault, Multi-Material 3D and 4D Printing: A Survey, Adv. Sci., 7 (2020) 1902307. 


\section{Chapter 3 - Preparation and characterization of poly(trimethylene carbonate) and reduced graphene oxide composites for nerve regeneration}

Stijn G. Rotman, Zhengchao Guo, Dirk W. Grijpma, André A. Poot

Department of Biomaterials Science and Technology, University of Twente, Enschede, The Netherlands.

Published

S.G. Rotman, Z. Guo, D.W. Grijpma, A.A. Poot, Preparation and characterization of poly(trimethylene carbonate) and reduced graphene oxide composites for nerve regeneration, Polym. Adv. Technol., 28 (2017) 1233-1238. 


\begin{abstract}
Although peripheral nerves are known to have some regenerative abilities, completely separated nerve ends are rarely known to restore function without medical intervention. The main scientific focus for novel treatments of peripheral nerve injury concentrates on a biodegradable nerve guide channel (NGC) that promotes and guides axonal regrowth. Because electrically conductive NGCs promote axon regeneration, we propose a composite material consisting of the polymer poly(trimethylene carbonate) (PTMC) and the conductive filler reduced graphene oxide (rGO). Graphite was oxidized following Hummers' method, exfoliated using sonication and subsequently reduced with hydrazine in order to form thin sheets of rGO. Three-armed PTMC functionalized with methacrylic anhydride (MA) was dissolved in dispersions of rGO in dimethylformamide with desired rGO contents. After precipitation and solvent casting in chloroform, the films were crosslinked under UV light. The PTMC-MA/rGO composite films with $0,0.5,1,2$ and $4 \mathrm{wt} \%$ showed increasing electroconductivity with values up to $6.88 * 10^{-2} \mathrm{~S} / \mathrm{cm}$. Culturing of PC-12 cells showed that after initial cell adhesion, the cells proliferated on the surface of the PTMC-MA/rGO composite films, regardless of the amount of rGO loading. The results imply that these PTMC-MA/rGO composites show promise toward their use in the fabrication of NGCs. The electrically conductive materials are expected to increase the rate of axonal regeneration, resulting in improved functional recovery of transected peripheral nerves.
\end{abstract}

\title{
1 Introduction
}

In comparison with the central nervous system, the peripheral nervous system is relatively permissive to axonal regrowth[1]. However, many factors like scar formation and unguided axonal regrowth contribute to incomplete regeneration and thus a failed restoration of function to the nerve. Current surgical interventions include end-to-end suturing for small nerve defects and a nerve autograft for nerve defects larger than $3 \mathrm{~cm} \mathrm{[2].} \mathrm{The} \mathrm{use} \mathrm{of} \mathrm{both} \mathrm{methods} \mathrm{is} \mathrm{limited}$ because of strain applied on sutured nerves and limited amount of nerve tissue that can be harvested for a nerve autograft. To prevent scar tissue ingrowth and to guide axonal regrowth, an implantable nerve guide channel (NGC) has been proposed. These NGCs should possess biocompatible and biodegradable properties to prevent host responses and a second invasive surgery to remove the NGC. The swelling properties of the NGC have to be minimal in order to prevent compression of the newly regenerated nerve. A major aspect that has proven to 
contribute to accelerated axonal growth is electrostimulation (ES) of the restoring nerve [3]. ES has been applied for tissue engineering purposes to trigger or promote certain cell behavior [4]. The availability of an NGC that would be able to conduct the bioelectricity that is present in the body could have substantial influence on the time necessary to restore function to the damaged peripheral nerve. Composites consisting of a polymer that exhibits optimal degradation and physical properties and a conductive filler that would allow the composite to conduct (bio)electricity are promising materials for the preparation of electrically conductive NGCs.

PTMC is a biocompatible, biodegradable and amorphous polymer with high flexibility [5]. Biomedical applications, either applied or still under development, include implants to support bone, cartilage or blood vessel regeneration [6]. The mechanical properties and degradation kinetics of PTMC are highly tunable by adjusting the molecular weight of the polymer chain or by synthesizing co-polymers with other monomer units like $\varepsilon$-caprolactone [7]. PTMC, as a hydrophobic polymer, has low water uptake and thus shows a low degree of swelling in water $[8]$.

Graphene is a single carbon layer orientated in a honeycomb lattice structure with $\mathrm{sp}^{2}$ hybridized carbon atoms. Pristine graphene is known to have excellent conductive properties due to the non-localized $\pi$-electrons of the $\mathrm{sp}^{2}$-hybridized carbon atoms that can move relatively freely over the graphene layer. The aspect ratio of graphene can rise to great values, dependent on the method of preparation. These properties give graphene a very low electrical percolation threshold when graphene is evenly dispersed in a composite system [9].

This study focused on the preparation of composites of methacrylic anhydride (MA) functionalized PTMC macromers and reduced graphene oxide (rGO) (PTMC-MA/rGO), containing varying amounts of rGO. The conductive properties of the composites and the interaction with water were evaluated. PC-12 cells, originating from rat pheochromocytoma, were cultured on PTMC-MA/rGO films to validate proper cell adhesion and proliferation on the composites. The results will give insights in the applicability of PTMC-MA/rGO composites as a material for NGC fabrication. 


\section{Materials and Methods}

\subsection{Materials}

Trimethylolpropane, ammonium persulfate, phorphorus pentoxide, potassium permanganate, sulphuric acid (>98\%), hydrogen peroxide solution (30\% in water), hydrogen chloride solution (37\%), stannous octoate, hydroquinone, methacrylic anhydride, Irgacure 2959 and triethylamine were purchased from Sigma Aldrich, Netherlands and were used as delivered. Fine graphite flakes were acquired from Alfa Aesar, Germany. Dichloromethane (>99.5\%), chloroform (>99\%) and ethanol (98\%) were acquired from VWR chemicals, Belgium. Dimethylformamide $(>99.5 \%)$ was purchased from Biosolve, Netherlands. TMC monomer was kindly provided by Huizhou Foryou Medical Devices, China. Millipore MilliQ water with a resistivity of $18.2 \mathrm{~m} \Omega \cdot \mathrm{cm}^{-1}$ was used in all preparations. PC-12 cells (ATCC@ CRL-1721 ${ }^{\mathrm{TM}}$ ) F-12K Medium (Kaighn's Modification of Ham's F-12 Medium) (ATCC@ 30-2004 ${ }^{\mathrm{TM}}$ ) and Horse Serum (ATCC $® 30-2040^{\mathrm{TM}}$ ) were ordered from ATCC, Germany.

\subsection{PTMC-MA synthesis and characterization}

Trimethylene carbonate (TMC) and the initiator trimethylolpropane (TMP) were heated under an inert environment, which resulted in the ring opening polymerization towards three armed poly(trimethylene carbonate) (PTMC). Stannous octoate $\left(\mathrm{Sn}(\mathrm{Oct})_{2}\right)$ was used as a catalyst for the polymerization. Polymerization was performed at $130^{\circ} \mathrm{C}$ for 3 days. Functionalization with methacrylic anhydride (MA) was carried following methods described by Geven et al. [10]. TMC conversion and MA functionalization of PTMC chain ends were analyzed by proton nuclear magnetic resonance $\left({ }^{1} \mathrm{H}-\mathrm{NMR}\right)$. The polymer was precipitated in cold ethanol to remove traces of dichloromethane, triethylamine and unreacted MA and finally dried till a constant weight of the polymer was reached.

\section{3 rGO synthesis and characterization}

rGO was prepared with the top-down method in which graphite is oxidized, exfoliated and finally is reduced. The synthesis of GO was done following Hummer's method as described in by Tung et al. [11], except the replacement of $\mathrm{K}_{2} \mathrm{~S}_{2} \mathrm{O}_{8}$ for $\left(\mathrm{NH}_{4}\right)_{2} \mathrm{~S}_{2} \mathrm{O}_{8}$. GO was reduced using hydrazine monohydrate as a reducing agent, as described by Park et al. [12]. To determine if 
the oxidation and reduction steps of the rGO synthesis were successful, Fourier transformed infrared spectroscopy (FTIR) was performed using the ATR-FTIR apparatus on dried graphite, GO and rGO powders that were compressed with the machines force gauge. Complementary on the FTIR data, X-ray photoelectron spectroscopy (XPS) was carried out to quantify the atomic composition of the powders. Measurements were performed with an energy resolution of $0.2 \mathrm{eV}$. To verify successful exfoliation of multi-layered graphite oxide to GO, atomic force microscopy (AFM) was performed on GO.

\subsection{PTMC-MA/rGO composite fabrication}

rGO was dispersed in DMF by sonication of 2 hours in order to create an even distribution of the rGO sheets in the DMF. PTMC-MA was dissolved in rGO/DMF with a known concentration until the desired ratio between PTMC-MA and $\mathrm{rGO}$ was reached $(0,0.5,1,2$ and $4 \mathrm{wt} \%$ ). Precipitation in cold ethanol took place to remove the DMF solvent from the composite material. The PTMC-MA/rGO was dried under vacuum until no weight change was observed. The dried PTMC-MA/rGO was dissolved in chloroform to create a substance that could be cast with a casting knife. The photo-crosslinker Irgacure 2959 was added up to $2.5 \mathrm{wt} \%$ relative to PTMC-MA. The aimed thickness of the films was $100 \mu \mathrm{m}$ after chloroform evaporation. After casting, the films were covered and the chloroform evaporated overnight in a fume hood. The films were dried overnight and subsequently crosslinked in an $\mathrm{N}_{2}$ environment using an UVcrosslinker (Ultra-Lum Electronic Ultraviolet Crosslinker) at $365 \mathrm{~nm}$ and $11 \mathrm{~mW} \cdot \mathrm{cm}^{-2}$ for 30 minutes. The photo-crosslinked films were extracted in a series of Propylene carbonate/chloroform (volume ratio of 50/50 for one day, 75/25 for another day and the last day with pure ethanol) and subsequently dried in ambient conditions.

\subsection{Physical properties of PTMC-MA/rGO composites}

The degree of crosslinking of the PTMC-MA/rGO composites was determined by washing the films in chloroform for 7 days at room temperature. The chloroform was changed after 3 days. The degree of crosslinking was calculated using:

$$
\mathrm{G}=\frac{(1-\mathrm{x}) \mathrm{m}_{\mathrm{d}}}{(1-\mathrm{x}) \mathrm{m}_{\mathrm{i}}} \cdot 100 \%
$$


The weight fraction of the $\mathrm{rGO}$ in the PTMC-MA/rGO composite is $\mathrm{x}$, the mass of the films after extraction and drying is $\mathrm{m}_{\mathrm{d}}$ and the mass of the films prior to extraction is $\mathrm{m}_{\mathrm{i}}$. Thermogravimetric Analysis (TGA) performed on TGA 7 Perkin Elmer was executed on the temperature range of $30^{\circ} \mathrm{C}$ to $500^{\circ} \mathrm{C}$ with a heating and cooling rate of $20^{\circ} \mathrm{C} / \mathrm{min}$.

Water uptake by the PTMC-MA/rGO films was analysed by measuring the dry mass of the films and the mass after being submerged in Milli-Q water for 7 days at room temperature. The water uptake was defined using the following equation:

$$
\mathrm{WU}=\frac{\mathrm{m}_{\mathrm{w}}-\mathrm{m}_{\mathrm{d}}}{\mathrm{m}_{\mathrm{d}}} \cdot 100 \%
$$

The mass of the wet PTMC-MA/rGO films is $m_{w}$ and the mass of dry films is $m_{d}$.

The contact angle measurements were carried out with a volume of $2.7 \mu 1$ and imaged using a CCD camera and analysis software.

\subsection{Conductive properties of PTMC-MA/rGO composite films}

Van der Pauw (VDP) measurements were performed using a 4 probe measuring station. For this experiment, square samples $(25 \times 25 \mathrm{~mm})$ with uniform height were cut from the PTMC$\mathrm{MA} / \mathrm{rGO}$ films. The contact points of the probes were located as close to the corner of the sample as possible. A bias current was applied on the films by two of the probes. The other two probes measured a voltage difference. The resulting IV curves were used to determine the resistance of the film. Following the following equations (3-5) the conductivity of the material could be determined:

$$
\mathrm{e}^{-\pi \frac{\mathrm{R}_{\text {horizontal }}}{\mathrm{R}_{S}}}+\mathrm{e}^{-\pi \frac{\mathrm{R}_{\text {vertical }}}{\mathrm{R}_{S}}}=1
$$

With $\mathrm{R}_{\text {horizontal }}$ representing the average of two measurements in which the current applying probes were horizontally located from the voltage measuring probes and $\mathrm{R}_{\mathrm{vertical}}$ the two measurements in which they are vertically orientated from one another. The sheet resistance is represented by $R_{s}$. Following equation 3 , $R_{S}$ can be used to calculate the sample resistivity $(\rho)$ : 


$$
\rho=\mathrm{R}_{\mathrm{s}} \cdot \mathrm{t}
$$

With $\mathrm{t}$ as the sample thickness in $\mathrm{cm}$. The resistivity of the sample is inversely related to the conductivity $(\sigma)$ following equation 5 :

$$
\sigma=\rho^{-1}
$$

The conductivity values were given and reported in $\mathrm{S} \cdot \mathrm{cm}^{-1}$.

\subsection{Mechanical properties of PTMC-MA/rGO composite films}

To determine the E-modulus of the different compositions of PTMC-MA/rGO films the Zwick Z020 universal tensile tester was used to follow the standard ASTM D882. The grip-to-grip separation was set to $25 \mathrm{~mm}$ and the crosshead speed was $50 \mathrm{~mm} / \mathrm{min}$. The films were strained until failure was achieved. The E-modulus was calculated by fitting a trend line in the elastic region of the stress/strain curve between 1 and $3 \%$ strain.

\subsection{PC-12 cell culture on PTMC-MA/rGO composites}

PC12 cells were cultured in standard culture conditions of $37^{\circ} \mathrm{C}$ and $5 \% \mathrm{CO}_{2}$ using a $\mathrm{F}-12 \mathrm{~K}$ medium supplemented with 15\% Horse Serum (HS) and 2.5\% Fetal Bovine Serum (FBS), according to providers instructions. PTMC-MA/rGO films were sterilized by soaking the films in $70 \%$ Ethanol and evaporating the ethanol in a sterile environment. PC-12 cells were statically seeded by pipetting cell suspension on the films. Seeding density was calculated to be 12,500 cells/well. The samples underwent complete media change every 2-3 days. After 1 and 5 days the surfaces were lysed and cell pallets were analysed using the CyQUANT® cell proliferation kit according to manufacturer's guidelines. 


\section{Results and discussion}

\subsection{PTMC-MA characterization}

TMC conversion to PTMC was checked by comparing the TMC peak $-\mathrm{CO}-\mathrm{O}-\mathrm{CH}_{2}-\mathrm{CH}_{2}-$ $\mathrm{CH}_{2}-\mathrm{O}-$ at 4.46 ppm with the PTMC $-\mathrm{CO}-\mathrm{O}-\mathrm{CH}_{2}-\mathrm{CH}_{2}-\mathrm{CH}_{2}-\mathrm{O}-$ peak at 4.24 ppm on the NMR spectrum. The monomer conversion of approximately $99 \%$ was achieved. Next, the $\mathrm{CH}_{3}$ group of the initiator trimethylolpropane was compared to MA double bond proton peaks present at $5.57 \mathrm{ppm}$ and $6.11 \mathrm{ppm}$. An average of $91 \%$ of functionalized chain ends could be observed.

\section{2 rGO characterization}

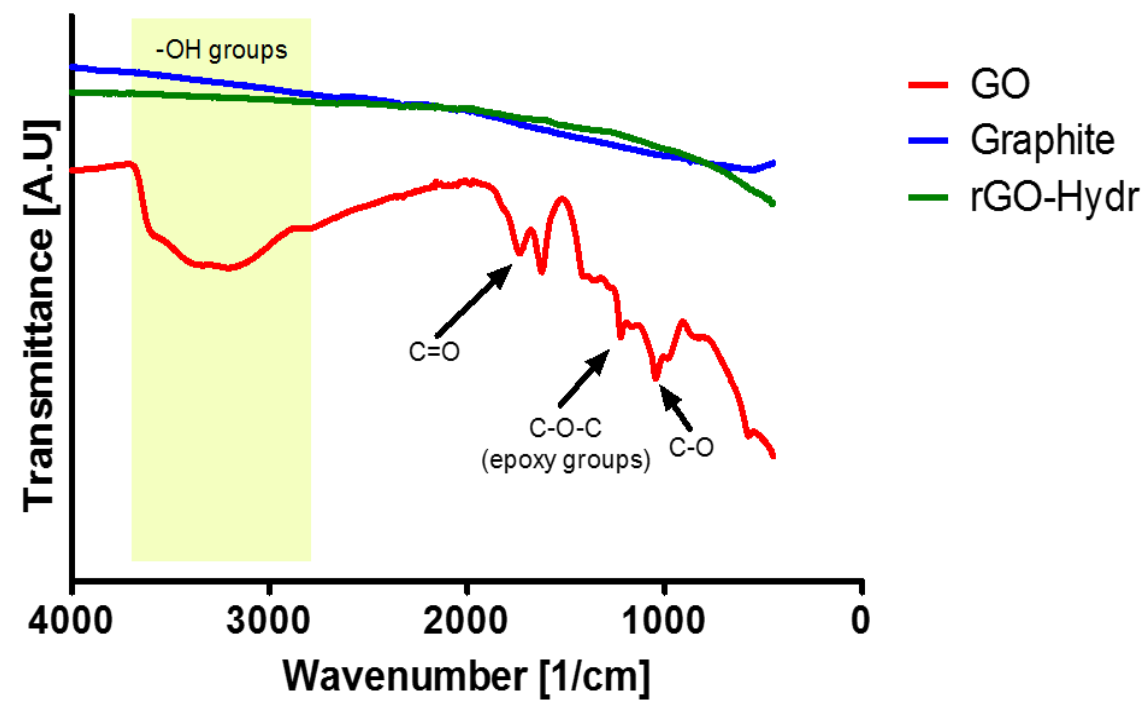

Figure 1 - Fourier transformed infrared (FTIR) spectrum of graphite, graphene oxide (GO) and reduced graphene oxide $(r G O)$. The characteristic peaks in the spectrum of $G O$ can be attributed to hydroxyl groups $\left( \pm 3700-2900 \mathrm{~cm}^{-1}\right)$, carboxyl groups $\left( \pm 1700 \mathrm{~cm}^{-1}\right)$, epoxygroups $\left( \pm 1200 \mathrm{~cm}^{-1}\right)$ and carbon-oxygen single bond $\left( \pm 1000 \mathrm{~cm}^{-1}\right)$. The lack of these peaks in the $r G O$ and its resemblance to graphite suggest a thorough removal of oxygen groups during the reduction by hydrazine monohydrate.

Figure 1 shows the FTIR spectrum is which the start material graphite, the intermediate product $\mathrm{GO}$ and the final product $\mathrm{rGO}$ were analysed. The presence of peaks in the GO spectrum that can be attributed to hydroxyl groups $\left( \pm 3700-2900 \mathrm{~cm}^{-1}\right.$, indicated with a yellow band), carboxyl groups $\left( \pm 1700 \mathrm{~cm}^{-1}\right)$, epoxy groups $\left( \pm 1200 \mathrm{~cm}^{-1}\right)$ and carbon-oxygen single bonds $\left( \pm 1000 \mathrm{~cm}^{-}\right.$ ${ }^{1}$ ) and indicate that oxidation by modified Hummers method was successful. The absence of 
these peaks in the rGO spectrum also support that the chemical reduction with hydrazine monohydrate was efficient.

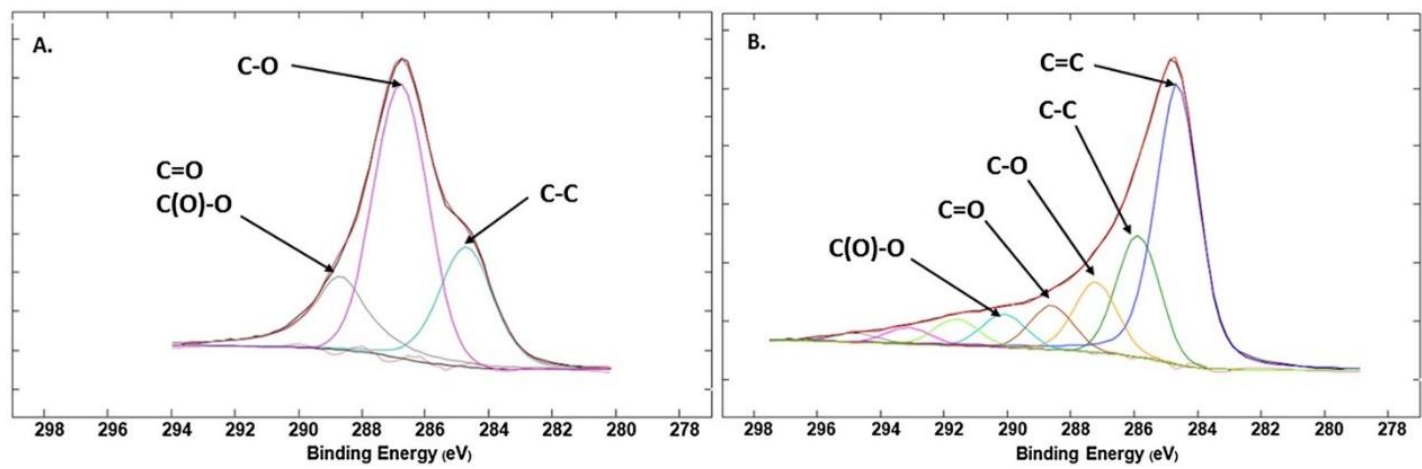

Figure 2 -XPS spectra of $G O(A)$ and $r G O(B)$. As a result of the reduction with hydrazine, the dominant $C-O$ peak in the spectrum of $G O$ is greatly diminished in the $r G O$ spectrum.

Table 1. XPS analyses performed on graphite, GO and $r G O$

\begin{tabular}{lcccc}
\hline Material & Atomic \% C & Atomic \% O & C : O ratio & Reference \\
\hline Graphite & $97.53 \pm 0.09$ & $2.47 \pm 0.09$ & 39.5 & This work \\
\hline GO & $59.10 \pm 0.18$ & $38.71 \pm 0.29$ & 1.5 & This work \\
\hline rGO & $89.62 \pm 0.24$ & $6.15 \pm 0.14$ & 13.9 & This work \\
\hline Dang et al. & - & - & 13.8 & {$[19]$}
\end{tabular}

Atomic percentages are derived from the XPS signals. The $\mathrm{C}: \mathrm{O}$ ratio of the GO shows that the modified Hummers' method performed in this work oxidized the graphite to a great extent. The subsequent reduction with hydrazine was also successful, increasing the $\mathrm{C}: \mathrm{O}$ ratio to 13.9 , only leaving approximately 6 atomic $\%$ of oxygen on the carbon sheet.

Subsequently, an XPS analysis was performed on dried graphite, GO and rGO powders. Spectra of GO and rGO are shown in Figure 2. The spectrum of GO reveals the presence of $\mathrm{C}-$ $\mathrm{O}$ bonds at $286.77 \mathrm{eV}$ and $\mathrm{C}=\mathrm{O}$ at $288.68 \mathrm{eV}$. The spectra of rGO show an increase of nonoxygenated carbon bonds and a drastic decrease of oxygen groups, indicating that reduction took place on the surface of the GO flakes. The $\mathrm{C}: \mathrm{O}$ ratio is often used in literature to describe the extent of oxidation of graphite and degree of GO reduction. Table 1 shows a summary of atomic composition and the $\mathrm{C}: \mathrm{O}$ ratio of the different materials. The $\mathrm{C}: \mathrm{O}$ ratio of 13.9 of the rGO indicates proper reduction of the oxygenated surface of the GO. 
AFM was performed after the exfoliation of GO into fewlayered GO structures, prior to the reduction reaction. Figure 3 shows an AFM image and the corresponding height profile. With lateral sizes of the GO flakes in the micron and sub-micron range and the height profile in the nanometer range, the aspect ratio of the GO particles is approximately 750 . This is in accordance with reports of aspect ratios of carbon fillers between 100 and 1000 [13]. High aspect ratios are keys when particles require a low percolation threshold when mixed in a polymer matrix [14].
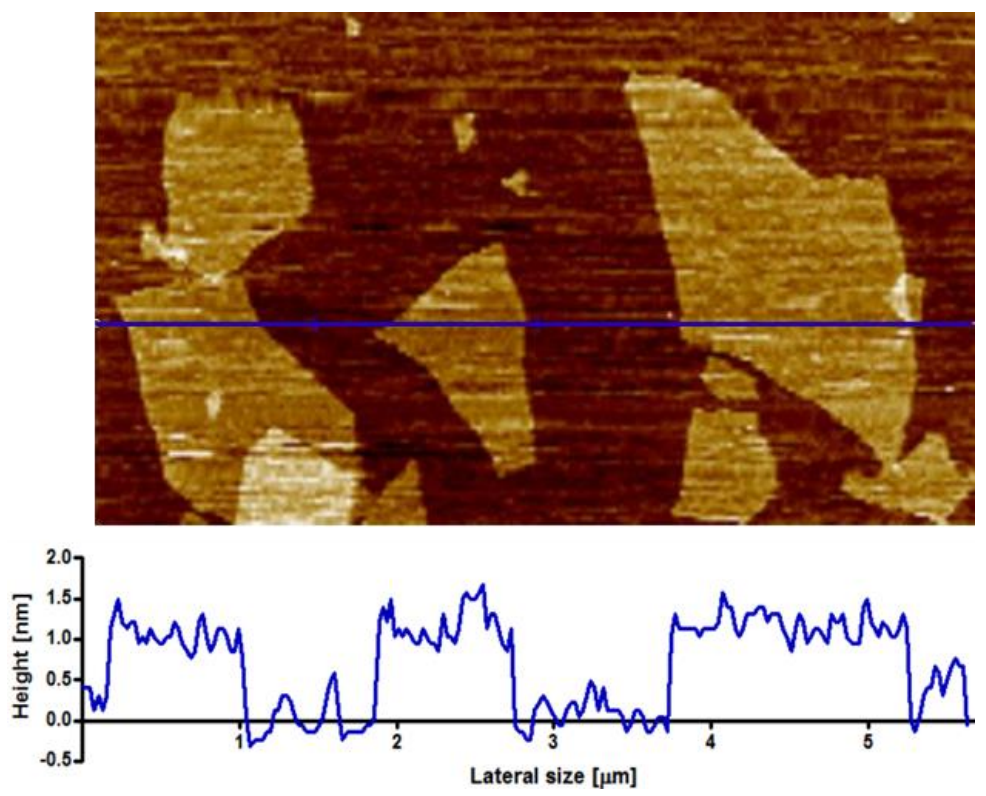

Figure 3 - Imaging of GO flakes by atomic force microscopy and the resulting height profile of GO on a freshly cleaved mica surface. The lateral size of the GO flakes was in the $\mu \mathrm{m}$ range with flakes with a size of approximately $1 \mu \mathrm{m}$. The height profile of these flakes was showed that the flakes were close to $1.5 \mathrm{~nm}$ thick. This would be equivalent to bi-layer or three layer structures.

\subsection{Physical properties of PTMC-MA/rGO composites}

Information about the degree of crosslinking, water uptake, and rGO loading of the PTMCMA/rGO composites can be found in Table 2. All measurements were performed in triplicates. The degree of crosslinking was higher than $88 \%$ for all composite materials, with an exception for $4 \mathrm{wt} \%$ rGO containing films. PTMC-MA/rGO with $4 \mathrm{wt} \%$ rGO loading only showed $17 \%$ gelation and thus failed to create a crosslinked network. It is hypothesized that the UV light could not reach the MA end groups due to shielding by the high amount of rGO particles present in the composite. This hypothesis is supported by UV-VIS spectra presented by Yang 
et al. [15] which show absorption of wavelengths in the UV-VIS range by rGO flakes in dispersion. These findings on gelation limits the amount of rGO that can be added to composite, when following the methods based on UV crosslinking.

Water uptake by the composite films did not differ significantly from earlier findings of pristine PTMC,[16] regardless of their rGO content. When examining the hydrophobicity of rGO and pristine PTMC, similar contact angles were observed on flat surfaces indicating similar interaction with aqueous solutions.[16,17] Low water uptake of the PTMC-MA/rGO films also suggests that the degree of swelling of the material is low, which is a beneficial characteristic for a material aimed to be used for the preparation of NGCs.

Table 2. Characteristics of PTMC-MA/rGO films with varying $r G O$ content

\begin{tabular}{lcc}
\hline Composite type & Degree of crosslinking, \% & Water uptake, wt\% \\
\hline $\mathbf{0}$ wt \% rGO & $92.02 \pm 3.60$ & $5.51 \pm 0.88$ \\
\hline $\mathbf{0 . 5}$ wt\% rGO & $89.35 \pm 1.33$ & $4.02 \pm 1.16$ \\
\hline $\mathbf{1}$ wt\% rGO & $89.38 \pm 1.10$ & $1.71 \pm 1.11$ \\
\hline $\mathbf{2}$ wt\% rGO & $88.09 \pm 1.58$ & $2.58 \pm 0.29$ \\
\hline $\mathbf{4}$ wt\% rGO & $17.32 \pm 1.83$ & $4.44 \pm 1.63$
\end{tabular}

PTMC-MA/rGO composites up to $2 \mathrm{wt} \% \mathrm{rGO}$ show excellent crosslinking with gel contents higher than $88 \%$. Increasing the rGO content to $4 \mathrm{wt} \%$ resulted in failure of network formation. The water uptake of the PTMC-MA/rGO films corresponded with earlier data from pristine PTMC-MA networks.[16] From these results, it can be concluded that increasing the rGO content of PTMC-MA/rGO composite films does not influence water uptake.

\subsection{Conductive properties of PTMC-MA/rGO composite films}

In order to characterize the conductive properties of the PTMCMA/rGO composites properly, an exact determination of the rGO content was needed, which can be found in Table 3. By means of TGA, it was confirmed that the 0,2 and $4 \mathrm{wt} \%$ composite films contained rGO amounts in agreement with the aimed value (within a 95\% confidence interval, two-tailed ttest). This was not the case for the 0.5 and $1 \mathrm{wt} \%$ composite films. These showed a significant 
Table 3 - Characteristics of PTMC-MA/rGO films with varying rGO content. TGA was used to determine the residual weight of the films at $500^{\circ} \mathrm{C}$. This weight relates to the amount of $r G O$ present in the composite.

\begin{tabular}{lccc}
\hline $\begin{array}{l}\text { Group (aimed } \\
\text { rGO loading) }\end{array}$ & $\begin{array}{c}\text { Residual weight at } \\
\mathbf{5 0 0}^{\circ} \mathbf{C}, \mathbf{w t} \%\end{array}$ & Conductivity, S/cm & Reference \\
\hline $\mathbf{0}$ wt\% rGO & $0.40 \pm 0.34$ & $5.99 \times 10^{-5}$ & This work \\
\hline $\mathbf{0 . 5}$ wt\% rGO & $1.16 \pm 0.10^{\mathrm{a}}$ & $5.91 \times 10^{-5}$ & This work \\
\hline $\mathbf{1}$ wt\% rGO & $1.67 \pm 0.18^{\mathrm{a}}$ & $1.55 \times 10^{-4}$ & This work \\
\hline $\mathbf{2}$ wt\% rGO & $2.16 \pm 0.54$ & $6.88 \times 10^{-2}$ & This work \\
\hline $\mathbf{4}$ wt\% rGO & $4.09 \pm 0.33$ & $7.74 \times 10^{-2}$ & This work \\
\hline Sayyar et al. & 10 & $\sim 10^{-2}$ & {$[18]$} \\
\hline
\end{tabular}

Incorporation of more than $0.5 \mathrm{wt} \% \mathrm{rGO}$ in the composite material resulted in conductive behavior. The percolation threshold of the rGO flakes produced in this study was determined to be between 1 and $2 \mathrm{wt} \%$ rGO content. Below $1 \mathrm{wt} \%$ rGO content, the conductivity of the composite was equal to that of pristine PTMC-MA and therefore too low to be measured precisely. At $2 \mathrm{wt} \% \mathrm{rGO}$ content, after the percolation threshold was reached, the measured conductivity was $6.88 \times 10^{-2} \mathrm{~S} \mathrm{~cm}^{-1}$. Increasing the rGO content to 4 $\mathrm{wt} \%$ did not change the conductive properties. ${ }^{*} \mathrm{P}<0.05$ as compared with the aimed rGO content, determined by a two-tailed t-test.

discrepancy with the aimed value of rGO loading. Uneven dispersions of rGO in the PTMCMA matrix might be contributing to these findings. However, the low SD values of residual weight determined by TGA indicate that there was a high uniformity between the triplicate samples. The conductive properties were assessed with the four-probe van der Pauw method. The conductivities of all PTMC-MA/rGO films are shown in Table 3. A rapid increase of conductive properties is expected after the percolation threshold of the rGO particles is reached. The percolation threshold of the rGO that was produced in this work was reached between 1 and $2 \mathrm{wt} \%$ rGO loading of the composite material. TGA analysis showed that the measured rGO concentrations of these groups were 1.67 and $2.16 \mathrm{wt} \%$ respectively. A conductivity of $6.88 \times 10^{-2} \mathrm{~S} \mathrm{~cm}^{-1}$ was measured for the films containing $2.16 \mathrm{wt} \% \mathrm{rGO}$. After the percolation threshold was reached, doubling the amount of rGO filler in the composite to approximately 4 wt\% did not result in a higher conductivity. Sayyar et al. prepared composites consisting of polycaprolactone and rGO and also measured conductivity values in the range of $10^{-2} \mathrm{~S} \mathrm{~cm}^{-1}$, by adding $10 \mathrm{wt} \%$ of rGO in their composites [18]. 


\subsection{PC-12 cell culture on PTMC-MA/rGO composites}

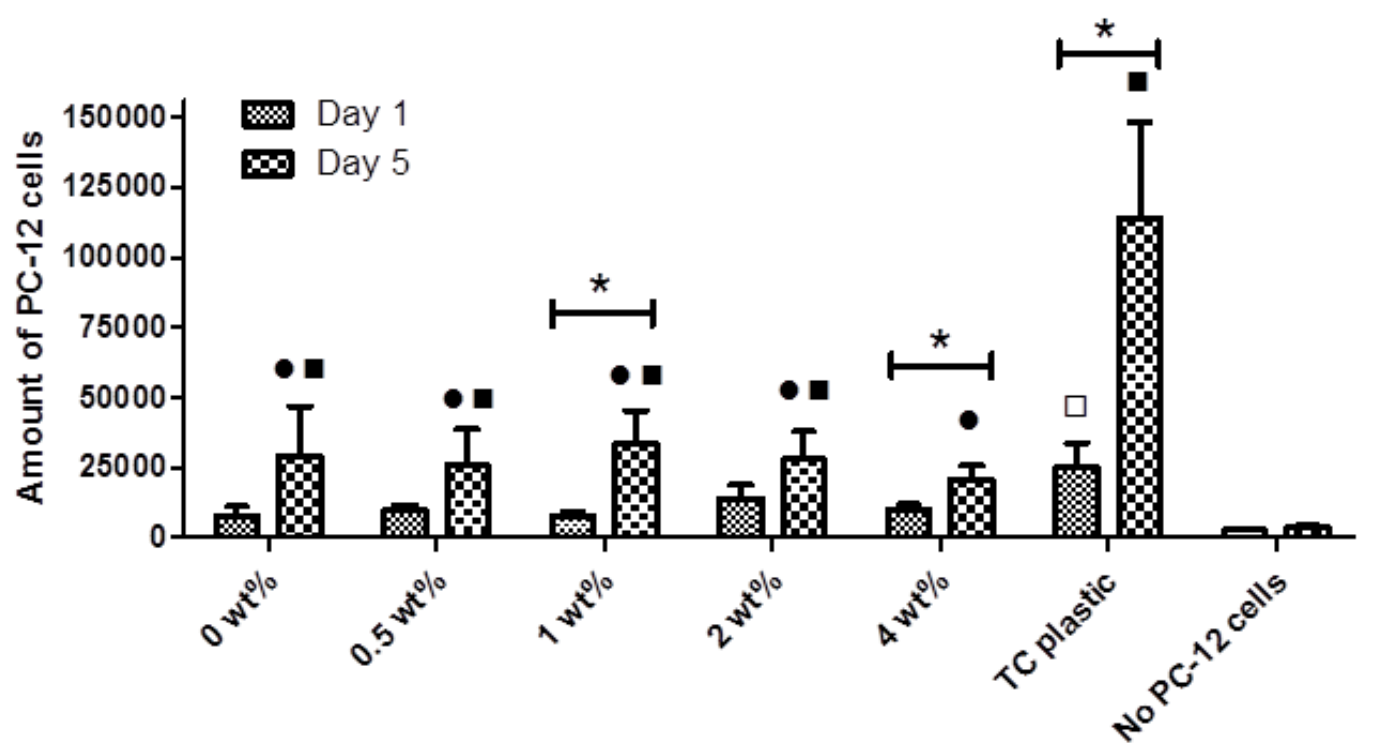

Figure $4-P C-12$ cell quantification by CyQUANT® cell proliferation assay kit on PTMC-MA/rGO films. All cell counts were significantly different from films without PC-12 cells after 5 days of incubation at $37^{\circ} \mathrm{C}$ and $5 \% \mathrm{CO}_{2}$, except the culture on PTMC-MA/RGO with 4 wt\% rGO incorporated. This indicates that cells remain viable on the composite films. The degree of cell proliferation could be analysed when comparing the results after 1 and 5 days of culturing. Films with 1 wt\% and 4 wt\% showed significant difference and thus provided statistical evidence of cell proliferation. Statistics were conducted using 2-way ANOVA.

$\square=P<0.05$ relative to blank films at day $1 ; \mathbf{\square}=P<0.05$ relative to blank films at day $5 ; \bullet=P<0.05$ relative to tissue culture (TC) plastic at day $5 . *=P<0.05$ between day 1 and day 5 cultures.

The cell quantification by CyQUANT® cell proliferation assay kit of PC-12 cultures on PTMC-MA/rGO films with varying rGO content can be seen in Figure. 4. All cell counts were significantly different from films without PC-12 cells after 5 days of incubation at $37^{\circ} \mathrm{C}$ and 5\% CO2, except the culture on PTMC-MA/rGO with 4 wt\% rGO content. Films with 1 and 4 wt $\%$ of rGO showed a significant difference in cell number between day 1 and day 5, indicating cell proliferation. Although cell quantifications of cultures on PTMC-MA/rGO films with 0, 0.5 and $2 \mathrm{wt} \%$ rGO also suggested proliferation of PC-12 cells, these increases were not statistically significant. Most importantly, the cell quantifications did not differ significantly between PTMC-MA/rGO films with varying rGO content. This indicates that the adhesion and proliferation of PC-12 cells are not affected by the presence of rGO within the range of 0 to 4 $\mathrm{wt} \%$. This is in agreement with earlier work on polycaprolactone/rGO composites by Sayyar et al [18]. The significant difference between all cultures on PTMC-MA/rGO composites with 
tissue culture plastic was unexpected, as literature reports behavior of PTMC-MA networks that is similar to tissue culture plastic [6]. The use of protein coatings like fibronectin have shown to provide better cell adhesion and proliferation and might be a next step in the development of PTMC-MA/rGO composites as biomaterial for the preparation of NGCs.

\section{Conclusion}

In this paper we presented a method for the synthesis of functionalized poly(trimethylene carbonate) and reduced graphene oxide composites. The conductive filler rGO could be synthesized by a multistep oxidation followed by exfoliation by ultrasound and reduction with hydrazine monohydrate. Stable composite networks with up to $2 \mathrm{wt} \%$ rGO content could be fabricated using solution mixing in dimethyl formamide and subsequent UV crosslinking. The materials showed an increase in conductive properties after the percolation threshold was passed with the addition of $2 \mathrm{wt} \% \mathrm{rGO}$, and conductivity values of $6.88 \cdot 10^{-2} \mathrm{~S} \cdot \mathrm{cm}^{-1}$ were measured. The PTMC-MA/rGO films proved biocompatible as adhesion and proliferation of PC-12 cells was shown by CyQUANT® cell proliferation assay kit. These results provide evidence that composite materials of PTMC and $\mathrm{rGO}$ have promising properties for an application for NGC's to accelerate nerve regeneration.

\section{Acknowledgements}

The authors would like to thank Gerard Kip associated with the MESA+ institute for measuring and analysing the XPS data.

\section{References}

[1] M.F. Meek, J.H. Coert, Clinical use of nerve conduits in peripheral-nerve repair: review of the literature, J. Reconstr. Microsurg., 18 (2002) 97-109.

[2] A. Faroni, S.A. Mobasseri, P.J. Kingham, AJ. Reid, Peripheral nerve regeneration: experimental strategies and future perspectives, Adv Drug Deliv Rev., 82 (2015) 160-167. 
[3] T. Gordon, O. A. R. Sulaiman, A. Ladak, Chapter 24 electrical stimulation for improving nerve regeneration: where do we stand? In: International Review of Neurobiology. Academic Press, 2009, 433-444.

[3] Gordon, T., O.A.R. Sulaiman, A. Ladak, Chapter 24 Electrical Stimulation for Improving Nerve Regeneration: Where do we Stand? in International Review of Neurobiology, (2009) 433-444.

[4] R. Balint, N.J. Cassidy, S.H. Cartmell, Electrical stimulation: a novel tool for tissue engineering, Tissue Eng. Part B Rev., 19 (2013) 48-57.

[5] A.P. Pego, A.A. Poot, D.W. Grijpma, J. Feijen, Biodegradable elastomeric scaffolds for soft tissue engineering, J. Control. Release, 87 (2003) 69-79.

[6] A.P. Pego, A.A. Poot, D.W. Grijpma, J. Feijen, Copolymers of trimethylene carbonate and epsilon-caprolactone for porous nerve guides: synthesis and properties, J. Biomater. Sci. Polym. Ed., 12 (2001) 35-53.

[7] E. Bat, J.A. Plantinga, M.C. Harmsen, M.J.A. van Luyn, Z. Zhang, D.W. Grijpma, J. Feijen, Trimethylene carbonate and epsilon-caprolactone based (co)polymer networks: mechanical properties and enzymatic degradation, Biomacromolecules, 9 (2008) 3208-3215.

[8] E. Bat, J.A. Plantinga, M.C. Harmsen, M.J.A. Van Luyn, J. Feijen, D.W. Grijpma, Ultraviolet light crosslinking of poly (trimethylene carbonate) for elastomeric tissue engineering scaffolds, Biomaterials, 31 (2010) 8696-8705.

[9] V.H. Pham, T.T. Dang, S.H. Hur, E. J. Kim, J.S. Chung, Highly Conductive poly(methyl methacrylate) (PMMA)-reduced graphene oxide composite prepared by self-assembly of PMMA latex and graphene oxide through electrostatic interaction, ACS Appl. Mater. Interfaces, 4 (2012) 2630-2636.

[10] M.A. Geven, D. Barbieri, H. Yuan, J.D. de Bruijn, D.W. Grijpma, Preparation and mechanical properties of photo-crosslinked poly(trimethylene carbonate) and nanohydroxyapatite composites, Clin. Hemorheol. Microcirc., 60 (2015) 3-11.

[11] V.C. Tung, M.J. Allen, Y. Yang, R.B. Kaner, High-throughput solution processing of large-scale graphene, Nat. Nano, 4 (2009) 25-29. 
[12] S. Park, J.A. Jung, R.D. Piner, S.J. An, X. Li, A. Velamakanni, R.S. Ruoff, Colloidal suspensions of highly reduced graphene oxide in a wide variety of organic solvents, Nano Lett., 9 (2009) 1593-1597.

[13] G. Kaur, R. Adhikari, P. Cass, M. Bown, P. Gunatillake, Electrically conductive polymers and composites for biomedical applications, RSC Adv., 5 (2015) 37553-37567.

[14] P.N. Khanam, D. Ponnamma, M.A. AL-Madeed, Electrical properties of graphene polymer nanocomposites. In: graphene-based polymer nanocomposites in electronics, Springer International Publishing: Cham., (2015) 25-47.

[15] S. Yang, W. Yue, D. Huang, C. Chen, H. Lin, Z. Yang, A facile green strategy for rapid reduction of graphene oxide by metallic zinc, RSC Adv., 2 (2012) 8827-8832.

[16] Z. Zhang, R. Kuijer, S.K. Bulstra, D.W. Grijpma, J. Feijen, The in vivo and in vitro degradation behavior of poly(trimethylene carbonate), Biomaterials, 27 (2006) 1741-1748.

[17] J.S. Lee, J.C. Yoon, J.H. Jang, A route towards superhydrophobic graphene surfaces: surface-treated reduced graphene oxide spheres, J. Mater. Chem. A, 1 (2013) 7312-7315.

[18] S. Sayyar, E. Murray, B.C. Thompson, S. Gambhir, D.L. Officer, G.G. Wallace, Covalently linked biocompatible graphene/polycaprolactone composites for tissue engineering, Carbon, 52 (2013) 296-304.

[19] T.T. Dang, V.H. Pham, S.H. Hur, E.J. Kim, B.S. Kong, J.S. Chung, Superior dispersion of highly reduced graphene oxide in N, N-dimethylformamide, J. Colloid Interface Sci., 376 (2012) 91-96. 


\section{Chapter 4 - Evaluation of a PTMC/rGO composite nerve guide conduit: An in vivo pilot study}

Zhengchao Guo, Dirk W. Grijpma, André A. Poot

Department of Biomaterials Science and Technology, University of Twente, Enschede, The Netherlands. 


\begin{abstract}
The use of electrically conductive materials for the fabrication of nerve guide conduits has been shown to be a viable strategy to improve peripheral nerve regeneration. In the present study, a porous nerve guide based on a composite of poly(trimethylene carbonate) (PTMC) and reduced graphene oxide (rGO) with $2 \mathrm{wt} \%$ rGO relative to PTMC was prepared by dip-coating, photocrosslinking and leaching of $\mathrm{NaF}$ porogen particles. The conduit, which had a porosity of $73 \%$, was used to bridge a $15 \mathrm{~mm}$ sciatic nerve gap in a pilot study in the rabbit. A porous PTMC conduit with the same porosity was used as a control. Macroscopic evaluation 6 weeks after implantation showed the formation of firm tissue in the PTMC/rGO nerve guide, whereas the tissue formed in the PTMC conduit lacked form stability. Histological analysis showed that the number of myelinated axons and the amount of collagen were higher in the PTMC/rGO nerve guide than in the PTMC conduit. The same differences were found in the respective distal nerve stumps. It is concluded that PTMC/rGO composite is an interesting material for the fabrication of conduits for peripheral nerve regeneration.
\end{abstract}

\title{
1 Introduction
}

The repair of peripheral nerve injuries is a remaining clinical problem, which requires a lot of efforts from both materials and biological sciences in order to be solved [1]. Autologous nerve grafts are considered as the gold standard to bridge peripheral nerve defects. However, this requires extra surgery and results in donor site morbidity. Therefore, a strategy for axonal regeneration was developed, based on the use of a nerve guide conduit to bridge the proximal and distal nerve stumps at the site of a defect [2]. Up to now, however, the recovery of nerve function by implantation of commercially available nerve guides is still not as good as obtained with autologous nerve grafts [3].

Electrically conductive materials, including conductive polymers and composites of polymers with conductive fillers, are widely used in biomedical applications such as bone and neural tissue engineering $[4,5]$. Conducting polymers such as polypyrrole (PPY), polyaniline (PAN), polythiophene (PTH) and poly(3,4-ethylene dioxythiophene) (PEDOT) are regarded as good candidates to improve neuronal cell function [6]. However, these polymers are mechanically brittle, affecting the suturability of conduits prepared from them. In addition, their application is limited due to long-term toxicity [7]. Regarding composites of polymers with conductive 
fillers, gold and silver particles, carbon nanotubes and graphene nanosheets have been selected because of their high electrical conductivity and biocompatibility [8] [9] [10] [11]. As a type of graphene, reduced graphene oxide (rGO) has been successfully used for this application. Graphene oxide (GO) is first synthesized from graphite and subsequently thermally or chemically reduced to rGO. In this way, relatively large amounts of rGO can be obtained at low cost [12]. Several polymer/rGO composites were prepared, which showed both good electrical conductivity and biocompatibility in vitro [13][14][15]. Moreover, enhanced neuronal cell differentiation and signaling were observed on a polymer/rGO composite substrate in vitro [16]. However, in vivo nerve regeneration studies using rGO-based materials have hardly been reported. As recently shown by Wang et al., an rGO-coated electrospun fibrous nerve guide performed equally well as autologous nerve graft in a rat sciatic nerve gap model [17]. Hence, polymer/rGO composite nerve guides are expected to show good results in vivo as well.

A functional nerve guide conduit should match several physical properties such as porosity, flexibility, a low degree of swelling and a proper degradation rate [18]. Using a polymer-based composite, the mechanical and swelling properties as well as degradation rate are mainly determined by the polymer matrix. Poly(trimethylene carbonate) (PTMC) is an amorphous polymer that is frequently used for soft tissue engineering [19]. Scaffolds prepared from crosslinked PTMC networks are flexible and elastic. Water uptake by hydrophobic PTMC is low, resulting in a low degree of swelling. The degradation rate can be tuned by varying the crosslink density [20] [21]. In contrast to e.g. poly(caprolactone) (PCL) and poly(lactic acid) (PLA), PTMC degrades by a surface erosion mechanism without the formation of acidic degradation products [22]. One of the main functions of a nerve guide is to protect the regenerating axons from invading cells from the environment, i.e. prevention of scar tissue formation. The wall of the conduit, however, should not be completely closed in order to sustain the delivery of nutrients and removal of cellular waste products. Particle leaching is an efficient way to make a porous structure, as previously reported by us concerning the preparation of porous tubular PTMC scaffolds for vascular tissue engineering [23].

In a previous study, we prepared electrically conductive PTMC/rGO composite films by mixing an rGO dispersion with a PTMC solution. Photo-crosslinked PTMC/rGO films showed good biocompatibility with PC12 rat neuronal cells [24]. In the present study, a porous nerve guide conduit based on a PTMC/rGO composite was prepared by combined dip coating, photocrosslinking and particle leaching. Subsequently, the conduit was applied in a pilot study on nerve regeneration in a rabbit sciatic nerve gap model, using a porous PTMC conduit as a control. 


\section{Materials and methods}

\subsection{Materials}

Trimethylene carbonate (TMC) was kindly provided by Huizhou Foryou Medical Devices Co., China. 1,1,1-Tris(hydroxymethyl)propane, stannous octoate, methacrylic anhydride, triethylamine, hydroquinone, Irgacure 2959, methylene blue and basic fuchsin were ordered from Sigma Aldrich, Netherlands. Dimethylformamide (DMF), dichloromethane (DCM), ethanol, chloroform and $\mathrm{NaF}$ particles (size range 1-106 $\mu \mathrm{m}$ ) were purchased from VWR chemicals, Netherlands. Propylene carbonate (PC), sodium pentobarbital, buprenorphine and penicillin were obtained from Merck, Netherlands.

\subsection{Preparation and characterization of photo-crosslinkable PTMC macromer, rGO and PTMC/rGO composite}

Three-armed PTMC was synthesized by ring-opening polymerization of TMC under argon atmosphere at $130{ }^{\circ} \mathrm{C}$ for 3 days [24]. 1,1,1-Tris(hydroxymethyl)propane and stannous octoate were used as initiator and catalyst, respectively. After dissolving the obtained PTMC in DCM, hydroquinone, triethylamine and methacrylic anhydride were added, and the mixture was reacted under argon protection at room temperature (RT) for 5 days in the dark [24]. Photocrosslinkable PTMC macromer was obtained by precipitation in cold ethanol and drying under vacuum at $\mathrm{RT}$ in the dark until constant weight. The molecular weight $\left(\mathrm{M}_{\mathrm{n}}\right)$ and degree of functionalization of the PTMC macromer were determined by ${ }^{1} \mathrm{H}-\mathrm{NMR}$ spectroscopy using a Bruker Ascend 400/Avance III 400 MHz NMR spectrometer.

rGO was prepared as previously described [24]. Briefly, GO was synthesized by a modified Hummers' method and reduced to rGO using hydrazine monohydrate [25].

PTMC macromer was dissolved in chloroform and $2 \mathrm{wt} \%$ rGO relative to PTMC was dispersed in DMF by sonication for $2 \mathrm{~h}$ in an ice bath. After mixing the PTMC solution and the rGO suspension at RT for $4 \mathrm{~h}$, the mixed dispersion was precipitated in cold ethanol and the obtained PTMC/rGO composite was dried under vacuum at RT in the dark until constant weight. 


\subsection{Fabrication and characterization of PTMC and PTMC/rGO nerve guide conduits}

The nerve guide conduits were fabricated by dip-coating using a $3 \mathrm{~mm}$ diameter glass mandrel. In detail, 1g PTMC macromer or PTMC/rGO composite was dissolved in $10 \mathrm{ml}$ chloroform and $4.56 \mathrm{~g} \mathrm{NaF}$ was added as a porogen, resulting in a theoretical porosity of $70 \%$. After adding Irgacure 2959 (2 wt\% relative to PTMC macromer) as a photo-initiator, the mixed dispersion in chloroform was transferred to a glass test tube. The mandrel was dip-coated 8 times into the dispersion to reach a layer thickness of around $1.5 \mathrm{~mm}$. After each dip-coating, the mandrel was dried slowly by horizontal rotation for $5 \mathrm{~min}$. In this way, a uniform layer thickness along the glass mandrel was obtained. After drying overnight in the dark to evaporate chloroform, the mandrel with polymer matrix was transferred to a UV cabinet and exposed under nitrogen flow for $1 \mathrm{~h}$ to light with a wavelength of $365 \mathrm{~nm}$ and an intensity of $8 \mathrm{~mW} / \mathrm{cm}^{2}$. To ensure homogeneous crosslinking, the mandrel was turned 180 degrees after $30 \mathrm{~min}$. A solution of 30 $\mathrm{ml}$ chloroform and $70 \mathrm{ml} \mathrm{PC}$ was used to swell and detach the tubular structure from the mandrel. Moreover, the sol fraction was extracted in this solution, which was refreshed once. Subsequently, the tubular structure was immersed in ethanol which was refreshed several times, after which the structure was dried in air overnight. Finally, the NaF particles were leached in distilled water which was refreshed several times, after which the porous conduit was dried in air.

The porosity of the PTMC and PTMC/rGO nerve guide conduits was calculated by the following equation,

$$
\text { Porosity }=\left[1-\frac{m_{d r y}}{V_{d r y} \times \rho_{P T M C}}\right] \times 100 \%
$$

in which $\rho_{\mathrm{PTMC}}=1.31 \mathrm{~g} / \mathrm{cm}^{3}$ (the density of the PTMC $/ \mathrm{rGO}$ composite was assumed to be the same), $\mathrm{m}_{\text {dry }}$ is the dry weight of the sample and $\mathrm{V}_{\text {dry }}$ the sample's bulk volume.

The structure of the nerve guides was observed by scanning electron microscopy (SEM) using a JEOL JSM-IT100 microscope. Before observation, samples were coated with gold in a Cressington sputter coater 108 Auto set at $40 \mathrm{~mA}$ for $60 \mathrm{~s}$.

Water flux measurements were conducted using PTMC films with $70 \%$ porosity to reflect water permeance of the PTMC and PTMC/rGO nerve guide conduits. The porous PTMC films were 
prepared in a similar way as described for the conduits. Instead of dip-coating, a PTMC macromer, Irgacure 2959 and NaF mixture in chloroform was cast on a glass plate. The photocrosslinked porous PTMC films had a thickness of around $0.4 \mathrm{~mm}$. Circular pieces with a diameter of $26 \mathrm{~mm}$ were punched from the films and sealed in an Amicon cell 8003 with 0.9 $\mathrm{cm}^{2}$ filtration area (Merck Millipore). MilliQ water was introduced to the films at different pressures. After obtaining a stable water flow, the weight of the permeating water was measured every $10 \mathrm{~s}$ for a minimum of $20 \mathrm{~min}$.

\subsection{Implantation of PTMC and PTMC/rGO nerve guide conduits}

Two New Zealand white rabbits (female, 6 months, 3.5-4.5 kg) were used in this study with permission of the local ethical committee (Management Committee of Experimental Animals of Sichuan Province, China). Operations were carried out under general sterile conditions. All surgeries were conducted under general anesthesia by injection of sodium pentobarbital (30 $\mathrm{mg} / \mathrm{kg}$ body weight) in the marginal ear vein of the rabbits. Following skin incision, fascia and muscle groups were separated using blunt dissection, and a $15 \mathrm{~mm}$ long gap was created in the right sciatic nerve. Ethanol-disinfected PTMC or PTMC/rGO nerve guide conduits with a length of $20 \mathrm{~mm}$ were implanted to bridge the proximal and distal nerve segments, using a single 7-0 nylon suture at each side. The muscle layer was re-approximated with 4-0 chromic gut sutures, and the skin was closed with 4-0 silk sutures.

After surgery, the animals were intramuscularly given $0.1 \mathrm{mg}$ buprenorphine for 2 days to relieve pain and penicillin $(40 \mathrm{mg} / \mathrm{kg}$ ) for 3 consecutive days to prevent infection. The animals were fed ad libitum in separate stainless steel cages, and were allowed to move inside their cages without restriction. They were kept at $20-25{ }^{\circ} \mathrm{C}$ and $50-60 \%$ humidity, experiencing a light cycle coinciding with daylight hours.

\subsection{Histological analysis}

After 6 weeks, the rabbits were sacrificed with a marginal ear vein injection of an excessive amount of sodium pentobarbital, and samples were collected for histological evaluation. Firstly, the explanted nerve grafts were fixed in $10 \%$ formalin. Then, the conduits were opened, taking care not to harm the regenerated tissue inside, which was dehydrated in a series of ethanol solutions and embedded in methyl methacrylate (MMA). After curing at $60{ }^{\circ} \mathrm{C}$, the embedded 
samples were trimmed and mounted on a diamond saw microtome (Leica SP-1600, Wetzlar, Germany). Sections of $20 \mu \mathrm{m}$ were obtained and stained with $1 \%$ methylene blue and $0.3 \%$ basic fuchsin for histological and morphometrical evaluations. This method was used in the past to stain peripheral nerve tissue [26]. A similar method based on toluidine blue and alkaline fuchsin was used by den Dunnen et al. in an in vivo study of peripheral nerve regeneration [27].

Regenerated nerve tissue was examined in the middle parts of the PTMC and PTMC/rGO nerve guide conduits. Moreover, samples were taken from the distal nerve stumps $5 \mathrm{~mm}$ outside the conduits. Samples from the non-operated sciatic nerve of each animal were taken as controls. From each section, 4 areas of $200 \times 200 \mu \mathrm{m}$ were evaluated and the number of myelinated axons per area was quantified. A similar method was used by den Dunnen and Meek [28][29].

\subsection{Statistical analysis}

Differences in the number of myelinated axons were evaluated by two-way ANOVA using GraphPad Prism. $\mathrm{P}<0.05$ was considered statistically significant.

\section{Results and discussion}

\subsection{Characterization of PTMC and PTMC/rGO nerve guide conduits}

The synthesized PTMC macromer had a $\mathrm{M}_{\mathrm{n}}$ of $19 \mathrm{~kg} / \mathrm{mol}$ and a degree of functionalization with methacrylate groups of $97 \%$. Moreover, rGO was synthesized with a C:O ratio of 13.9. As described in our previous study [24], the conductivities of PTMC and PTMC/rGO composite with $2 \mathrm{wt} \%$ rGO were $5.99 \times 10^{-3} \mathrm{~S} / \mathrm{m}$ and $6.88 \mathrm{~S} / \mathrm{m}$, respectively.

Macroscopic images of the PTMC and PTMC/rGO conduits are shown in Figures $1 \mathrm{~A}$ and B, and SEM images of the respective cross-sections in Figures $1 \mathrm{C}$ and D. The presence of rGO is depicted by the black color of the composite nerve guide. The walls of both conduits showed a similar interconnected pore structure.

As shown in Table 1, the inner diameter and wall thickness of both conduits were around $3 \mathrm{~mm}$ and $0.4 \mathrm{~mm}$, respectively. Both porosity and pore size range were in line with the theoretical values of $70 \%$ and 1-106 $\mu \mathrm{m}$, respectively. Because of the alternating presence of large and 
small pores (Figure 1), cellular infiltration through the walls of the conduits was anticipated to be unlikely. The aim of the porous walls was to facilitate the transport of nutrients and cellular
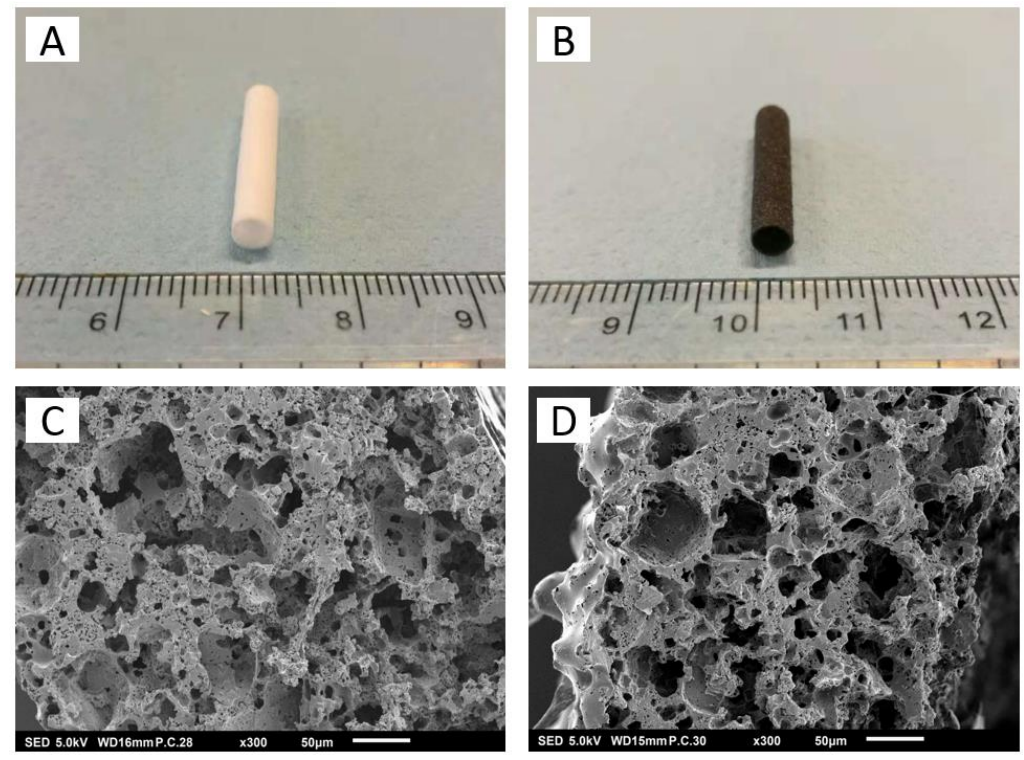

Figure 1. Macroscopic and SEM images of PTMC $(A, C)$ and PTMC/rGO $(B, D)$ nerve guide conduits. The SEM images show cross-sections of the walls of the conduits. Scale bars in C and D are $50 \mu m$.

waste products. This might be hampered by a hydrophobic nature of the polymer matrix [30]. However, a high water flux of $36,650 \pm 1,280 \mathrm{~L} / \mathrm{m}^{2} . \mathrm{h}$ at 0.1 bar was measured through porous PTMC films prepared in the same way. For comparison, Bettahalli et al. prepared porous PLA fibers with a water flux of $200 \mathrm{~L} / \mathrm{m}^{2} . \mathrm{h}$ at 0.1 bar, which supported the growth of mouse premyoblasts [31]. Hence, the tissue fluid permeability of the nerve guide conduits in the present study was considered to be sufficient for the intended application.

Table 1. Parameters of the PTMC and PTMC/rGO nerve guide conduits.

\begin{tabular}{lcc}
\hline & PTMC & PTMC/rGO \\
\hline Inner diameter, $\mathbf{m m}^{*}$ & $2.95 \pm 0.11$ & $3.02 \pm 0.13$ \\
\hline Wall thickness, $\mathbf{~ m m}^{*}$ & $0.43 \pm 0.07$ & $0.36 \pm 0.05$ \\
\hline Porosity, \%* & $71 \pm 1$ & $73 \pm 1$ \\
\hline Pore size range, $\boldsymbol{\mu m}$ & $1-103$ & $1-96$
\end{tabular}

$* \mathrm{~N}=4$. 


\subsection{Implantation of PTMC and PTMC/rGO nerve guide conduits}

As shown in Figures $2 \mathrm{~A}$ and $\mathrm{B}$, the PTMC and PTMC/rGO conduits were successfully implanted in the rabbits without any difficulties. The nerve guides were properly sutured at the defect site by two stitches. Upon sacrifice of the animals after 6 weeks, the conduits were still in place at the defect site and no polymer and/or rGO was found to be present in the surrounding tissue, see Figures $2 \mathrm{C}$ and D. After opening of the nerve guides, shown in Figures $2 \mathrm{E}$ and F, different types of tissue were found. The tissue in the PTMC guide lacked form stability and was sagging downward, whereas the tissue in the PTMC/rGO guide was more firm and had a tubular appearance resembling a nerve. In contrast to the PTMC conduit, the PTMC/rGO conduit fragmented upon opening. This can be explained by the presence of rGO, diminishing
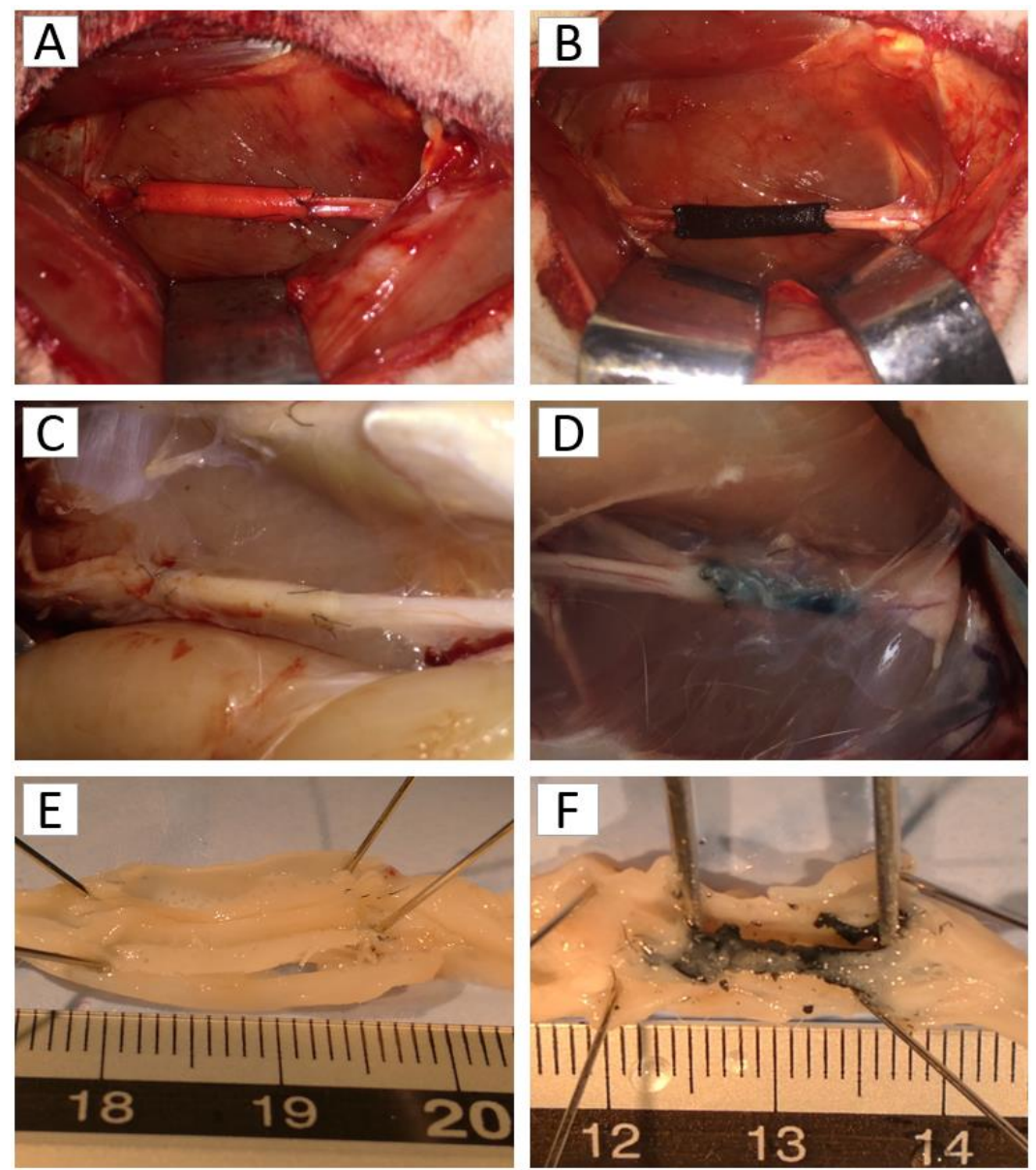

Figure 2. Macroscopic images of PTMC and PTMC/rGO nerve guide conduits at implantation (A and $B$, resp.), 6 weeks after implantation ( $C$ and $D$, resp.), and after explantation and opening of the conduits (E and $F$, resp.). 
UV light penetration during crosslinking, resulting in a lower crosslink density of the PTMC/rGO nerve guide as compared to the PTMC conduit. In future work, the crosslink density of PTMC/rGO nerve guides could be increased by using PTMC macromer with a lower molecular weight, resulting in a lower degradation rate [20].

\subsection{Histological analysis}

The tissues inside the PTMC and PTMC/rGO conduits were stained with methylene blue and basic fuchsin, showing non-myelinated axons in pale blue, myelinated axons in deep blue and collagen in red [26].

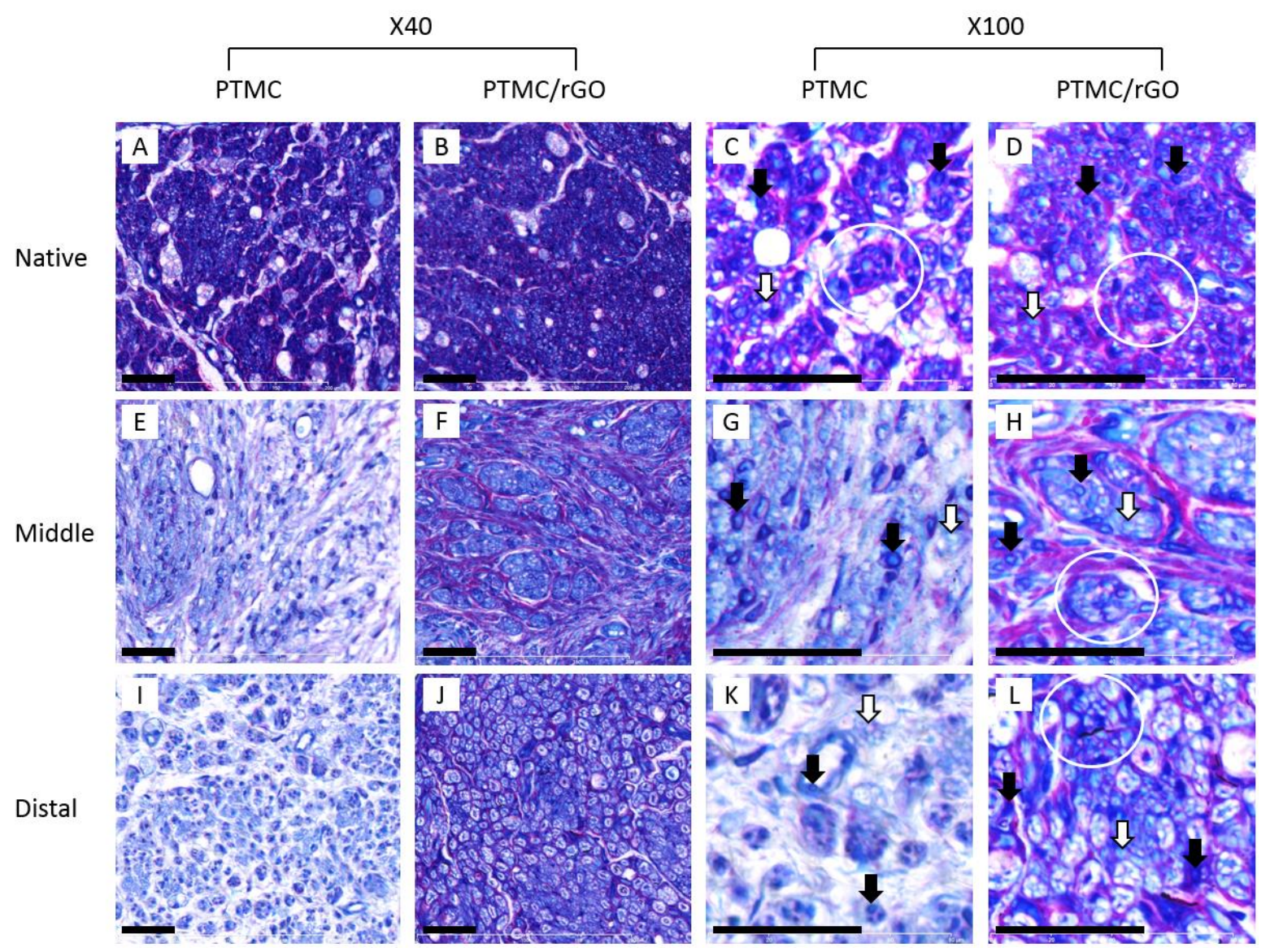

Figure 3. Histological images of cross-sections of native sciatic nerve of the rabbit $(A-D)$, regenerated tissue in the middle of the PTMC $(E, G)$ and PTMC/rGO $(F, H)$ nerve guide conduits, and the respective distal nerve stumps (I,K and $J, L)$. Non-myelinated axons: pale blue (white arrows), myelinated axons: deep blue (black arrows), collagen: red. Encircled in white: clusters of (non-)myelinated axons surrounded by collagen. Scale bars are $50 \mu \mathrm{m}$. 
Figure 3 shows cross-sections of the native sciatic nerves, regenerated tissue in the middle of the conduits, and the distal nerve stumps. The native sciatic nerves (Figures 3A-D) were similar, with plenty of myelinated axons, also non-myelinated axons and collagen. Large differences were found between the tissues present in the middle of the nerve guides, as more myelinated axons and collagen were present in the PTMC/rGO conduit $(\mathrm{F}$ and $\mathrm{H})$ than in the PTMC conduit (E and $\mathrm{G})$. The same was found for the respective distal nerve stumps ( $\mathrm{J}$ and L compared to I and $\mathrm{K}$ ). As mentioned before, the regenerated tissue in the PTMC/rGO nerve guide was more form stable, which was probably caused by the higher amount of collagen present in this conduit as compared to the PTMC conduit. Both native sciatic nerves, the tissue present in the PTMC/rGO nerve guide as well as its distal nerve stump showed clusters of myelinated and non-myelinated axons surrounded by collagen, which were not seen in the PTMC conduit and its distal nerve stump. Hence, more nerve tissue had regenerated in the PTMC/rGO nerve guide than in the PTMC conduit, which resulted in a similar difference in the respective distal nerve stumps.

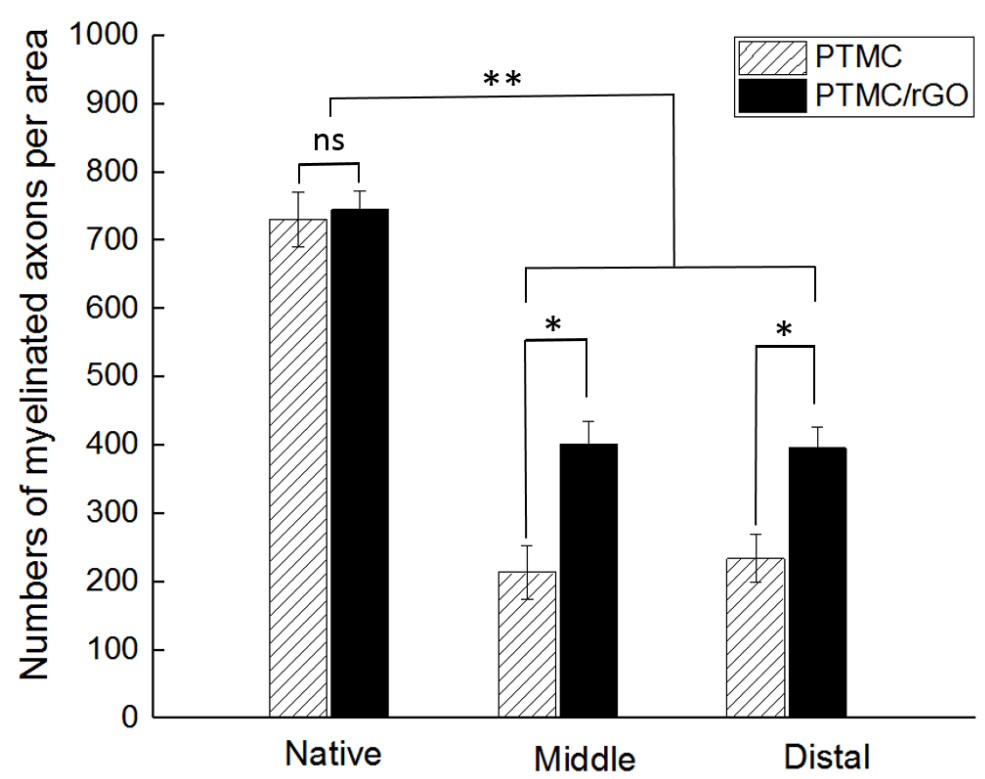

Figure 4. Numbers of myelinated axons in native sciatic nerve of the rabbit, in regenerated tissue in the middle of PTMC and PTMC/rGO nerve guide conduits, and in the respective distal nerve stumps. Of each cross-section, 4 areas of $200 \times 200 \mu m$ were evaluated. $* P<0.01, * * P<0.001$, ns: not significant. 
As action potentials are most efficiently transported along myelinated axons, the numbers of myelinated axons were counted in cross-sections of the native sciatic nerves, regenerated tissue in the middle of the conduits, and the distal nerve stumps (Figure 4). Whereas the numbers of myelinated axons were similar in the native sciatic nerves, they were significantly higher in the PTMC/rGO nerve guide than in the PTMC conduit. A similar difference of almost twice the amount was found for the respective distal nerve stumps. Compared to native sciatic nerve, the numbers of myelinated axons in the PTMC/rGO conduit and its distal nerve stump were significantly lower. Hence, during 6 weeks implantation in the rabbit, nerve tissue regenerated more extensively in the PTMC/rGO conduit, albeit not to the level of native sciatic nerve tissue.

Although the sciatic nerve defect regenerated to a larger extent using the PTMC/rGO conduit as compared to the PTMC nerve guide, there were no differences observed between the rabbits with respect to movement of the operated legs. After 6 weeks, both rabbits were able to move their leg at the side of the sciatic nerve defect, but not as good as the un-operated side. This can be explained in three ways. (1) Nerve regeneration in the PTMC/rGO conduit had not reached the threshold level for undisturbed leg movement. (2) Regenerated axons had not innervated the target muscles. (3) Regenerated axons had innervated the target muscles but were mislocated. These possibilities or combinations thereof will affect movement control and walking behavior of the animals [32]. For future studies, it is recommended to increase the amount of implantations as well as the implantation time, and to include walking track analysis and electrophysiological measurements.

\section{Conclusion}

Porous nerve guide conduits based on PTMC or electrically conductive PTMC/rGO composite were prepared by dip-coating, photo-crosslinking and particle leaching. Nerve regeneration in a rabbit sciatic nerve gap model was found to increase using the PTMC/rGO conduit as compared to the PTMC conduit. It is concluded that PTMC/rGO composite is an interesting material for the fabrication of conduits for peripheral nerve regeneration.

\section{Acknowledgements}

We thank the Chinese Scholarship Council for financial support and Huizhou Foryou Medical Devices Company for providing the TMC monomer. 


\section{References}

[1] A. Faroni, S. A. Mobasseri, P. J. Kingham, A.J. Reid, Peripheral nerve regeneration: experimental strategies and future perspectives, Adv. Drug Deliv. Rev., 82 (2015) 160-167.

[2] R. Li, Z. Liu, Y. Pan, L. Chen, Z. Zhang, L. Lu, Peripheral nerve injuries treatment: a systematic review, Cell Biochem. Biophys., 68 (2014) 449-454.

[3] A. Pabari, H. Lloyd-Hughes, A. M. Seifalian, A Mosahebi, Nerve conduits for peripheral nerve surgery, Plast. Reconstr. Surg., 133 (2014) 1420-1430.

[4] M. Gajendiran, J. Choi, S.J. Kim, K. Kim, H. Shin, H. J. Koo, K. Kim, Conductive biomaterials for tissue engineering applications, J. Ind. Eng. Chem., 51 (2017) 12-26.

[5] L. Ghasemi-Mobarakeh, M. P. Prabhakaran, M. Morshed, M. H. Nasr-Esfahani, H. Baharvand, S. Kiani, S. S. Al-Deyab, S. Ramakrishna, Application of conductive polymers, scaffolds and electrical stimulation for nerve tissue engineering, J. Tissue Eng. Regen. Med., 5 (2011) e17-e35.

[6] R. Balint, N. J. Cassidy, S. H. Cartmell, Conductive polymers: Towards a smart biomaterial for tissue engineering, Acta Biomater., 10 (2014) 2341-2353.

[7] G. Kaur, R. Adhikari, P. Cass, M. Bown, P. Gunatillake, Electrically conductive polymers and composites for biomedical applications, RSC Adv., 5 (2015) 37553-37567.

[8] P. Baei, S. Jalili-Firoozinezhad, S. Rajabi-Zeleti, M. Tafazzoli-Shadpour, H. Baharvand, N. Aghdami, Electrically conductive gold nanoparticle-chitosan thermosensitive hydrogels for cardiac tissue engineering, Mater. Sci. Eng. C Mater. Biol. Appl., 63 (2016) 131-141.

[9] A, Reznickova, Z. Novotna, O. Kvitek, Z. Kolska, V. Svorcik, Gold, silver and carbon nanoparticles grafted on activated polymers for biomedical applications, J. Nanosci. Nanotechnol., 15 (2015) 10053-10073.

[10] A. Bianco, K. Kostarelos, C. D. Partidos, M. Prato, Biomedical applications of functionalised carbon nanotubes, ChemComm., 5 (2005) 571-577.

[11] Y. Zhang, T. R. Nayak, H. Hong, W. Cai, Graphene: a versatile nanoplatform for biomedical applications, Nanoscale, 4 (2012) 3833-3842.

[12] D. Bitounis, H. Ali-Boucetta, B. H. Hong, D. H. Min, K. Kostarelos, Prospects and challenges of graphene in biomedical applications, Adv. Mater., 25 (2013) 2258-2268. 
[13] P. C. Sherrell, B. C. Thompson, J. K. Wassei, A. A. Gelmi, M. J. Higgins, R. B. Kaner, G. G. Wallace, Maintaining cytocompatibility of biopolymers through a graphene layer for electrical stimulation of nerve cells, Adv. Funct. Mater., 24 (2014) 769-776.

[14] Y. Qian, X. Zhao, Q Han, W. Chen, H. Li, W. Yuan, An integrated multi-layer 3Dfabrication of PDA/RGD coated graphene loaded PCL nanoscaffold for peripheral nerve restoration, Nat. Commun., 9 (2018) 323.

[15] S. Vijayavenkataraman, S. Thaharah, S. Zhang, WF. Lu, JY. Fuh, 3D-Printed PCL/rGO conductive scaffolds for peripheral nerve injury repair, Artif. Organs.,43 (2019) 515-523.

[16] W. Guo, S. Wang, X. Yu, J. Qiu, J. Li, W. Tang, Z. Li, X. Mou, H. Liu, Z. Wang, Construction of a 3D rGO-collagen hybrid scaffold for enhancement of the neural differentiation of mesenchymal stem cells, Nanoscale, 8 (2016) 1897-1904.

[17] J. Wang, Y. Cheng, L. Chen, T. Zhu, K. Ye, C. Jia, H. Wang, M. Zhu, C. Fan, X. Mo, In vitro and in vivo studies of electroactive reduced graphene oxide-modified nanofiber scaffolds for peripheral nerve regeneration, Acta Biomater., 84 (2019) 98-113.

[18] A. Muheremu, Q. Ao, Past, present, and future of nerve conduits in the treatment of peripheral nerve injury, Biomed. Res. Int., 2015 (2015) 237507.

[19] K. Fukushima, Poly(trimethylene carbonate)-based polymers engineered for biodegradable functional biomaterials, Biomater. Sci., 4 (2016) 9-24.

[20] J. J. Rongen, B. van Bochove, G. Hannink, D. W. Grijpma, P. Buma, Degradation behavior of, and tissue response to photo-crosslinked poly(trimethylene carbonate) networks, J. Biomed. Mater. Res. A, 104 (2016) 2823-2832.

[21] B. van Bochove, D. W. Grijpma, Photo-crosslinked synthetic biodegradable polymer networks for biomedical applications, J. Biomater. Sci. Polym. Ed., 30 (2019) 77-106.

[22] Z. Zhang, R. Kuijer, S. K. Bulstra, D. W. Grijpma, J. Feijen, The in vivo and in vitro degradation behavior of poly(trimethylene carbonate), Biomaterials, 27 (2006) 1741-1748.

[23] Y. Song, M. M. Kamphuis, Z. Zhang, L. T. Sterk, I. Vermes, A. A. Poot, J. Feijen, D. W. Grijpma, Flexible and elastic porous poly(trimethylene carbonate) structures for use in vascular tissue engineering, Acta Biomater., 6 (2010) 1269-1277. 
[24] S. G. Rotman, Z. Guo, D. W. Grijpma, A. A. Poot, Preparation and characterization of poly(trimethylene carbonate) and reduced graphene oxide composites for nerve regeneration, Polym. Adv. Technol., 28 (2017) 1233-1238.

[25] S. Park, J. An, I. Jung, R. D. Piner, S. J. An, X. Li, Velamakanni, R. S. Ruoff, Colloidal suspensions of highly reduced graphene oxide in a wide variety of organic solvents, Nano Lett., 9 (2009) 1593-1597.

[26] S. R. Aparicio, P. Marsden, A rapid methylene blue-basic fuchsin stain for semi-thin sections of peripheral nerve and other tissues, J. Microsc., 89 (1969) 139-141.

[27] W. F. den Dunnen, I. Stokroos, E. H. Blaauw, A. Holwerda, A. J. Pennings, P. H. Robinson, J. M. Schakenraad, Light-microscopic and electron-microscopic evaluation of short-term nerve regeneration using a biodegradable poly(DL-lactide-caprolacton) nerve guide, J. Biomed. Mater. Res., 31(1996) 105-115.

[28] W. F. den Dunnen, B. van der Lei, J. M. Schakenraad, E. H. Blaauw, I. Stokroos, A. J. Pennings, P. H. Robinson, Long-term evaluation of nerve regeneration in a biodegradable nerve guide, Microsurgery, 14 (1993) 508-515.

[29] M. F. Meek, P. H. Robinson, I. Stokroos, E. H. Blaauw, G. Kors, W. F. den Dunnen, Electronmicroscopical evaluation of short-term nerve regeneration through a thin-walled biodegradable poly(DLLA- $\varepsilon-C L)$ nerve guide filled with modified denatured muscle tissue, Biomaterials, 22 (2001) 1177-1185.

[30] S. H. Oh, J. H. Kim, K. S. Song, B. H. Jeon, J. H. Yoon, T. B. Seo, U. Namgung, I. W. Lee, J. H. Lee, Peripheral nerve regeneration within an asymmetrically porous PLGA/Pluronic F127 nerve guide conduit, Biomaterials, 29 (2008) 1601-1609.

[31] N. M. Bettahalli, H. Steg, M. Wessling, D. Stamatialis, Development of poly(l-lactic acid) hollow fiber membranes for artificial vasculature in tissue engineering scaffolds, J. Membr. Sci., 371 (2011) 117-126.

[32] M. F. Meek, W. F. den Dunnen, H. L. Bartels, A. J. Pennings, P. H. Robinson, J. M. Schakenraad, Peripheral nerve regeneration and functional nerve recovery after reconstruction with a thin-walled biodegradable poly(DL-lactide-e-caprolactone) nerve guide, Cells and Materials, 7 (1997) 53-62. 


\section{Chapter 5 - Synthesis and characterization of rGO-graft- poly(trimethylene carbonate) for nerve regeneration conduits}

Zhengchao Guo, Sandra Kofink, Honglin Chen, Jia Liang, Dirk W. Grijpma, André A. Poot

Department of Biomaterials Science and Technology, University of Twente, Enschede, The Netherlands.

Published

Z. Guo, S. Kofink, H. Chen, J. Liang, D.W. Grijpma, A.A. Poot, Synthesis and characterization of rGO-graft-poly(trimethylene carbonate) for nerve regeneration conduits, Biomed. Mater., 14 (2019) 034101. 


\begin{abstract}
Graphene-graft-polymer has been used to improve the compatibility between graphene and a polymer matrix, and to further enhance electrical, mechanical and biological properties of polymer/graphene composites. In this study, poly(trimethylene carbonate) (PTMC) was successfully grafted onto graphene surface via "grafting from" method. Reduced graphene oxide (rGO) initiator was synthesized by azido ethanol reaction with graphene oxide (GO) at high temperature. This resulted in thermal reduction of the GO and stable hydroxyl groups on the graphene surface. Subsequently, rGO initiator was used for the ring-opening polymerization of TMC monomer. rGO-graft-PTMC composites with PTMC molecular weights of 430,480, 2150 and $7030 \mathrm{~g} / \mathrm{mol}$ were successfully synthesized using different amounts of TMC. Single layer graphene nanosheets remained after graft polymerization by this method. rGO-graft-PTMC dispersions in chloroform were stable. The rGO-graft-PTMC composites with PTMC molecular weights of 430-7030 g/mol had electrical conductivities ranging from $0.2-0.016 \mathrm{~S} / \mathrm{cm}$. To investigate the biocompatibility of rGO-graft-PTMC, PTMCbased films containing rGO-graft-PTMC were prepared and used in cell culturing experiments. The composite films showed good biocompatibility with PC12 neuronal cells. It is concluded that rGO-graft-PTMC composite is a promising material for the preparation of nerve regeneration conduits.
\end{abstract}

\title{
1 Introduction
}

Peripheral nerve regeneration in the case of long-gap peripheral nerve damage remains a challenge [1][2]. At the moment there are three methods for the treatment of peripheral nerve injury. End-to-end suturing is the first method in which the two ends of nerve stumps are sewed together using a biodegradable suture. This method fails if the gap is too long. Then an alternative procedure, autologous nerve grafting can be applied. This is referred as the "gold standard" [3]. However, it comes with disadvantages as the number of donor nerves is limited and additional surgery is needed [4]. Hence, tubular nerve guide conduits have been developed to connect both nerve ends as a bridging device, providing a suitable environment for the regenerating nerve without scar tissue and compression from other tissues [5][6].

To prepare such a nerve guide conduit, a material with controlled biodegradability and biocompatibility is needed. Poly(ethylene glycol) (PEG) [7], poly( $\varepsilon$-caprolactone) (PCL) [8], 
poly(lactic acid) (PLA) [9] and poly(lactic-co-glycolic acid) (PLGA) [10] are well known polymers and have been used for the preparation of nerve guide conduits. Although promising results were obtained, these materials have drawbacks in terms of mechanical properties, degradation behavior and release of acidic degradation products, warranting the use of other polymers. Since nerve guide conduits are implanted by surgery it is required that the material can handle stress that occurs due to handling and suturing [11]. Additional properties are a low degree of swelling to reduce pressure on the nerve. Flexibility, permeability [12] and toughness [13] are required as well. Recently, poly(trimethylene carbonate) (PTMC) has attracted a lot of attention due to its excellent biocompatibility, surface degradation behavior and adjustable mechanical properties. Rubber-like PTMC can be synthesized by ring-opening polymerization of 1,3-trimethylene carbonate (TMC) and is already used for different biomedical applications [14]. For the fabrication of polymer structures such as nerve guide conduits, especially creepand tear-resistant PTMC networks are interesting [15].

Stimulation of neuronal cell growth and differentiation has been observed on electrically conductive interfaces. This initiated the use of electrically conductive materials in research on nerve regeneration [16]. Graphene in the form of graphene oxide (GO) and reduced graphene oxide (rGO) shows promising physical and chemical properties to promote neuronal cell growth [17][18]. Hence, graphene-based composites were used to fabricate nerve guide conduits which showed strong potential to restore nerve function [19-21]. In our previous study [22], rGO/PTMC composites showed electrical conductivity and facilitated the growth of neuronal cells. While preparing the graphene/polymer composites, however, irreversible aggregates of graphene layers formed due to van der Waals interactions [23]. This can be prevented by using functionalized graphene nanosheets in the form of graphene-graft-polymer [24]. Dispersion of graphene-graft-polymer in a polymer solution improves not only the processing ability but also the thermal stability and mechanical properties of the prepared structures [25-27]. "Graft to" and "Graft from" techniques have been used to synthesize graphene-graft-polymers. Generally, "Graft from" results in a higher graft density and is more likely to yield thin graphene sheets [28]. Graphene sheets with thermally stable hydroxyl groups, designated as rGO initiator, can be prepared by nitrene chemistry as reported by Gao [29]. In the present work, rGO-graft-PTMC composites with a varying PTMC content were synthesized by the ring-opening polymerization of TMC with $\mathrm{rGO}$ initiator. After physical and chemical characterization, including determination of electrical conductivity, the 
biocompatibility of PTMC-based composite films was investigated by culturing experiments with PC12 neuronal cells.

\section{Materials and methods}

\subsection{Materials}

Sulfuric acid $\left(\mathrm{H}_{2} \mathrm{SO}_{4}, 98.0 \%\right)$, phosphorus pentoxide (99\%), ammonium persulfate $(98.0 \%)$, potassium permanganate $(99.0 \%)$, hydrogen peroxide solution $\left(\mathrm{H}_{2} \mathrm{O}_{2}, 30 \%\right.$ in water), hydrochloric acid solution (HCL, $37 \%$ in water), 2-chloroethanol (99\%), sodium azide $(99.5 \%)$, stannous octoate $\left(\mathrm{Sn}(\mathrm{Oct})_{2}\right), 1$ - 4-(2-Hydroxyethoxy)-phenyl -2-hydroxy-2-methyl1-propane-1-one (Irgacure 2959) and magnesium sulfate $\left(\mathrm{MgSO}_{4}\right)$ were purchased from Sigma Aldrich, The Netherlands. $\mathrm{MgSO}_{4}$ was dried in an oven at $160^{\circ} \mathrm{C}$ before use, the other chemicals were used as delivered. Graphite flake (natural, -325 mesh, 99.8 \%) was acquired from Alfa Aesar, Germany. Diethyl ether, acetone, toluene and dichloromethane (DCM) were bought from VWR Chemicals, Germany. N-methyl-pyrrolidone (NMP) was purchased from Merck, Germany. TMC Monomer was kindly provided by Huizhou Foryou Medical Devices, China. PC12 cells (ATCC ${ }^{\circledR}$ CRL-1721 ${ }^{\mathrm{TM}}$ ) were ordered from ATCC, Germany. RPMI-1640 culture medium and horse serum were purchased from Sigma Aldrich. Fetal bovine serum and penicillin/streptomycin were obtained from Gibco. PrestoBlue ${ }^{\mathrm{TM}}$ Cell Viability Reagent was ordered from Thermo Fisher Scientific.

\subsection{Preparation of $\mathrm{rGO}$ initiator}

GO was synthesized by modified Hummers' method [30], as described in detail in our previous study [22]. A nitrogen chemistry reaction was performed to obtain thermally stable hydroxyl groups on the surface of the graphene layers. Homemade azido ethanol was used, synthesized according to [31]. Briefly, a $1000 \mathrm{~mL}$ three-neck-round-bottom flask was fitted with a condenser. Sodium azide $(97.5 \mathrm{~g} ; 1.5 \mathrm{~mol})$, deionized water $(390 \mathrm{~mL})$ and 2-chloroethanol ( $60.4 \mathrm{~g} ; 0.75 \mathrm{~mol})$ were added. The flask was heated to $75{ }^{\circ} \mathrm{C}$ in an oil bath and kept stirring for 96 hours. After cooling to room temperature, the product was extracted with diethyl ether $(5 \mathrm{x} 50 \mathrm{~mL})$. The extract was dried over anhydrous $\mathrm{MgSO}_{4}$ overnight. After filtering and evaporation of diethyl ether, azido ethanol was obtained as a colorless oil. 
Commonly, ring-opening polymerization of TMC is performed at high temperature in the bulk $[32,33]$. Thus, for the synthesis of rGO-graft-PTMC, thermally stable hydroxyl groups are needed to initiate the ring-opening polymerization of TMC. Therefore, GO was not used as initiator but rGO initiator was synthesized according to the method by Gao et al. [34] as shown in Scheme 1. In detail, $500 \mathrm{mg}$ GO and $200 \mathrm{~mL}$ NMP were put into a bottle. It was sonicated for 120 minutes ( $40 \mathrm{kHz}$, pre-experiments showed no formation of aggregates for this duration) and then placed in a $500 \mathrm{~mL}$ flask in an oil bath. After bubbling with nitrogen for 30 minutes, homemade azido ethanol $(10 \mathrm{~g})$ was added. The mixture was heated to $160{ }^{\circ} \mathrm{C}$ and kept at this temperature for 18 hours in argon atmosphere under constant stirring. During this step, GO was thermally reduced to $\mathrm{rGO}$ while reacting with azido ethanol to become rGO initiator. After cooling to room temperature, rGO initiator was separated by filtering the dispersion and washing with acetone. Finally, it was dried in a vacuum oven at room temperature until a constant weight was gained.

\subsection{Ring-opening polymerization of TMC with rGO initiator in toluene}

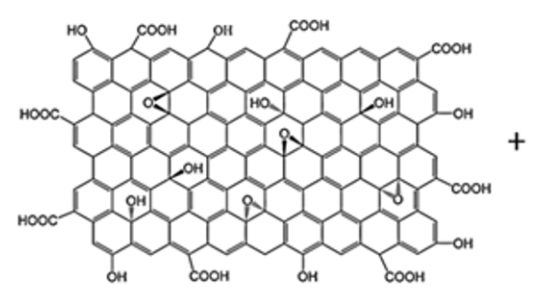

GO

rGO-graft-PTMC

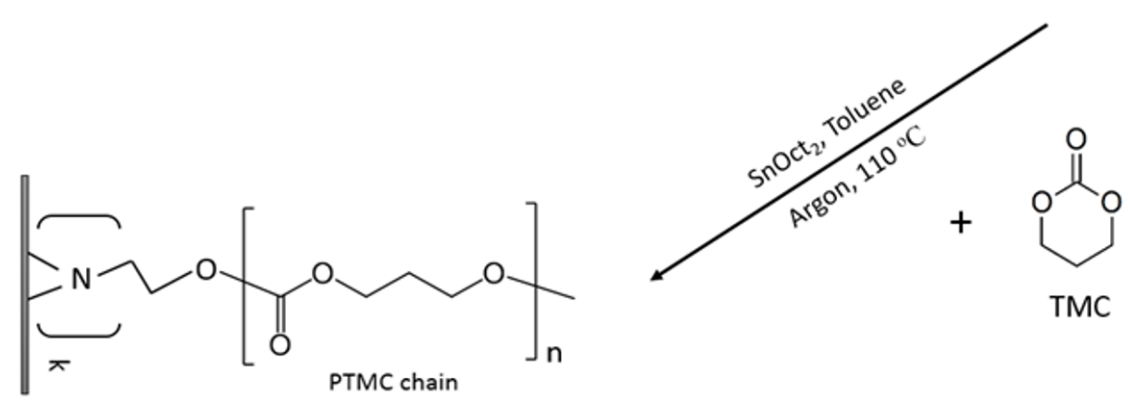

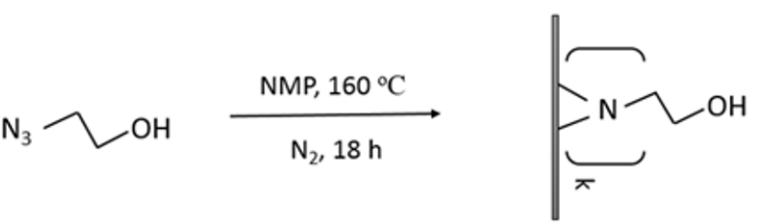

Azido ethanol

rGO initiator

Scheme 1. Synthesis of rGO initiator and ring-opening polymerization of TMC with rGO initiator.

Toluene was chosen to disperse rGO initiator and dissolve TMC for the ring-opening polymerization of TMC. Different amounts of TMC monomer (2.5, 5, 10 and $15 \mathrm{~g}), 0.05 \mathrm{~g}$ rGO initiator and $15 \mathrm{~mL}$ toluene were added into three-neck flasks and sonicated for 40 minutes. The dispersions were heated up to $80{ }^{\circ} \mathrm{C}$ in an oil bath and $\mathrm{Sn}(\mathrm{Oct})_{2}(0.02 \mathrm{~mol} / \mathrm{g}$ monomer $)$ 
was added as catalyst. The temperature was increased to $110{ }^{\circ} \mathrm{C}$ and left to react for 4 days under argon atmosphere with vigorous stirring, see Scheme 1. After reaction, the flasks were cooled to room temperature and toluene was poured out. To remove homo PTMC and residual TMC monomer, the mixture was washed with DCM and rGO-graft-PTMC was separated by centrifugation at $5000 \mathrm{rpm}$ for 10 minutes. This washing/centrifugation step was performed 5 times. Pure rGO-graft-PTMC was obtained by drying in the vacuum oven at room temperature until all DCM was removed.

\subsection{Characterization}

Fourier-transform infrared spectroscopy (FTIR, PerkinElmer Spectrum Two) was used to characterize the chemical structure of GO, rGO initiator and rGO-graft-PTMC.

Thermogravimetric analysis (TGA, PerkinElmer Pyris 1) of GO, rGO initiator and rGO-graftPTMC was performed with a temperature range from $50{ }^{\circ} \mathrm{C}$ to $550{ }^{\circ} \mathrm{C}$ and heating rate of $20{ }^{\circ} \mathrm{C} / \mathrm{min}$ under a nitrogen flow $(20 \mathrm{~mL} / \mathrm{min})$.

$\mathrm{X}$-ray diffractometry (XRD) was conducted on a PANalytical X'Pert Pro with $\mathrm{Cu}$-Ka radiation $(\lambda=0.15405 \mathrm{~nm})$ at $45 \mathrm{kV}$ and $40 \mathrm{~mA}$.

X-ray photoelectron spectroscopy (XPS, Physical Electronics Quantera SXM) was performed using an $\mathrm{Al} \mathrm{K} \alpha \mathrm{X}$-ray source $(1486.6 \mathrm{eV})$ and a vacuum pressure of $2.10^{-8}$ Torr. Multipack v.9.8 software was used for data analysis. For all these characterizations, dried powders of GO, rGO initiator and rGO-graft-PTMC were used.

\subsection{Morphology}

High resolution scanning electron microscopy (SEM, Zeiss MERLIN HR-SEM) and transmission electron microscopy (TEM, Philips CM300ST-FEG) were applied to observe the morphology of rGO initiator and rGO-graft-PTMC. To prepare the samples, diluted rGO initiator and rGO-graft-PTMC dispersions (around $0.05 \mathrm{mg} / \mathrm{mL}$ ) were prepared in chloroform and dropped on sample holders.

\subsection{Electrical Conductivity}

Electrical conductivity measurements were carried out by van der Pauw method $[35,36]$. To prepare the samples, rGO initiator and rGO-graft-PTMC were firstly dispersed in chloroform with 40 minutes sonication below $30^{\circ} \mathrm{C}$. Then the dispersions were filtered using $0.2 \mu \mathrm{m}$ pore 
size filter paper to get thin films, which were dried in the air and cut into $5 \mathrm{~mm}$ width and 5 $\mathrm{mm}$ length specimens with a graphene layer thickness of around $0.1 \mathrm{~mm}$.

\subsection{Biocompatibility}

2.7.1 Preparation of PTMC-based composite films as substrates for cell culturing

PTMC-based films containing either rGO-graft-PTMC or rGO initiator were prepared as follows. Photo-crosslinkable three-armed PTMC macromer with a molecular weight of 17,000 $\mathrm{g} / \mathrm{mol}$, functionalized with methacrylate end groups (PTMC-MA) was synthesized as previously reported [15]. Suspensions of rGO-graft-PTMC (sample code 2.5, see Table 1) and rGO initiator in chloroform were sonicated for 2 hours. Subsequently, PTMC-MA and photo initiator Irgacure 2959 (2.5 $\mathrm{wt} \%$ relative to PTMC-MA) were added to the dispersions. In both cases, the amount of rGO was $1 \mathrm{wt} \%$ relative to PTMC-MA. The mixtures were blended for 30 minutes and casted on glass plates to form thin films. The composite films were dried in the dark for 1 day at room temperature and then irradiated with $365 \mathrm{~nm}$ UV light for 30 minutes. Also plain PTMC films were prepared as a reference. All films were extracted twice with propylene carbonate:chloroform (7:3) solvent mixture, washed with ethanol and dried.

\subsubsection{PC12 cell culturing}

PC12 rat neuronal cells were cultured on the PTMC/rGO-graft-PTMC, PTMC/rGO initiator and plain PTMC films to evaluate their biocompatibility. The films were punched into $1 \mathrm{~cm}$ diameter circular samples and placed in 48 well suspension culture plates not treated for cell culture. Next, the films were stabilized with $12 \mathrm{~mm}$ diameter rubber rings to avoid floating and sterilized with $70 \%$ ethanol in a laminar flow hood. Empty wells were used as control. Samples for cell culturing were either non-coated or coated with $0.1 \mathrm{wt} \%$ gelatin solution (Sigma Aldrich) for $1 \mathrm{~h}$ before seeding the PC12 cells. Seeding density was 10,000 cells $/ \mathrm{cm}^{2}$, all samples were $n=4$. Complete culture medium consisted of 420 mL RPMI-1640 medium, 50 $\mathrm{mL}$ horse serum, $25 \mathrm{~mL}$ fetal bovine serum and $5 \mathrm{~mL}$ penicillin/streptomycin. Standard culture conditions of $37{ }^{\circ} \mathrm{C}$ and $5 \% \mathrm{CO}_{2}$ were applied. Culture media were completely changed every 2 days. At selected time points, PrestoBlue ${ }^{\mathrm{TM}}$ cell viability reagent was added to the culture medium directly after refreshing ( 0.1 vol PrestoBlue reagent to 0.9 vol culture medium). After incubation for $1 \mathrm{~h}$ at $37^{\circ} \mathrm{C}$, the fluorescence was read on a Tecan plate reader using excitation 
wavelength of $560 \mathrm{~nm}$ and emission wavelength of $590 \mathrm{~nm}$. After refreshing the medium, the PC12 cells were cultured until the next time point. Using separate samples, live/dead staining of PC12 cells was performed at day 7. Samples were rinsed with PBS and incubated with $2 \mu \mathrm{M}$ calcein-AM/4 $\mu \mathrm{M}$ ethidium homodimer-1 (Invitrogen) in PBS for $1 \mathrm{~h}$ at $37{ }^{\circ} \mathrm{C}$. The samples were rinsed with PBS and images were taken using an EVOS FL Cell Imaging System.

\section{Results and discussion}

\subsection{Synthesis and characterization of rGO-graft-PTMC}

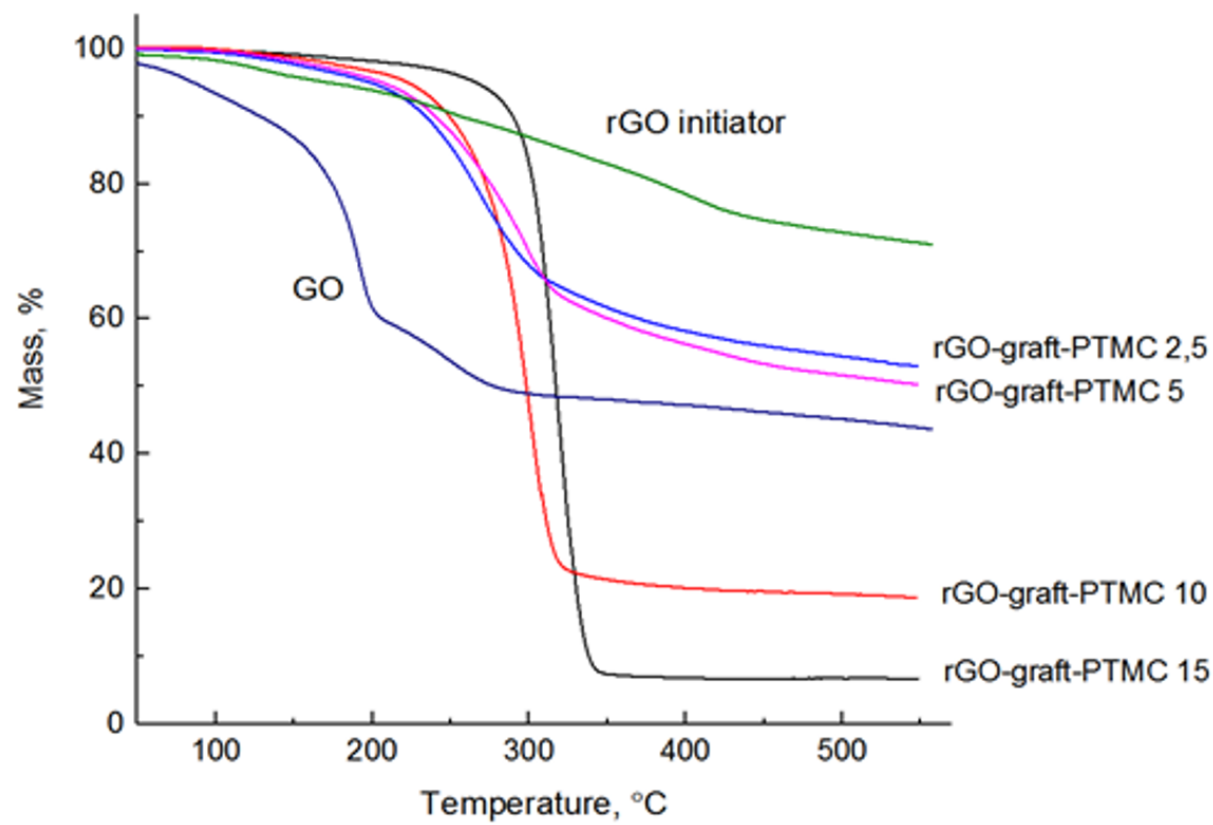

Figure 1. TGA curves of GO, rGO initiator and rGO-graft-PTMC. 2.5, 5, 10 and 15 refer to the amount of TMC (g) used for polymerization.

rGO-graft-PTMC was synthesized by ring-opening polymerization of TMC with rGO initiator in toluene. rGO initiator was used because of its thermal stability. Although there are large numbers of hydroxyl and carboxylic acid groups on the surface of GO, these groups are not thermally stable.

Thermogravimetric analysis of GO, rGO initiator and different types of rGO-graft-PTMC was performed, as shown in Figure 1. GO was not thermally stable as mass loss started below $110{ }^{\circ} \mathrm{C}$ due to loss of oxygen-containing groups like $-\mathrm{OH}$ and $-\mathrm{COOH}$. Similar results were 
reported by Stankovich et al. [36]. During synthesis of rGO initiator at $160{ }^{\circ} \mathrm{C}$, these unstable groups were removed, GO was thermally reduced and at the same time azido ethanol was bound to the surface yielding rGO initiator. As shown in Figure 1, there was no significant mass loss of rGO initiator below $110^{\circ} \mathrm{C}$, indicating that it was suitable to be used for the ring-opening polymerization of TMC at $110^{\circ} \mathrm{C}$. Grafted PTMC chains were degraded from $150{ }^{\circ} \mathrm{C}$ to $340^{\circ} \mathrm{C}$. The degradation temperature of rGO-graft-PTMC increased with increasing PTMC content. It was also found that the grafted PTMC chains increased the thermal stability of graphene layers. All rGO-graft-PTMC samples started to degrade at a higher temperature than rGO initiator. Similar results were found by Fang et al [23]. It was assumed that the weight loss of all rGOgraft-PTMC samples was completely due to decomposition of the PTMC chains. The initiator group content of rGO initiator as well as the PTMC content of the rGO-graft-PTMC composites could be estimated from the TGA measurements and were used to calculate the molecular weights of the grafted PTMC chains, as shown in Table 1.

Table 1. Synthesis and analysis of rGO-graft-PTMC

\begin{tabular}{|c|c|c|c|c|c|}
\hline Sample code & $\begin{array}{l}\text { rGO } \\
\text { initiator, } g\end{array}$ & $\begin{array}{l}\text { TMC } \\
\text { mass, g }\end{array}$ & $\begin{array}{l}\text { rGO-graft- } \\
\text { PTMC, g }\end{array}$ & $\begin{array}{l}\text { Residual content } \\
\text { at } 500{ }^{\circ} \mathrm{C}, \%\end{array}$ & $\begin{array}{l}\text { M }_{\text {PTMC }} \text { by } \\
\text { TGA, g/mol }\end{array}$ \\
\hline rGO-graft-PTMC 2.5 & 0.05 & 2.5 & 0.0729 & 54.5 & 430 \\
\hline rGO-graft-PTMC 5 & 0.05 & 5 & 0.0680 & 51.6 & 480 \\
\hline rGO-graft-PTMC 10 & 0.05 & 10 & 0.1407 & 19.3 & 2150 \\
\hline rGO-graft-PTMC 15 & 0.05 & 15 & 0.6396 & 6.8 & 7030 \\
\hline \multicolumn{6}{|c|}{$\begin{array}{l}\text { Initiator group content was } 13.3 \mathrm{wt} \% \text {, which was the weight loss of rGO initiator in the temperature } \\
\text { interval between } 240^{\circ} \mathrm{C} \text { and } 420^{\circ} \mathrm{C}[37] . \mathrm{M}_{\text {Inititor groups }} \text { was } \mathrm{N}-\mathrm{CH}_{2}-\mathrm{CH}_{2}-\mathrm{OH} \text { 's molar mass which is } 59 \\
\mathrm{~g} / \text { mol. Average molecular weight of grafted PTMC chains }\left(\mathrm{M}_{\mathrm{PTMC}}\right) \text { was calculated as follows. Mass } 500^{\circ} \mathrm{C} \\
\text { was the residual carbon content which was regarded as graphene mass without initiator groups. Mass start } \\
\text { was the weight of rGO-graft-PTMC used for the TGA measurement. }\end{array}$} \\
\hline
\end{tabular}

Four kinds of rGO-graft-PTMC with different PTMC molecular weights were synthesized as shown in Table 1. With more monomer loading, the PTMC content and the molecular weight 
of grafted PTMC chains increased. In addition, both parameters dramatically increased at a monomer feeding over $10 \mathrm{~g}$, which can be explained by a more efficient polymerization at a higher monomer concentration. The average molecular weights of PTMC chains grafted on the rGO surfaces were calculated using the TGA data, see Table 1 and Figure 1. The PTMC molecular weights of the 4 different rGO-graft-PTMC composites were 430, 480, 2150 and $7030 \mathrm{~g} / \mathrm{mol}$, respectively.
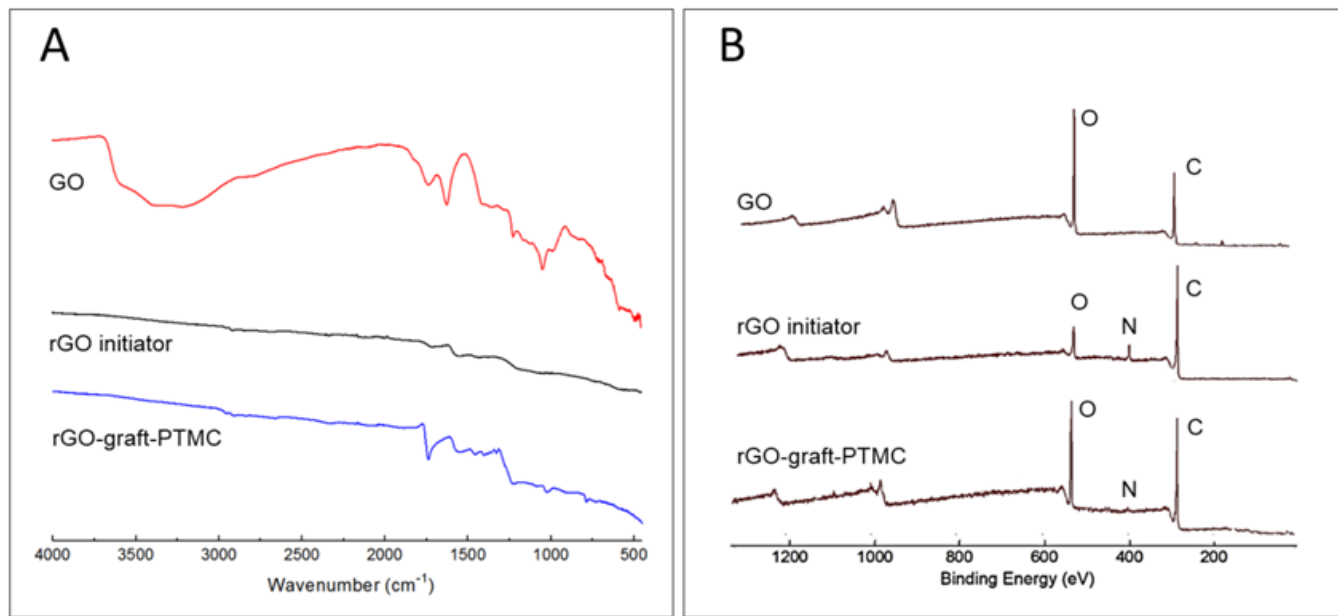

Figure 2. FTIR spectra (A) and XPS spectra (B) of GO, rGO initiator and rGO-graft-PTMC 2.5.

FTIR spectroscopy was used to chemically characterize the synthesized GO, rGO initiator and rGO-graft-PTMC, as shown in Figure 2A. Typical GO curves showed a broad band from 2900 to $3700 \mathrm{~cm}^{-1}$, which was the stretching peak of different hydroxyl groups. The peaks at 1716 , 1635,1152 and $1030 \mathrm{~cm}^{-1}$ were due to stretching of $\mathrm{C}=\mathrm{O}, \mathrm{C}=\mathrm{C}, \mathrm{C}-\mathrm{OH}$ and $\mathrm{C}-\mathrm{O}$ bonds, respectively. rGO initiator curves showed that unstable hydroxyl groups $\left(2900-3700 \mathrm{~cm}^{-1}\right)$ of GO were removed during azido ethanol reaction at high temperature. The peak around 1586 $\mathrm{cm}^{-1}$ belonged to $\mathrm{C}=\mathrm{C}$ and the one at $1164 \mathrm{~cm}^{-1}$ referred to remaining $\mathrm{C}-\mathrm{OH}$ groups. Similar peaks were reported for thermally reduced graphene [38]. The FTIR spectrum of rGO-graftPTMC showed C-O $\left(1230 \mathrm{~cm}^{-1}\right), \mathrm{C}=\mathrm{O}\left(1700 \mathrm{~cm}^{-1}\right)$ and $\mathrm{C}-\mathrm{H}\left(2900 \mathrm{~cm}^{-1}\right)$ peaks referring to PTMC chains grafted on the surface of rGO initiator.

XPS spectra of GO, rGO initiator and rGO-graft-PTMC are shown in Figure 2B. In contrast to $\mathrm{GO}, \mathrm{rGO}$ initiator showed a strong nitrogen signal, indicating that azido ethanol was coupled to the graphene surface. As compared to rGO initiator, rGO-graft-PTMC showed a stronger 
oxygen signal due to the presence of PTMC. The $\mathrm{C}, \mathrm{N}$ and $\mathrm{O}$ atomic percentages of GO, rGO initiator and rGO-graft-PTMC are given in Table 2. As compared to GO, a significant nitrogen content was present in the case of rGO initiator. Thermal reduction of GO during synthesis of rGO initiator resulted in a strong decrease of oxygen content from 38.78 to $11.89 \%$, which increased to $20.80 \%$ upon graft polymerization of PTMC. The atomic percentages shown in Table 2 for rGO-graft-PTMC were determined after 5 times washing of the composite with DCM, in order to remove any adsorbed PTMC from the graphene surface. Additional 5 times washing of the rGO-graft-PTMC with DCM did not change the $\mathrm{C}, \mathrm{N}$ and $\mathrm{O}$ atomic percentages (data not shown). Taken together, this demonstrates that PTMC was successfully grafted onto the rGO surface.

Table 2. Atomic content of GO, $r G O$ initiator and rGO-graft-PTMC as determined by XPS $(n=4)$.

\begin{tabular}{lccc}
\hline Atomic content, $\%$ & $\mathbf{C}$ & $\mathbf{N}$ & $\mathbf{O}$ \\
\hline GO & $59.33 \pm 0.16$ & $0.18 \pm 0.11$ & $38.78 \pm 0.25$ \\
\hline rGO initiator & $82.28 \pm 0.46$ & $5.51 \pm 0.28$ & $11.89 \pm 0.12$ \\
\hline rGO-graft-PTMC 2.5 & $75.42 \pm 0.50$ & $1.29 \pm 0.21$ & $20.80 \pm 0.45$ \\
\hline
\end{tabular}

XRD was used to verify exfoliation of graphene nanosheets in GO, rGO initiator and rGOgraft-PTMC. As shown in Figure 3, the GO pattern showed a strong peak around $10.1^{\circ}$, indicating the presence of oxygen-containing structures formed during oxidation. This strong peak disappeared in the patterns of rGO initiator and rGO-graft-PTMC, which is in agreement with thermal reduction during the synthesis of rGO initiator. The rGO initiator pattern showed a broad peak around $24.9^{\circ}$, similar to the pattern for thermally reduced graphene oxide reported by Mishra et al. [39]. The absence of a strong peak in the rGO initiator pattern indicates that the GO nanosheets remained exfoliated during the nitrene chemistry reaction [23]. The rGOgraft-PTMC pattern showed almost no visible peaks, suggesting stabilization of exfoliated rGO nanosheets during graft polymerization. Similar results were reported by Fang et al. [40]. 


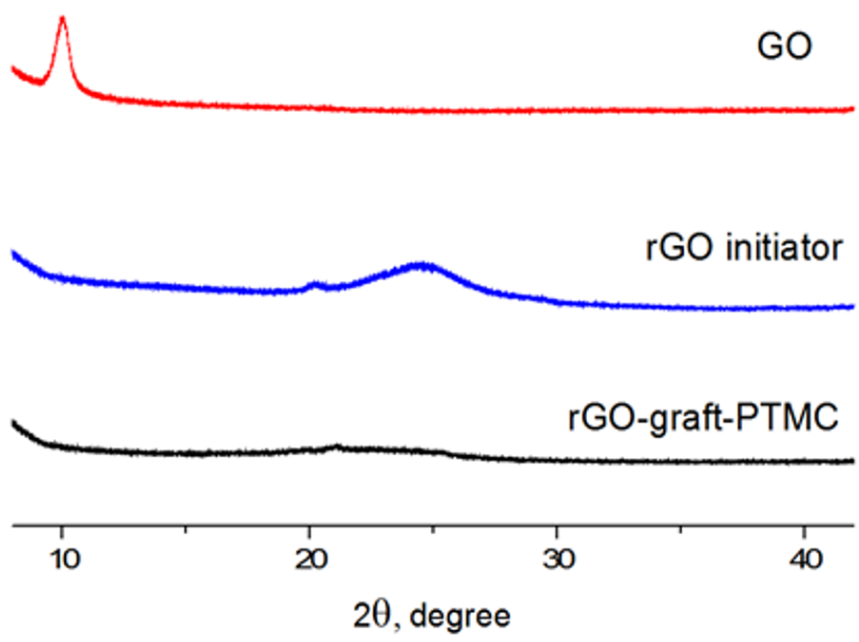

Figure 3. XRD curves of $G O$, rGO initiator and rGO-graft-PTMC 2.5.

\subsection{Dispersion behavior}

The dispersion behavior of GO, rGO initiator and rGO-graft-PTMC was investigated in water and chloroform, see Figure 4. The same concentration $(0.5 \mathrm{mg} / \mathrm{mL})$ was used for all samples. GO could be dispersed very well in water due to the large amount of hydroxyl groups resulting in its hydrophilic character. rGO initiator was not able to form a stable dispersion in water due to its relatively high hydrophobicity [41]. rGO-graft-PTMC could not be dispersed at all in water due to the hydrophobic PTMC chains on the graphene surface. In contrast to GO, rGO initiator and rGO-graft-PTMC could be stably dispersed in chloroform, which is a nonpolar solvent. To prepare a polymer/graphene composite, rGO initiator or rGO can easily form aggregates when it is mixed with polymer at a relatively high concentration. Grafted polymer chains on the surface of graphene increase the compatibility of the graphene with the polymer [42]. In the present study, grafting of PTMC chains on the surface of rGO nanosheets was applied to prepare homogenous PTMC/rGO-graft-PTMC composites using chloroform as a solvent. 


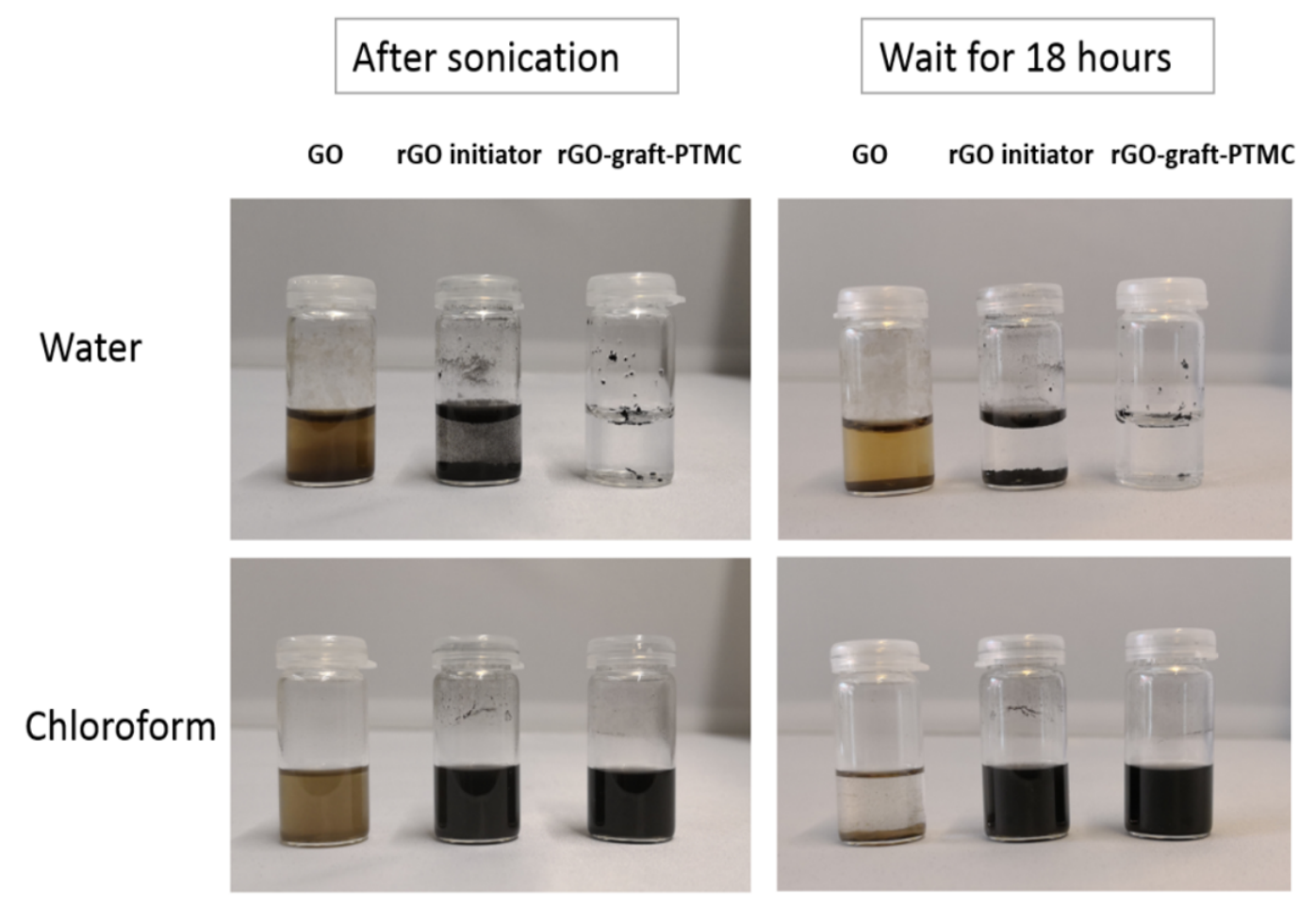

Figure 4. Dispersion behavior of GO, rGO initiator and rGO-graft-PTMC 2.5 in water and chloroform

\subsection{Morphology}

The morphology of rGO initiator and rGO-graft-PTMC was investigated using both SEM and TEM. As shown in Figure 5A, the surface of rGO initiator was relatively smooth and clean. The surface of rGO-graft-PTMC was rough due to the presence of a lot of PTMC particles (Figure 5B). This again proved that PTMC chains were successfully grafted on the surface of the rGO nanosheets. As shown in Figure 5C and D, single nanosheets of rGO initiator and rGO-graft-PTMC were present. Thus, single layer rGO nanosheets remained during the synthesis of graphene-graft-polymer. 

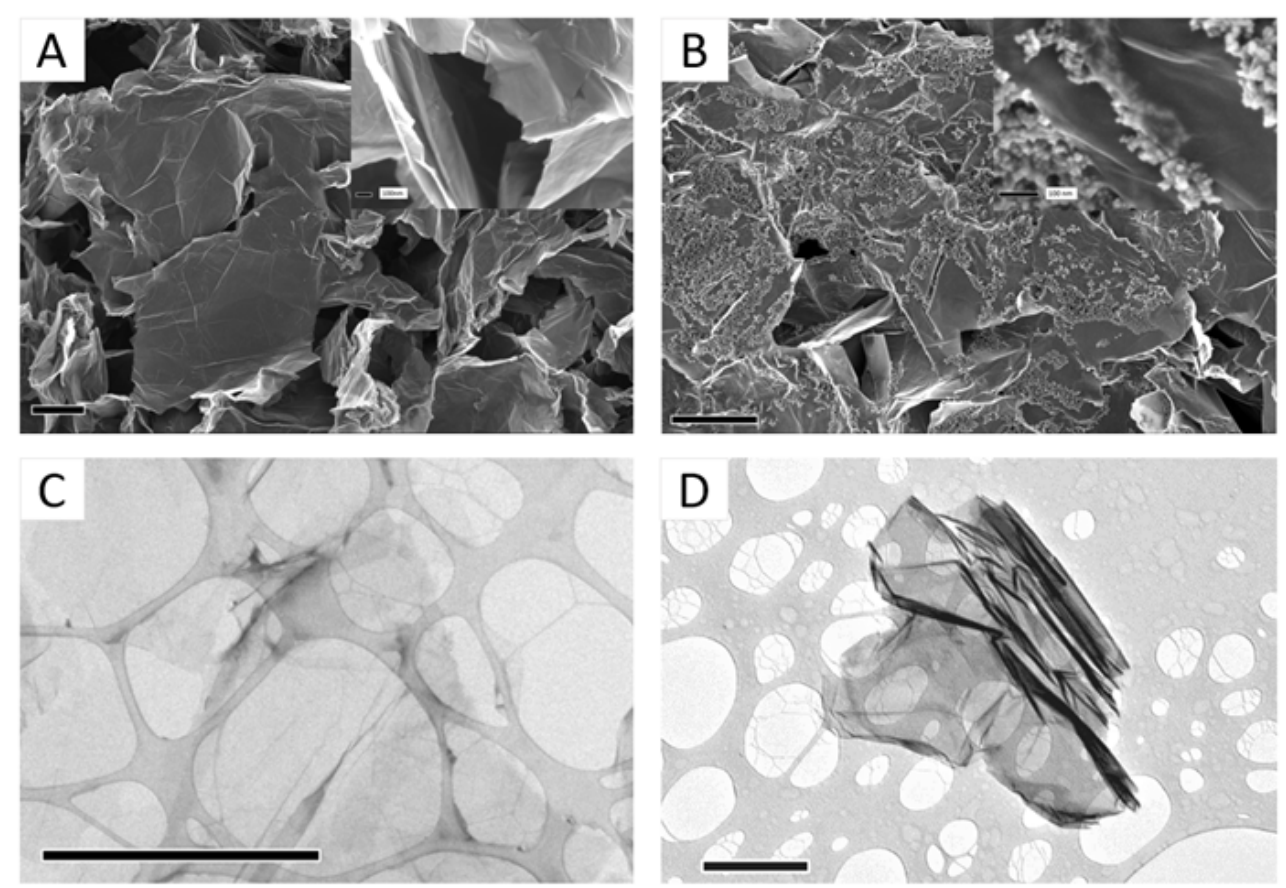

Figure 5. SEM images of $r G O$ initiator (A) and $r G O$-graft-PTMC 2.5 (B), TEM images of $r G O$ initiator (C) and rGO-graft-PTMC $2.5(D)$, scale bar is $2 \mu \mathrm{m}$.

\subsection{Conductivity}

Electrical conductivity as the most important property of graphene derivatives was characterized as well, see Table 3. The electrical conductivity of graphene-based materials is related to their carbon content. In our case, a higher residual content at $500{ }^{\circ} \mathrm{C}$ indicates a higher amount of rGO in the rGO-graft-PTMC composites. As rGO initiator was thermally reduced, it showed a relatively high conductivity of $1.59 \mathrm{~S} / \mathrm{cm}$, close to the value reported for highly reduced graphene oxide [43]. Regarding rGO-graft-PTMC, the conductivity values decreased from 0.2 to $0.016 \mathrm{~S} / \mathrm{cm}$ with decreasing $\mathrm{rGO}$ content. It was noticed that the rGO-graft-PTMC 15 sample with $6.8 \mathrm{wt} \%$ graphene showed a similar conductivity $\left(\sim 10^{-2} \mathrm{~S} / \mathrm{cm}\right)$ as reported for $\mathrm{PCL} / \mathrm{rGO}$ composites with $10 \mathrm{wt} \%$ graphene [44]. This rGO-graft-PTMC with high PTMC content could be directly used or further processed as a PTMC/rGO-graft-PTMC composite for the preparation of nerve guide conduits. 
Table 3. Conductivity of $r G O$ initiator and $r G O$-graft-PTMC

\begin{tabular}{lccc}
\hline Sample & $\begin{array}{l}\text { Residual content } \\
\mathbf{5 0 0}{ }^{\circ} \mathbf{C}, \boldsymbol{\%}\end{array}$ & $\begin{array}{l}\text { Conductivity, } \\
\mathbf{S} / \mathbf{c m}\end{array}$ & Reference \\
\hline $\begin{array}{l}\text { rGO film (hydrazine- } \\
\text { reduced) }\end{array}$ & - & 2.53 & {$[43]$} \\
\hline $\begin{array}{l}\text { rGO initiator (thermally } \\
\text { reduced) }\end{array}$ & 78.2 & 1.59 & This work \\
\hline rGO-graft-PTMC 2.5 & 54.5 & 0.20 & This work \\
\hline rGO-graft-PTMC 5 & 51.6 & 0.158 & This work \\
\hline rGO-graft-PTMC 10 & 19.3 & 0.044 & This work \\
\hline rGO-graft-PTMC 15 & 6.8 & 0.016 & This work \\
\hline
\end{tabular}

\subsection{Biocompatibility}

A

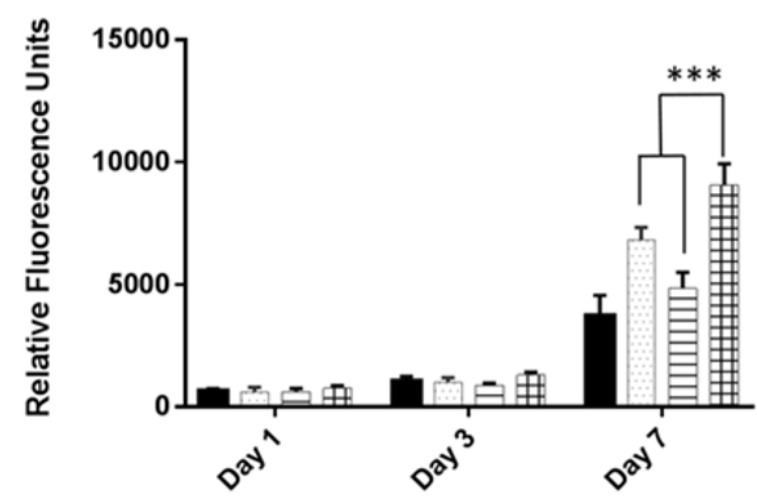

Non-coated culture plate

5 PTMC

$\boxminus$ PTMC/rGO initiator

巴 PTMC/rGO-graft-PTMC

B

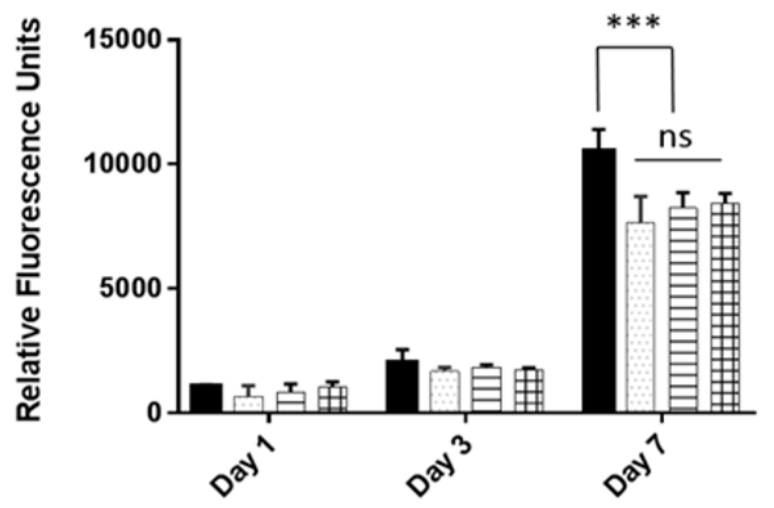

Coated culture plate

$\square$ PTMC

$\Xi \mathrm{PTMC} / \mathrm{rGO}$ initiator

册 PTMC/rGO-graft-PTMC

Figure 6. PC12 cell viability on non-coated (A) and gelatin-coated (B) culture plates, PTMC, PTMC/rGO initiator and PTMC/rGO-graft-PTMC composite films after 1, 3 and 7 days of culturing. The data were analyzed by two-way ANOVA. ***p<0.001, ns: no significant difference. As graphenegraft-polymer, rGO-graft-PTMC 2.5 was used. 
To investigate the biocompatibility of rGO-graft-PTMC, PC12 cells were cultured on noncoated and gelatin-coated PTMC/rGO-graft-PTMC composite films. Plain PTMC films and $\mathrm{PTMC} / \mathrm{rGO}$ initiator composite films were tested as well, and wells of non-tissue culturetreated culture plates were used as control. As shown in Figure 6A for non-coated surfaces, there were no differences in cell viability on all surfaces up to 3 days of culturing. On day 7 , however, cell viabilities on the PTMC-based films were higher than on the culture plate. Moreover, cells cultured on PTMC/rGO-graft-PTMC films showed a significantly higher cell viability as compared to cells cultured on PTMC and PTMC/rGO initiator films. Also for gelatin-coated surfaces (Figure 6B), there were only differences in cell viability on day 7. In this case, cell viabilities on the PTMC-based films were similar, and significantly lower than on the culture plate.

These results were confirmed by live/dead imaging of the cells on day 7. As shown in Figure 7 , a very low amount of dead cells was observed on all surfaces. In the case of non-coated surfaces, relatively low amounts of cells were observed on the culture plate and on PTMC/rGO initiator film, whereas cell numbers on PTMC and especially PTMC/rGO-graft-PTMC films were higher. All gelatin-coated surfaces showed high cell numbers, with the highest amount of cells on the culture plate. These results indicate that rGO-graft-PTMC composite is biocompatible and suitable for future application in peripheral nerve regeneration.

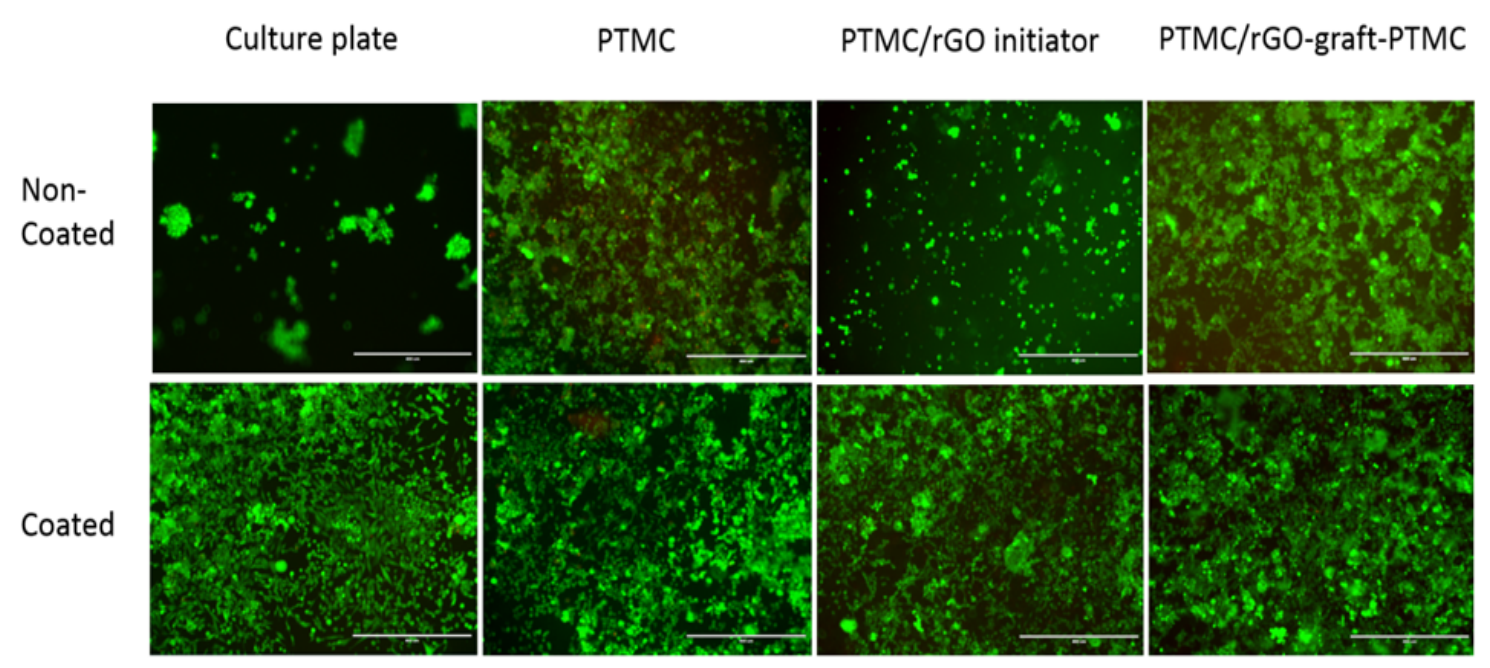

Figure 7. Live/dead staining of PC12 cells on non-coated (top) and gelatin-coated (bottom) culture plates, PTMC, PTMC/rGO initiator and PTMC/rGO-graft-PTMC composite films after 7 days of culturing. Green: live cells, red: dead cells. Scale bar $400 \mu m$. 


\section{Conclusion}

rGO-graft-PTMC was successfully synthesized by ring-opening polymerization of TMC with rGO initiator. During synthesis of rGO initiator by reaction of GO with azido ethanol at high temperature, the GO was thermally reduced. Different PTMC chain lengths were obtained by using different amounts of TMC monomer in the ring-opening polymerization. During the various steps of this method, single layer graphene nanosheets remained. rGO-graft-PTMC was electrically conductive and biocompatible in culturing experiments with PC12 neuronal cells. It is concluded that rGO-graft-PTMC composite is a promising material for the preparation of nerve regeneration conduits.

\section{Acknowledgments}

The authors would like to thank Huizhou Foryou Medical Company for kindly providing TMC monomer and the Chinese Scholarship Council for financial support. The authors also appreciate Jun Wang, Yang Wang and Shaochen Zhu for their help with conductivity testing and XRD measurements.

\section{References}

[1] X. Wang, K-F. So, X. Xu, Advances and challenges for neural regeneration research, Neural Regeneration, 1 (2015) 3-17.

[2] T.A. Ferguson, G.M. Smith, Conditions affecting accuracy of peripheral nerve reinnervation and functional recovery, Neural Regeneration, 7 (2015) 117-128.

[3] R. Deumens, A. Bozkurt, M.F. Meek, M.A.E. Marcus, E.A..J. Joosten, J. Weis, GA. Brook, Repairing injured peripheral nerves: Bridging the gap, Prog Neurobiol, 92 (2010) 245-276.

[4] V. Chiono, C. Tonda-Turo, Trends in the design of nerve guidance channels in peripheral nerve tissue engineering, Prog Neurobiol., 131 (2015) 87-104

[5] M. Georgiou, S.C.J. Bunting, H.A. Davies, A.J. Loughlin, J.P. Golding, J.B. Phillips, Engineered neural tissue for peripheral nerve repair, Biomaterials, 34 (2013) 7335-7343. 
[6] X.S. Gu, F. Ding, D.F. Williams, Neural tissue engineering options for peripheral nerve regeneration, Biomaterials, 35 (2014) 6143-6156.

[7] C.J. Pateman, AJ. Harding, Glen A, C.S. Taylor, C.R. Christmas, P.P. Robinson, Rimmer S, F.M. Boissonade, F. Claeyssens, J.W. Haycock, Nerve guides manufactured from photocurable polymers to aid peripheral nerve repair, Biomaterials, 49 (2015) 77-89.

[8] A.J. Reid, A.C. de Luca, A. Faroni, S. Downes, M.Z. Sun, G. Terenghi, P.J. Kingham, Long term peripheral nerve regeneration using a novel PCL nerve conduit, Neurosci. Lett., 544 (2013) 125-130.

[9] F. Xie, F.Q. Li, B. Gu, K. Liu, G.X. Shen, In vitro and in vivo evaluation of a biodegradable chitosan-PLA composite peripheral nerve guide conduit material, Microsurg., 28 (2008) 471 479.

[10] S.H. Oh, J.H. Lee, Fabrication and characterization of hydrophilized porous PLGA nerve guide conduits by a modified immersion precipitation method, J. Biomed. Mater. Res. A, 80a (2007) 530-538.

[11] M. Farokhi, Mottaghitalab F, M.A. Shokrgozar, D.L. Kaplan, H.W. Kim, S.C. Kundu, Prospects of peripheral nerve tissue engineering using nerve guide conduits based on silk fibroin protein and other biopolymers, Int. Mater. Rev., 62 (2017) 367-391.

[12] A. Adamus, R.A. Wach, A.K. Olejnik, J. Dzierzawska, J.M. Rosiak, Degradation of nerve guidance channels based on a poly(L-lactic acid) poly(trimethylene carbonate) biomaterial, Polym. Degrad. Stabil, 97 (2012) 532-540.

[13] A.R. Nectow, K.G. Marra, D.L. Kaplan, Biomaterials for the development of peripheral nerve guidance conduits, Tissue Eng. Part B-Re., 18 (2012) 40-50.

[14] K. Fukushima, Poly(trimethylene carbonate)-based polymers engineered for biodegradable functional biomaterials, Biomater. Sci., 4 (2016) 9-24.

[15] S. Schuller-Ravoo, J. Feijen, D.W. Grijpma, Flexible, elastic and tear-resistant networks prepared by photo-crosslinking poly(trimethylene carbonate) macromers, Acta Biomater., 8 (2012) 3576-3585.

[16] T. Nezakati, B.G. Cousins, A.M. Seifalian, Toxicology of chemically modified graphenebased materials for medical application, Arch. Toxicol., 88 (2014) 1987-2012. 
[17] M. Tang, Q. Song, N. Li, Z. Jiang, R. Huang, G. Cheng, Enhancement of electrical signaling in neural networks on graphene films, Biomaterials, 34 (2013) 6402-411.

[18] Y. Zhu, S. Murali, W. Cai, X. Li, J.W. Suk, J.R. Potts, RS. Ruoff, Graphene and graphene oxide: synthesis, properties, and applications, Adv. Mater., 22 (2010) 3906-3924.

[19] Y. Kong, Y. Zhao, B. Ji, B. Shi, S. Wei, G. Chen, L. Zhang, G. Li, Y. Yang, Preparation and characterization of polyacrylamide/silk fibroin/graphene oxide composite hydrogel for peripheral nerve regeneration, J. Biomater. Tissue Eng., 6 (2016) 682-689.

[20] Y. Qian, X. Zhao, Q. Han, W. Chen, H. Li, W. Yuan, An integrated multi-layer 3Dfabrication of PDA/RGD coated graphene loaded PCL nanoscaffold for peripheral nerve restoration, Nat. Commun., 9 (2018) 323.

[21] Z. Feng, T. Wang, B. Zhao, J. Li, L. Jin, Soft Graphene Nanofibers Designed for the Acceleration of Nerve Growth and Development, Adv. Mater., 27 (2015) 6462-6468.

[22] S.G. Rotman, Z. Guo, D.W. Grijpma, A.A. Poot, Preparation and characterization of poly(trimethylene carbonate) and reduced graphene oxide composites for nerve regeneration, Polym. Adv. Technol., 28 (2017) 1233-1238.

[23] M. Fang, K. Wang, H. Lu, Y. Yang, S. Nutt, Covalent polymer functionalization of graphene nanosheets and mechanical properties of composites, J Mater. Chem, 19 (2009) 70987105 .

[24] Y. Min, M. Akbulut, K. Kristiansen, Y. Golan, J. Israelachvili, The role of interparticle and external forces in nanoparticle assembly, Nat. Mater., 7 (2008) 527-538.

[25] M. Wang, N. Hu, L. Zhou, C. Yan, Enhanced interfacial thermal transport across graphene-polymer interfaces by grafting polymer chains, Carbon, 85 (2015) 414-421

[26] H.J. Salavagione, G. Martinez, G. Ellis, Recent advances in the covalent modification of graphene with polymers, Macromol. Rapid. Comm., 32 (2011) 1771-1789

[27] Z. Xu, C. Gao, In situ Polymerization approach to graphene-reinforced nylon-6 composites, Macromolecules, 43 (2010) 6716-6723.

[28] R.K. Layek, A.K. Nandi, A review on synthesis and properties of polymer functionalized graphene, Polymer, 54 (2013) 5087-50103. 
[29] H. He, C. Gao, General approach to individually dispersed, highly soluble, and conductive graphene nanosheets functionalized by nitrene chemistry, Chem. Mater., 22 (2010) 5054-5064.

[30] S. Park, R.S. Ruoff, Chemical methods for the production of graphenes, Nat. Nanotechnol., 4 (2009) 217-224.

[31] B.S. Sumerlin, N.V. Tsarevsky, G. Louche, R.Y. Lee, K. Matyjaszewski, Highly efficient "click" functionalization of poly(3-azidopropyl methacrylate) prepared by ATRP, Macromolecules, 38 (2005) 7540-7545.

[32] K. Zhu, R.W. Hendren, K. Jensen, C.G. Pitt, Synthesis, properties, and biodegradation of poly(1,3-trimethylene carbonate), Macromolecules, 24 (1991) 1736-1740.

[33] Q. Hou, DW. Grijpma, J. Feijen, Creep-resistant elastomeric networks prepared by photocrosslinking fumaric acid monoethyl ester-functionalized poly(trimethylene carbonate) oligomers, Acta Biomater., 5 (2009) 1543-1551.

[34] C. Gao, H. He, L. Zhou, X. Zheng, Y. Zhang, Scalable functional group engineering of carbon nanotubes by improved one-step nitrene chemistry, Chem. Mater., 21 (2009) 360-370.

[35] L.J. van der Pauw, A method of measuring specific resistivity and Hall effect of discs of arbitrary shape, Philips Research reports, 13 (1958) 1-9.

[36] S. Stankovich, D.A. Dikin, R.D. Piner, KA. Kohlhaas, A. Kleinhammes, Y. Jia, Y. Wu, S.T. Nguyen, R.S. Ruoff, Synthesis of graphene-based nanosheets via chemical reduction of exfoliated graphite oxide, Carbon, 45 (2007) 1558-1565.

[37] H. Zeng, C. Gao, D. Yan, Poly(epsilon-caprolactone)-functionalized carbon nanotubes and their biodegradation properties, Adv. Funct. Mater., 16 (2006) 812-818.

[38] Y. Gong, D. Li, Q. Fu, C. Pan, Influence of graphene microstructures on electrochemical performance for supercapacitors, Prog. Nat. Sci. Mater. Int., 25 (2015) 379-385.

[39] S.K. Mishra, S.N. Tripathi, V. Choudhary, B.D. Gupta, SPR based fibre optic ammonia gas sensor utilizing nanocomposite film of PMMA/reduced graphene oxide prepared by in situ polymerization, Sens. Actuators B Chem., 199 (2014) 190-200.

[40] M. Fang, K. Wang, H. Lu, Y. Yang, S. Nutt, Single-layer graphene nanosheets with controlled grafting of polymer chains, J. Mater. Chem, 20 (2010) 1982-1992. 
[41] M. Song, Y. Yun, N.R. Kim, H. Jin, Dispersion stability of chemically reduced graphene oxide nanoribbons in organic solvents, Rsc. Adv., 6 (2016) 19389-19393.

[42] L. Tang, Y. Wan, D. Yan, Y. Pei, L. Zhao, Y. Li, L. Wu, J. Jiang, G. Lai, The effect of graphene dispersion on the mechanical properties of graphene/epoxy composites, Carbon, 60 (2013) 16-27.

[43] S. Park, J.W. Suk, J.H. An, J. Oh, S. Lee, W. Lee, J.R. Potts, J.H. Byun, R.S. Ruoff, The effect of concentration of graphene nanoplatelets on mechanical and electrical properties of reduced graphene oxide papers, Carbon, 50 (2012) 4573-4578.

[44] S. Sayyar, E. Murray, B.C. Thompson, S. Gambhir, D.L. Officer, G.G. Wallace, Covalently linked biocompatible graphene/polycaprolactone composites for tissue engineering, Carbon, 52 (2013) 296-304. 


\section{Chapter 6 - Fabrication of poly(trimethylene carbonate)/reduced graphene oxide-graft-poly(trimethylene carbonate) composite scaffolds for nerve regeneration}

Zhengchao Guo, André A. Poot, Dirk W. Grijpma, Honglin Chen

Department of Biomaterials Science and Technology, University of Twente, Enschede, The Netherlands.

Published

Z. Guo, J. Liang, M.J. Ankone, A.A. Poot, D.W. Grijpma, H. Chen, Fabrication of poly (trimethylene carbonate)/reduced graphene oxide-graft-poly(trimethylene carbonate) composite scaffolds for nerve regeneration, Biomed. Mater., 14 (2019) 024104. 


\begin{abstract}
One of the key challenges for neural tissue engineering is to exploit functional materials to guide and support nerve regeneration. Currently, reduced graphene oxide (rGO) which is wellknown for its unique electrical and mechanical properties has been incorporated into biocompatible polymers to manufacture functional scaffolds for nerve tissue engineering. However, rGO has poor dispersity in polymer matrix, which limits its further application. Here, we replaced rGO with rGO-graft-PTMC. The rGO-graft-PTMC was firstly prepared by grafting trimethylene carbonate (TMC) oligomers onto rGO. And then, PTMC/rGO-graftPTMC composite fibrous mats were fabricated by electrospinning of a dispersion of PTMC and rGO-graft-PTMC. The loading of rGO-graft-PTMC could reach up to $6 \mathrm{wt} \%$ relative to PTMC. Scanning electron microscopy (SEM) images showed that the morphologies and average diameters of PTMC/rGO-graft-PTMC composite fibrous mats were affected by the content of rGO-graft-PTMC. Additionally, the incorporation of rGO-graft-PTMC resulted in enhanced thermal stability and hydrophobicity of PTMC fibers. Biological results demonstrated that PC12 cells showed higher cell viability in PTMC/rGO-graft-PTMC fibers of $1.2 \%$ and $6.0 \mathrm{wt} \% \mathrm{rGO}$-graft-PTMC compared to pure PTMC fibers. These results suggested that PTMC/rGO-graft-PTMC composite fibrous mats hold great potential as conduits for neural tissue engineering.
\end{abstract}

\title{
1 Introduction
}

Peripheral nerve injuries cause pain, disability, and economic burden in many affected individuals [1]. Each year around 300,000 people of working age in Europe suffer a peripheral nerve injury, and less than half of patients regain full function after treatment [2]. To date, the gold standard to repair nerve injury where nerve gap is larger than approximate $3 \mathrm{~cm}$ is autologous nerve grafting. However, this technique shows some major drawbacks, including limited supply of donor nerves, requiring an extra surgery, and sacrificial a healthy nerve [1, 3-5]. In recent years, advances have been made to engineer artificial nerve guidance conduits composed of synthetic, natural or composite polymers systems [6, 7]. Nerve guide conduits could be manufactured via numerous approaches, such as extrusion, solvent casting, 3D printing, electrospinning, and phase separation [8]. Among these approaches, electrospinning shows superior advantage in terms of producing micro/nanofibers. As a simple and low-cost technique, electrospinning offers the ability to use a wide range of biomaterials, including 
natural and synthetic polymers. For example, silk, gelatin, poly (caprolactone) (PCL), poly (lactic acid) (PLA), and poly (lactic-co-glycolic acid) (PLGA) have been electrospun to fibrous scaffold for nerve regeneration [9-11]. Moreover, electrospun scaffolds have large surface-tovolume ratio and their fibrous structure resemble native extracellular matrix [12].

Poly (trimethylene carbonate) (PTMC) is a biocompatible rubber-like polymer which has been shown to degrade by surface erosion without formation of acidic products [13]. For this reason, such elastomeric polymer has been evaluated as scaffolding materials for regenerative medicine and tissue engineering application [14-16]. To date, little work is reported about the fabrication of PTMC fibers using electrospinning technique. Reduced graphene oxide (rGO) and its family have drawn enormous attention in recent years due to its many interesting properties such as high charge carrier mobility, large theoretical specific surface area and excellent mechanical properties [17]. It has been demonstrated that electrically conductive surface could promote neuron cell growing, differentiation and signaling [18]. In this context, rGO has been extensively integrated with various polymers as scaffolding materials for nerve tissue engineering [19, 20]. For example, Aznar-Cervantes used silk fibroin/rGO composite to prepare conductive fibrous scaffold for promoting PC12 cells [21]. In our previous study, PTMC/rGO composite film was prepared by casting and showed a potential application in nerve regeneration [22]. However, $\mathrm{rGO} /$ polymer composites tend to become inhomogeneous as the concentration of rGO increases leading to a sacrificial performance. This problem could be solved by using functionalized graphene which would significantly reduce the enthalpic interaction [23]. It has been demonstrated that polymer-functionalized graphene could improve the performance of polymer/graphene composites [24, 25].

In the present work, a biocompatible and flexible fibrous scaffold was fabricated by electrospinning of a dispersion of PTMC and rGO-graft-PTMC. rGO-graft-PTMC was first prepared by grafting trimethylene carbonate (TMC) oligomers onto rGO. The benefits of such functionalization were in three ways. Firstly, it was expected to increase the loading amount of rGO into electrospun polymer fiber matrix. Secondly, it was carried out in an attempt to improve the interfacial adhesion with the PTMC fiber matrix. Thirdly, rGO fraction was present in composite fibers without an extra post-reduction process. The properties, such as hydrophilicity, fiber dimension, and fiber morphology of PTMC/rGO-graft-PTMC mats were characterized, and the cytocompatibility of PC12 cell line was investigated. 


\section{Materials and methods}

\subsection{Materials}

TMC Monomer was kindly provided by Huizhou Foryou Medical Devices. Sulfuric acid $\left(\mathrm{H}_{2} \mathrm{SO}_{4}\right)$, hydrochloric acid $(\mathrm{HCl})$ solution, phosphorus pentoxide, ammonium persulfate, potassium permanganate, hydrogen peroxide solution, 2-chloroethanol, sodium azide, Tin(II)2-ethylhexanoate $\left(\mathrm{Sn}(\mathrm{Oct})_{2}\right)$, and magnesium sulfate $\left(\mathrm{MgSO}_{4}\right)$ were purchased from Sigma Aldrich, The Nederlands. Graphite flake (natural, -325 mesh) was acquired from Alfa Aesar, Germany. Diethyl ether, acetone, toluene, dimethylformamide (DMF), chloroform and dichloromethane (DCM) were bought from VWR Chemicals, Germany. N-methyl-pyrrolidone (NMP) was purchased from Merck, Germany. Homemade azido ethanol was synthesized by reacting 2-chloroethanol and sodium azide according to previous study [26]. All the reagents and chemicals were of analytical grade and used without further purification unless otherwise stated.

\subsection{Synthesis of PTMC and rGO-graft-PTMC}

High molecular weight linear PTMC was synthesized by ring opening polymerization TMC as described in our previous study [27]. Briefly, ring opening polymerization of TMC was performed in a bulk condition with $\mathrm{Sn}(\mathrm{Oct})_{2}$ as catalyst at $130{ }^{\circ} \mathrm{C}$ under argon atmosphere for 2 days. The molecular weight of PTMC was determined by gel permeation chromatography.

To synthesize rGO-graft-PTMC, rGO initiator was firstly prepared using a nitrene chemistry method [28]. Briefly, GO (0.5 g) was dispersed in NMP $(200 \mathrm{~mL})$ by sonication for $2 \mathrm{~h}$ followed by addition of azido ethanol $(10 \mathrm{~g})$. GO was then thermal reduced into rGO and reacted with azido ethanol at $160^{\circ} \mathrm{C}$ under argon atmosphere. The product of rGO initiator was obtained by purifying the reaction mixture with acetone and subsequently dried in vacuum oven at room temperature for further use. rGO-graft-PTMC was synthesized by ring opening polymerization of TMC $(5 \mathrm{~g})$ in toluene $(15 \mathrm{~mL})$ with $\mathrm{rGO}$ initiator $(0.05 \mathrm{~g})$ under argon atmosphere. $\mathrm{Sn}(\mathrm{Oct})_{2}(0.02 \mathrm{~mol} / \mathrm{g}$ monomer $)$ was used as a catalyst and reaction was performed at $110{ }^{\circ} \mathrm{C}$ for 4 days. After cooling to room temperature, the sedimentation in the reaction flask was a mixture of homo PTMC and rGO-graft-PTMC. Toluene was removed, and the mixture was redispersed in DCM followed by centrifugation at $5000 \mathrm{rpm}$ for $10 \mathrm{~min}$ to remove homo 
PTMC. This purification step was repeated for 5 times. The final rGO-graft-PTMC was dried in vacuum oven at room temperature.

\subsection{Fourier transform infrared (FTIR) and thermogravimetric analysis (TGA)}

FTIR spectroscopy (PerkinElmer, Spectrum Two, UK) was performed to characterize the chemical structure of PTMC, GO, rGO initiator, and rGO-graft-PTMC. TGA was used to characterize thermal stability and graphene loading content of PTMC/rGO-graft-PTMC composite fibers. The measurements were examined over a temperature range of $50{ }^{\circ} \mathrm{C}$ to $550{ }^{\circ} \mathrm{C}$ at a heating rate of $20{ }^{\circ} \mathrm{C} / \mathrm{min}$ and a nitrogen flow of $20 \mathrm{~mL} / \mathrm{min}$.

\subsection{Electrospinning}

PTMC $(2.5 \%, \mathrm{w} / \mathrm{v})$ was dissolved in chloroform/DMF $(\mathrm{v} / \mathrm{v}=9 / 1)$ and used for electrospinning to obtain PTMC fibers. To prepare PTMC/rGO-graft-PTMC fibers, a dispersion of rGO-graftPTMC was firstly prepared by dispersing of rGO-graft-PTMC into a chloroform/DMF $(\mathrm{v} / \mathrm{v}=9 / 1)$ solvent mixture with ultrasonication for $2 \mathrm{~h}$. At mean time, a PTMC solution was prepared as mentioned above. PTMC solution and rGO-graft-PTMC dispersion of different proportions were then mixed to make mixtures with different weight ratios of rGO-graft-PTMC to PTMC, and the final concentration of PTMC in mixtures was kept constant at $2.5 \%(\mathrm{w} / \mathrm{v})$. The resultant mixtures were stirred for $24 \mathrm{~h}$ to obtain homogenous dispersion before electrospinning. For electrospinning, PTMC or PTMC/rGO-graft-PTMC solutions were placed into a $5 \mathrm{~mL}$ standard syringe equipped with a $27 \mathrm{G}$ blunted stainless steel needle using a syringe pump at a rate of $1.0 \mathrm{~mL} / \mathrm{h}$ with an applied voltage of $15 \mathrm{kV}$. The working distance from the tip of spinneret to aluminum plate collector was set at $20 \mathrm{~cm}$.

\subsection{Scanning electron microscopy (SEM) imaging}

Surface morphology of electrospun scaffolds was examined by secondary electron SEM (JSMIT100, JEOL). Prior to SEM imaging, samples were gold sputtered with a Cressington Sputter Coater 108 Auto set at $30 \mathrm{~mA}$ for $60 \mathrm{~s}$. For backscattering electron SEM, electrospun fibers were directly deposited on a carbon-coated (100-A) copper microscope grid for $5 \mathrm{~s}$ and then observed by a Zeiss MERLIN HR-SEM. 


\subsection{Fiber diameter and porosity of scaffolds}

The average fiber diameter and fiber diameter distribution were determined by measuring at least 15 fibers and 50 segments in one SEM image, and five images were used for each scaffold using Adobe Photoshop CS4. Porosity of scaffolds were calculated using the following equation [12]:

$$
p=\left(1-\frac{\rho^{\prime}}{\rho_{\circ}}\right) \times 100 \%
$$

Where $p$ is the porosity of scaffold porosity, $\rho^{\prime}$ is the apparent density of scaffold, and $\rho^{\circ}$ is the bulk density of polymer materials.

\subsection{Contact angle measurements}

The wettability of electrospun scaffolds was investigated by sessile drop technique using an optical contact angle device (OCA15, Dataphysics, Germany). Sessile milli-Q water drop was deposited onto sample surface with a syringe, and the drop contour was fitted by the YoungLaplace method. At least 3 different static contact angle measurements were performed for each sample.

\subsection{Cell culture and Cell seeding}

PC12 cells $\left(\right.$ ATCC $^{\circledR}$ CRL-1721 ${ }^{\mathrm{TM}}$, Germany) were expanded in T-175 flask and cultured in complete culture medium, comprising RPMI-1640 medium (Sigma Aldrich), 10\% horse serum (Gibco), 5\% fetal bovine serum (Gibco), and $100 \mathrm{U} / \mathrm{mL}$ penicillin and $100 \mathrm{mg} / \mathrm{mL}$ streptomycin (Gibco). The culture medium was refreshed every two days, and cells were allowed to grow until the culture reached at approximately $80 \%$ confluence.

Electropsun mats were punched into discs of $1 \mathrm{~cm}$ diameter followed by washing with ethanol for 3 times. After washing, samples were placed in 48-well plates, and rubber O-rings were placed on the top of them to avoid floating samples. For sterilization, samples were immersed in $70 \%$ ethanol for $10 \mathrm{~min}$ and rinsed with PBS for 2 times. After that, samples were soaked in 
$0.1 \mathrm{wt} \%$ gelatin solution for $1 \mathrm{~h}$ before cell seeding. PC12 cells were seeded on electrospun samples at a density of 10000 cells $/ \mathrm{cm}^{2}$. Cell-scaffold constructs were cultured in complete culture medium in an incubator with $5 \% \mathrm{CO}_{2}$ humid atmosphere at $37{ }^{\circ} \mathrm{C}$ up to 7 days. Cell culture medium was refreshed every two days.

\subsection{Cell viability}

Cell viability was tested by using PrestoBlue ${ }^{\mathrm{TM}}$ Cell Viability Reagent (Thermal fisher). Briefly, at each time point, cell culture medium in sample plates was replaced with fresh cell culture medium containing $10 \%(\mathrm{~V} / \mathrm{V})$ PrestoBlue $^{\mathrm{TM}}$ reagent, and the sample plates were incubated at $37{ }^{\circ} \mathrm{C}$ for $1 \mathrm{~h}$ avoiding light. Fluorescence intensity was measured using a Tecan plate reader (560/590 $\mathrm{nm}$ excitation/emission).

\subsection{Statistical analysis}

All data points were expressed as mean \pm standard deviation. Statistical comparison was determined using GraphPad Prism 5.01 (GraphPad software, San Diego, USA) for windows. Two-way analysis of variance (ANOVA) was performed to compare cell viability. 


\section{Result and discussion}

\subsection{Preparation of PTMC and rGO-graft-PTMC}

A

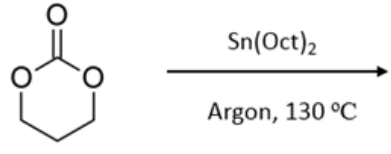

TMC

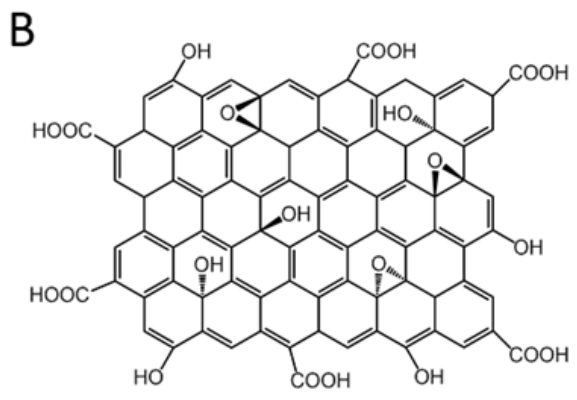

GO

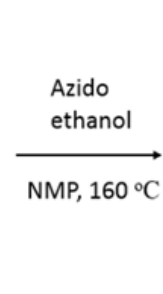

rGO initiator

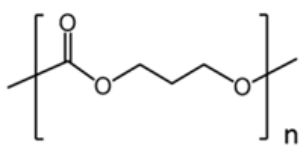

PTMC

Figure 1. Synthesis scheme of (A) PTMC and (B) rGO-graft-PTMC

High molecular weight PTMC was prepared by ring opening polymerization. The synthesis route and PTMC formula were shown in Figure 1A. The obtained PTMC had an average molecular weight of $500,000 \mathrm{~g} / \mathrm{mol}$. To synthesize rGO-graft-PTMC, rGO initiator with thermal stable hydroxyl groups was first prepared and subsequently used to initiate ringopening reaction of TMC as shown in Figure1B. The chemical structure of PTMC and rGOgraft-PTMC was verified by FTIR spectra (Figure 2). Stretching vibration of $-\mathrm{CH}_{2}-(2971$ and $\left.2909 \mathrm{~cm}^{-1}\right)$ and $\mathrm{C}=\mathrm{O}\left(1700 \mathrm{~cm}^{-1}\right)$ were observed in PTMC [29]. A broad band peak from 2900 to $3700 \mathrm{~cm}^{-1}$ were reflected by strong stretching of different hydroxyl groups in $\mathrm{GO}$, and $\mathrm{C}=\mathrm{C}$ stretching peak at $1635 \mathrm{~cm}^{-1}$ was also observed. The disappearance of unstable hydroxyl groups (2900-3700 $\mathrm{cm}^{-1}$ ) in rGO due to the reaction of azido ethanol with GO in NMP at high temperature. rGO-graft-PTMC had the stretching peaks at $1230 \mathrm{~cm}^{-1}, 1700 \mathrm{~cm}^{-1}$, and 2900 $\mathrm{cm}^{-1}$ which were assigned to the bands of $\mathrm{C}-\mathrm{O}, \mathrm{C}=\mathrm{O}$, and $\mathrm{C}-\mathrm{H}$ in PTMC, respectively. Taken together, these results demonstrated that GO was thermally reduced into rGO initiator, and PTMC was successfully grafted on rGO surface. 


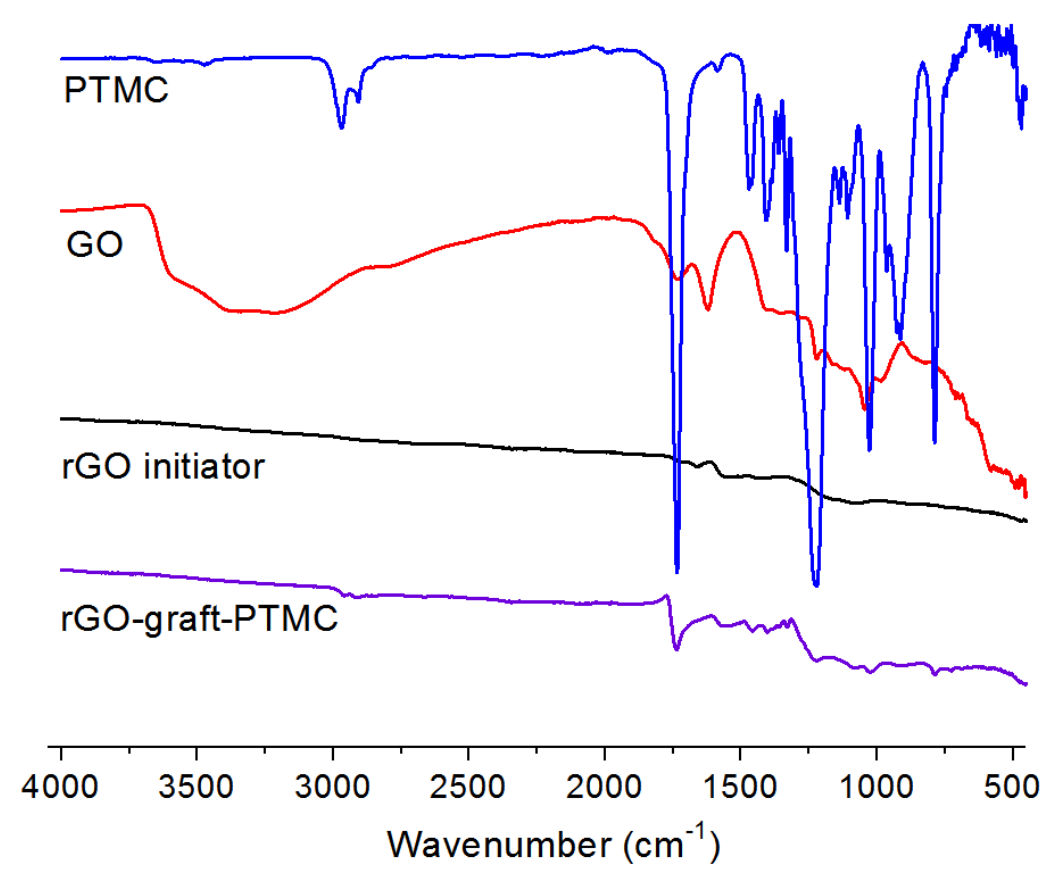

Figure 2. FTIR spectra of PTMC, GO, rGO initiator, and rGO-graft-PTMC.

\subsection{Fabrication of PTMC fibers and PTMC/rGO-graft-PTMC composite fibers}

rGO has been integrated with electrospun fiber matrices, such as silk, polyvinyl polyvinylpyrrolidone, and polyacrylonitrile [30,31]. However, an extra post-reduction process of GO is always required in these cases. In the present work, we fabricated rGO-based composite fibers without an extra post-reduction process via electrospinning of a dispersion of PTMC and rGO-graft-PTMC. Macroscopic view of PTMC/rGO-graft-PTMC composite fibers with various rGO-graft-PTMC content was shown in Figure 3. 


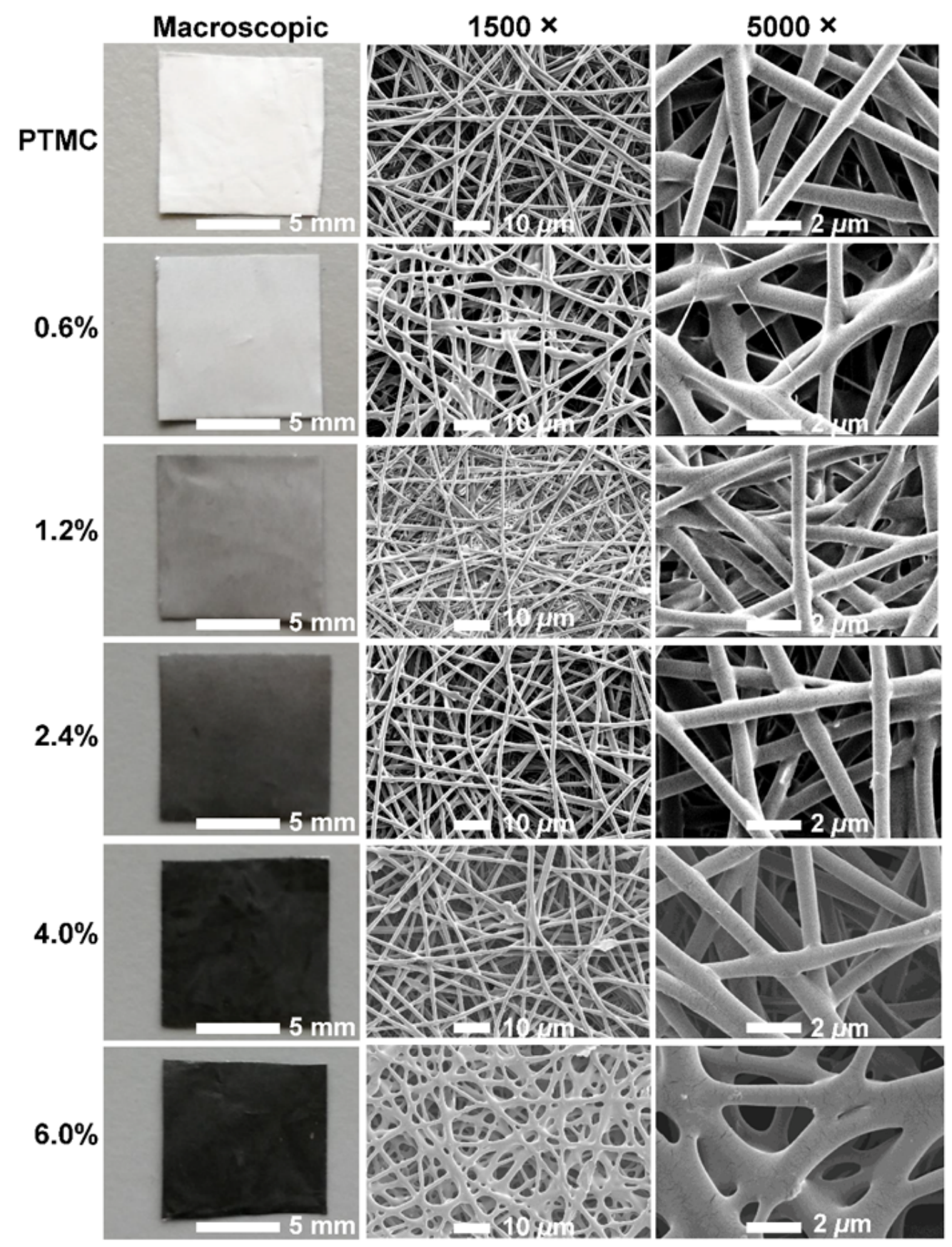

Figure 3. Macroscopic view and corresponding SEM images of PTMC fibers and PTMC/rGO-graftPTMC composite fibers with rGO-graft-PTMC weight percent of $0.6 \%, 1.2 \%, 2.4 \%, 4.0 \%$, and $6.0 \%$ relative to PTMC content. 

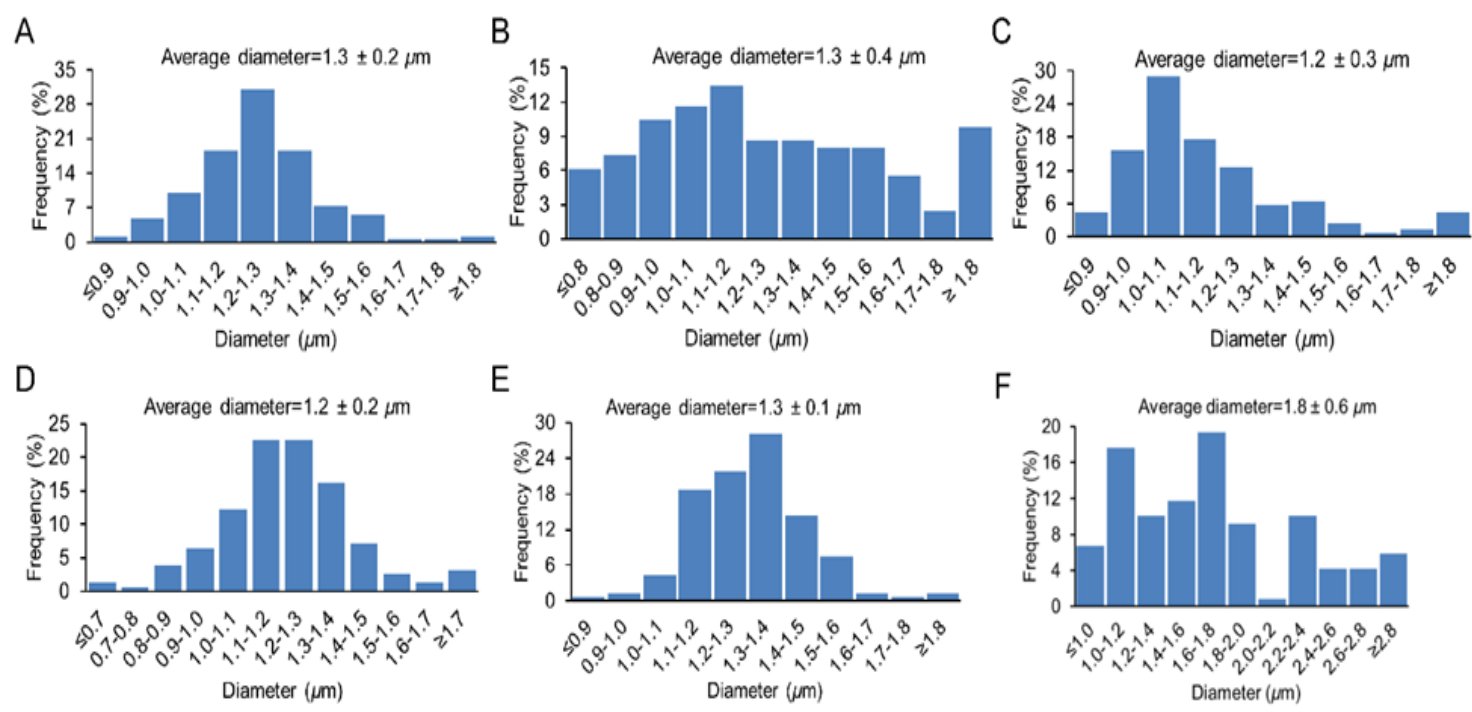

Figure 4. Distributions of fiber diameters of PTMC fibers (A) and PTMC/rGO-graft-PTMC composite fibers with rGO-graft-PTMC weight percent of (B) $0.6 \%$, (C) $1.2 \%$, (D) $2.4 \%$, (E) $4.0 \%$, and (F) $6.0 \%$ relative to PTMC content.

Pure PTMC fiber mats were milk white. After introduction of rGO-graft-PTMC, the fibrous mats became gray. As the concentration of rGO-graft-PTMC increased, the fibrous mats gradually turned black. Previous studies have demonstrated that the morphology of electrospun fibers is depended on various parameters such as working distance, applied voltage, flow rate, ambient humidity, and solution properties (eg. viscosity, polymer concentration, and surface tension) $[12,32]$. To know the influence of rGO-graft-PTMC content on fiber morphology, secondary electron SEM micrographs were acquired at different magnifications (Figure 3). The SEM micrographs showed a framework composed of randomly oriented fibers. When the portion of rGO-graft-PTMC increased from 0 to $4.0 \%$, homogeneous and smooth fiber morphology was observed. As the weight ratio reach up to $6.0 \%$, the fibers presented a heterogeneous morphology with an increase in fiber interconnection. Figure 4 shows the average fiber diameter and diameter distribution of electrospun mats. A similar average fiber diameter (around $1.3 \mu \mathrm{m}$ ) was detected with the concentration of rGO-graft-PTMC no higher than $4.0 \%$. When the weight ratio was further increased to $6.0 \%$, the average fiber diameter apparently increased $(1.8 \mu \mathrm{m})$ and the variations of fiber diameter became larger as well. The porosity of electrospun fiber mats was summarized in Table 1. Generally, the obtained electrospun mats had a highest porosity from $84 \pm 4 \%$ to $95 \pm 2 \%$. 
Table 1. The porosity of PTMC fibers and PTMC/rGO-graft-PTMC composite fibers with various rGO-graft-PTMC content.

\begin{tabular}{ll}
\hline Samples & porosity \\
\hline PTMC & $95 \pm 2 \%$ \\
\hline $\mathbf{0 . 6 \%}$ & $92 \pm 1 \%$ \\
\hline $\mathbf{1 . 2 \%}$ & $84 \pm 4 \%$ \\
\hline $\mathbf{2 . 4 \%}$ & $89 \pm 1 \%$ \\
\hline $\mathbf{4 . 0} \%$ & $93 \pm 1 \%$ \\
\hline $\mathbf{6 . 0} \%$ & $86 \pm 2 \%$ \\
\hline $\mathrm{N}=4$ &
\end{tabular}
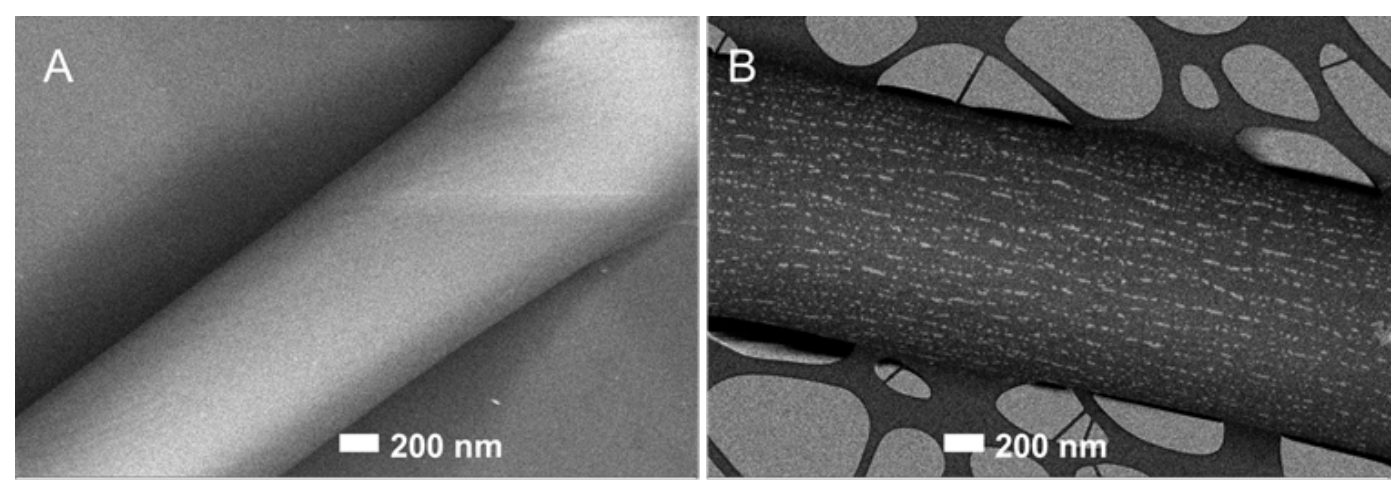

Figure 5. Backscattering images of fibers. (A) pure PTMC fibers. (B) rGO-graft-PTMC/PTMC fibers with 4.0 wt\% rGO-graft-PTMC relative to PTMC content.

Backscattering electron SEM imaging has been used to investigate phase morphology of polymer blends [33]. To investigate the presence of rGO-graft-PTMC in PTMC/rGO-graftPTMC composite fibers, backscattering electron SEM imaging was carried out for both PTMC and PTMC/rGO-graft-PTMC fibers. As shown in Figure 5A, the pure PTMC fibers present a smooth single-phase surface morphology. Bright spots on PTMC/rGO-graft-PTMC composite fibers were regarded as the rGO-graft-PTMC which was assembled within the fibers (Figure 5B). A similar finding was reported by Zhang et al. who assembled gold nanoparticles in poly (vinyl alcohol) (PVA) electrospun nanofibers. Their backscattering electron SEM imaging results demonstrated that bright spots in the PVA nanofibers were the gold nanoparticles [34]. 


\subsection{TGA analysis}

The thermal properties of rGO-graft-PTMC and electrospun fibers were characterized by TGA. The TGA curves were shown in Figure 6, and corresponding residual content at $500{ }^{\circ} \mathrm{C}$ was summarized in Table 2.

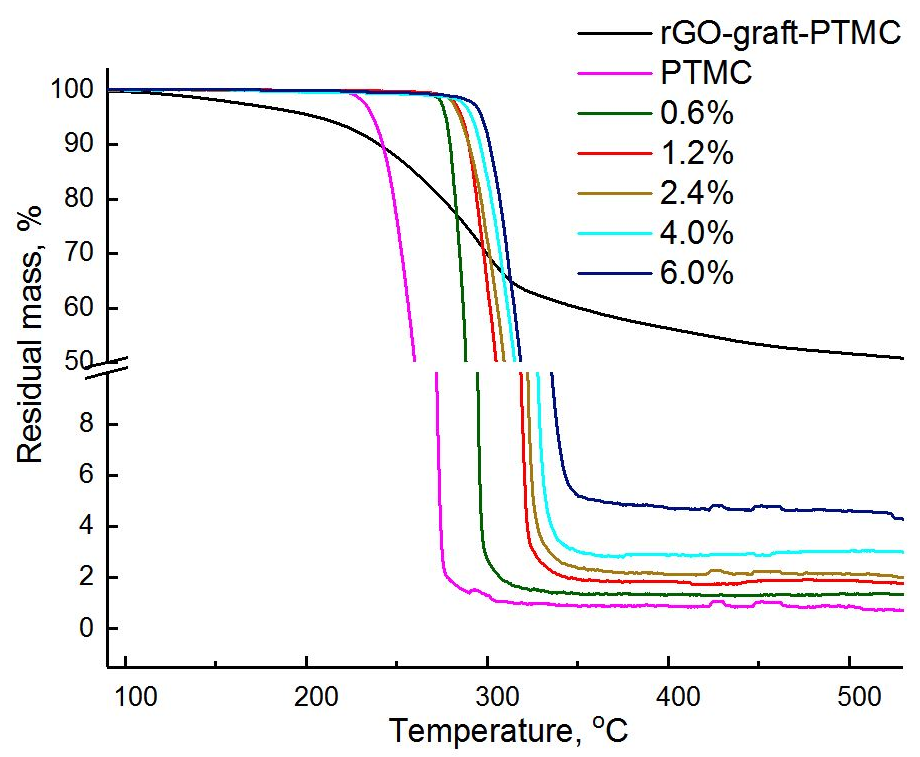

Figure 6. TGA curves of rGO-graft-PTMC, PTMC fibers, and PTMC/rGO-graft-PTMC composite fibers.

Table 2. TGA residual mass of $r G O$-graft-PTMC, PTMC fibers, and PTMC/rGO-graft-PTMC composite fibers.

\begin{tabular}{lc}
\hline Sample Code & Residual mass at $\mathbf{5 0 0}{ }^{\circ} \mathbf{C}, \mathbf{w t} \%$ \\
\hline rGO-graft-PTMC, $\mathbf{1 0 0 \%}$ & 51.6 \\
\hline PTMC & $0.86 \pm 0.36$ \\
\hline $\mathbf{0 . 6 \%}$ & $1.16 \pm 0.29$ \\
\hline $\mathbf{1 . 2} \%$ & $1.42 \pm 0.34$ \\
\hline $\mathbf{2 . 4} \%$ & $2.48 \pm 0.18$ \\
\hline $\mathbf{4 . 0} \%$ & $2.82 \pm 0.47$ \\
\hline $\mathbf{6 . 0} \%$ & $4.35 \pm 0.29$ \\
\hline
\end{tabular}

$\mathrm{N}=4$

rGO-graft-PTMC showed an obvious weight loss due to the grafted PTMC chains on the rGO surface. The degradation temperature of PTMC fibers was around $230^{\circ} \mathrm{C}$. The addition of rGO- 
graft-PTMC led to a modest increase in degradation temperature from $280{ }^{\circ} \mathrm{C}$ to $310{ }^{\circ} \mathrm{C}$ with increasing weight percent of rGO-graft-PTMC. The higher thermal stability of composite fibers attributed to the presence of rGO fractions which had a higher thermal stability than the polymer fiber matrix [35]. The rGO content in electrospun composite fibers could be estimated by the residual mass from TGA. As shown in Table 2, the percentage of residual mass for rGOgraft-PTMC was $51.09 \%$ which indicate the weight percent of rGO in rGO-graft-PTMC. PTMC fibers had residual mass percentage around $0.86 \pm 0.36 \%$. For the PTMC/rGO-graftPTMC composite fibers, the residual mass percentage increased with the increasing weight percent of rGO-graft-PTMC.

\subsection{Contact angle measurement}
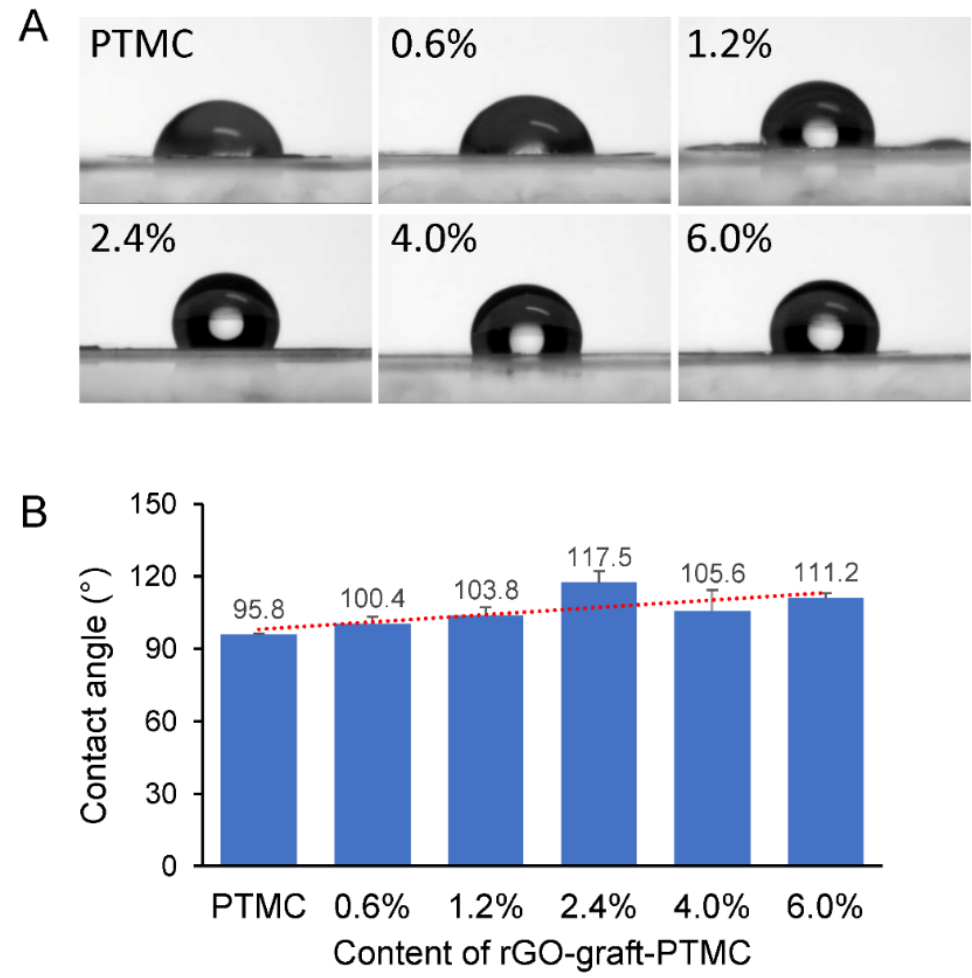

Figure 7. Contact angel measurement of PTMC fibers and PTMC/rGO-graft-PTMC composite fibers with various rGO-graft-PTMC content. (A) Representative images showed droplets on electrospun fibers. (B) The influence of rGO-graft-PTMC content on the wettability of electrospun fibers. 
The wettability of substrate has an important role in cellular proliferation, adhesion and differentiation [36]. Wettability of PTMC and PTMC/rGO-graft-PTMC electrsopun fibers were determined by contact angel measurement (Figure 7). Generally, all types of elctrospun fibers showed a hydrophobic surface leading to a long stay of water sessile droplet on their surface (Figure 7A). The incorporation of rGO-graft-PTMC resulted in slightly enhanced surface hydrophobicity due to the super hydrophobic property of rGO materials. The average contact angle value gradually increased from $95.8^{\circ}$ to $117.5^{\circ}$ when the portion of rGO-graftPTMC increased from 0 to $2.4 \%$, respectively (Figure $7 \mathrm{~B}$ ). After the threshold point of $2.4 \%$, the average contact angle value slightly dropped down even the amount of rGO-graft-PTMC reaching up to $6.0 \%$. Previous study has demonstrated that the contact angle of a surface is not only depended on the chemical components (surface energy), but also the geometric structure including surface roughness, fiber diameter and porosity [37, 38].

\subsection{Cell viability}

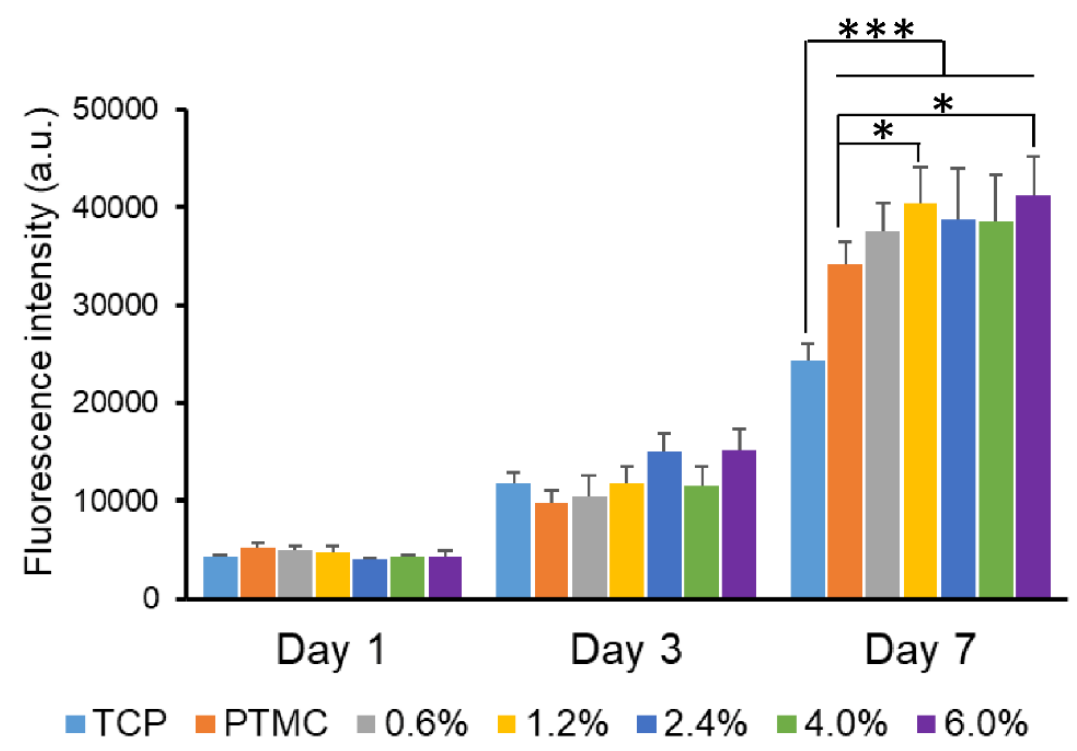

Figure 8. Biocompatibility of PTMC fibers and PTMC/rGO-graft-PTMC composite fibers with various rGO-graft-PTMC content.

PTMC is considered to be a biocompatible material and usually used as a scaffolding material for soft tissue regeneration [13]. Beside excellent physical properties, graphene materials 
promote cellular interaction due to their high surface area. However, graphene shows dosedependent cytotoxicity [17]. To investigate the biocompatibility of PTMC/rGO-graft-PTMC composite fibers, PC12 cells were seeded on fibrous mats for 7 days. PTMC fibers were used as control. The cell viability was investigated by PrestoBlue ${ }^{\mathrm{TM}}$ assay (Figure 8). Generally, the results revealed that cell numbers increased steadily for all kinds of fibers during the time course investigated. At day 1 and day 3, no statistical differences in cell viability were observed among all types of fibers regardless of rGO-graft-PTMC content. However, at day 7, PTMC/rGO-graft-PTMC composite fibers with 1.2 and $6.0 \mathrm{wt} \%$ of rGO-graft-PTMC showed significantly higher cell viability than that of pure PTMC fibers. Taken together, our results demonstrated that PTMC/rGO-graft-PTMC composite fibers showed good biocompatibility.

\section{Conclusion}

In the present study, PTMC/rGO-graft-PTMC composite fibers were successfully fabricated by using electrospinning technology. The loading of rGO-graft-PTMC was varied from 0 to $6.0 \mathrm{wt} \%$ relative to PTMC content. The fiber morphology and average diameter of PTMC/rGOgraft-PTMC composite fibers were affected by the content of rGO-graft-PTMC. In addition, the integration of rGO-graft-PTMC led to an increase in thermal stability and hydrophobicity. PC12 cell viability results demonstrated that PTMC/rGO-graft-PTMC composite fibers had good biocompatibility. Therefore, PTMC/rGO-graft-PTMC composite fibers were promising materials for nerve tissue regeneration.

\section{Acknowledgements}

The authors would like to thank HuiZhou Foryou Medical Company for kindly providing TMC monomer and the Chinese Scholarship Council for the financial support.

\section{References}

[1] X. Gu, F. Ding, D.F. Williams, Neural tissue engineering options for peripheral nerve regeneration, Biomaterials, 35 (2014) 6143-6156. 
[2] M. Georgiou, J.P. Golding, A.J. Loughlin, P.J. Kingham, J.B. Phillips, Engineered neural tissue with aligned, differentiated adipose-derived stem cells promotes peripheral nerve regeneration across a critical sized defect in rat sciatic nerve, Biomaterials, 37 (2015) 242-251.

[3] X. Jiang, S.H. Lim, H.-Q. Mao, S.Y. Chew, Current applications and future perspectives of artificial nerve conduits, Exp. Neurol., 223 (2010) 86-101.

[4] H. Millesi, Techniques for nerve grafting, Hand clinics viii., 16 (2000) 73-91,

[5] A. Faroni, S.A. Mobasseri, P.J. Kingham, A.J. Reid, Peripheral nerve regeneration: experimental strategies and future perspectives, Adv. Drug Deliv. Rev., 82 (2015) 160-167.

[6] S.H. Hsu, SH. Chan, C.T. Weng, S.H. Yang, C.F. Jiang, Long-term regeneration and functional recovery of a $15 \mathrm{~mm}$ critical nerve gap bridged by tremella fuciformis polysaccharide-immobilized polylactide conduits, Evid.-Based Complementary Altern. Med., 2013 (2013) 959261.

[7] T. Dinis, R. Elia, G. Vidal, Q. Dermigny, C. Denoeud, D. Kaplan, C. Egles, F. Marin, 3D multi-channel bi-functionalized silk electrospun conduits for peripheral nerve regeneration, $\mathrm{J}$. Mech. Behav. Biomed. Mater., 41 (2015) 43-55.

[8] M. Sarker, S. Naghieh, A.D. McInnes, D.J. Schreyer, X. Chen, Strategic design and fabrication of nerve guidance conduits for peripheral nerve regeneration, Biotechnol. J., (2018) 1700635.

[9] E.O. Johnson, P.N. Soucacos, Nerve repair: experimental and clinical evaluation of biodegradable artificial nerve guides, Injury, 39 (2008) 30-36.

[10] L. Ghasemi-Mobarakeh, M.P. Prabhakaran, M. Morshed, M.H. Nasr-Esfahani, S. Ramakrishna, Electrospun poly(ع-caprolactone)/gelatin nanofibrous scaffolds for nerve tissue engineering, Biomaterials, 29 (2008) 4532-4539.

[11] C.R. Wittmer, T. Claudepierre, M. Reber, P. Wiedemann, J.A. Garlick, D. Kaplan, C. Egles, Multifunctionalized electrospun silk fibers promote axon regeneration in the central nervous system, Adv. Func. Mater., 21 (2011) 4232-4242.

[12] H. Chen, X. Fan, J. Xia, P. Chen, X. Zhou, J. Huang, J. Yu, P. Gu, Electrospun chitosangraft-poly( $\varepsilon$-caprolactone)/poly( $\varepsilon$-caprolactone) nanofibrous scaffolds for retinal tissue engineering, Int. J. Nanomedicine, 6 (2011) 453. 
[13] K. Fukushima, Poly(trimethylene carbonate)-based polymers engineered for biodegradable functional biomaterials, Biomater. Sci., 4 (2016) 9-24.

[14] E. Bat, J. Feijen, D.W. Grijpma, Biodegradable elastomeric networks: highly efficient cross-linking of poly(trimethylene carbonate) by gamma irradiation in the presence of pentaerythritol triacrylate, Biomacromolecules, 11 (2010) 2692-2699.

[15] S. Sharifi, H.J.C. Kranenburg, B.P. Meij, D.W. Grijpma, Photo-crosslinkable poly (trimethylene carbonate)-based macromers for closure of ruptured intervertebral discs, Macromol. Symp., 309 (2011) 100-110.

[16] N. Zeng, A. van Leeuwen, H. Yuan, R.R. Bos, D.W. Grijpma, R. Kuijer, Evaluation of novel resorbable membranes for bone augmentation in a rat model, Clin. Oral Implants Res., 27 (2016) e8-e14.

[17] S. Goenka, V. Sant, S. Sant, Graphene-based nanomaterials for drug delivery and tissue engineering, J. Control. Release, 173 (2014) 75-88.

[18] N. Li, Q. Zhang, S. Gao, Q. Song, R. Huang, L. Wang, L. Liu, J. Dai, M. Tang, G. Cheng, Three-dimensional graphene foam as a biocompatible and conductive scaffold for neural stem cells, Sci. Rep., 3 (2013) 1604.

[19] C. Gardin, A. Piattelli, B. Zavan, Graphene in regenerative medicine: focus on stem cells and neuronal differentiation, Trends Biotechnol., 34 (2016) 435-437.

[20] N. Golafshan, M. Kharaziha, M. Fathi, Tough and conductive hybrid graphene-PVA: alginate fibrous scaffolds for engineering neural construct, Carbon, 111 (2017) 752-763.

[21] S. Aznar-Cervantes, A. Pagán, J.G. Martínez, A. Bernabeu-Esclapez, T.F. Otero, L. Meseguer-Olmo, J.I. Paredes, J.L. Cenis, Electrospun silk fibroin scaffolds coated with reduced graphene promote neurite outgrowth of PC-12 cells under electrical stimulation, Mater. Sci. Eng. C, 79 (2017) 315-325.

[22] S.G. Rotman, Z. Guo, D.W. Grijpma, A.A. Poot, Preparation and characterization of poly (trimethylene carbonate) and reduced graphene oxide composites for nerve regeneration, Polym. Adv. Technol., 28 (2017) 1233-1238.

[23] M. Fang, K. Wang, H. Lu, Y. Yang, S. Nutt, Covalent polymer functionalization of graphene nanosheets and mechanical properties of composites, J. Mater. Chem., 19 (2009) 7098-7105. 
[24] M. Fang, K. Wang, H. Lu, Y. Yang, S. Nutt, Single-layer graphene nanosheets with controlled grafting of polymer chains, J. Mater. Chem, 20 (2010) 1982-1992.

[25] Z. Xu, C. Gao, In situ polymerization approach to graphene-reinforced nylon-6 composites, Macromolecules, 43 (2010) 6716-6723.

[26] B.S. Sumerlin, N.V. Tsarevsky, G. Louche, R.Y. Lee, K. Matyjaszewski, Highly efficient "click" functionalization of poly (3-azidopropyl methacrylate) prepared by ATRP, Macromolecules, 38 (2005) 7540-7545.

[27] A.P. Pêgo, D.W. Grijpma, J. Feijen, Enhanced mechanical properties of 1, 3-trimethylene carbonate polymers and networks, Polymer, 44 (2003) 6495-6504.

[28] C. Gao, H. He, L. Zhou, X. Zheng, Y. Zhang, Scalable functional group engineering of carbon nanotubes by improved one-step nitrene chemistry, Chem. Mater., 21 (2008) 360-370.

[29] J. Han, C.J. Branford-White, L.M. Zhu, Preparation of poly( $(\varepsilon-$ caprolactone)/poly(trimethylene carbonate) blend nanofibers by electrospinning, Carbohydr. Polym., 79 (2010) 214-218.

[30] S. Aznar-Cervantes, J.G. Martínez, A. Bernabeu-Esclapez, A.A. Lozano-Perez, L. Meseguer-Olmo, T.F. Otero, J.L. Cenis, Fabrication of electrospun silk fibroin scaffolds coated with graphene oxide and reduced graphene for applications in biomedicine, Bioelectrochemistry, 108 (2016) 36-45.

[31] Y. Wang, J. Tang, S. Xie, J. Liu, Z. Xin, X. Liu, L.A. Belfiore, Leveling graphene sheets through electrospinning and their conductivity, RSC. Adv., 5 (2015) 42174-42177.

[32] J.M. Deitzel, J. Kleinmeyer, D. Harris, N.B. Tan, The effect of processing variables on the morphology of electrospun nanofibers and textiles, Polymer, 42 (2001) 261-272.

[33] G. Goizueta, T. Chiba, T. Inoue, Phase morphology of polymer blends: scanning electron microscope observation by backscattering from a microtomed and stained surface, Polymer, 33 (1992) 886-888.

[34] C.L. Zhang, KP. Lv, H.P. Cong, S.H. Yu, Controlled assemblies of gold nanorods in PVA nanofiber matrix as flexible free-standing SERS substrates by electrospinning, Small, 8 (2012) 648-653. 
[35] J.J. Mack, L.M. Viculis, A. Ali, R. Luoh, G. Yang, H.T. Hahn, F.K. Ko, R.B. Kaner, Graphite nanoplatelet reinforcement of electrospun polyacrylonitrile nanofibers, Adv. Mater., 17 (2005) 77-80.

[36] X. Zhu, W. Cui, X. Li, Y. Jin, Electrospun fibrous mats with high porosity as potential scaffolds for skin tissue engineering, Biomacromolecules, 9 (2008) 1795-1801.

[37] H. Chen, X. Huang, M. Zhang, F. Damanik, M.B. Baker, A. Leferink, H. Yuan, R. Truckenmüller, C. van Blitterswijk, L. Moroni, Tailoring surface nanoroughness of electrospun scaffolds for skeletal tissue engineering, Acta biomater., 59 (2017) 82-93.

[38] I. Wimpenny, N. Ashammakhi, Y. Yang, Chondrogenic potential of electrospun nanofibres for cartilage tissue engineering, J. Tissue Eng. Regen. Med., 6 (2012) 536-549. 


\section{Chapter 7 - Leachable poly(trimethylene carbonate)/ $\mathrm{CaCO}_{3}$ composites for additive manufacturing of micro-porous vascular structures}

Zhengchao Guo, Dirk Grijpma and André Poot

Department of Biomaterials Science and Technology, University of Twente, Enschede, The Netherlands

Published

Z. Guo, D.W. Grijpma, A.A. Poot, Leachable Poly(trimethylene carbonate)/CaCO $\mathrm{CO}_{3}$ composites for additive manufacturing of micro-porous vascular structures, Materials., 13 (2020) 3435 . 


\begin{abstract}
The aim of this work was to fabricate micro-porous poly(trimethylene carbonate) (PTMC) vascular structures by stereolithography (SLA) for applications in tissue engineering and organ models. Leachable $\mathrm{CaCO}_{3}$ particles with an average size of $0.56 \mu \mathrm{m}$ were used as porogen. Composites of photo-crosslinkable PTMC and $\mathrm{CaCO}_{3}$ particles were cast on glass plates, crosslinked by UV treatment and leached in watery $\mathrm{HCl}$ solutions. In order to obtain interconnected pore structures, the $\mathrm{PTMC} / \mathrm{CaCO}_{3}$ composites had to contain at least $30 \mathrm{vol} \%$ $\mathrm{CaCO}_{3}$. Leached PTMC films had porosities ranging from $33-71 \%$ and a pore size around 0.5 $\mu \mathrm{m}$. The mechanical properties of the micro-porous PTMC films matched with those of natural blood vessels. Resins based on $\mathrm{PTMC} / \mathrm{CaCO}_{3}$ composites with 45 vol\% $\mathrm{CaCO}_{3}$ particles were formulated and successfully used to build vascular structures of various shapes and sizes by SLA. The intrinsic permeabilities of the micro-porous PTMC films and vascular structures were at least one order of magnitude higher than reported for the extracellular matrix, indicating no mass transfer limitations in the case of cell seeding.
\end{abstract}

\title{
1 Introduction
}

Fabrication of artificial vascular structures is not only needed for traditional tissue engineering applications, but for disease models on chip as well [1-5]. Large tissue engineering is regarded as a viable strategy for the regeneration of organs, which may provide a solution for the limited availability of donor organs for transplantation [6-8]. However, engineering of tissues remains a challenge, because the viability of seeded cells as well as in situ tissue formation are dependent on the presence of a vascular system [9-11]. Therefore, tissue engineering scaffolds as well as chip-based organ models have been developed both containing vascular structures, using 3D printing of sacrificial templates and 3D bioprinting [12-17].

Additive manufacturing (AM) allows for the preparation of designed tissue engineering scaffolds with optimal properties concerning porosity, pore interconnectivity, pore size and pore geometry. Of all AM techniques, stereolithography (SLA) is the most versatile and accurate method allowing structures to be built at a resolution of 10-150 $\mu \mathrm{m}[18,19]$. Although for many applications pores sizes in this range or somewhat larger are suitable for cell seeding, the presence of (sub)micron-sized pores in the scaffold struts is advantageous in view of prolonged nutritional supply throughout the scaffold after implantation. Likewise, the walls of 
an artificial vascular network need to be micro-porous for the delivery of nutrients to cells and removal of waste products. Current commercial SLA machines, however, are not able to build pores in the (sub)micron range.

Porosity in tissue engineering scaffolds can be efficiently created by means of particle leaching [20]. $\mathrm{NaCl}$ and sugar particles are generally used for relatively large pores, ranging from tens to hundreds of micrometers, whereas micro-pores have been formed by leaching $2 \mu \mathrm{m} \mathrm{ZnO}$ crystals or 5-15 $\mu \mathrm{m} \mathrm{NaF}$ particles [21]. We have recently shown that scaffolds for bone regeneration can be built by SLA using a polymer/nano-hydroxyapatite composite [22]. Likewise, it should be possible to build vascular structures by SLA using a polymer/leachable particle composite. In this study we used $\mathrm{CaCO} 3$ as a porogen, because of the uniform size of the particles which show little tendency to aggregate and can be easily leached.

Synthetic polymers are widely used to build structures by SLA for biomedical applications $[23,24]$. In previous work, we have used flexible poly(trimethylene carbonate) (PTMC) to prepare tubular scaffolds for vascular tissue engineering by dipcoating or molding $[25,26]$. PTMC is an amorphous rubber-like polymer, that degrades by surface erosion in vivo without the formation of acidic degradation products [27-29]. Because of these characteristics, which are lacking in other polymers like poly(lactic acid) and poly( $\varepsilon$-caprolactone), PTMC is a very suitable material for vascular tissue engineering. The mechanical properties and degradation rate of PTMC networks can be tuned by varying the crosslink density as well as by copolymerization with e.g. poly(lactic acid) and poly(e-caprolactone) [28-30]. In a previous study, we have built a micro-vascular network from PTMC by SLA [31]. The capillaries had an inner diameter of approximately $200 \mu \mathrm{m}$ and a wall thickness of $150 \mu \mathrm{m}$. The walls of the channels were non-porous, which would hamper the formation of tissue around the capillaries. Therefore, in the present study, we aimed to fabricate micro-porous PTMC vascular structures by SLA and subsequent particle leaching.

\section{Materials and Methods}

\subsection{Materials}

Trimethylene carbonate (TMC) monomer was kindly provided by Huizhou Foryou Medical Devices, China. 1,1,1-Tris(hydroxymethyl)propane, 2-Hydroxy-4'-(2-hydroxyethoxy)-2methyl- propiophenone (Irgacure 2959), Tin(II)-2-ethylhexanoate (Stannous octoate, Sn(Oct)2), 
hydroquinone, methacrylic anhydride and triethylamine were purchased from Sigma Aldrich, The Netherlands. Propylene carbonate was ordered from Merck Millipore, Germany. Ethyl(2,4,6-trimethyl-benzoyl)-phenylphosphinate (Omnirad TPO-L) was obtained from IGM Resins, The Netherlands. Orasol Orange G dye was ordered from CIBA Specialty Chemicals, Switzerland. Hydrochloric acid (37\% (w/w) in water), analytical grade chloroform, dichloromethane (DCM), ethanol, methanol and acetone were purchased from VWR Chemicals, Germany. $\mathrm{CaCO}_{3}$ was ordered from Alfa Aesar, USA.

\subsection{Synthesis and functionalization of three-armed PTMC}

Three-armed PTMC was synthesized by ring-opening polymerization of TMC in a three-neck flask under argon atmosphere at $130{ }^{\circ} \mathrm{C}$ for 3 days [22]. 1,1,1-Tris(hydroxymethyl)propane and $\mathrm{Sn}(\mathrm{Oct})_{2}$ were used as initiator and catalyst, respectively. As resins for printing contained propylene carbonate diluent, three-armed PTMC was used to increase the possibility for crosslinking. The obtained PTMC was dissolved in DCM, and hydroquinone, triethylamine and methacrylic anhydride (MA) were added [22]. This was reacted at room temperature (RT) under argon protection for 5 days in the dark. PTMC-MA was obtained by precipitation in cold ethanol and drying in a vacuum oven at RT in the dark. The molecular weight $\left(\mathrm{M}_{\mathrm{n}}\right)$ and degree of functionalization of PTMC-MA were determined by ${ }^{1} \mathrm{H}-\mathrm{NMR}$ spectroscopy using a Bruker Ascend 400/Avance III 400 MHz NMR spectrometer.

\subsection{Preparation and characterization of PTMC-MA/CaCO3 films}

PTMC-MA and TPO-L were dissolved in chloroform (1 g PTMC-MA/3 mL chloroform, $5 \mathrm{wt} \%$ TPO-L relative to PTMC-MA). Various amounts of $\mathrm{CaCO}_{3}$ particles were dispersed in chloroform by sonication for 20 min. PTMC-MA/TPO-L solutions and $\mathrm{CaCO}_{3}$ dispersions were fully mixed and cast on glass plates using a casting knife. Chloroform was slowly evaporated overnight in the dark, after which the films were heated to $60{ }^{\circ} \mathrm{C}$ for $1 \mathrm{~h}$ to fully evaporate remaining chloroform. The $\mathrm{PTMC} / \mathrm{CaCO}_{3}$ composite films, containing 30-60 vol\% $\mathrm{CaCO}_{3}$ particles in the polymer matrix, were photo-crosslinked for $30 \mathrm{~min}$ in a UV box at 365 $\mathrm{nm}$ wavelength and $8 \mathrm{~mW} / \mathrm{cm}^{2}$ light intensity. To remove the sol fraction, the films were extracted for $3 \mathrm{~d}$ in chloroform, which was refreshed once per day. Finally, the composite films were immersed in ethanol and dried in a vacuum oven at $40{ }^{\circ} \mathrm{C}$ until constant weight. 
The gel content of the photo-crosslinked $\mathrm{PTMC} / \mathrm{CaCO}_{3}$ composite films was determined by extraction of the sol fraction in chloroform as described above. The following equation was used,

Gel content $=\frac{\mathrm{m}_{\mathrm{dry}}}{\mathrm{m}_{\text {initial }}} \times 100 \%$

in which $\mathrm{m}_{\text {dry }}$ is the mass of a PTMC/CaCO 3 composite film after extraction and drying and $\mathrm{m}_{\text {initial }}$ is the mass of a photo-crosslinked composite film before extraction.

\subsection{Leaching of $\mathrm{CaCO}_{3}$ particles and characterization of micro-porous PTMC films}

Photo-crosslinked and extracted $\mathrm{PTMC} / \mathrm{CaCO}_{3}$ composite films were immersed for $4 \mathrm{~d}$ in $3.7 \%$ (w/w) $\mathrm{HCl}$ solution in water, which was refreshed once per day. This yielded micro-porous PTMC films, which were finally soaked in distilled water.

The porosity of the photo-crosslinked micro-porous PTMC films was determined gravimetrically according to the following equation,

Porosity $=\left[1-\frac{m_{\text {dry }}}{\mathrm{V} \times \rho_{\mathrm{PTMC}}}\right] \times 100 \%$

in which $\rho_{\text {PTMC }}=1.31 \mathrm{~g} / \mathrm{cm}^{3}, \mathrm{~m}_{\text {dry }}$ is the dry weight of a micro-porous PTMC film and $\mathrm{V}$ the film's bulk volume in either dry or hydrated state, yielding the porosity in dry or hydrated state, respectively.

\subsection{Water flux}

Circular samples with a diameter of $26 \mathrm{~mm}$ were punched from hydrated micro-porous PTMC films. The samples were fixed in an Amicon cell 8003 (Merck Millipore, Germany) with a filtration area of $0.9 \mathrm{~cm}^{2}$. MilliQ water was introduced onto the membranes at a pressure of 
$0.13,0.23$ or 0.33 bar. Upon reaching a stable water flow through the films, the permeating water mass was measured every $10 \mathrm{~s}$ for a minimum of $20 \mathrm{~min}$.

\subsection{Mechanical properties}

Samples with a length of $60 \mathrm{~mm}$ and a width of $5 \mathrm{~mm}$ were punched from the micro-porous PTMC films. The tensile properties of the films were determined in both hydrated and dry state using a Zwick Z020 tensile tester. The initial grip to grip separation was $30 \mathrm{~mm}$ and a pulling rate of $50 \mathrm{~mm} / \mathrm{min}$ was applied. The stiffness of the samples was determined from the slope of the stress-strain curve between 3 and $6 \%$ of strain.

\subsection{Additive manufacturing of micro-vascular structures using $\mathrm{PTMC} / \mathrm{CaCO}$ resin}

A dispersion of calculated amounts of $\mathrm{CaCO}_{3}$ and PTMC-MA in chloroform was homogenized and precipitated in cold ethanol to yield a composite that was dried in a vacuum oven at $40{ }^{\circ} \mathrm{C}$ to constant weight. Resins were prepared by homogenizing the composite in propylene carbonate and adding TPO-L photo-initiator and Orasol Orange G dye. The resin formulation is shown in Table 1. Structures were designed using Rhino 3D design software. PTMC structures containing 45 vol\% $\mathrm{CaCO}_{3}$ were printed using an Ember Autodesk digital light processing stereolithograph at a pixel resolution of 50x50 $\mu \mathrm{m}$ and a step height of $50 \mu \mathrm{m}$. Layers were sequentially photo-crosslinked by exposure for $11 \mathrm{~s}$ to light with a wavelength of $405 \mathrm{~nm}$ and an intensity of $20 \mathrm{~mW} / \mathrm{cm}^{2}$. Built structures were extracted for $4 \mathrm{~d}$ in chloroform/acetone (1:1 v/v) solution, which was refreshed once per day. Subsequently, the structures were dried and immersed for $1 \mathrm{~d}$ in chloroform containing 1\% (w/v) Irgacure 2959, dried and post-cured for $4 \mathrm{~h}$ in a UV cabinet at $254 \mathrm{~nm}$ and $10 \mathrm{~mW} / \mathrm{cm}^{2}$. The $\mathrm{CaCO}_{3}$ particles were leached for $3 \mathrm{~d}$ in $3.7 \%(\mathrm{w} / \mathrm{w}) \mathrm{HCl}$ solution in water, which was finally replaced by distilled water. 
Table 1. Resin formulation for stereolithography.

\begin{tabular}{lcc}
\hline Component & Weight $(\mathrm{g})$ & Content $(\boldsymbol{\%})$ \\
\hline PTMC-MA & 46.5 & 22.1 \\
\hline CaCO $_{3}$ & 78.7 & 37.4 \\
\hline Propylene carbonate & 85.0 & 40.5 \\
\hline TPO-L & 2.3 & $5^{*}$ \\
\hline Orasol Orange G & 0.07 & $0.15^{*}$ \\
\hline
\end{tabular}

*relative to the mass of PTMC-MA

\subsection{Scanning electron microscopy (SEM) and thermogravimetric analysis (TGA)}

Samples were sputtered with gold using a Cressington Sputter Coater 108 Auto set at $40 \mathrm{~mA}$ for $60 \mathrm{~s}$. The average size of the $\mathrm{CaCO}_{3}$ particles was determined by measuring the size of 500 particles by SEM (JSM-IT100, JEOL, Japan). Surfaces and cross-sections of PTMC/CaCO 3 composite films and printed micro-vascular structures, both before and after leaching of the $\mathrm{CaCO}_{3}$ particles, were also observed by SEM. $\mathrm{CaCO}_{3}$ content of the printed $\mathrm{PTMC} / \mathrm{CaCO}_{3}$ micro-vascular structures was determined by TGA. The measurements were carried out using a temperature range of $50-550{ }^{\circ} \mathrm{C}$ at a heating rate of $20{ }^{\circ} \mathrm{C} / \mathrm{min}$ and a nitrogen flow of 20 $\mathrm{mL} / \mathrm{min}$ (PerkinElmer, Pyris 1, UK).

\section{Results and Discussion}

\subsection{Characterization of PTMC-MA, CaCO 3 particles and PTMC-MA/CaCO 3 composite films}

The synthesized PTMC-MA had a molecular weight $\left(\mathrm{M}_{\mathrm{n}}\right)$ of $4,500 \mathrm{~g} / \mathrm{mol}$ and degree of functionalization with methacrylate groups of $97 \%$.

As shown in Figure 1, the $\mathrm{CaCO}_{3}$ particle size distribution ranged from 0.1 to $3.6 \mu \mathrm{m}$. The average particle size was $0.56 \pm 0.32 \mu \mathrm{m}$, which indicated the potential of the $\mathrm{CaCO}_{3}$ particles as leachable component given the intended SLA layer thickness of $50 \mu \mathrm{m}$. 


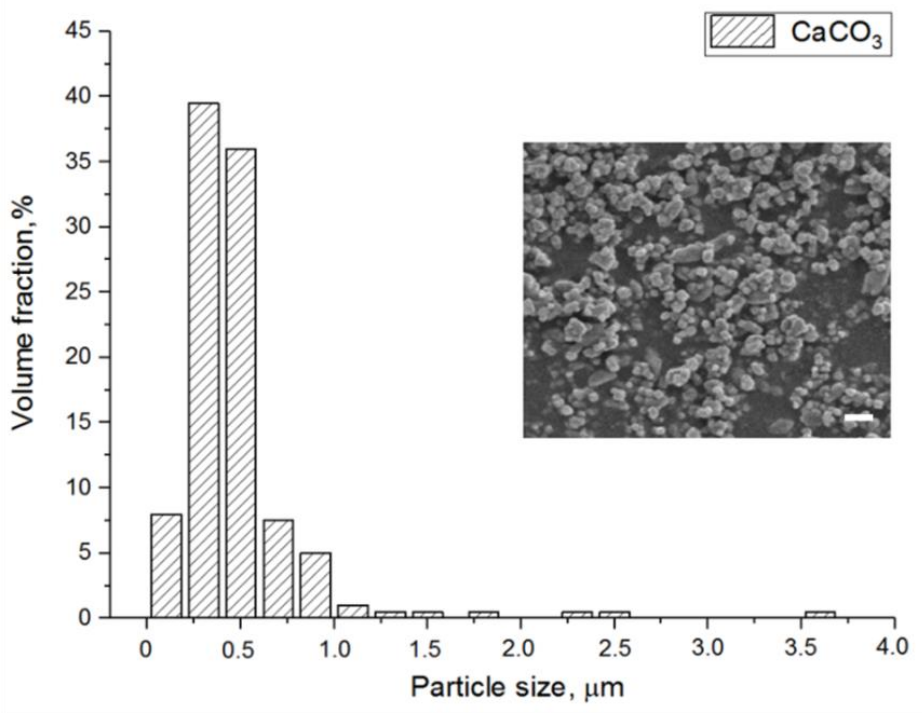

Figure 1. $\mathrm{CaCO}_{3}$ particle size distribution and SEM image of the particles. Scale bar $1 \mu \mathrm{m}$.

To be able to leach all $\mathrm{CaCO}_{3}$ particles, the porous structure formed upon leaching should have interconnected pores. Therefore, a percolation threshold study was carried out, by preparing PTMC/CaCO 3 composite films with $30,40,50$ or 60 vol\% $\mathrm{CaCO}_{3}$ particles in the polymer matrix. As shown in Table 2, the composite films had a gel content of at least 94.9\%, which increased with decreasing amount of $\mathrm{CaCO}_{3}$ particles. Pure PTMC films without $\mathrm{CaCO}_{3}$ had the highest gel content of $98.1 \%$. Thus, although the presence of the particles slightly decreased UV crosslinking efficiency, the high gel contents indicated the formation of stable $\mathrm{PTMC} / \mathrm{CaCO}_{3}$ composite films.

Table 2. Gel contents of PTMC and PTMC/CaCO 3 composite films.

\begin{tabular}{lll}
\hline Sample code & $\mathbf{C a C O}_{\mathbf{3}}$ loading, vol\% & Gel content, $\%$ \\
\hline PTMC & 0 & $98.1 \pm 0.3$ \\
\hline PTMC/30 & 30 & $97.2 \pm 1.1$ \\
\hline PTMC/40 & 40 & $97.4 \pm 0.7$ \\
\hline PTMC/50 & 50 & $96.6 \pm 0.6$ \\
\hline PTMC/60 & 60 & $94.9 \pm 2.1$ \\
\hline
\end{tabular}

All measurements $\mathrm{N}=4$. 
Figure 2 shows SEM images of cross-sections of the PTMC/CaCO 3 composite films. With increasing $\mathrm{CaCO}_{3}$ loading more particles were observed, which were homogeneously distributed.

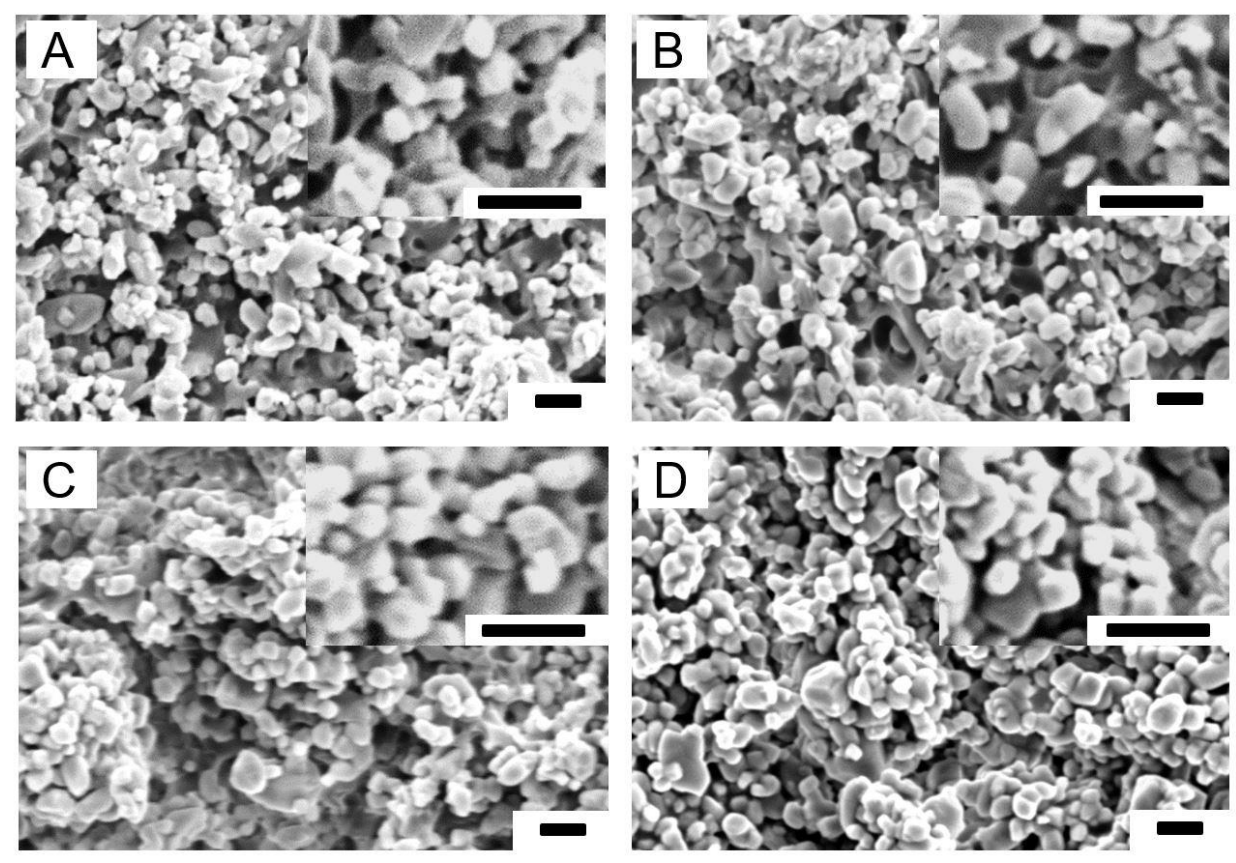

Figure 2. SEM pictures of cross-sections of PTMC/CaCO 3 composite films. (A) PTMC/30; (B) PTMC/40; (C) PTMC/50; (D) PTMC/60. Scale bar $1 \mu \mathrm{m}$. 


\subsection{Characterization of leached PTMC-MA/CaCO3 composite films}
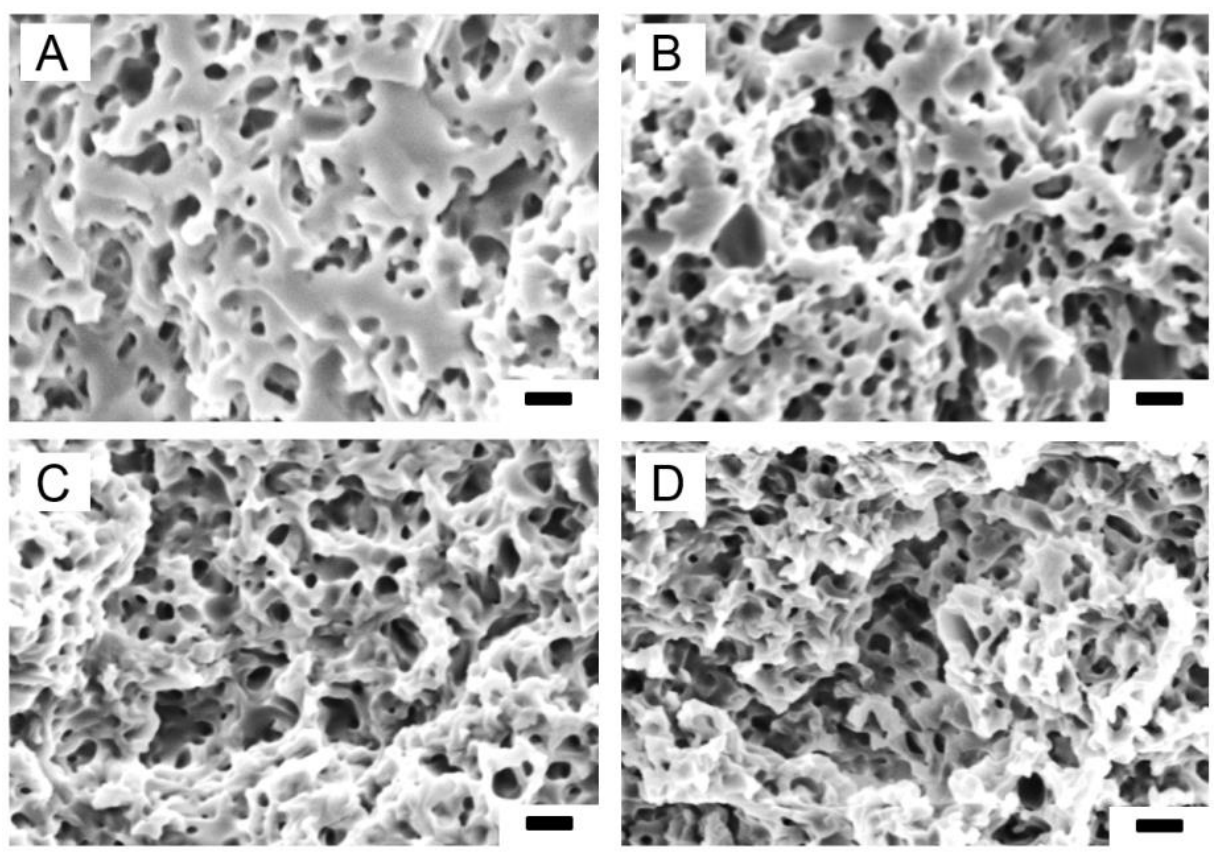

Figure 3. SEM pictures of cross-sections of leached PTMC/CaCO 3 composite films. (A) PTMC/30; (B) PTMC/40; (C) PTMC/50; (D) PTMC/60. Scale bar $1 \mu \mathrm{m}$.

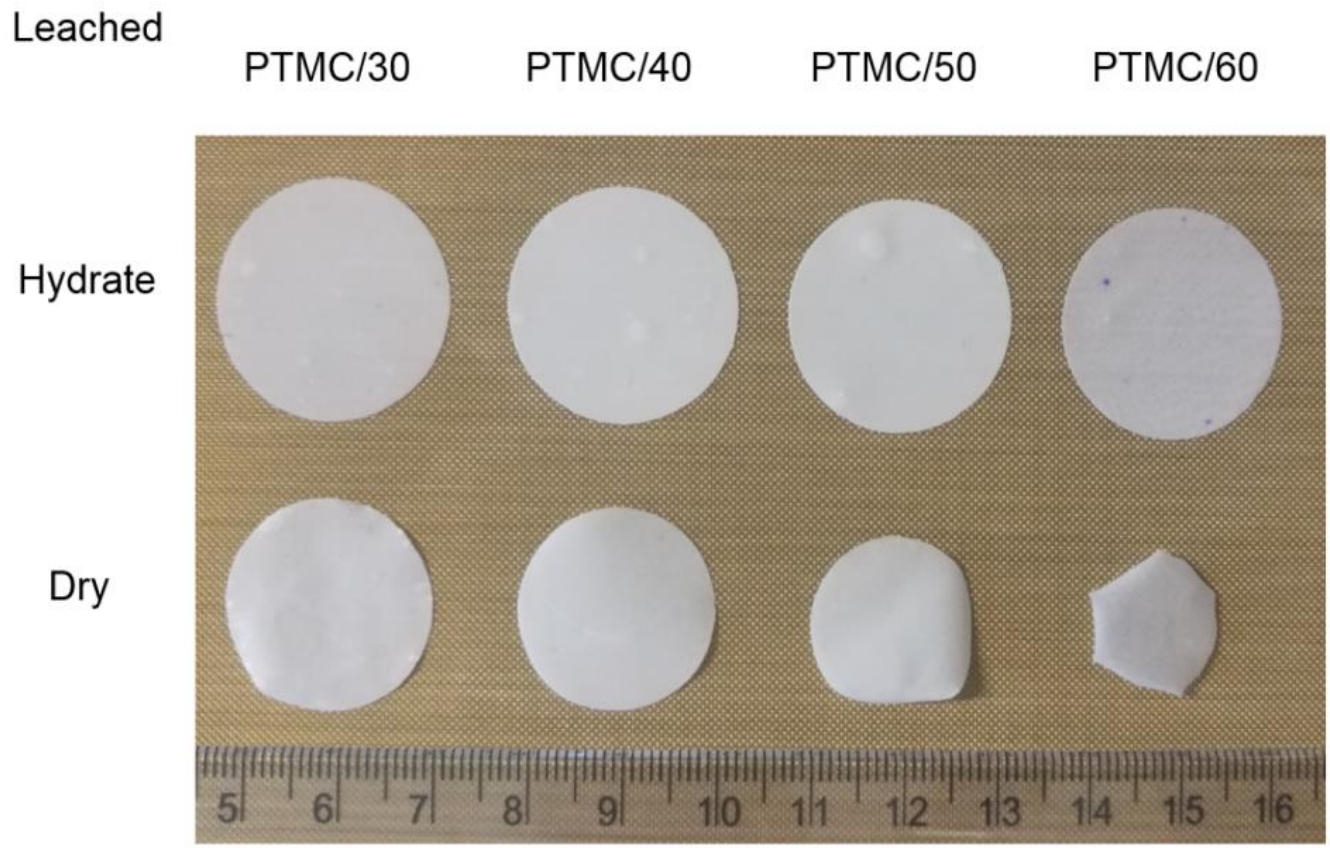

Figure 4. Macroscopic images of leached PTMC/CaCO 3 composite films in hydrated and dry state. 
All PTMC-MA/CaCO3 composite films were fully leachable in $3.7 \%(\mathrm{w} / \mathrm{w}) \mathrm{HCl}$ solution, see Figure 3. With increasing $\mathrm{CaCO}_{3}$ content more pores were observed, which were homogeneously distributed. The average pore sizes, determined from the SEM pictures, ranged from $0.45-0.50 \mu \mathrm{m}$ (Table 3). This is somewhat smaller than the average size of the $\mathrm{CaCO}_{3}$ particles $(0.56 \mu \mathrm{m})$. It should be noted that the pore sizes were determined in the dry state, which resulted in shrinkage of the leached films. This led to lower porosities of the films in the dry state as compared to the hydrated state, see Table 3. Moreover, the differences in porosities between dry and hydrated state increased with increasing $\mathrm{CaCO}_{3}$ content of the composite films. Thus, shrinkage of the dry films was higher at higher porosities, which is illustrated in Figure 4. Most probably, shrinkage of the leached films in the dry state was caused by the presence of pores, resulting in a relatively unstable structure, in combination with the low Tg of PTMC of around $-20{ }^{\circ} \mathrm{C}$. In the hydrated state, the pores were filled with water which stabilized the structure. The thickness of the leached films in the hydrated state was around $120 \mu \mathrm{m}$.

Table 3. Porosity and pore size of leached PTMC/CaCO 3 composite films.

\begin{tabular}{llll}
\hline Sample code & $\begin{array}{l}\text { Porosity, } \% \\
\text { Hydrated }\end{array}$ & $\begin{array}{l}\text { Porosity, } \% \\
\text { Dry }\end{array}$ & Pore size, $\boldsymbol{\mu m}$ \\
\hline PTMC/30 & $33.2 \pm 1.9$ & $31.4 \pm 2.2$ & $0.49 \pm 0.27$ \\
\hline PTMC/40 & $43.1 \pm 2.4$ & $41.9 \pm 1.4$ & $0.50 \pm 0.21$ \\
\hline PTMC/50 & $57.3 \pm 3.7$ & $52.1 \pm 4.3$ & $0.45 \pm 0.36$ \\
\hline PTMC/60 & $71.7 \pm 5.1$ & $51.3 \pm 3.9$ & $0.46 \pm 0.29$ \\
\hline
\end{tabular}

All measurements $\mathrm{N}=4$, except for pore size $\mathrm{N}=75$.

\subsection{Water permeability of the micro-porous PTMC films}

For the delivery of nutrients to cells and removal of waste products, tissue engineering scaffolds should not only be porous but also permeable to watery solutions. This was tested for the microporous PTMC films by water flux measurements, see Figure 5. Except the leached composite films with 30 vol\% $\mathrm{CaCO}_{3}$, all other PTMC films showed a water flux through the microporous structures at pressures up to 0.33 bar. Water flux increased with increasing porosity of the films. Although the PTMC/30 composite films could be fully leached, indicating 
interconnected pores, a pressure of 0.33 bar in conjunction with the relatively low porosity of $33.2 \%$ was apparently not high enough to induce a flow of water through the films.

A water pressure of 0.16 bar is a physiological pressure corresponding to $120 \mathrm{~mm} \mathrm{Hg}$. The extrapolated water flux at 0.16 bar was used to calculate the intrinsic permeability of the PTMC/40, PTMC/50 and PTMC/60 micro-porous films according to Darcy's formula [32]. The corresponding values were $3.13 \cdot 10^{-17}, 6.25 \cdot 10^{-15}$ and $1.88 \cdot 10^{-14} \mathrm{~m}^{2}$, respectively. This is at least 10 -fold higher than the intrinsic permeability of $1.32 \cdot 10^{-18} \mathrm{~m}^{2}$ reported for the extracellular matrix [33], indicating that these micro-porous structures would not hamper the delivery of nutrients to cells and removal of waste products.

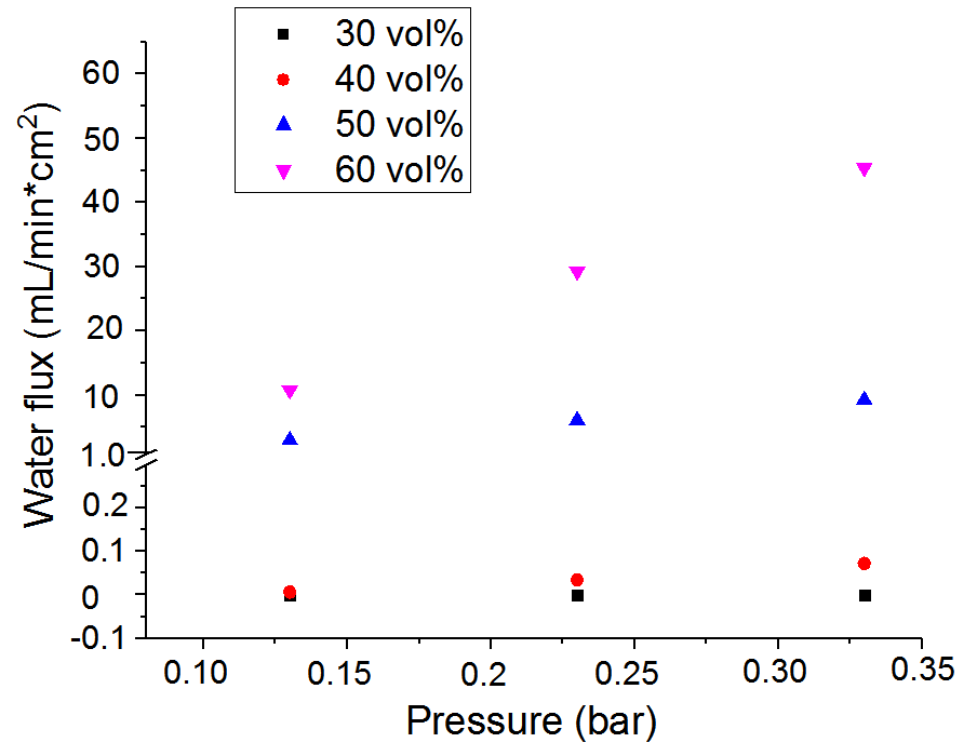

Figure 5. Water flux through the micro-porous PTMC films, vol\% refers to the amount of $\mathrm{CaCO}_{3}$ particles used during preparation of the films.

\subsection{Mechanical properties of the micro-porous PTMC films}

The mechanical properties of PTMC and micro-porous PTMC films are shown in Table 4. In the hydrated state, both stiffness $\left(E_{\bmod }\right)$ and maximum strength $\left(F_{\max }\right)$ of the micro-porous films decreased with increasing porosity. Compared to dense PTMC films, the micro-porous films had lower $\mathrm{E}_{\bmod }$ and $\mathrm{F}_{\max }$ and higher elongation at break due to the presence of pores. The same was observed for films in the dry state, albeit that $E_{\bmod }$ and $F_{\max }$ decreased much less with increasing porosity due to the shrinkage of the micro-porous films upon drying. 
The leached PTMC/50 films in the hydrated state had similar $\mathrm{E}_{\bmod }, \mathrm{F}_{\max }$ and elongation at break as native blood vessels [25], indicating the suitability of these structures for cardiovascular applications.

Table 4. Mechanical properties of PTMC and micro-porous PTMC films in hydrated and dry conditions.

\begin{tabular}{lllllll}
\hline & \multicolumn{5}{c}{ Hydrated } & \multicolumn{3}{c}{ Dry } & \\
\hline \multirow{2}{*}{$\begin{array}{l}\text { Sample } \\
\text { code }\end{array}$} & $\mathbf{E}_{\text {mod, }}$ & $\mathbf{F}_{\text {max }}$, & $\begin{array}{l}\text { Elongation at } \\
\text { break, } \%\end{array}$ & $\begin{array}{l}\mathbf{E}_{\text {mod, }}, \\
\mathbf{M P a}\end{array}$ & $\begin{array}{l}\mathbf{F}_{\text {max }}, \\
\mathbf{M P a}\end{array}$ & $\begin{array}{l}\text { Elongation at } \\
\text { break, } \%\end{array}$ \\
\hline PTMC & $8.16 \pm 0.43$ & $4.81 \pm 0.89$ & $67.1 \pm 10.9$ & $9.07 \pm 0.34$ & $6.57 \pm 0.14$ & $83.6 \pm 4.2$ \\
\cline { 2 - 7 } PTMC/30 & $3.52 \pm 0.16$ & $3.28 \pm 0.72$ & $89.6 \pm 12.5$ & $7.76 \pm 0.15$ & $5.76 \pm 1.21$ & $121.7 \pm 12.1$ \\
\cline { 2 - 7 } PTMC/40 & $2.72 \pm 0.14$ & $2.79 \pm 0.52$ & $109.4 \pm 11.7$ & $6.95 \pm 0.17$ & $4.58 \pm 1.79$ & $131.5 \pm 14.2$ \\
\cline { 2 - 7 } PTMC/50 & $1.13 \pm 0.05$ & $1.48 \pm 0.21$ & $103.5 \pm 8.1$ & $5.82 \pm 0.18$ & $2.70 \pm 0.54$ & $143.3 \pm 24.1$ \\
\cline { 2 - 7 } PTMC/60 & $0.30 \pm 0.06$ & $0.54 \pm 0.04$ & $104.1 \pm 10.2$ & $6.13 \pm 0.16$ & $2.16 \pm 0.11$ & $86.8 \pm 19.9$ \\
\hline
\end{tabular}

All measurements $\mathrm{N}=4$.

\subsection{Structure design, resin formulation and SLA}

A branched vascular structure was designed as shown in Figure 6A. The resin for SLA-based printing contained PTMC, $\mathrm{CaCO}_{3}$, photo-initiator TPO-L and Orasol Orange $\mathrm{G}$ dye in propylene carbonate diluent. The composition of the resin is shown in Table 1 and a macroscopic image of the resin in Figure 6B. Based on the above percolation threshold study, 45 vol\% $\mathrm{CaCO}_{3}$ relative to the PTMC matrix was chosen as leachable component for the creation of a micro-porous structure. The Orasol Orange $\mathrm{G}$ dye content was optimized to ensure a proper curing depth as previously described [34]. Branched vascular structures were successfully built and extracted, see Figures 6D and E, respectively. As complete leaching of these structures was not possible due to pore collapse, a post-curing step was implemented after extraction. After printing in various shapes and sizes, post-cured structures could be fully leached as shown in Figures 6F-H. 

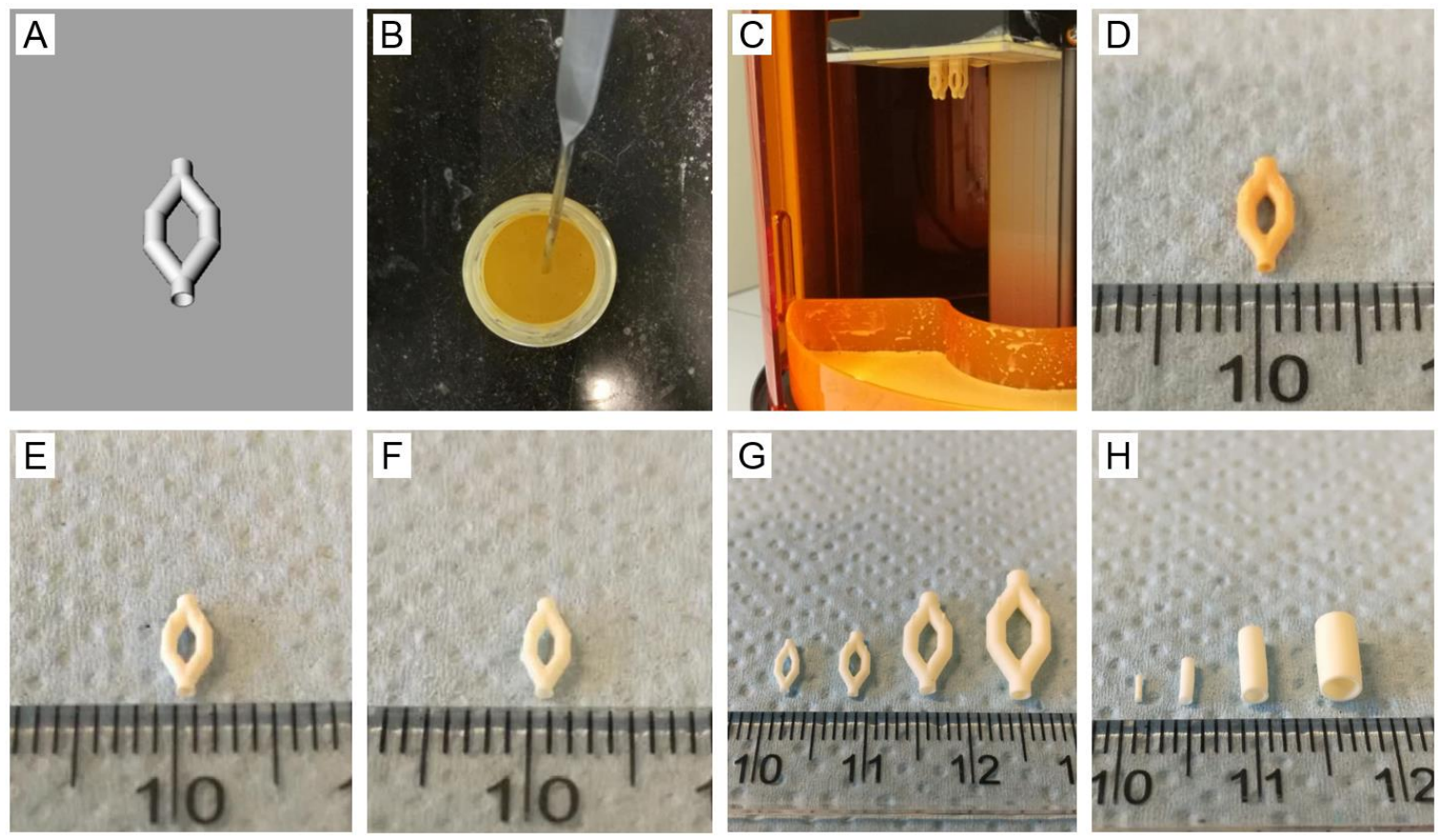

Figure 6. (A) design of branched vascular structure; (B) $\mathrm{PTMC/CaCO}$ composite resin; (C) built structures attached to printing head after SLA; (D) vascular structure before extraction; (E) vascular structure after extraction; $(F)$ vascular structure after extraction, post-curing and leaching (hydrated); $(G)$ branched vascular structures of different sizes (extracted, post-cured and leached, hydrated); $(H)$ vascular tubes of different sizes (extracted, post-cured and leached, hydrated).

\subsection{Characterization of the branched vascular PTMC structures}

SEM images of the vascular structures shown in Figures $6 \mathrm{E}$ and $\mathrm{F}$ are presented in Figure 7. Cross-sections showed open branched tubular structures, both in the case of non-leached and leached samples (Figures 7A and G, respectively). The outer surfaces clearly showed that the structures were built layer by layer, see Figures 7D, E, J and K. Cross-sections of non-leached samples showed $\mathrm{CaCO}_{3}$ particles, which were replaced by pores after leaching (Figures $7 \mathrm{C}, \mathrm{F}$ and I, L, respectively). 

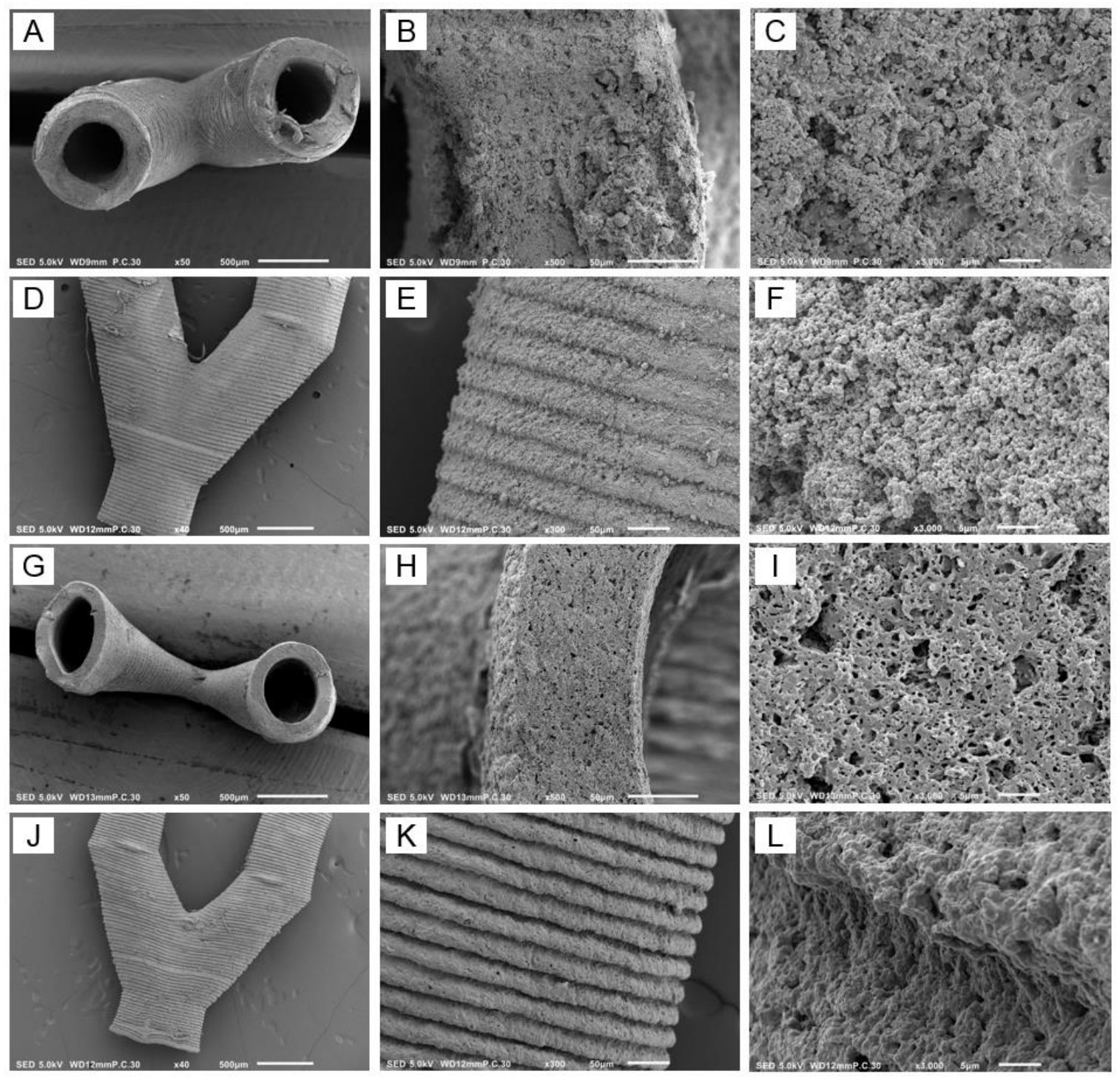

Figure 7. SEM images of (A-F) non-leached and (G-L) leached SLA-built vascular structures after extraction and post-curing. $A, B, C$ and $G, H, I$ show cross-sections, whereas $D, E, F$ and $J, K, L$ show the outer surfaces of the channels. Scale bar $500 \mu m(A, D, G, J)$, scale bar $50 \mu m(B, E, H, K)$, scale bar $5 \mu m(C, F, I, L)$.

As shown in Table 5, the leached vascular structures in the hydrated state had an inner diameter of $482 \mu \mathrm{m}$ and a wall thickness of $146 \mu \mathrm{m}$. Both values were lower in the dry state, caused by shrinkage of the structures similar as observed for the films. Taking shrinkage into account, pore sizes in the dry state around $0.40 \mu \mathrm{m}$ were in agreement with a mean particle size of 0.56 $\mu \mathrm{m}$. On SEM pictures (Figures 7I and L), some larger pores were visible probably formed by particle agglomerates. The $\mathrm{CaCO}_{3}$ content determined by TGA of the non-leached vascular structures was 50.2 vol\% (Table 5), which is higher than the theoretical value of 45 vol\%. This 
can be explained by extraction of non-crosslinked PTMC before post-curing. This was also observed for PTMC/nano-hydroxyapatite composite scaffolds fabricated by SLA [22]. The porosity of the leached vascular structures in the hydrated state was $59 \%$, which is in agreement with the $\mathrm{CaCO}_{3}$ particle content. Again because of shrinkage, the porosity of the structures in the dry state was lower (35\%). Based on the water flux through the walls of the vascular structures, as shown in Table 5, an intrinsic permeability of $0.61 \cdot 10^{-16} \mathrm{~m}^{2}$ was calculated. This is 50-fold higher than reported for the extracellular matrix, see discussion above for the films, indicating that the branched vascular structures will be highly permeable to nutrients and cellular waste products.

As our previous micro-vascular PTMC network built by SLA facilitated the adhesion and proliferation of human umbilical vein endothelial cells [31], a good biocompatibility of the vascular structures printed in the present study is expected as well. This is supported by other studies, in which PTMC scaffolds built by SLA were shown to be biocompatible with human mesenchymal stem cells and annulus fibrosus cells [22] [35] [36]. Both in vitro and in vivo, photo-crosslinked PTMC networks degrade by surface erosion. Degradation rate increases with increasing macromer molecular weight, i.e. decreasing crosslink density [28]. This also holds for networks prepared from linear PTMC crosslinked by $\gamma$-irradiation [37] [38].

Table 5. Parameters of SLA-printed vascular structures.

\begin{tabular}{llll}
\hline & Non-leached & Leached, hydrated & Leached, dry \\
\hline Inner diameter, $\boldsymbol{\mu m}$ & $480.2 \pm 10.9$ & $482 \pm 10.2$ & $416.3 \pm 7.5$ \\
\hline Wall thickness, $\boldsymbol{\mu m}$ & $162.5 \pm 4.3$ & $146.0 \pm 6.1$ & $90.1 \pm 3.8$ \\
\hline Pore size, $\boldsymbol{\mu m}$ & - & - & $0.40 \pm 0.27$ \\
\hline $\mathbf{C a C O}_{\mathbf{3}}$ content, vol\% & $50.2 \pm 2.9$ & - & - \\
\hline Porosity, \% & - & $59 \pm 3$ & $35 \pm 4$ \\
\hline${\text { Water flux at } \mathbf{0 . 1 6} \mathbf{~ b a r , ~} \mathbf{m L} / \mathbf{m i n c m}^{2}}^{-}$ & $0.09 \pm 0.02$ & - \\
\hline
\end{tabular}

All measurements $\mathrm{N}=4$, except for pore size $\mathrm{N}=75$.

In previous work, we have prepared porous tubular scaffolds for vascular tissue engineering by sequential dipcoating and salt leaching [25]. Using this technique, it is not possible to fabricate branched structures. A strategy to implement branched vascular structures in scaffolds for 
tissue engineering or organ models is the use of sacrificial templates, e.g. of poly(vinyl alcohol) [16] or carbohydrate [17][39]. The template is immersed in a hydrogel matrix that is crosslinked, after which the template is leached. A draw-back of this approach is the lack of a barrier between e.g. endothelial cells seeded in the channels and other cells present in the surrounding matrix. Therefore, carbohydrate sacrificial templates were coated with thin layers of synthetic polymers such as poly(caprolactone) [17] or poly(lactic-co-glycolic acid) [39]. The layers had a thickness of $10-50 \mu \mathrm{m}$ and were made porous by phase separation or inclusion of leachable $\mathrm{NaCl}$ particles, respectively. Although interesting, the polymer coatings had relatively low tensile strengths around $85 \mathrm{kPa}$ [17] and large pore sizes up to $50 \mu \mathrm{m}$ [39].

Micro-porous vascular structures built by SLA have not been described in literature before. Composites of commercial resins and leachable $\mathrm{NaCl}$ particles were used to print cubes, pyramids and macro-porous scaffolds [40], but not branched vascular structures. Moreover, the smallest particle sizes ranged from $75-180 \mu \mathrm{m}$, resulting in large pores [40]. Printing of branched porous vascular structures by SLA using a cytocompatible polyacrylate has been reported, but the designed pores had a diameter of $100 \mu \mathrm{m}$ which could not be covered by endothelial cells [41]. This problem will not be encountered with the micro-porous vascular structures presented in the present paper.

\section{Conclusions}

Leachable $\mathrm{CaCO}_{3}$ particles with an average size of $0.56 \mu \mathrm{m}$ were found to be suitable as porogen for the preparation of micro-porous PTMC films and vascular structures by casting and SLA, respectively. In order to obtain interconnected pore structures, the $\mathrm{PTMC} / \mathrm{CaCO}_{3}$ composites had to contain at least $30 \mathrm{vol} \% \mathrm{CaCO}_{3}$. The mechanical properties of the microporous PTMC films matched with those of natural blood vessels. The intrinsic permeabilities of the micro-porous PTMC films and vascular structures were at least one order of magnitude higher than reported for the extracellular matrix, indicating no mass transfer limitations in the case of cell seeding.

\section{Acknowledgments:}

The authors acknowledge Huizhou Foryou Medical Device Company for kindly providing TMC monomer. 


\section{References}

[1] H. Bae, A.S. Puranik, R. Gauvin, F. Edalat, B. Carrillo-Conde, N.A. Peppas, A. Khademhosseini, Building vascular networks, Sci. Transl. Med., 4 (2012) 160ps23.

[2] A. Tocchio, M. Tamplenizza, F. Martello, I. Gerges, E. Rossi, S. Argentiere, S. Rodighiero, W. Zhao, P. Milani, C. Lenardi, Versatile fabrication of vascularizable scaffolds for large tissue engineering in bioreactor, Biomaterials, 45 (2015) 124-131.

[3] J.L. West, J.J. Moon, Vascularization of engineered tissues: approaches to promote angiogenesis in biomaterials, Curr. Top. Med. Chem., 8 (2008) 300-310.

[4] M.F. Leong, J.K. Toh, C. Du, K. Narayanan, H.F. Lu, T.C. Lim, A.C. Wan, J.Y. Ying, Patterned prevascularised tissue constructs by assembly of polyelectrolyte hydrogel fibres, Nat. Commun., 4 (2013) 1-8.

[5] S. Kim, H. Lee, M. Chung, NL. Jeon, Engineering of functional, perfusable 3D microvascular networks on a chip, Lab on a Chip, 13 (2013) 1489-1500.

[6] SK. Bhatia, Tissue engineering for clinical applications, Biotechnol. J., 5 (2010) 1309-1323.

[7] T. Dvir, B.P. Timko, D.S. Kohane, R. Langer, Nanotechnological strategies for engineering complex tissues, Nat. Nanotechnol., 6 (2011) 13.

[8] S. Derakhshanfar, R. Mbeleck, K. Xu, X. Zhang, W. Zhong, M. Xing, 3D bioprinting for biomedical devices and tissue engineering: A review of recent trends and advances. Bioact. Mater., 3 (2018) 144-156.

[9] R.K. Jain, P. Au, J. Tam, D.G. Duda, D. Fukumura, Engineering vascularized tissue, Nat. Biotechnol., 23 (2005) 821-823.

[10] E.C. Novosel, C. Kleinhans, P.J. Kluger, Vascularization is the key challenge in tissue engineering, Adv. Drug Deliv. Rev., 63 (2011) 300-311.

[11] FJ. O'brien, Biomaterials \& scaffolds for tissue engineering, Mater. Today, 14 (2011) 8895.

[12] S. Landau, S. Guo, S. Levenberg, Localization of engineered vasculature within 3D tissue constructs, Front. Bioeng. Biotech., 6 (2018) 2. 
[13] C. Vyas, R. Pereira, B. Huang, F. Liu, W. Wang, P. Bartolo, Engineering the vasculature with additive manufacturing, Curr. Opin. Biomed. Eng., 2 (2017) 1-13.

[14] M.D. Sarker, S. Naghieh, N.K. Sharma, X. Chen, 3D biofabrication of vascular networks for tissue regeneration: a report on recent advances, J. Pharm. Anal., 8 (2018) 277-296.

[15] W. Jia, P.S. Gungor-Ozkerim, Y.S. Zhang, K. Yue, K. Zhu, W. Liu, Q. Pi, B. Byambaa, M.R. Dokmeci, S.R. Shin, A. Khademhosseini, Direct 3D bioprinting of perfusable vascular constructs using a blend bioink, Biomaterials, 106 (2016) 58-68.

[16] R. Pimentel, S.K. Ko, C. Caviglia, A. Wolff, J. Emnéus, S.S. Keller, M. Dufva, Threedimensional fabrication of thick and densely populated soft constructs with complex and actively perfused channel network, Acta Biomater., 65 (2018) 174-184.

[17] D. Lei, Y. Yang, Z. Liu, B. Yang, W. Gong, S. Chen, S. Wang, L. Sun, B. Song, H. Xuan, X. Mo, 3D printing of biomimetic vasculature for tissue regeneration, Mater. Horiz., 6 (2019) 1197-1206.

[18] J. Stampfl, S. Baudis, C. Heller, R. Liska, A. Neumeister, R. Kling, A. Ostendorf, M. Spitzbart, Photopolymers with tunable mechanical properties processed by laser-based highresolution stereolithography, J. Micromech. Microeng., 18 (2008) 125014.

[19] S.C. Ligon, R. Liska, J. Stampfl, M. Gurr, R. Mülhaupt, Polymers for 3D printing and customized additive manufacturing, Chem. Rev., 117 (2017) 10212-10290.

[20] G. Chen, T. Ushida, T. Tateishi, Scaffold design for tissue engineering, Macromol. Biosci, 2 (2002) 67-77.

[21] G.J. Zondervan, H.J. Hoppen, A.J. Pennings, W. Fritschy, G. Wolters, R. van Schilfgaard, Design of a polyurethane membrane for the encapsulation of islets of Langerhans, Biomaterials, 13 (1992) 136-144.

[22] O. Guillaume, M.A. Geven, D.W. Grijpma, T.T. Tang, L. Qin, Y.X. Lai, H. Yuan, R.G. Richards, D. Eglin, Poly(trimethylene carbonate) and nano-hydroxyapatite porous scaffolds manufactured by stereolithography, Polym. Adv. Technol., 28 (2017) 1219-1225.

[23] C.J. Pateman, A.J. Harding, A. Glen, C.S. Taylor, C.R. Christmas, PP. Robinson, S. Rimmer, F.M. Boissonade, F. Claeyssens, J.W. Haycock, Nerve guides manufactured from photocurable polymers to aid peripheral nerve repair, Biomaterials, 49 (2015) 77-89. 
[24] L. Elomaa, A. Kokkari, T. Närhi, J.V. Seppälä, Porous 3D modeled scaffolds of bioactive glass and photocrosslinkable poly(e-caprolactone) by stereolithography, Compos. Sci. Technol., 74 (2013) 99-106.

[25] Y. Song, M.M. Kamphuis, Z. Zhang, L.T. Sterk, I. Vermes, A.A. Poot, J. Feijen, D.W. Grijpma, Flexible and elastic porous poly(trimethylene carbonate) structures for use in vascular tissue engineering, Acta Biomater., 6 (2010) 1269-1277.

[26] Z. Guo, D.W. Grijpma, A.A. Poot, Preparation and characterization of flexible and elastic porous tubular PTMC scaffolds for vascular tissue engineering, Polym. Adv. Technol., 28 (2017) 1239-1244.

[27] Z. Zhang, S. Zou, G.J. Vancso, D.W. Grijpma, J. Feijen, Enzymatic surface erosion of poly(trimethylene carbonate) films studied by atomic force microscopy, Biomacromolecules, 6 (2005) 3404-3409.

[28] J.J. Rongen, B. van Bochove, G. Hannink, D.W. Grijpma, P. Buma, Degradation behavior of, and tissue response to photo-crosslinked poly(trimethylene carbonate) networks, J. Biomed. Mater. Res. A, 104 (2016) 2823-2832.

[29] B. van Bochove, D.W. Grijpma, Photo-crosslinked synthetic biodegradable polymer networks for biomedical applications, J. Biomater. Sci. Polym. Ed, 30 (2019) 77-106.

[30] S. Schüller-Ravoo, J. Feijen, D.W. Grijpma, Flexible, elastic and tear-resistant networks prepared by photo-crosslinking poly (trimethylene carbonate) macromers, Acta Biomater., 8 (2012) 3576-3585.

[31] S. Schüller-Ravoo, E. Zant, J. Feijen, D.W. Grijpma, Preparation of a designed poly (trimethylene carbonate) microvascular network by stereolithography, Adv. Healthc. Mater., 3 (2014) 2004-2011.

[32] F. Pennella, G. Cerino, D. Massai, D. Gallo, G.F. Labate, AL. Schiavi, M.A. Deriu, A. Audenino, U. Morbiducci, A survey of methods for the evaluation of tissue engineering scaffold permeability, Annu. Rev. Biomed. Eng, 41 (2013) 2027-2041.

[33] K.Y. Chooi, A. Comerford, S.J. Sherwin, P.D. Weinberg, Intimal and medial contributions to the hydraulic resistance of the arterial wall at different pressures: a combined computational and experimental study, J. R. Soc. Interface, 13 (2016) 20160234. 
[34] B. van Bochove, G. Hannink, P. Buma, D.W. Grijpma, Preparation of designed poly (trimethylene carbonate) meniscus implants by stereolithography: challenges in stereolithography, Macromol. Biosci, 16 (2016) 1853-1863.

[35] S.B. Blanquer, A.W. Gebraad, S. Miettinen, A.A. Poot, D.W. Grijpma, S.P. Haimi, Differentiation of adipose stem cells seeded towards annulus fibrosus cells on a designed poly(trimethylene carbonate) scaffold prepared by stereolithography, J. Tissue Eng. Regen. Med., 11 (2017) 2752-2762.

[36] S.B. Blanquer, S.P. Haimi, A.A. Poot, D.W. Grijpma, Effect of pore characteristics on mechanical properties and annulus fibrosus cell seeding and proliferation in designed PTMC tissue engineering scaffolds, Macromol. Symp., 334 (2013) 75-81.

[37] E. Bat, J.A. Plantinga, M.C. Harmsen, M.J. van Luyn, Z. Zhang, D.W. Grijpma, J. Feijen, Trimethylene carbonate and $\epsilon$-caprolactone based (co) polymer networks: mechanical properties and enzymatic degradation, Biomacromolecules, 9 (2008) 3208-3215.

[38] E. Bat, J.A. Plantinga, M.C. Harmsen, M.J. Van Luyn, J. Feijen, D.W. Grijpma, In vivo behavior of trimethylene carbonate and $\varepsilon$-caprolactone-based (co) polymer networks: Degradation and tissue response, J. Biomed. Mater. Res. A, 95 (2010) 940-949.

[39] Y. Fang, T. Zhang, L. Zhang, W. Gong, W. Sun, Biomimetic design and fabrication of scaffolds integrating oriented micro-pores with branched channel networks for myocardial tissue engineering, Biofabrication, 11 (2019) 035004.

[40] X. Mu, T. Bertron, C. Dunn, H. Qiao, J. Wu, Z. Zhao, C. Saldana, H.J. Q, Porous polymeric materials by 3D printing of photocurable resin, Mater. Horiz., 4 (2017) 442-449.

[41] B. Huber, S. Engelhardt, W. Meyer, H. Krüger, A. Wenz, V. Schönhaar, GE. Tovar, PJ. Kluger, K. Borchers, Blood-vessel mimicking structures by stereolithographic fabrication of small porous tubes using cytocompatible polyacrylate elastomers, biofunctionalization and endothelialization, J. Funct. Biomater., 7 (2016) 11. 


\section{Chapter 8 - Preparation and characterization of flexible and elastic porous tubular PTMC scaffolds for vascular tissue engineering}

Zhengchao Guo, Dirk W. Grijpma, André A. Poot

Department of Biomaterials Science and Technology, University of Twente, Enschede, The Netherlands.

Published

Z. Guo, D.W. Grijpma, A.A. Poot, Preparation and characterization of flexible and elastic porous tubular PTMC scaffolds for vascular tissue engineering, Polym. Adv. Technol., 28 (2017) 1239-1244. 


\begin{abstract}
Vascular grafts with an inner diameter less than $6 \mathrm{~mm}$ are urgently needed due to the increasing prevalence of vascular disease. In this study, tubular scaffolds for vascular tissue engineering were fabricated by photo-crosslinking of acrylate-functionalized poly(trimethylene carbonate) (PTMC) macromers of different molecular weights in a glass mold. Porous structures were prepared by means of salt leaching. Tubular scaffolds were obtained with an inner diameter of $3 \mathrm{~mm}$, a wall thickness of $1 \mathrm{~mm}$ and a length of $4.5 \mathrm{~cm}$. Pore sizes ranged from 0-290 $\mu \mathrm{m}$ and the porosity was around $70 \%$. The pores were homogeneously distributed and interconnected.

PTMC macromers with a molecular weight of 4, 8, 13, 17 and $22 \mathrm{~kg} / \mathrm{mol}$ were used. With increasing PTMC macromer molecular weight from 4 to $22 \mathrm{~kg} / \mathrm{mol}$, the E-modulus and maximum tensile strength of the scaffolds in the radial direction increased from 0.56 to 1.12 $\mathrm{MPa}$ and 0.12 to $0.55 \mathrm{MPa}$, respectively. Stress-strain curves for scaffolds made of 13,17 and $22 \mathrm{~kg} / \mathrm{mol}$ PTMC macromers, showed a 'toe' region characteristic for native arteries, followed by a linear increase until the maximum stress was reached.

The E-moduli of the latter scaffolds are comparable to those of native arteries, whereas the maximum tensile strengths are approximately 4-fold lower. This can be improved, however, by cell seeding in the scaffolds and subsequent mechanical stimulation in a bioreactor. It is concluded that the porous tubular scaffolds made of 13, 17 and $22 \mathrm{~kg} / \mathrm{mol}$ PTMC macromers are suitable scaffolds for vascular tissue engineering.
\end{abstract}

\title{
1 Introduction
}

Peripheral vascular disease is in most cases related to narrowing or occlusion of arteries and affects 12 to $20 \%$ of the population older than 65 years [1]. Peripheral vascular disease can be treated by placement of a bypass graft, for which purpose autologous blood vessels, allografts, xenografts, or synthetic grafts of Dacron or Teflon are being used. These have all limitations such as low availability, immune responses, and low patency rates, [2] which may be overcome by vascular tissue engineering.

The purpose of vascular tissue engineering is to produce tubular grafts with similar properties as the native blood vessels that should be replaced.3 Current strategies to fabricate such grafts include scaffold-guided tissue engineering and scaffold - free approaches such as assembly by 
cell sheet technology and 3D bioprinting [4-6] In view of the required mechanical properties of the grafts, design considerations as well as relatively low costs, a large amount of research has focused on scaffold - guided tissue engineering [7]. In addition to the use of biocompatible materials and reproducible fabrication methods, the scaffold must have an appropriate structure for the intended application, be sufficiently strong to bear forces in vivo, and promote cell attachment and growth of new tissue. To fulfil the latter requirement, the scaffold should consist of an interconnected porous structure [8].

Porous tubular scaffolds can be prepared by combined solvent casting on a rod and particulate leaching, electrospinning on a rotating rod, and 3D printing of designed structures [9-11] Although solvent casting of a polymer solution containing leachable particles is feasible, 9 this method has a relatively low consistency in terms of wall thickness of the scaffold. Although electrospinning is a popular technique for the fabrication of tissue engineering scaffolds, these may suffer from poor cell penetration due to small pore sizes and poor control over mechanical properties [10]. 3D printing is a promising technique to be applied in vascular tissue engineering. Not all polymers, however, are appropriate for 3D printing, and scaffolds may have unsuitable mechanical properties [12]. Thus, the development of a new method to fabricate suitable porous tubular scaffolds for vascular tissue engineering is imperative.

In view of its biocompatibility, biodegradability, and rubber-like mechanical properties, poly(trimethylene carbonate) (PTMC) is an excellent polymer for tissue engineering applications. Acrylate-functionalized PTMC macromers can easily be processed and photocrosslinked for the preparation of scaffolds [13]. Salt leaching has widely been used to create porous structures [14]. In this study, we investigated the preparation of porous tubular scaffolds for vascular tissue engineering by means of photo-crosslinking of acrylate-functionalized PTMC macromers in a glass mold combined with salt leaching.

\section{Materials and Methods}

\subsection{Materials and equipment}

Trimethylene carbonate (1,3-dioxane-2-one, TMC) was provided by Huizhou Foryou Medical Devices (Shenzhen, China). 1,1,1 - Tris(hydroxymethyl)propane (trimethylolpropane) was purchased from Fluka. Tin (II) ethylhexanoate [Sn(Oct)2], hydroquinone, methacrylic anhydride (MA), trimethylamine, and Irgacure 2959 were obtained from Sigma - Aldrich. 
Propylene carbonate (PC) and calcium hydride were ordered from Merck Millipore. Dichloromethane and ethanol (99.8\%) were bought from VWR Chemicals. Dichloromethane was dried by calcium hydride and distilled under argon atmosphere. Salt particles were sieved to a size of 250 to $425 \mu \mathrm{m}$ by using stainless steel sieves and subsequently dried in an oven at $120^{\circ} \mathrm{C}$ for 2 days. The glass mold consisted of two quartz glass tubes with different diameters, which were custom ordered from Technical Glass Products (Painesville, USA). MilliQ water was prepared by a Millipore Advantage A10 from Merck Millipore.

\subsection{PTMC-MA synthesis and formulation of PTMC-MA/PC/salt resin}

Three-armed PTMC was synthesized by ring-opening polymerization of TMC, using $\mathrm{Sn}(\mathrm{Oct})_{2}$ as catalyst and trimethylolpropane as initiator, under argon at $130^{\circ} \mathrm{C}$ for 2 days. PTMC molecular weight was controlled by varying the amount of initiator. Three-armed PTMC was subsequently converted to PTMC - MA macromer as described by Geven et al [13]. Threearmed PTMC oligomer and PTMC-MA macromer were analyzed by ${ }^{1} \mathrm{H}-\mathrm{NMR}$ ((Bruker Ascend 400/Avance III $400 \mathrm{MHz}$ NMR spectrometer) to determine the average molecular weight (Mn), conversion of TMC, and degree of functionalization with MA.

Photo-crosslinkable resin was prepared by dissolving PTMC-MA macromer in PC for 2 days, after which Irgacure 2959 (2.5 wt\% relative to PTMC-MA) was added as photo-initiator under stirring for $1 \mathrm{hr}$. Subsequently, dried salt particles were added to the solution that was mixed by hand with a spoon. The weight ratio of PTMC-MA: PC: salt was 1:4:9.

\subsection{Photo-crosslinking of PTMC-MA/PC/salt resin and post-processing}

Annular glass molds with an inner and outer diameter of 5 and $8 \mathrm{~mm}$, respectively, were filled with PTMC-MA/PC/salt resins containing 4, 8, 13, 17, or $22 \mathrm{~kg} / \mathrm{mol}$ PTMC-MA. Pieces of plastic tube with an inner diameter of $5 \mathrm{~mm}$ and a wall thickness of $1.5 \mathrm{~mm}$ were used as stoppers. UV crosslinking was carried out for $30 \mathrm{~min}$ at $365 \mathrm{~nm}$ in an UltraLum crosslinking cabinet. In order to obtain homogeneous crosslinking and to keep a homogeneous distribution of the salt particles, the molds were turned 6 times for $180^{\circ}$.

After cooling to room temperature, the inner tube of the mold could be easily removed. To remove the outer tube of the mold, the photo-crosslinked structure was soaked for 2 days in a 
PC/ethanol solution $(3: 7, \mathrm{v} / \mathrm{v})$. Subsequently, the PC was removed by extraction of the structures in a graded series of PC/ethanol solutions, starting at a ratio of 8:2 until 0:10 (v/v). The extraction solutions were refreshed daily. After extraction, the solid structures containing ethanol were dried at $50^{\circ} \mathrm{C}$ for $12 \mathrm{hr}$. Finally, the salt particles were leached from the tubes by soaking for 2 days in MilliQ water. After rinsing with ethanol, the porous tubular crosslinked PTMC scaffolds were dried at $30^{\circ} \mathrm{C}$ for 2 days under vacuum. A schematic representation of the fabrication method is shown in Figure. 1.

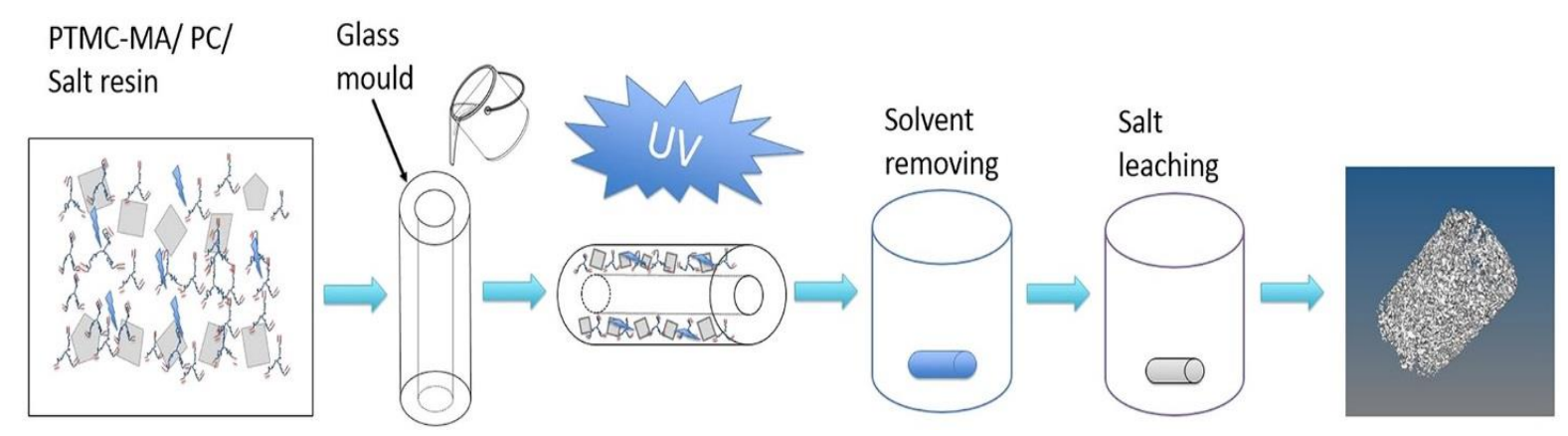

Figure 1. Schematic representation of the preparation of porous tubular crosslinked PTMC scaffolds.

\subsection{Characterization of porous tubular PTMC scaffolds}

The porosity of the tubular crosslinked PTMC scaffolds was determined gravimetrically according to Equation 1,

Porosity $=\left[1-\left(\mathrm{W}_{\mathrm{dry}} / \mathrm{V}_{\mathrm{dry}} * \rho\right.\right.$ PTMC $\left.)\right] * 100 \%$

in which $\rho_{\text {PTMC }}=1.31 \mathrm{~g} / \mathrm{cm}^{3}, \mathrm{~W}_{\text {dry }}$ is the dry weight of the scaffold, and $\mathrm{V}_{\text {dry }}$ is the volume of the scaffold in dry condition (regarded as a solid tube).

The pore size distribution of the tubular crosslinked PTMC scaffolds was determined by scanning electron microscopy (SEM), using a Hitachi S800 operating at $5 \mathrm{kV}$. Prior to imaging, dry specimens were coated with gold-platinum by using a Polaron E5600 sputter coater.

Micro-computed tomography (Micro-CT) was used to obtain a three-dimensional visualization of the pore structures of the scaffolds. A General Electric Explore Locus SP was used operating 
at $90 \mathrm{kV}$ and $80 \mu \mathrm{A}$. The resolution was $14 \mu \mathrm{m}$. Average pore size and porosity were calculated from the data generated with scaffolds in dry condition.

The tensile properties of dry porous tubular crosslinked PTMC scaffolds were determined in the radial direction according to standards of the American National Standards Institute and the Association for the Advancement of Medical Instrumentation (ANSI/AAMI VP20: 1994) at a pulling rate of $1 \mathrm{~mm} / \mathrm{min}$, using a Zwick Z020 tensile tester. The initial stiffness was determined from the slope of the tensile curve between 5 and $15 \%$ of strain.

\section{Results and Discussion}

\subsection{Characterization of PTMC-MA}

The average molecular weight $(\mathrm{Mn})$ of the three-armed PTMC oligomers, conversion of TMC monomer, and degree of functionalization of the oligomers with MA are shown in Table 1. All monomer conversions were higher than 99\%, and the molecular weights of the PTMC oligomers were close to the intended values. The degree of functionalization of the oligomers with MA ranged from 87 to $96 \%$, indicating that all PTMC macromers contained enough double bonds for efficient crosslinking.

Table 1. Characterization of the three-armed PTMC-MA macromers

\begin{tabular}{lccccc}
\hline & $\mathbf{4 k}$ & $\mathbf{8 k}$ & $\mathbf{1 3 k}$ & $\mathbf{1 7 k}$ & $\mathbf{2 2 k}$ \\
\hline $\begin{array}{l}\text { PTMC macromer } \\
\text { molecular weight } \\
\text { (Mn), g/mol }\end{array}$ & 4460 & 7980 & 13600 & 16980 & 22300 \\
\hline $\begin{array}{l}\text { Monomer conversion } \\
\text { Degree of } \\
\text { functionalization }\end{array}$ & $99.8 \%$ & $99.8 \%$ & $99.6 \%$ & $99.3 \%$ & $99.4 \%$ \\
\hline
\end{tabular}

Intended molecular weights are abbreviated as $4,8,13,17$, and $22 \mathrm{k}$. 


\subsection{Characterization of porous tubular crosslinked PTMC scaffolds}

3.2.1 Physical parameters of the scaffolds

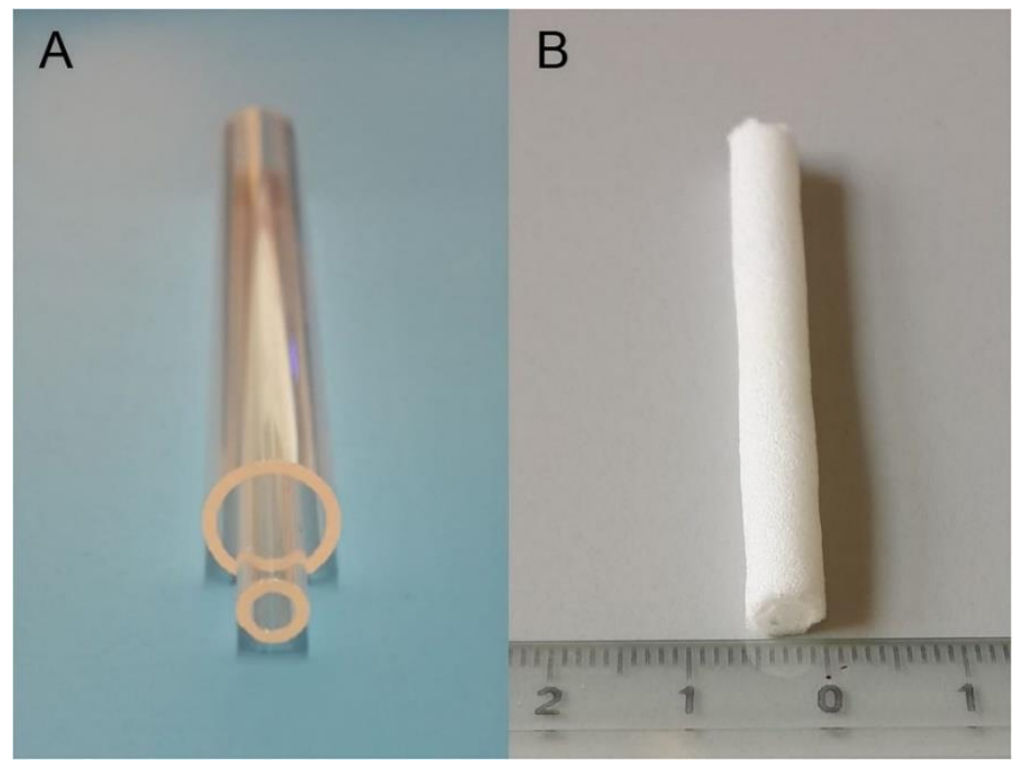

Figure 2. Glass mold (A) and macroscopic appearance of a porous tubular crosslinked PTMC scaffold after salt leaching $(B)$.

Figure 2 shows the glass mold used for the preparation of the crosslinked PTMC scaffolds as well as a macroscopic picture of one of the tubes after de-molding and post-processing. The annular space of the mold had an inner and outer diameter of 5 and $8 \mathrm{~mm}$, respectively. The inner and outer diameters of the tubular PTMC scaffolds, however, were smaller. As shown in Table 2, the scaffolds had an inner diameter around $3 \mathrm{~mm}$ and a wall thickness of approximately $1 \mathrm{~mm}$ (i.e. an outer diameter of $5 \mathrm{~mm}$ ). This difference can be explained by shrinkage of the scaffolds during post-processing. Photo-crosslinking of the PTMC-MA macromer took place in the swollen state, due to the presence of PC, resulting in contraction of the PTMC network upon extraction of PC and subsequent salt leaching. Also, the length of the scaffolds decreased, from approximately $7 \mathrm{~cm}$ after photo-crosslinking in the mold to $4.5 \mathrm{~cm}$ after post-processing (data not shown).

As shown in Table 2, the porosities of the scaffolds as determined by gravimetry amounted to approximately $75 \%$. The theoretical porosity based on the volume ratio of salt to PTMC was 83\%. This discrepancy can also be explained by shrinkage of the structures during extraction 
and salt leaching. Porosities determined by Micro-CT were around 67\% (Table 2). This is lower than the gravimetrical data, probably because of the Micro-CT resolution of $14 \mu \mathrm{m}$.

Table 2. Physical parameters of porous tubular crosslinked PTMC scaffolds

\begin{tabular}{|c|c|c|c|c|c|c|}
\hline Parameters & $4 k$ & $8 \mathbf{k}$ & $13 k$ & $17 \mathbf{k}$ & $22 k$ & $\mathbf{B 5 0}^{\mathrm{a}}$ \\
\hline $\begin{array}{l}\text { Inner diameter } \\
\text { b }, \mathbf{m m}\end{array}$ & $2.96 \pm 0.06$ & $3.00 \pm 0.06$ & $3.12 \pm 0.09$ & $3.16 \pm 0.8$ & $3.18 \pm 0.8$ & $3.15 \pm 0.28$ \\
\hline $\begin{array}{l}\text { Wall thickness }{ }^{\mathbf{b}} \text {, } \\
\text { mm }\end{array}$ & $0.95 \pm 0.04$ & $0.98 \pm 0.04$ & $1.04 \pm 0.04$ & $1.05 \pm 0.04$ & $1.07 \pm 0.04$ & $0.83 \pm 0.25$ \\
\hline Porosity $^{\mathrm{c}}$, vol \% & $73 \pm 2.1$ & $73 \pm 2.6$ & $75 \pm 4.3$ & $76 \pm 3.3$ & $77 \pm 3.1$ & $82.2 \pm 2.0$ \\
\hline Porosity $^{\mathrm{d}}$, vol \% & $61 \pm 3.2$ & $64 \pm 2.3$ & $67 \pm 2.1$ & $69 \pm 1.2$ & $68 \pm 2.6$ & $77.8 \pm 1.7$ \\
\hline $\begin{array}{l}\text { Average pore } \\
\text { size }^{d}, \mu \mathrm{m}\end{array}$ & 81 & 81 & 87 & 91 & 93 & 108 \\
\hline $\begin{array}{l}\text { Pore size range } \\
\text { e }, \mu \mathrm{m}\end{array}$ & $0-280$ & $0-283$ & $0-286$ & $0-294$ & $0-289$ & $0-308$ \\
\hline
\end{tabular}

a Porous tubular PTMC scaffold prepared by Song et al. by dip coating, crosslinking by $\gamma$-irradiation, followed by salt leaching. [9]

b Inner diameter and wall thickness of all porous tubular PTMC scaffolds were measured by using a caliper.

c Porosity as determined by gravimetry.

d Porosity and average pore size as determined by Micro-CT.

e Pore size range as determined by SEM.
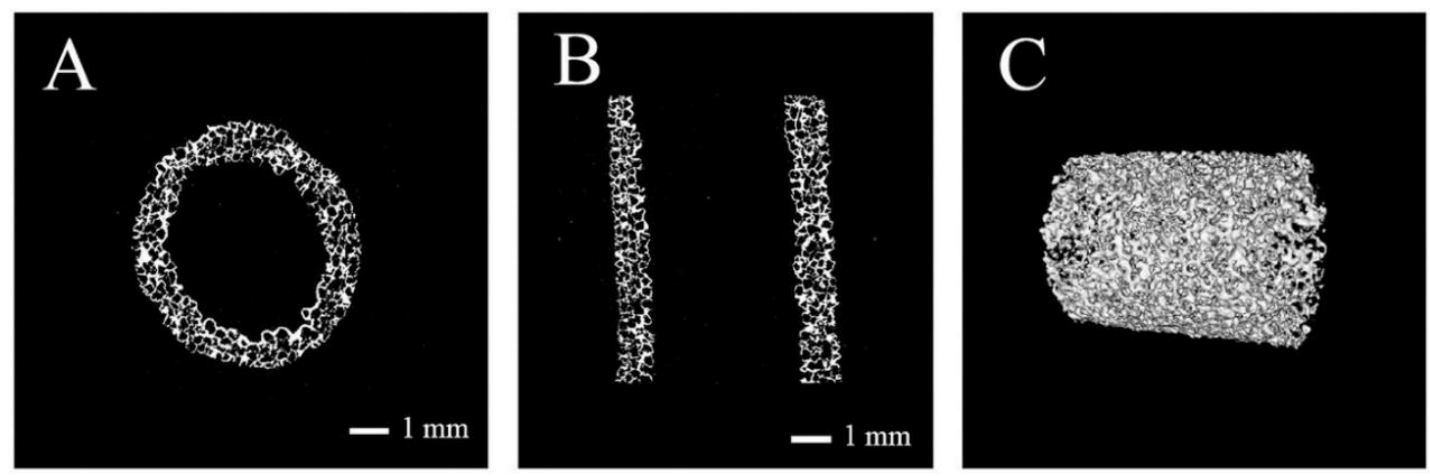

Figure 3. Micro-CT images of a porous tubular crosslinked PTMC scaffold. Transversal cross-section (A), longitudinal cross-sections (B), and generated $3 D$ image $(C)$. 


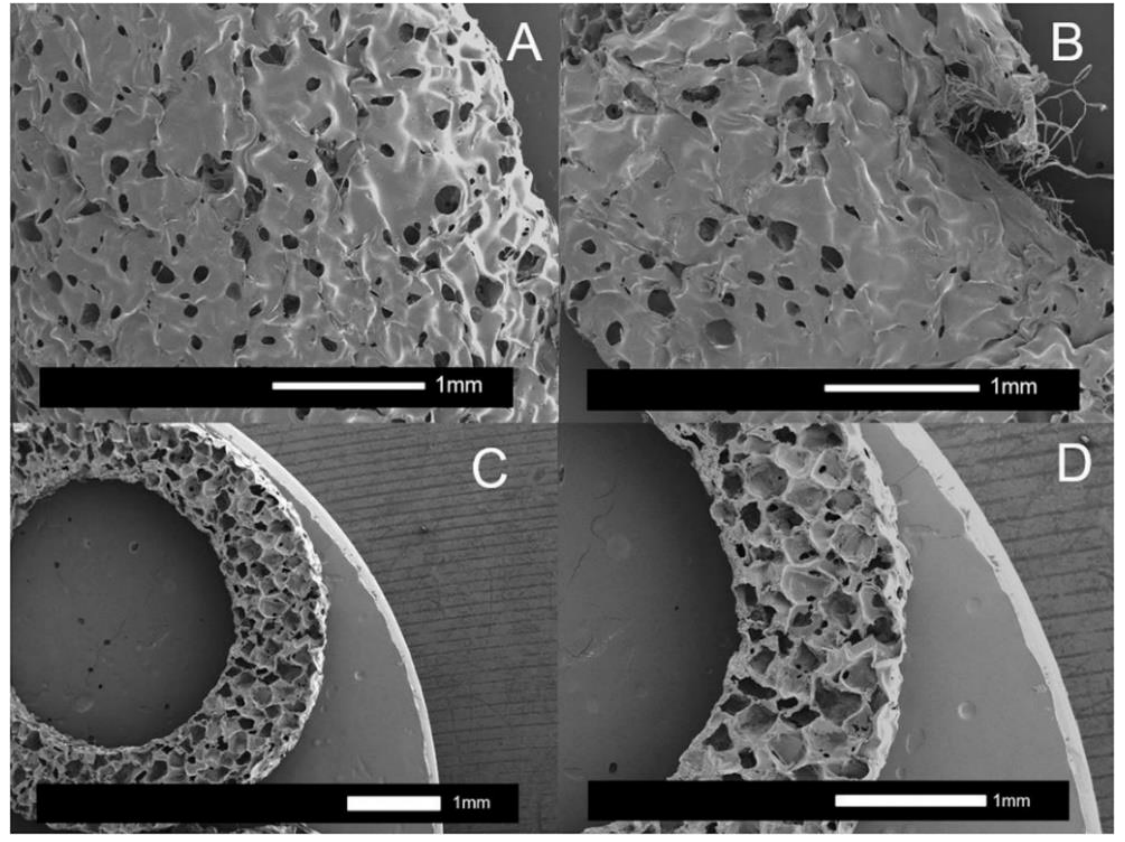

Figure 4. SEM images of a porous tubular crosslinked PTMC scaffold. Outer surface (A), inner surface $(B)$, and cross sections $(C)$ and $(D)$.

The average pore size of the scaffolds as determined by Micro-CT was approximately $87 \mu \mathrm{m}$. Micro-CT imaging showed that the pores were homogeneously distributed throughout the scaffolds (Figure. 3). Pore sizes determined by SEM ranged from 0 to $290 \mu \mathrm{m}$ (Table 2). This is smaller than the sizes of the salt particles $(250-425 \mu \mathrm{m})$, again due to shrinkage of the structures during post-processing. SEM imaging showed that both the outer and inner surfaces of the wall of the scaffolds had an open porous structure (Figure. 4). Moreover, cross-sections of the scaffolds showed that the pores were interconnected. As reported by Yannas, human aortic smooth muscle cells in suspension have a length and diameter of approximately 55 and $8 \mu \mathrm{m}$, respectively, and the optimal pore size for migration of the cells through a scaffold is around $100 \mu \mathrm{m}$ [15]. Thus, the pore characteristics of the scaffolds meet the requirements for successful cell seeding and migration.

Table 2 also shows that the dimensions, porosities, and pore sizes of the crosslinked 4 and $8 \mathrm{k}$ PTMC scaffolds were lower than those of the scaffolds prepared with the higher molecular weight PTMC-MA macromers. The reason for this is that the crosslinked 4 and $8 \mathrm{k}$ PTMC scaffolds shrunk more during extraction because of the shorter chain lengths between crosslinks and thus a higher crosslink density. Finally, Table 2 shows comparable physical parameters of porous tubular PTMC scaffolds (B50) fabricated by Song et al., by casting of 
high molecular weight linear PTMC/salt suspension on a glass rod, followed by crosslinking by means of $\gamma$-irradiation and leaching of the salt particles in water [9]. As those PTMC scaffolds proved to be very suitable for vascular tissue engineering, this can also be expected for the scaffolds prepared in the present study.

\subsubsection{Mechanical properties of the scaffolds}

For application in vascular tissue engineering, the mechanical properties of the scaffolds are very important. After seeding of cells, e.g. smooth muscle cells or stem cells, in the pores of the scaffolds, subsequent mechanical stimulation in a bioreactor is most effective when the mechanical properties of the constructs comply with those of native arteries. This especially holds for the mechanical properties in radial direction. The pulsatile flow of blood in vivo causes cyclic distension of the arterial wall, which in turn affects cell differentiation, function, and orientation.

As shown in Table 3, the initial stiffness (E-modulus) and maximum tensile strength in the radial direction of the porous tubular crosslinked scaffolds increased from 0.56 to $1.12 \mathrm{MPa}$ and 0.12 to $0.55 \mathrm{MPa}$, respectively, with increasing PTMC-MA macromer molecular weight from 4 to $22 \mathrm{~kg} / \mathrm{mol}$. The increasing tensile strength with increasing macromer molecular weight is in agreement with data reported by Schüller-Ravoo et al. for photo - crosslinked PTMC network films [16]. In the latter study, however, the E-modulus decreased with increasing PTMC-MA macromer molecular weight from 3 to $24 \mathrm{~kg} / \mathrm{mol}$. Using $3 \mathrm{k}$ macromer, densely crosslinked brittle films were obtained, whereas 18, 20, and $24 \mathrm{k}$ macromers yielded rubber - like elastomeric films. It should be noted that the scaffolds in the present study were porous. Increasing porosity decreases the mechanical properties of scaffolds, especially the Emodulus. As the porosities of the tubular crosslinked PTMC scaffolds were the same, this cannot explain the lower E-moduli of the scaffolds prepared with the lower molecular weight macromers, most likely, this was caused by the processing conditions that were used. The tubular PTMC scaffolds were crosslinked in the presence of the non-reactive diluent PC and salt particles. Upon extraction of the PC, the brittle networks formed with the lower molecular weight macromers may have been partially damaged because of contraction while the salt particles were still in place. Subsequent salt leaching resulted in porous scaffolds with lower E-moduli. 
Table 3. Tensile properties of porous tubular crosslinked PTMC scaffolds and natural arteries in the radial direction

\begin{tabular}{|c|c|c|c|}
\hline Sample & $\begin{array}{c}\text { Initial stiffness, } \\
\text { MPa }\end{array}$ & $\begin{array}{l}\text { Maximum tensile } \\
\text { strength, MPa }\end{array}$ & Elongation, \% \\
\hline $4 k$ & $0.56 \pm 0.09$ & $0.12 \pm 0.05$ & $382 \pm 40$ \\
\hline $8 \mathbf{k}$ & $0.53 \pm 0.06$ & $0.25 \pm 0.03$ & $820 \pm 60$ \\
\hline 13k & $0.79 \pm 0.09$ & $0.32 \pm 0.03$ & $1130 \pm 150$ \\
\hline $17 k$ & $0.89 \pm 0.12$ & $0.41 \pm 0.04$ & $1320 \pm 180$ \\
\hline $22 k$ & $1.12 \pm 0.30$ & $0.55 \pm 0.03$ & $1080 \pm 160$ \\
\hline B-50 ${ }^{\text {a }}$ & $1.10 \pm 0.08$ & $0.17 \pm 0.04$ & $1214 \pm 120$ \\
\hline Ovine Carotid artery ${ }^{a}$ & $1.21 \pm 0.14$ & $1.65 \pm 0.08$ & $255 \pm 21$ \\
\hline $\begin{array}{l}\text { Human arteria } \\
\text { mesenterica inferior }{ }^{\text {a }}\end{array}$ & 5.7 & 1.89 & 345 \\
\hline
\end{tabular}

a. Data from Song et al. [4]. B-50 refers to a porous tubular PTMC scaffold prepared by dip coating, crosslinking by $\gamma$-irradiation, followed by salt leaching.

The number of acrylate groups available for photo-crosslinking decreased with increasing PTMC-MA macromer molecular weight. Because of this and the relatively large amount of non-reactive diluent present in the resins, it was expected that the efficiency of photocrosslinking would decrease with increasing PTMC-MA macromer molecular weight. Indeed, crosslinked scaffolds could not be formed using macromers with a molecular weight higher than $22 \mathrm{~kg} / \mathrm{mol}$.

Whereas the E-modulus of the 13, 17, and $22 \mathrm{k}$ scaffolds was comparable to that of the B50 scaffold previously prepared by Song et al. [9], the maximum tensile strength of the scaffolds prepared in the present study was higher. Stress-strain curves for the 13, 17, and $22 \mathrm{k}$ scaffolds showed a "toe" region characteristic for native arteries, followed by a linear increase until the maximum stress was reached 17 (Figure. 5). Elongations at break were higher than $1000 \%$. The E-moduli of the 13,17 , and $22 \mathrm{k}$ scaffolds are comparable to those of native arteries, whereas the maximum tensile strengths are approximately fourfold lower (Table 3 ). This can be improved, however, by cell seeding in the scaffolds and subsequent mechanical stimulation as described earlier. 


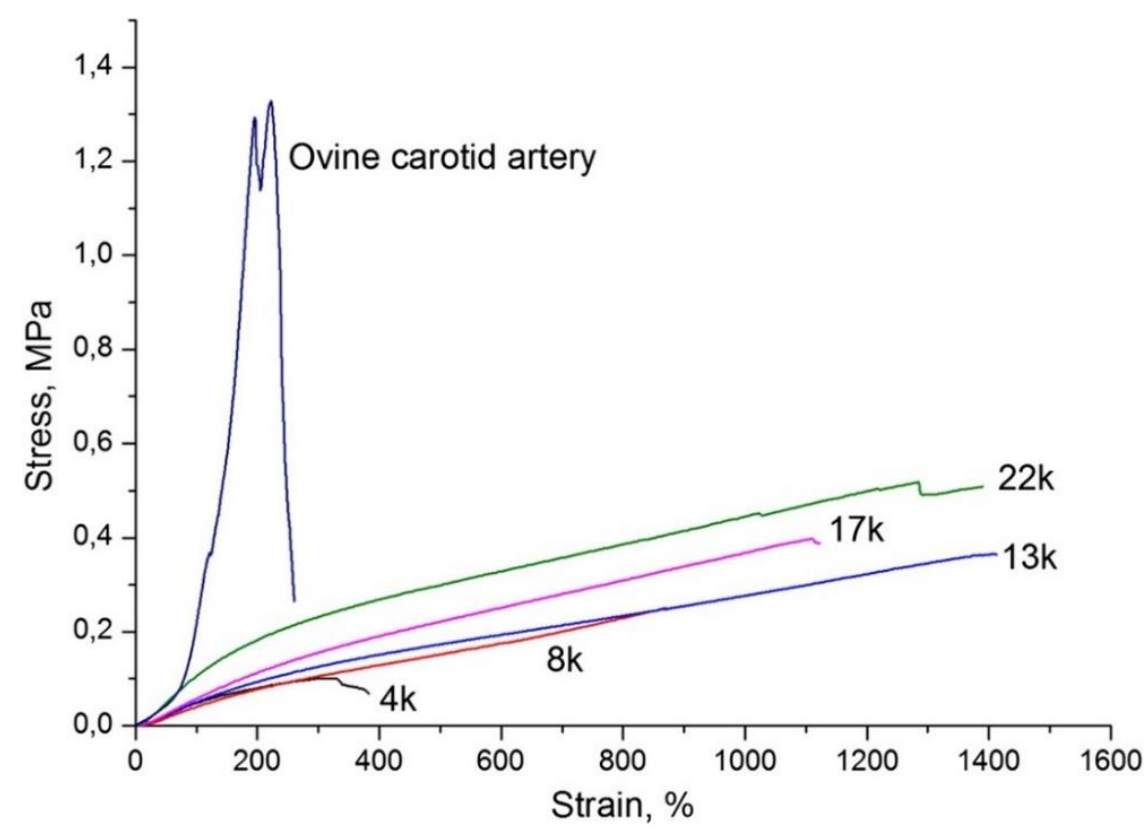

Figure 5. Stress-strain measurements of the porous tubular crosslinked PTMC scaffolds and an ovine carotid artery.

\section{Conclusions}

This study shows that photo-crosslinking of acrylate-functionalized PTMC macromers in a glass mold followed by salt leaching is a feasible method to prepare suitable scaffolds for vascular tissue engineering. Although the tubular crosslinked PTMC scaffolds shrink during post-processing, this can be taken into account during fabrication. Desired scaffold dimensions can be obtained by adjusting the dimensions of the mold. In view of their mechanical properties, porous tubular scaffolds made of PTMC macromers with a molecular weight of $13-22 \mathrm{~kg} / \mathrm{mol}$ are the preferred scaffolds for vascular tissue engineering.

\section{Acknowledgements}

The authors would like to thank the China Scholarship Council for financial support. 


\section{References}

[1] http://www.healthline.com/health/peripheral-vascular-disease

[2] P. Zilla, D. Bezuidenhout, P. Human, Prosthetic vascular grafts: wrong models, wrong questions and no healing, Biomaterials, 28 (2007) 5009-5027.

[3] D. G. Seifu, A. Purnama, K. Mequanint, D. Mantovani, Small-diameter vascular tissue engineering, Nat. Rev. Cardiol., 10 (2013) 410.

[4] A. Patel, B. Fine, M. Sandig, K. Mequanint, Elastin biosynthesis: The missing link in tissueengineered blood vessels, Cardiovasc. Res., 71 (2006) 40-49.

[5] N. L'Heureux, S. Paquet, R. Labbe, L. Germain, F.A. Auger, A completely biological tissueengineered human blood vessel, FASEB J, 12 (1998) 47-56.

[6] C. Norotte, F.S. Marga, L.E. Niklason, G. Forgacs, Scaffold-free vascular tissue engineering using bioprinting, Biomaterials, 30 (2009) 5910-5917.

[7] J.D. Berglund, Z.S. Galis, Designer blood vessels and therapeutic revascularization, Br. J. Pharmacol., 140 (2003) 627-636.

[8] I. Martin, D. Wendt, M. Heberer, The role of bioreactors in tissue engineering, Trends Biotechnol, 22 (2004): 80-86.

[9] Y. Song, M. M. J. Kamphuis, Z. Zhang, L. M. T. Sterk, I. Vermes, A.A. Poot, J. Feijen, D.W. Grijpma, Flexible and elastic porous poly (trimethylene carbonate) structures for use in vascular tissue engineering, Acta biomater., 6 (2010) 1269-1277.

[10] A. Hasan, A. Memic, N. Annabi, M. Hossain, A. Paul, M.R. Dokmeci, F. Dehghani, A. Khademhosseini, Electrospun scaffolds for tissue engineering of vascular grafts, Acta biomater., 10 (2014) 11-25.

[11] M.A. Cleary, E. Geiger, C. Grady, C. Best, Y. Naito, C. Breuer, Vascular tissue engineering: the next generation, Trends Mol. Med., 18 (2012) 394-404.

[12] S. Baudis, F. Nehl, S.C. Ligon, A. Nigisch, H. Bergmeister, D. Bernhard, J. Stampfl, R. Liska, Elastomeric degradable biomaterials by photopolymerization-based CAD-CAM for vascular tissue engineering, Biomed. Mater., 6 (2011) 055003. 
[13] M.A. Geven, D. Barbieri, H. Yuan, J.D. de Bruyn, D.W. Grijpma, Preparation and mechanical properties of photo-crosslinked poly (trimethylene carbonate) and nanohydroxyapatite composites, Clin. Hemorheol. Microcirc., 60 (2015) 3-11.

[14] D.W. Hutmacher, Scaffold design and fabrication technologies for engineering tissues: state of the art and future perspectives, J. Biomater. Sci. Polym. Ed., 12 (2001) 107-124.

[15] I..V. Yannas, Tissue regeneration templates based on collagen-glycosaminoglycan copolymers, Biopolymers II. Springer, Berlin, Heidelberg, (1995) 219-244.

[16] S. Schüller-Ravoo, J. Feijen, D.W. Grijpma, Flexible, elastic and tear-resistant networks prepared by photo-crosslinking poly (trimethylene carbonate) macromers, Acta biomater., 8 (2012) 3576-3585.

[17] Y.C. Fung, Mechanical properties and active remodeling of blood vessels. In: Biomechanics. Mechanical Properties of Living Tissues. Springer, New York, (1993) 321-391. 


\section{Appendix - Dynamic culturing of smooth muscle cells in porous tubular poly(trimethylene carbonate) scaffolds}

Zhengchao Guo, Julia Teders, Dirk Grijpma, André Poot.

Department of Biomaterials Science and Technology, Faculty of Science and Technology, University of Twente, Enschede, The Netherlands. 


\begin{abstract}
Tissue-engineered vascular grafts for the replacement of diseased blood vessels are expected to be clinically applied in the coming years. To engineer such grafts, patient-specific vascular cells have to be seeded and matured in porous tubular scaffolds. In this study, a porous tubular scaffold was prepared by photo-crosslinking of a resin of three-armed end-methacrylated poly(trimethylene carbonate) (PTMC-MA) and $\mathrm{NaCl}$ particles, followed by photo-crosslinking and particle leaching. The tubular scaffolds, with $3 \mathrm{~mm}$ inner diameter, $1 \mathrm{~mm}$ wall thickness and an average pore size of $68 \mu \mathrm{m}$, were seeded with human smooth muscle cells (SMCs) and cultured under dynamic conditions in a pulsatile flow bioreactor. Culturing of SMCs in porous PTMC scaffolds under static conditions was used for comparison. Cells were cultured for 14 days, after which cell proliferation, histology and mechanical properties were evaluated. A three times higher number of SMCs was found using dynamic culture conditions compared to static culturing. Histological analysis of the constructs cultured under dynamic conditions showed that SMCs fully filled the porous scaffold, which was not found after static culturing. An increase in maximum stress of the constructs was found after dynamic culturing compared to static conditions. After endothelialization of the dynamically cultured constructs, future in vivo implantation studies may show the potential of these vascular grafts for clinical application.
\end{abstract}

\title{
1 Introduction
}

Small-diameter vascular grafts have a potential application in replacing diseased or occluded blood vessels, which affects 12 to $20 \%$ of persons older than 65 years [1][2]. Vascular tissue engineering is regarded as a promising method to prepare such vascular grafts. A tissueengineered implant may show significant improvement in immune response and patency compared to current Dacron or Teflon vascular grafts [3]. However, up to now no commercial product has come to the market.

Tissue engineering of small-diameter blood vessels consists of four main steps; isolating patient-specific cells, cell seeding in porous scaffolds, maturation of the construct in a bioreactor and implantation of the final construct in the patient [4][5]. A porous scaffold is used as a temporary support for smooth muscle cells (SMCs) and endothelial cells. A bioreactor provides mechanical mimicking of the cellular environment and accelerates tissue formation. 
A key issue in current vascular tissue engineering remains the manufacturing of a suitable porous tubular scaffold.

Both scaffold materials and manufacturing processes are important in the preparation of vascular grafts. The synthetic polymer poly(trimethylene carbonate) (PTMC) has been used in many soft tissue engineering applications due to its biodegradability and mechanical properties [6][7]. Compared to natural polymers like gelatin, chitosan and silk, PTMC has a low swelling which makes this material highly suitable for use in vivo [1][4]. Compared to other degradable synthetic polymers such as poly(ester urethane)s and polyesters like poly( $\varepsilon$-caprolactone) and poly(lactide)s, an adjustable degradation time, surface erosion mechanism and no formation of acidic degradation products are characteristic for PTMC [8][9]. Several manufacturing techniques including solvent casting, electrospinning and printing have been used to fabricate 3D scaffolds [10][11][12]. Although electrospun vascular scaffolds may have good cell attachment, their small pore size below $20 \mu \mathrm{m}$ does not provide enough space for the growth of SMCs. In addition, the mechanical properties of constructs are not easy to control using this method [13][14]. 3D printing of vascular grafts recently received a lot of attention due to its efficiency and versatility in design. However, current 3D printing technologies do not allow the fabrication of highly porous scaffolds due to limitations of 3D printing devices and materials [15].

In this respect, solvent casting of polymer/leachable particle mixtures still is a feasible option to adjust both pore size and mechanical properties of scaffolds. In a previous study, high molecular weight PTMC combined with sodium chloride or sucrose particles were used to prepare tubular scaffolds by dip-coating on a glass mandrel. Crosslinking by gamma irradiation afforded compliant and creep-resistant PTMC networks, and porous scaffolds were obtained by means of particle leaching in water [16][17][18] [19]. Seeded SMCs were able to effectively attach and grow in these scaffolds having pore sizes ranging from 55-116 $\mu \mathrm{m}$. Although solvent casting of a polymer solution containing leachable particles is feasible, this method has a low consistency in terms of wall thickness of the scaffold.

In this study, we improved the manufacturing method and changed the materials based on the work of Song et al. [17]. Photo-crosslinking of end-methacrylated low molecular weight PTMC has proven to provide structures with similar mechanical and biological properties as the gamma-irradiated scaffolds [20]. We prepared porous tubular scaffolds by photocrosslinking of a mixture of three-armed end-methacrylated PTMC (PTMC-MA) and NaCl 
particles in a mold followed by particle leaching [21]. The obtained porous scaffolds were seeded with SMCs. A pulsatile flow bioreactor was used to mimic the native cell environment and to study cell proliferation and changes in mechanical properties of the constructs.

\section{Materials and Methods}

\subsection{Materials}

Trimethylene carbonate (TMC) was obtained from Huizhou Foryou Medical Devices. 1,1,1Tris(hydroxymethyl)propane (trimethylolpropane) was purchased from Fluka. Tin (II)ethylhexanoate $\left(\mathrm{Sn}(\mathrm{Oct})_{2}\right)$, hydroquinone, methacrylic anhydride (MA), triethylamine (TEA) and Irgacure 2959 were purchased from Sigma-Aldrich. Propylene carbonate (PC) was ordered from Merck Millipore. Dichloromethane and ethanol were bought from VWR Chemicals. Sodium chloride particles were sieved to a size of $106-250 \mu \mathrm{m}$ and dried at $120{ }^{\circ} \mathrm{C}$ for 2 days before use. Quartz glass tubes for the preparation of tubular scaffolds were custom-ordered from Technical Glass Products (Painesville, USA). Phosphate-buffered saline (PBS), gelatin type B for cell culturing and Trypsin/EDTA were purchased from Sigma-Aldrich. All culture media components were ordered from Lonza.

\subsection{Synthesis and characterization of three-armed PTMC-MA}

Three-armed PTMC was synthesized by ring-opening polymerization of TMC at $130{ }^{\circ} \mathrm{C}$ for 2 days. In brief, $50.0 \mathrm{~g}(0.49 \mathrm{~mol})$ of TMC and $0.335 \mathrm{~g}(0.0025 \mathrm{~mol})$ of trimethylolpropane were added to a $250 \mathrm{~mL}$ three-necked round bottomed flask under argon atmosphere and the mixture was heated to $80{ }^{\circ} \mathrm{C}$ for $5 \mathrm{~min}$. To the melt $0.065 \mathrm{~g}$ of $\mathrm{Sn}(\mathrm{Oct})_{2}$ was added as a catalyst. The temperature was immediately raised to $130{ }^{\circ} \mathrm{C}$ and the mixture was maintained at this temperature for 2 days. After cooling to room temperature, the obtained three-armed PTMC was dissolved in $150 \mathrm{~mL}$ of dichloromethane, and $0.05 \mathrm{~g}$ of hydroquinone was added to prevent crosslinking during functionalization. To the solution of the three-armed PTMC $(0.0075 \mathrm{~mol}$ $\mathrm{OH}$ end groups $), 4 \mathrm{~mL}$ of MA $(0.027 \mathrm{~mol})$ and $3.5 \mathrm{~mL}$ of TEA $(0.025 \mathrm{~mol})$ were added. The reaction was conducted at room temperature under argon atmosphere for 5 days. The mixture was subsequently precipitated and washed in cold ethanol. The PTMC-MA was dried in a vacuum oven at $40{ }^{\circ} \mathrm{C}$ for 4 days. The average molecular weight $(\mathrm{Mn})$ and degree of 
functionalization of the three-armed PTMC and PTMC-MA were determined from ${ }^{1} \mathrm{H}-\mathrm{NMR}$ spectral data (Bruker Ascend 400/Avance III 400 MHz NMR spectrometer).

\subsection{Preparation and characterization of porous tubular scaffolds}

The PTMC-MA/NaCl composite resin was formulated as follows. PTMC-MA (10 g) was dissolved in $30 \mathrm{~g}$ of $\mathrm{PC}$ at $60{ }^{\circ} \mathrm{C}$ while stirring. After dissolution, Irgacure 2959 (2.5 wt\% relative to PTMC-MA) was added as a photo-initiator and the mixture was stirred for another $1 \mathrm{~h}$. Subsequently, dried $\mathrm{NaCl}$ particles $(80 \mathrm{~g})$ were added to the solution and the resulting mixture was stirred by hand using a spoon. The weight ratio of PTMC-MA:PC:salt was 1:3:8. Annular quartz glass molds, $80 \mathrm{~mm}$ in length and with $5 \mathrm{~mm}$ inner and $8 \mathrm{~mm}$ outer diameter were filled with the PTMC-MA/PC/salt resins. Silicone tubing ( $5 \mathrm{~mm}$ inner diameter, $1.5 \mathrm{~mm}$ wall thickness) was used to seal the two ends of the glass molds. The prepared samples were photo-crosslinked by UV $(365 \mathrm{~nm})$ at $8 \mathrm{~mW} / \mathrm{cm}^{2}$ light intensity for $2 \mathrm{~h}$. The molds were rotated 180 degrees each half hour during the photo-crosslinking process.

The inner tube of the mold was removed by hand. The outer tube was removed by swelling the photo-crosslinked resin in PC/ethanol (3:7 v/v) solution. Samples were extracted in PC/ethanol $(8: 2 \mathrm{v} / \mathrm{v})$ solution, after which ethanol was slowly dropped into the mixture until the PC content was below 5\%. Then, the solid photo-crosslinked PTMC-MA/NaCl tubes were soaked in ethanol to remove residual PC. After extraction, the resulting composites were dried at $50{ }^{\circ} \mathrm{C}$ for 12 hours. Subsequently, the constructs were soaked in distilled water for several days to leach out the $\mathrm{NaCl}$ particles. The obtained porous PTMC-MA scaffolds were stored in cold ethanol for future use.

The porosity of the tubular photo-crosslinked PTMC scaffolds was determined from gravimetric measurements and calculated according to equation 1 ,

Porosity $=[1-(\mathrm{W}$ dry $/ \mathrm{V}$ dry $* \rho \mathrm{PTMC})] * 100 \% \quad$ Eq 1

in which $\rho$ PTMC $=1.31 \mathrm{~g} / \mathrm{cm}^{3}$, W dry $=$ dry weight of the scaffold and $\mathrm{V}$ dry $=$ volume of the scaffold in dry condition (regarded as a solid tube). 
The pore size distribution of the scaffolds was determined from scanning electron microscopy (SEM) images. Cross-sections of scaffolds were sputtered with gold using a Cressington sputter coater 108 auto, and subsequently analyzed using a JSM-IT100 (JEOL Ltd. Japan). Average pore sizes were calculated based on 100 pores randomly taken from the SEM images. Adobe Photoshop software was employed to measure the pore size and calculate the average pore size.

\subsection{Culturing and seeding of SMCs}

SMCs isolated from human umbilical veins were cultured in $0.1 \%(\mathrm{w} / \mathrm{v})$ gelatin-coated cell culture flasks at $37^{\circ} \mathrm{C}$ and $5 \% \mathrm{CO}_{2}$ in an incubator. The culture medium consisted of $500 \mathrm{~mL}$ advanced DMEM supplemented with $25 \mathrm{~mL}$ FBS, $5 \mathrm{~mL}$ glutamax, $1 \mathrm{~mL}$ FGF- $\beta, 0.5 \mathrm{~mL}$ EGF, $0.5 \mathrm{~mL}$ insulin and $0.5 \mathrm{~mL}$ gentamycin/amphotericin B. Culture media were refreshed every 2 days. When cultures were almost confluent, SMCs were detached with $0.125 \%(\mathrm{w} / \mathrm{v})$ trypsin/0.05\% (w/v) EDTA and subcultured with a split ratio of 1:3 up to 10 passages.

Porous tubular PTMC scaffolds with a length of $4 \mathrm{~cm}$ were disinfected with $70 \%(\mathrm{v} / \mathrm{v})$ ethanol, rinsed with PBS, and coated with $0.1 \%(\mathrm{w} / \mathrm{v})$ gelatin for $1 \mathrm{~h}$ at $37^{\circ} \mathrm{C}$. SMCs were seeded in a scaffold by perfusing $10 \mathrm{~mL}$ of a SMC suspension in culture medium $\left(0.5 \times 10^{6}\right.$ cells $\left./ \mathrm{mL}\right)$ from the lumen into the wall with two syringes connected to both ends of the scaffold. During the first $2 \mathrm{~h}$, the seeded scaffolds were rotated $90^{\circ}$ every $10 \mathrm{~min}$ to ensure a homogeneous cell distribution. The cells were allowed to adhere to the scaffolds overnight in the incubator. Subsequently, cell culturing was continued under static conditions in the incubator or under dynamic conditions in a bioreactor (see below) up to 14 days. In the case of static culturing, culture media were refreshed every 3 days. 


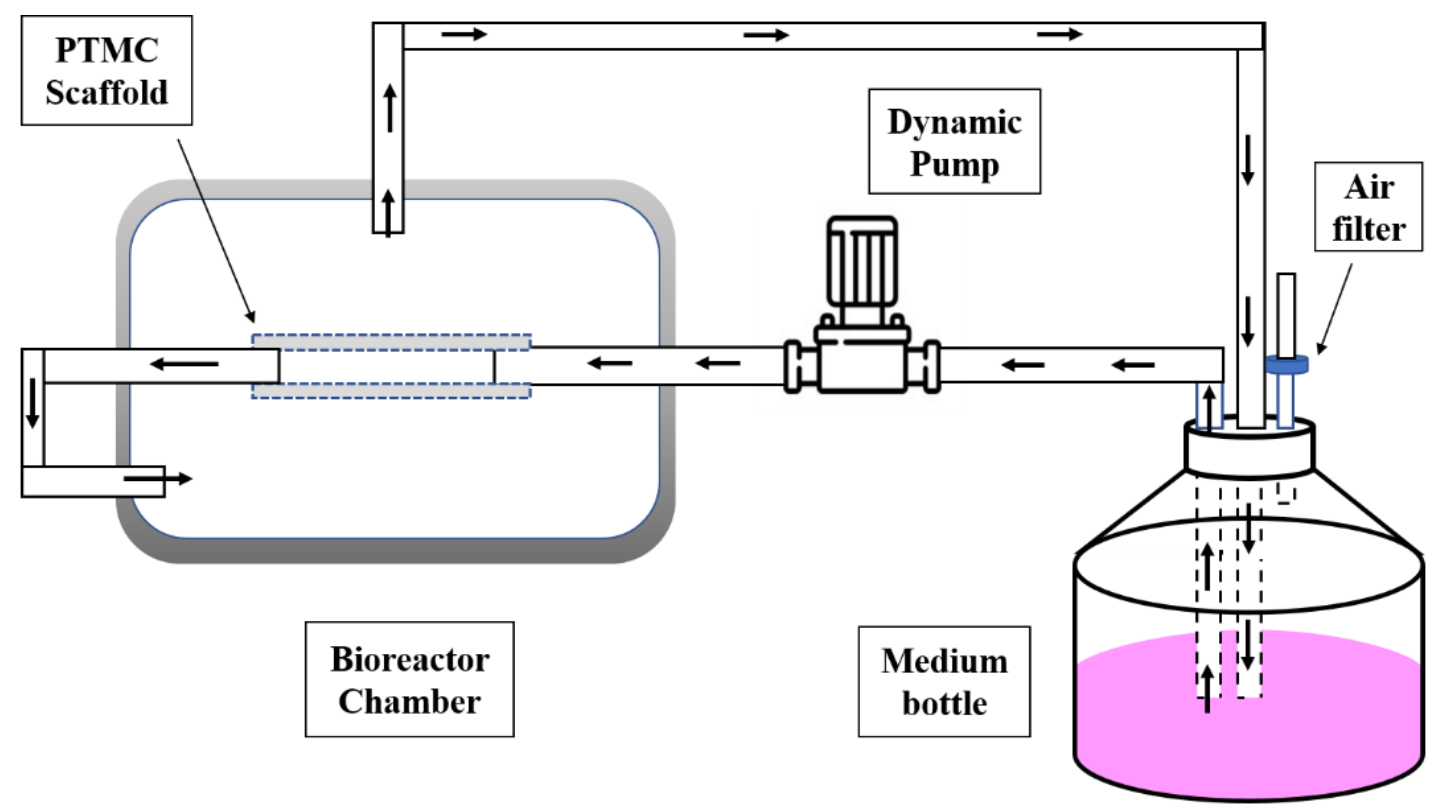

Scheme 1. Schematic representation of the pulsatile flow bioreactor.
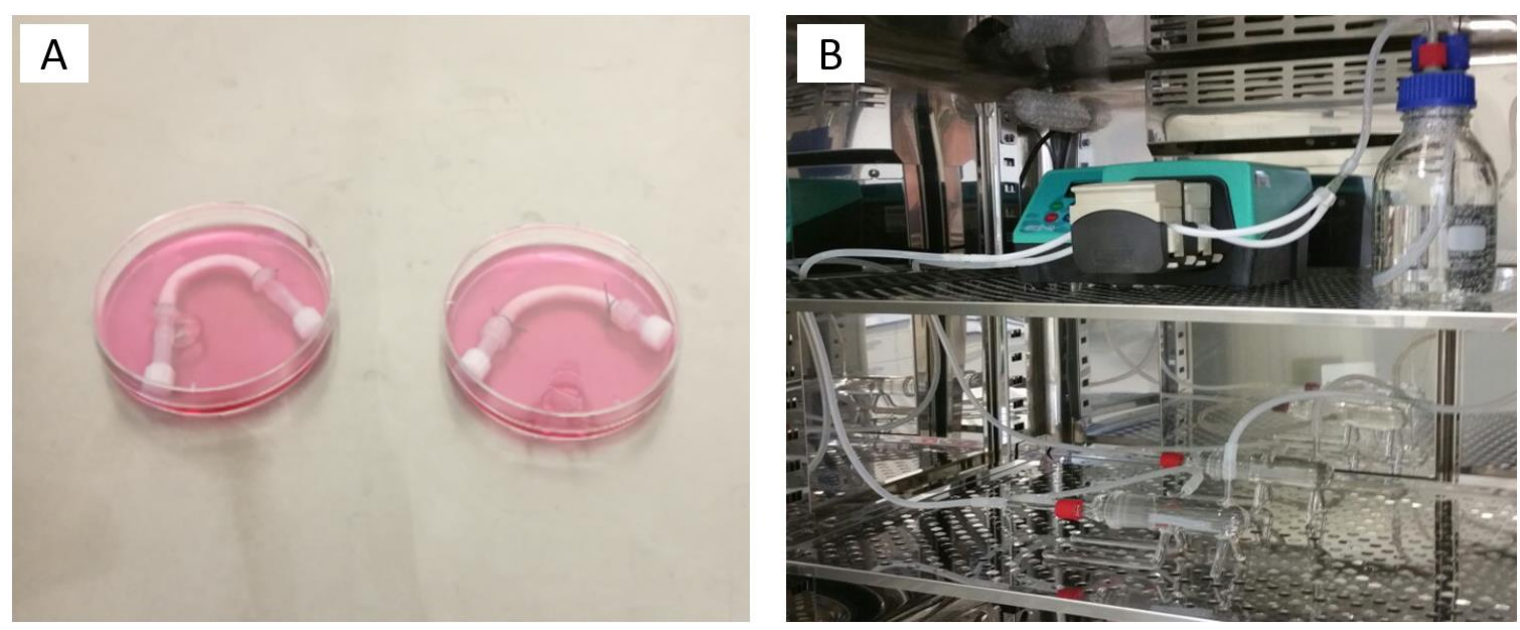

Figure 1. A: 3PTMC-MA scaffolds seeded with SMCs, B: bioreactor system in the incubator.

The SMC-seeded scaffolds were dynamically cultured in a pulsatile flow bioreactor (Scheme 1). Two culture chambers, each containing one construct, were operated in parallel. Culture media were perfused through the chambers using a dual head roller pump. The number of pulses was 90/min and the flow rate through each scaffold was $0.53 \mathrm{~mL} / \mathrm{s}$, resulting in a wall shear rate of $200 \mathrm{~s}^{-1}$. The bioreactor was placed in a $5 \% \mathrm{CO}_{2}$ incubator operating at $37^{\circ} \mathrm{C}$, see Figure 1. 


\subsection{Histological analysis}

PTMC-MA porous scaffolds seeded with SMCs and cultured for 14 days were rinsed with PBS. A construct was cut in 4 pieces of $8 \mathrm{~mm}$ length. One of these segments was fixed overnight in $4 \%(\mathrm{w} / \mathrm{v})$ paraformaldehyde solution, rinsed with demineralized water, and embedded in Jung tissue freezing medium (Leica). Transverse sections with a thickness of $20 \mu \mathrm{m}$ was cut at -22 ${ }^{\circ} \mathrm{C}$ using a microtome, stained with hematoxylin and eosin, and observed by light microscopy.

\subsection{CyQuant assay}

Numbers of SMCs present in the constructs were quantified by means of the CyQuant cell proliferation assay (Molecular Probes). A construct segment of $8 \mathrm{~mm}$ length was placed in 1 $\mathrm{mL} 0.1 \%(\mathrm{w} / \mathrm{v})$ Triton X-100 solution and frozen at $-20{ }^{\circ} \mathrm{C}$. After thawing and homogenizing the solution, $20 \mu \mathrm{l}$ was added to $180 \mu \mathrm{l}$ CyQuant dye solution, after which the fluorescence was measured in a Tecan plate reader at $480 \mathrm{~nm}$ excitation and $520 \mathrm{~nm}$ emission wavelengths.

\subsection{Tensile testing}

The mechanical properties of porous PTMC scaffolds and SMC-containing constructs in the hydrated state were determined in the radial direction using a Zwick Z020 tensile tester. Measurements were carried out according to standards of the American National Standards Institute and the Association for the Advancement of Medical Instrumentation (ANSI/AAMI VP20: 1994) at a tensile rate of $1 \mathrm{~mm} / \mathrm{min}$. The stiffness was determined from the slope of the tensile curve between 3 and $6 \%$ strain.

\subsection{Statistical analysis}

Differences between cell numbers as well as mechanical properties of constructs cultured under static and dynamic conditions were analyzed by Student's t-test, and considered significant when $\mathrm{p}<0.05$. 


\section{Results and discussion}

\subsection{Preparation and characteristics of the porous tubular scaffolds}

Three-armed PTMC with a molecular weight of $19 \mathrm{~kg} / \mathrm{mol}$ was synthesized by ring-opening polymerization of TMC using trimethylol as an initiator and stannous octoate as catalyst. Subsequent methacrylation using methacrylic anhydride in the presence of TEA afforded the PTMC-MA, in which $95 \%$ of the originally present hydroxyl groups were converted into methacrylate groups.

The prepared porous tubular scaffolds (Figure 2) had an inner diameter of $3.12 \pm 0.13 \mathrm{~mm}$, a wall thickness of $1.05 \pm 0.11 \mathrm{~mm}$, and a porosity of $73.3 \pm 2.1 \%$. From the SEM images an average pore size of $68 \pm 7 \mu \mathrm{m}$ was determined.
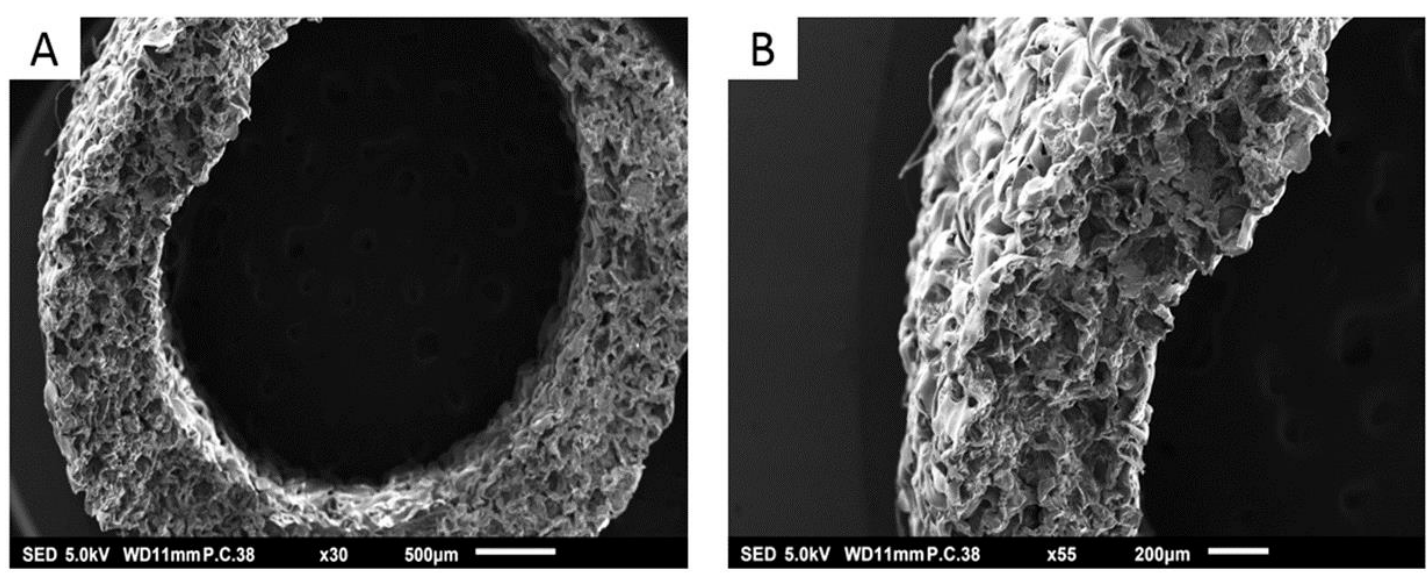

Figure 2. SEM images of a cross-section of a porous tubular PTMC-MA scaffold. A: scale bar is 500 m, B: scale bar is $200 \mu \mathrm{m}$.

The annular space of the mold had an inner and outer diameter of 5 and $8 \mathrm{~mm}$, respectively. The inner and outer diameter of the tubular PTMC scaffolds, however, were smaller. This difference can be explained by shrinkage of the scaffolds during post-processing. Photocrosslinking of the PTMC-MA macromers took place in the swollen state, due to the presence of PC, resulting in contraction of the PTMC network upon extraction of PC and subsequent salt leaching. Also, the length of the scaffolds decreased, from approximately $7 \mathrm{~cm}$ after photocrosslinking in the mold to $4.5 \mathrm{~cm}$ after post-processing (data not shown). 


\subsection{Seeding and culturing of SMCs in the scaffolds}

Figure 3 shows the results of the CyQuant cell proliferation assay after 14 days of culturing. SMC numbers in the dynamically cultured constructs were 3 times higher than in the constructs cultured under static conditions. This indicates that dynamic mechanical stimulation of the SMCs had a positive effect on proliferation of the cells. In addition, the higher cell numbers under dynamic conditions may be due to a more efficient nutrient and oxygen supply and waste product removal [17][22][23].

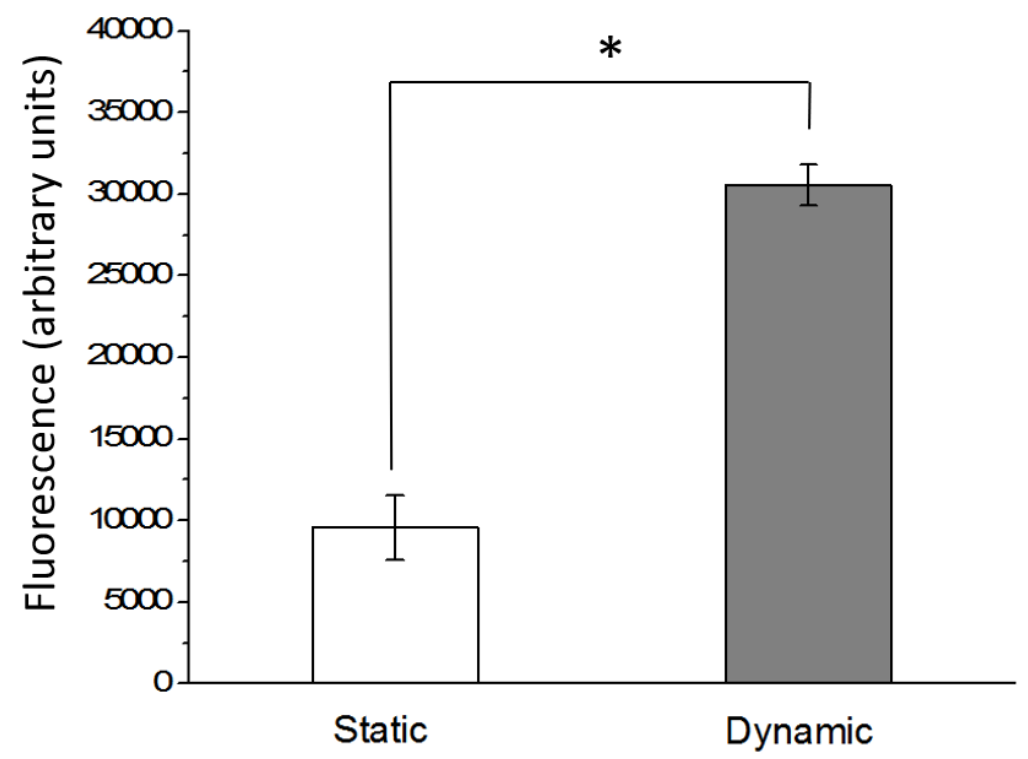

Figure 3. Numbers of SMCs present in the tubular PTMC scaffolds after 14 days of culturing. Data from CyQuant cell proliferation assay, $n=4 \pm S D .{ }^{*} p<0.001$.

Histological images of hematoxylin- and eosin-stained neat PTMC-MA scaffold and PTMCMA scaffolds cultured with SMCs for 14 days are shown in Figure 4. The porous structure of the neat PTMC-MA scaffold can be clearly observed because of dye absorption by the PTMCMA network. Comparing cell proliferation under static and dynamic culture conditions, it becomes clear that the pores were almost fully filled with cells when using dynamic conditions. 

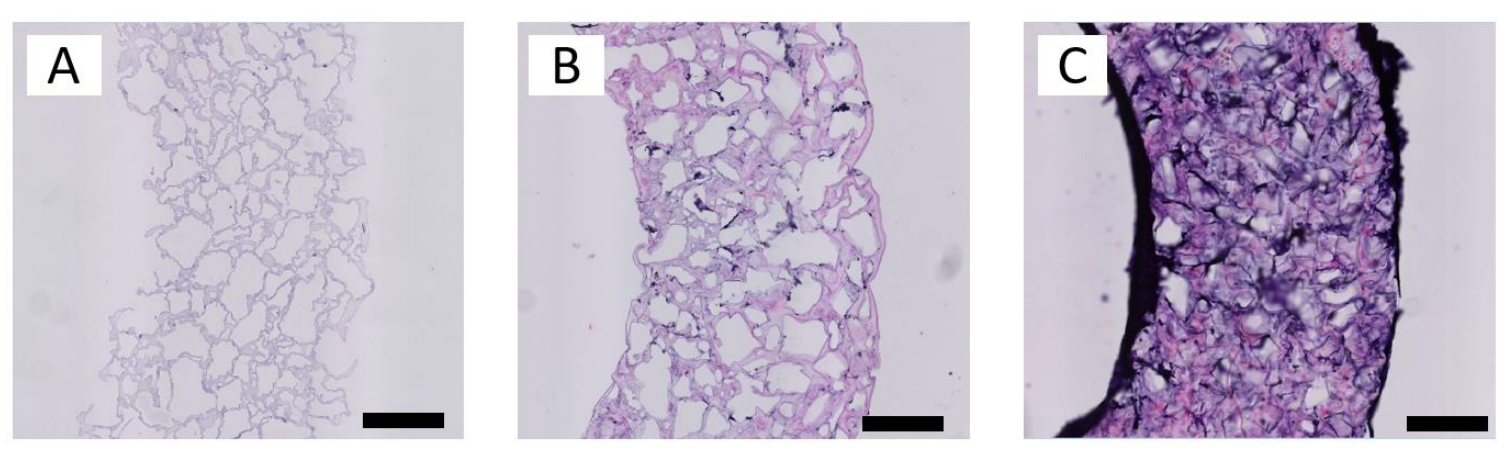

Figure 4. Cross-sections of hematoxylin- and eosin-stained PTMC-MA scaffolds. A: PTMC-MA scaffold, B: PTMC-MA scaffold with statically cultured cells, C: PTMC-MA scaffold with dynamically cultured cells. Scale bars are $200 \mu \mathrm{m}$.

\subsection{Mechanical properties of PTMC scaffolds cultured with SMCs}

The circumferential mechanical properties of the neat PTMC scaffold and scaffolds cultured with SMCs are listed in Table 1. The results show that compared to the neat PTMC scaffold, the elastic moduli of the SMC-cultured scaffolds using static and dynamic conditions were slightly higher and lower, respectively. The effect of dynamic culturing on the mechanical properties of the constructs is mainly reflected in the maximum stress. After culturing of SMCs in PTMC-MA scaffolds under pulsatile flow conditions, the maximum stress was significantly higher compared to static culture conditions. Combined with the histological data, these results can be ascribed to the deposition of new extracellular matrix. Collagen fibers in the extracellular matrix likely have a positive effect on the mechanical properties of the vascular graft [17].

Table 1. Tensile properties of 3PTMC-MA scaffolds cultured with SMCs $(n=4 \pm S D)$.

\begin{tabular}{lllll}
\hline Code & $\begin{array}{l}\text { Elastic } \\
\text { modulus, MPa }\end{array}$ & $\begin{array}{l}\text { Maximum } \\
\text { stress, MPa }\end{array}$ & Elongation, \% & Reference \\
\hline Scaffold & $0.21 \pm 0.01$ & $0.42 \pm 0.02$ & $2063 \pm 180$ & This work \\
\hline Static & $0.24 \pm 0.02$ & $0.45 \pm 0.03$ & $1989 \pm 160$ & This work \\
\hline Dynamic & $0.18 \pm 0.02$ & $0.61 \pm 0.05^{*}$ & $2190 \pm 210$ & This work \\
\hline $\begin{array}{l}\text { Gamma-irradiated } \\
\text { PTMC scaffold }\end{array}$ & $1.11 \pm 0.05$ & $0.47 \pm 0.06$ & $928 \pm 211$ & {$[17]^{\#}$} \\
\hline *p $<0.001$ compared to static. ${ }^{\#}$ Also cultured under dynamic conditions for 14 days with human SMCs.
\end{tabular}




\section{Conclusions}

A highly porous tubular PTMC scaffold was prepared by photo-crosslinking a PTMC/NaCl resin in a mold followed by particle leaching. Human SMCs were seeded in the scaffolds and culturing was performed under pulsatile flow conditions in a bioreactor for 14 days. Cell numbers and histology results revealed a significant improvement of SMC proliferation compared to the use of static culture conditions. Mechanical properties of the PTMC scaffolds cultured with SMCs under dynamic conditions were also significantly enhanced. Based on these results, and after endothelialization of the constructs, future in vivo implantation studies may show the potential of these vascular grafts for clinical application.

\section{References}

[1] H. Ma, J. Hu, P.X. Ma, Polymer scaffolds for small-diameter vascular tissue engineering, Adv. Func. Mater., 20 (2010) 2833-2841.

[2] http://www.healthline.com/health/peripheral-vascular-disease

[3] D.G. Seifu, A. Purnama, K. Mequanint, D. Mantovani, Small-diameter vascular tissue engineering, Nat. Rev. Cardiol., 10 (2013) 410.

[4] Y. Song, J. Feijen, D.W. Grijpma, A.A. Poot, Tissue engineering of small-diameter vascular grafts: a literature review, Clin. Hemorheol. Microcirc., 49 (2011) 357-374.

[5] N. Wang, W. Zheng, S. Cheng, W. Zhang, S. Liu, X. Jiang, In vitro evaluation of essential mechanical properties and cell behaviors of a novel polylactic-co-glycolic acid (PLGA)-based tubular scaffold for small-diameter vascular tissue engineering, Polymers, 9 (2017) 318.

[6] SB. Blanquer, S. Sharifi, D.W. Grijpma, Development of poly (trimethylene carbonate) network implants for annulus fibrosus tissue engineering, J. Appl. Biomater. Func., 10 (2012) 177-184.

[7] Z. Zhang, R. Kuijer, S.K. Bulstra, D.W. Grijpma, J. Feijen, The in vivo and in vitro degradation behavior of poly(trimethylene carbonate), Biomaterials, 27 (2006) 1741-1748.

[8] L. Yang, J. Li, W. Zhang, Y. Jin, J. Zhang, Y. Liu, D. Yi, M. Li, J. Guo, Z. Gu, The degradation of poly (trimethylene carbonate) implants: The role of molecular weight and enzymes, Polym. Degrad. Stab., 122 (2015) 77-87. 
[9] Z. Zhang, S. Zou, G.J. Vancso, D.W. Grijpma, J. Feijen, Enzymatic surface erosion of poly (trimethylene carbonate) films studied by atomic force microscopy, Biomacromolecules, 6 (2005) 3404-3409.

[10] S. Asadpour, H. Yeganeh, J. Ai, H. Ghanbari, A novel polyurethane modified with biomacromolecules for small-diameter vascular graft applications, Journal of Materials Science, 53 (2018) 9913-9927.

[11] P. Punnakitikashem, D. Truong, J.U. Menon, K.T. Nguyen, Y. Hong, Electrospun biodegradable elastic polyurethane scaffolds with dipyridamole release for small diameter vascular grafts, Acta Biomater., 10 (2014) 4618-4628.

[12] C.H. Mun, Y. Jung, S.H. Kim, S.H. Lee, H.C. Kim, I.K. Kwon, S.H. Kim, Threedimensional electrospun poly(lactide-co- $\varepsilon$-caprolactone) for small-diameter vascular grafts, Tissue Eng. Part A, 18 (2012) 1608-1616.

[13] H. Ahn, Y.M. Ju, H. Takahashi, D.F. Williams, J.J. Yoo, S.J. Lee, T. Okano, A. Atala, Engineered small diameter vascular grafts by combining cell sheet engineering and electrospinning technology, Acta Biomater., 16 (2015) 14-22.

[14] E. Yu, J. Zhang, J.A. Thomson, L.S. Turng, Fabrication and characterization of electrospun thermoplastic polyurethane/fibroin small-diameter vascular grafts for vascular tissue engineering, Int. Polym. Process, 31 (2016) 638-646.

[15] F. Kabirian, B. Ditkowski, A. Zamanian, R. Heying, M. Mozafari, An innovative approach towards 3D-printed scaffolds for the next generation of tissue-engineered vascular grafts, Mater. Today. Proceed, 5 (2018) 15586-15594.

[16] Y. Song, M.M. Kamphuis, Z. Zhang, L.T. Sterk, I. Vermes, A.A. Poot, J. Feijen, D.W. Grijpma, Flexible and elastic porous poly(trimethylene carbonate) structures for use in vascular tissue engineering, Acta Biomater, 6 (2010) 1269-1277.

[17] Y. Song, J.W. Wennink, M.M. Kamphuis, L.M. Sterk, I. Vermes, A.A. Poot, J. Feijen, D.W. Grijpma, Dynamic culturing of smooth muscle cells in tubular poly(trimethylene carbonate) scaffolds for vascular tissue engineering, Tissue Eng. Part A, 17 (2011) 381-387.

[18] Y. Song, J.W. Wennink, A.A. Poot, I. Vermes, J. Feijen, D.W. Grijpma, Evaluation of tubular poly(trimethylene carbonate) tissue engineering scaffolds in a circulating pulsatile flow system, Int. J. Artif. Organs, 34 (2011)161-171. 
[19] Y. Song, J.W. Wennink, M.M. Kamphuis, I. Vermes, A.A. Poot, J. Feijen, D.W. Grijpma, Effective seeding of smooth muscle cells into tubular poly(trimethylene carbonate) scaffolds for vascular tissue engineering, J. Biomed. Mater. Res. A, 95 (2010) 440-446.

[20] S. Schüller-Ravoo, J. Feijen, D.W. Grijpma, Flexible, elastic and tear-resistant networks prepared by photo-crosslinking poly(trimethylene carbonate) macromers. Acta Biomater, 8 (2012) 3576-3585.

[21] Z. Guo, DW. Grijpma, AA. Poot, Preparation and characterization of flexible and elastic porous tubular PTMC scaffolds for vascular tissue engineering, Polym. Adv. Technol, 28 (2017) 1239-1244.

[22] SL. Ishaug-Riley, G.M. Crane, A. Gurlek, M.J. Miller, A.W. Yasko, M.J. Yaszemski, A.G. Mikos, Ectopic bone formation by marrow stromal osteoblast transplantation using poly(DLlactic-co-glycolic acid) foams implanted into the rat mesentery, J. Biomed. Mater. Res., 36 (1997) 1-8.

[23] Freed L.E, G. Vunjak-Novakovic, Culture of organized cell communities, Adv. Drug Deliv. Rev., 33 (1998) 15-30. 


\section{Summary and outlook}

\section{Summary}

The past decades have shown major developments in biomedical engineering technologies, especially in tissue engineering and regenerative medicine. This poses a large demand on the preparation of suitable biomaterials for both research and industry. Among the different classes of biomaterials, polymer-based composites have received special attention in the past years. These composite materials can have several advantages related to presentation of functional bioactivity and improved mechanical properties, next to biocompatibility and biodegradability. The work presented in this $\mathrm{PhD}$ thesis introduces two applications of polymer-based composites. One is the use of electrically conductive reduced graphene oxide (rGO) fillers, enhancing the conductivity of scaffolds to be applied in nerve regeneration. Another is the use of sacrificial particle leaching, applied in the manufacturing of porous scaffolds for smalldiameter vascular tissue engineering.

Poly(trimethylene carbonate) (PTMC) was chosen as the polymer component, because of its excellent biocompatibility, surface erosion degradation behavior and flexible and elastic properties. Combination of the functional rGO filler with PTMC, was either done by mixing rGO in a PMTC matrix or grafting the polymer onto the rGO and subsequent mixing with PTMC. Porogens such as sodium chloride, sodium fluoride and calcium carbonate were used to fabricate porous scaffolds for vascular tissue engineering.

Next to a description of the material properties of the composites, also several porous scaffold manufacturing methods are described. These include sacrificial particle leaching, electrospinning and additive manufacturing. Moreover, in vitro and in vivo evaluation of prepared scaffolds were performed.

In Chapter 1, a general introduction on the background of the research described in this thesis is presented. The scope and outline of the studies are also introduced. In Chapter 2, the current state of the art on advancements in polymer-based composites, their properties, structure and fabrication techniques is presented. To engineer suitable scaffolds or implants for e.g. tissue 
engineering and regenerative medicine applications, structure property relationships of composites need to be considered. In this chapter, we focused on and discussed bioactive polymer-based composites, such as bone-forming, electrically conductive, magnetic, bactericidal and oxygen-releasing materials, and non-bioactive polymer-based composites containing reinforcing fillers and porogens. In addition, porous scaffold structures fabricated by particle leaching, electrospinning and additive manufacturing, all subjects of this $\mathrm{PhD}$ research, were described. It was concluded that current challenges are related to the limited choice of bioactive and functional fillers, control of the bioactive expression in the polymer matrix and manufacturing techniques for suitable structures. Therefore, studies on the relationship between the material properties of a specific polymer-based composite, its structure, physical and mechanical properties and biological response for a specific biomedical application remain necessary.

Previous studies on PTMC polymer technology developed within the research group, revealed the highly interesting properties of this material, like flexibility, elasticity and degradation without eliciting acidic degradation products in a biological environment. This material is therefore regarded highly suitable for the preparation of tubular scaffolds that can be applied as e.g. engineered nerve guides or blood vessel prostheses. In Chapter $\mathbf{3}$ a study on the preparation of $\mathrm{PTMC} / \mathrm{rGO}$ composites with electrical conductivity for peripheral nerve regeneration is described. rGO was obtained through reduction of graphene oxide (GO), which was prepared by a modified Hummers method. A 3-armed PTMC was synthesized by ringopening polymerization and end-functionalized with methacrylate groups. PTMC-MA/rGO composite films were subsequently prepared by mixing, solvent casting and photo-crosslinking. The PTMC-MA/rGO composite films with $0,0.5,1,2$ and $4 \mathrm{wt} \%$ rGO showed increasing electro-conductive properties with values up to $6.88 * 10^{-2} \mathrm{~S} / \mathrm{cm}$. Cell culturing with PC-12 cells showed that after initial cell adhesion, the cells proliferated on the surface of the PTMCMA/rGO composites, regardless of the rGO loading. These results provide evidence that composite materials of PTMC and rGO have promising properties for application as nerve guide conduits that accelerate nerve regeneration and were the basis for a study described in Chapter 4. In this chapter we describe a study on porous PTMC/rGO composite nerve guides, their preparation and in vivo evaluation. The composite nerve guide conduit with $2 \mathrm{wt} \% \mathrm{rGO}$ relative to PTMC was prepared by dip-coating, photo-crosslinking and leaching of sodium fluoride porogen particles. The conduit, which had a porosity of $73 \%$, was used to bridge a 15 $\mathrm{mm}$ sciatic nerve gap in a pilot study in rabbits. A porous PTMC conduit with the same porosity 
was used as a control. Macroscopic evaluation 6 weeks after implantation showed the formation of firm tissue in the PTMC/rGO nerve guide, whereas the tissue formed in the PTMC conduit lacked form stability. Histological analysis showed that the number of myelinated axons and the amount of collagen were higher in the PTMC/rGO nerve guide than in the PTMC conduit. The same differences were found in the respective distal nerve stumps.

To improve the compatibility and dispersion behavior of the rGO in the composites, an alternative strategy was chosen for the preparation of the composite material. In Chapter $\mathbf{5}$ a study on a new composite rGO-graft-PTMC is presented. rGO was synthesized by azido ethanol reaction with GO at a high temperature. The hydroxyl groups on the rGO were used to initiate the ring-opening polymerization of trimethylene carbonate monomer. The obtained rGO-graft-PTMC, comprising of single layer graphene nanosheets, afforded stable rGO-graftPTMC dispersions in chloroform. The rGO-graft-PTMC composites, with different PTMC molecular weights of 430-7030 $\mathrm{g} / \mathrm{mol}$, had electrical conductivities ranging from 0.2-0.016 $\mathrm{S} / \mathrm{cm}$. To investigate the biocompatibility of rGO-graft-PTMC, PTMC-based films containing rGO-graft-PTMC were prepared and used in cell culturing experiments. The composite films showed good biocompatibility with PC12 neuronal cells.

Another method than particle leaching to fabricate porous scaffolds is electrospinning. In Chapter 6, we describe a study on the preparation and properties of electrospun PTMC/rGOgraft-PTMC composite fibrous mats. A loading of the rGO-graft-PTMC in the PTMC matrix of up to $6 \mathrm{wt} \%$ could be reached. Scanning electron microscopy images showed that the morphology and average diameter of the PTMC/rGO-graft-PTMC composite fibers were affected by the content of rGO-graft-PTMC. Additionally, the incorporation of rGO-graftPTMC resulted in enhanced thermal stability and hydrophobicity of the PTMC-based fibers. Biological results demonstrated that PC12 cells showed higher cell viability on PTMC/rGOgraft-PTMC fibers of 2.4, 4.0 and $6.0 \mathrm{wt} \% \mathrm{rGO}$-graft-PTMC compared to pure PTMC fibers. The results of these studies suggest that PTMC/rGO-graft-PTMC composite structures hold great potential for neural tissue engineering.

In the last chapters and appendix of this thesis, our research on the engineering of porous scaffolds for small-diameter vascular tissue engineering is described. Due to the increasing prevalence of peripheral and cardiac vascular diseases, we focused on vascular grafts with an inner diameter of less than $6 \mathrm{~mm}$ for which there is an urgent need. Fabrication of branched micro-vascular channels for biomedical applications is presented in Chapter 7. $\mathrm{PTMC} / \mathrm{CaCO}_{3}$ 
composite films with various amounts of $\mathrm{CaCO}_{3}$ particles were prepared by solvent casting and photo-crosslinking. Particle leaching using a diluted $\mathrm{HCl}$ solution afforded micro-porous structures with porosities ranging from 33 to $71 \%$ and a pore size around $0.5 \mu \mathrm{m}$. A minimum $\mathrm{CaCO}_{3}$ content of 40 vol\% was required to get a porous structure with sufficient water permeation at a physiological pressure. The mechanical properties of the micro-porous films were similar to those of native blood vessels. To engineer complex vascular structures, photocrosslinkable PTMC/CaCO 3 composite resins were formulated for stereolithography-based additive manufacturing. We were able to build vascular scaffolds of various shapes and sizes with this $\mathrm{PTMC} / \mathrm{CaCO}_{3}$ composite resin. A very small branched vascular structure with a micro-porous wall, having a $482 \mu \mathrm{m}$ inner diameter, wall thickness of $146 \mu \mathrm{m}, 0.4 \mu \mathrm{m}$ pore size and 59\% porosity could be built, showing that complex structures and scaffolds for biomedical applications can be fabricated using this $\mathrm{PTMC} / \mathrm{CaCO}_{3}$ composite resin through additive manufacturing techniques.

In Chapter 8 we describe a method, based on salt leaching, for the manufacturing of porous tubular scaffolds for small-diameter vascular tissue engineering. Using a photo-crosslinkable composite resin composed of methacrylate end-functionalized PTMC macromer, sodium chloride as a sacrificial composite filler and propylene carbonate as a non-reactive diluent in a glass mold, porous structures were prepared after crosslinking, extraction of the diluent and particle leaching. Tubular scaffolds were obtained with an inner diameter of $3 \mathrm{~mm}$, a wall thickness of $1 \mathrm{~mm}$ and a length of $4.5 \mathrm{~cm}$. Pore sizes ranged from 0-290 $\mu \mathrm{m}$ and the porosity was around $70 \%$. The pores were homogeneously distributed and interconnected. With increasing PTMC macromer molecular weight from 4 to $22 \mathrm{~kg} / \mathrm{mol}$, the E-modulus and maximum tensile strength of the scaffolds in the radial direction increased from 0.56 to 1.12 $\mathrm{MPa}$ and 0.12 to $0.55 \mathrm{MPa}$, respectively. Stress-strain curves of scaffolds made from 13, 17 and $22 \mathrm{~kg} / \mathrm{mol}$ PTMC macromers, showed a 'toe' region characteristic for native arteries, followed by a linear increase until the maximum stress was reached. In view of their mechanical properties, porous tubular scaffolds made from PTMC macromers with a molecular weight of $13-22 \mathrm{~kg} / \mathrm{mol}$ appeared the preferred scaffolds for vascular tissue engineering.

In the Appendix we present an initial study on the culturing of seeded smooth muscle cells (SMCs) in porous tubular PTMC scaffolds under dynamic conditions in a pulsatile flow bioreactor. Scaffolds with a $3 \mathrm{~mm}$ inner diameter, $1 \mathrm{~mm}$ wall thickness and an average pore size of $68 \mu \mathrm{m}$ were used. SMCs cultured in porous PTMC scaffolds under static conditions was used for comparison. Cells were cultured for 14 days and a cell proliferation assay and 
histological evaluation were performed. A three times higher number of SMCs was found using dynamic culture conditions compared to static culturing. Histological images of the scaffolds cultured under dynamic conditions showed that the SMCs fully filled the porous scaffolds, which was not found after static culturing. Determination of the mechanical properties after dynamic culturing revealed an increase in the maximum stress of the constructs. It was concluded that dynamic culturing of cell-seeded PTMC scaffolds for vascular tissue engineering can be used for future in vivo experiments.

\section{Outlook and future perspectives}

Polymer-based composites can be regarded as a special class of materials and currently are widely investigated for the preparation of biomedical implants, grafts and other devices. Because polymer composites have advantages, such as low cost of available natural and synthetic polymers, filler types and ease and tunability of manufacturing techniques, it is envisaged that these materials will receive a prominent place in the biomedical field. It can be recognized that the combination of a specific polymer and composite filler will lead to novel material properties. Importantly, fillers not only can be used to change the material properties but can add bioactive properties to the composite. As an example, in the design of an implant the filler can be used to reinforce the polymer material but also be used to deliver bioactive components in a sustained manner. Manufacturing techniques may be used to create a 3D gradient scaffold structure, allowing a spatio-temporal regeneration of the targeted tissue.

As demonstrated in this thesis work, poly(trimethylene carbonate) (PTMC)-based composites with several kinds of functional fillers can be used for biomedical applications such as nerve regeneration conduits and vascular grafts. Several processing techniques were used to prepare those biomedical implants. Functional properties were added by using electrically conductive graphene oxides as fillers. Sacrificial particles were used to create porous tubular scaffolds allowing the diffusion of nutrients and waste products in the tissue regeneration process. While positive results were obtained in these studies, it can be concluded that improvements remain necessary for each application before such biomedical devices can be clinically applied. 


\subsection{Nerve guide conduits}

Due to trauma or disease nerves can be damaged and cause loss of function. The main function of a nerve guide conduit is to provide a temporary guide and protect the regenerating axons from invading cells from the environment, i.e. prevention of scar tissue formation. The wall of the conduit, however, should not be completely closed and allow the sustained delivery of nutrients and removal of cellular waste products. Particle leaching from polymer salt composites is an efficient way to make a porous structure. However, due to the limited access to of submicron leachable particles, porosity is generally not consistently small.

Electrically conductive materials are widely investigated for use in tissue engineering and regenerative medicine, especially in neural tissue engineering due to its bioactive electrical interface which could stimuli cells growing, differentiation and signaling. In addition, based on electrically conductive materials, electrostimulation could be applied to accelerated axonal growth and restoring nerve function. We showed that use of electrically conductive PTMC/rGO composite with a small amount ( $2 \mathrm{wt} \%$ relative to PTMC) of highly conductive rGO fillers for the fabrication of nerve guide conduits is a viable strategy to improve peripheral nerve regeneration.

It is envisaged that detailed in vivo experiments should be carried out with these PTMC/rGO composites to show their full potential. A complete nerve function characterization should be carried out to determine the threshold level for undisturbed movement. For future studies, it is recommended to increase the amount of in vivo experiments as well as the implantation time, and to include walking track analysis and electrophysiological measurements. To avoid in vivo implantation studies, the regeneration process may be evaluated using microfluidic techniques.

Although these conduits show promising results in the regeneration of nerves, their use was limited to small defects only. Development of improved conduits will likely focus on composite materials that also will deliver constituents like growth factors that stimulate the regeneration process. This may also stimulate the regeneration of large nerves and large size nerve defects (more than $3 \mathrm{~cm}$ ). 


\subsection{Porous vascular networks}

Capillary vascular networks are a necessary component of most native tissues and organs. This capillary structure provides the oxygen and nutrient supply for living cells as well as removal of waste products. To engineer tissues and organs for transplantation building of a vascular network plays an important role. Because the capillaries have a micro size inner diameter and wall thickness, these are difficult to fabricate. Selection of a suitable polymeric material, type of salt allows the preparation of scaffolds with sub-micro pore size of the wall and mechanical properties matching those of natural blood vessels. Such artificial vascular structures are needed for both traditional tissue engineering applications but also in studies on disease models. In this respect these materials hold great promise in their combination with microfluidic techniques. Functions, like platelet activation and adhesion dependent blood-graft interfaces may be studied. Such studies will contribute to optimal materials to be applied in blood-vessel tissue development.

\subsection{Small diameter vascular grafts}

Tissue engineering of small diameter vascular networks can have a significant impact on in vitro generated vascularized tissue and organ constructs for transplantation. 3D printing of vascular grafts recently received a lot of attention due to its efficiency and versatility in design. However, current 3D printing technologies do not allow the fabrication of highly porous scaffolds due to limitations of 3D printing devices and materials. Combining 3D printing, photo-crosslinking and particle leaching may well be a valuable step. Because mechanical stimulation has been shown to affect such processes tissue development using a bioreactor with pulsatile flow may stimulate tissue development. In combination with cell seeding techniques, future in vivo implantation studies may show the potential of these vascular grafts for clinical application.

\section{General conclusions}

We may conclude that bioactive or other functional fillers will endow biodegradable polymers large possibilities to be used in the repair or regeneration of tissues and other biomedical applications. The current techniques, applying polymer-based composites, in general need finetuning to optimize the structure required in the specific application. Polymer-based composite 
scaffolds already showed not only high cell adhesion, biocompatibility, and biodegradability but also bioactivities in terms of tissue formation, function, stimulation, survival and antibacterial properties during in vitro and in vivo experiments.

However, there are still challenges because of the limited choice of bioactive and functional fillers, control of the bioactive expression in the polymer matrix, and manufacturing techniques of suitable structures. Polymer-based composites should be adapted to the biological microenvironment, thereby accelerating tissue repair and regeneration. Studies on the relationship between the material properties of a specific poly(trimethylene carbonate-based composite, its structure, physical and mechanical properties and biological response for specific biomedical applications remain necessary. 


\section{Samenvatting}

In de laatste decaden zijn er belangrijke ontwikkelingen geweest in de biomedische technologie, met name in de weefselregeneratie (tissue engineering) en de regeneratieve geneeskunde (regenerative medicine). Hierdoor is er grote vraag naar de ontwikkeling van geschikte biomaterialen zowel voor gebruik in het onderzoek als in de industrie. Van de verschillende soorten biomaterialen, hebben op polymeer gebaseerde composieten de laatste jaren veel belangstelling gekregen. Gebruik van deze composiet materialen kan grote voordelen hebben, daar ze naast hun biocompatibiliteit en biodegradeerbaarheid verbeterde eigenschappen en functionele bioactiviteit kunnen presenteren. Het werk dat in dit proefschrift beschreven is introduceert twee toepassingen van polymeer composieten. De eerste is het gebruik van elektrisch geleidende gereduceerd grafeen oxide (reduced graphene oxide, $\mathrm{rGO}$ ) vulstoffen die de elektrische geleiding van cel- en weefsel dragermaterialen (tissue engineering scaffolds) in zenuwregeneratie verbeteren. In een tweede toepassing worden oplosbare partikels gebruikt om poreuze scaffolds voor de regeneratie van kleine-diameter bloedvaatjes te maken.

Poly(trimethyleen carbonaat) (PTMC) werd gekozen voor de polymere component, gezien de uitstekende biocompatibiliteit, oppervlakte erosie degradatiegedrag en flexibele en elastische eigenschappen. Het combineren van de functionele rGO vulstof met PTMC werd gedaan door rGO in een PTMC matrix in te mengen, of door het enten van het polymeer op het rGO gevolgd door het mengen met PTMC. Porie-vormende verbindingen als natrium chloride, natrium fluoride en calcium carbonaat werden gebruikt voor het maken van poreuze scaffolds voor tissue engineering van bloedvaten. Naast het beschrijven van de materiaal eigenschappen van de composieten, worden ook verschillende fabricagemethoden voor het maken van poreuze scaffold structuren besproken. Deze omvatten methoden gebaseerd op het uitwassen van deeltjes, electrospinnen en additieve fabricage technieken (additive manufacturing). Ook zijn de gemaakte scaffolds in vitro en in vivo geëvalueerd.

In Hoofdstuk 1 wordt een algemene inleiding in de achtergrond van het onderzoek dat in dit proefschrift is beschreven gegeven. Ook wordt ingegaan op het belang van het onderzoek en wordt een overzicht van de uitgevoerde studies gegeven. In Hoofdstuk 2 wordt de huidige kennis en technologie op het gebied van polymeer composieten en hun eigenschapen, en verschillende methoden om ze te bereiden, gepresenteerd. Om geschikte scaffolds of implantaten voor bijvoorbeeld tissue engineering en regeneratieve geneeskunde te ontwerpen, 
moeten de structuur-eigenschap relaties van composieten in beschouwing worden genomen. In dit hoofdstuk hebben we ons gericht op bioactieve polymeer composieten zoals botvormende-, elektrisch geleidende-, magnetische-, bacteriedodende en zuurstof-afgevende materialen, en op niet-bioactieve polymere composieten die versterkende vulstoffen of uitwasbare partikels bevatten. Daarnaast worden poreuze scaffolds gemaakt door het uitwassen van deeltjes, electrospinnen en additive manufacturing, allemaal onderwerpen die onderzocht zijn in dit promotieonderzoek, beschreven. Er werd geconcludeerd dat de huidige uitdagingen verband houden met de beperkte keuze aan bioactieve en functionele vulstoffen, de expressie van bioactiviteit in de polymere matrix en de fabricage technieken van geschikte structuren. Daarom zijn studies die een verband leggen tussen de materiaal eigenschappen van een specifiek polymeer composiet, zijn structuur, zijn fysische en mechanische eigenschappen en de biologische respons in een bepaalde biomedische toepassing, van groot belang. Eerdere studies in onze onderzoeksgroep naar PTMC lieten de zeer interessante eigenschappen van dit materiaal zien: bijvoorbeeld zijn flexibiliteit, elasticiteit, en degradatiegedrag in een biologische omgeving waarbij zure producten niet gevormd worden. Dit materiaal kan daarom als zeer geschikt worden beschouwd voor het maken van buisvormige scaffolds voor toepassing als bijvoorbeeld regenererende zenuwgeleiders of bloedvatprothesen.

In Hoofdstuk 3 wordt een studie naar de bereiding van elektrisch geleidende PTMC/rGO composieten voor de regeneratie van perifere zenuwen beschreven. rGO werd verkregen door reductie van grafeen oxide (GO), dat bereid werd volgens de gewijzigde methode van Hummer.

3-armig PTMC werd gesynthetiseerd door ring-openingspolymerisatie en eind-functionalisatie met methacrylaat groepen. Composieten van PTMC-MA/rGO in film vorm werden vervolgens gemaakt door mengen, gieten uit oplossen en vernetten onder invloed van licht (photocrosslinking). De PTMC-MA/rGO composiet films met 0, 0.5, 1, 2 en 4 gewichts \% rGO lieten toenemende elektrische geleiding zien met waarden tot $6.88^{*} 10^{-2} \mathrm{~S} / \mathrm{cm}$. Celkweekexperimenten met PC-12 cellen lieten zien dat na initiële celadhesie de cellen onafhankelijk van de rGO belading op het oppervlak van de PTMC-MA/rGO composieten prolifereerden. Deze resultaten laten de gunstige eigenschappen van PTMC en rGO voor toepassing als zenuwgeleiders die regeneratie van zenuwen kunnen versnellen zien. Zij zijn de basis voor de vervolgstudie die in Hoofdstuk 4 gepresenteerd wordt.

In dit hoofdstuk beschrijven we een studie naar poreuze composiete PTMC/rGO zenuwgeleiders, hun bereiding en evaluatie in vivo. De composiete zenuwgeleider met 2 
gewichts \% rGO (in relatie tot PTMC) werd bereid door dompelen in een polymeeroplossing (dipcoating), photo-crosslinking en uitwassen van natrium fluoride porie-vormende partikels. De geleider, die een porositeit van $73 \%$ had, werd in een proefonderzoek gebruikt voor het overbruggen van een $15 \mathrm{~mm}$ defect in de heupzenuw van konijnen. Een poreuze PTMC geleider met dezelfde porositeit werd als controle gebruikt. Macroscopische evaluatie 6 weken na implantatie liet de vorming van stevig weefsel in de PTMC/rGO zenuwgeleider zien, terwijl het weefsel dat in de PTMC geleider gevormd was niet vormvast was. Histologische analyse liet zien dat het aantal gemyelineerde axonen en de hoeveelheid collageen in de PTMC/rGO zenuwgeleider groter was dan in de PTMC geleider. Dezelfde verschillen werden ook gevonden in de respectievelijke distale zenuwstompen.

Om de compatibiliteit en het dispersiegedrag van het rGO in de composieten te verbeteren, werd een alternatieve strategie gekozen voor de bereiden van de composiet materialen. In Hoofdstuk 5 wordt een studie naar een nieuw composiet rGO-ent-PTMC gepresenteerd. rGO werd gesynthetiseerd door de reactie van azido ethanol met rGO bij hoge temperatuur. De aanwezige hydroxyl groepen op het rGO worden dan gebruikt voor de initiatie van de ringopeningspolymerisatie van het trimethyleen carbonaat monomeer. Het verkregen rGO-entPTMC bestaat uit enkel-laags grafeen nano-vellen en geeft stabiele rGO-ent-PTMC dispersies in chloroform. De rGO-ent-PTMC composieten, met PTMC moleculaire massa's van 430$7030 \mathrm{~g} / \mathrm{mol}$, hadden elektrische geleidbaarheden variërend van 0.2-0.016 S/cm. Om de biocompatibiliteit van rGO-ent-PTMC te onderzoeken, werden films van PTMC die rGO-entPTMC bevatten gemaakt en in celkweek experimenten gebruikt. De composiete films lieten goede biocompatibiliteit met neurale PC12 cellen zien.

Een andere manier dan het uitwassen van partikels om poreuze scaffolds te maken is electrospinnen. In Hoofdstuk 6 beschrijven we een studie naar het maken van vezelachtige matten van PTMC/rGO-ent-PTMC composieten door electrospinnen en hun eigenschappen. Een belading van 6 gewichts\% van het rGO-ent-PTMC in de PTMC matrix kon bereikt worden.

Raster elektronen microscopie beelden lieten zien dat de morfologie en de gemiddelde diameter van de PTMC/rGO-ent-PTMC composiete vezels beïnvloed werd door de hoeveelheid rGOgraft-PTMC. Ook resulteerde de incorporatie van rGO-ent-PTMC in toename van de thermische stabiliteit en hydrofobiciteit van op PTMC gebaseerde vezels. Biologische resultaten lieten zien dat de levensvatbaarheid van PC12 cellen hoger was op PTMC/rGO-entPTMC vezels van 2.4, 4.0 en 6.0 wt\% rGO-ent-PTMC in vergelijking met puur PTMC vezels. 
De resultaten van deze studie suggereren dat structuren gebaseerd op PTMC/rGO-ent-PTMC zeer geschikt zijn voor gebruik in de regeneratie van neuraal weefsel.

In de laatste hoofdstukken en appendix van dit proefschrift wordt ons onderzoek naar de ontwikkeling en vervaardiging van poreuze scaffolds voor tissue engineering van kleinediameter bloedvaten beschreven. Door het toenemend voorkomen van perifere vaat- en cardiovasculaire ziekten, hebben we ons gericht op bloedvatprothesen met een inwendige diameter kleiner dan $6 \mathrm{~mm}$ waar grote behoefte aan is.

Het maken van vertakte micro-vasculaire kanalen voor biomedische toepassingen wordt in Hoofdstuk 7 gepresenteerd. Composiete $\mathrm{PTMC} / \mathrm{CaCO}_{3}$ films met verschillende hoeveelheden $\mathrm{CaCO}_{3}$ deeltjes werden gemaakt door te gieten uit oplossing en foto-crosslinken. Het uitwassen van de deeltjes met een verdunde $\mathrm{HCl}$ oplossing gaf micro-poreuze structuren met porositeiten van 33 tot $71 \%$ en porie afmetingen van circa $0.5 \mu \mathrm{m}$. Een gehalte van minimaal 40 volume \%

$\mathrm{CaCO}_{3}$ was nodig om een poreuze structuur met voldoende waterpermeabiliteit bij fysiologische druk te verkrijgen. De mechanische eigenschappen van de micro-poreuze films waren vergelijkbaar met die van natuurlijke bloedvaten. Om complexe vasculaire structuren te kunnen maken, werden foto-vernetbare $\mathrm{PTMC} / \mathrm{CaCO}_{3}$ composiete hars samenstellingen geformuleerd voor stereolithografie. We waren in staat vasculaire scaffolds van verschillende vormen en afmetingen te maken met deze PTMC/CaCO${ }_{3}$ harsen. Een zeer kleine vertakte vasculaire structuur met micro-poreuze wanden kon worden gebouwd. Van deze structuur was de binnen diameter $482 \mu \mathrm{m}$, de wanddikte $146 \mu \mathrm{m}$, de poriegrootte $0.4 \mu \mathrm{m}$ en de porositeit 59\%. Dit laat zien dat het mogelijk is om complexe structuren en scaffolds voor biomedische toepassingen met deze $\mathrm{PTMC} \mathrm{CaCO}_{3}$ composiete harsen te maken middels additieve fabricagetechnieken.

In Hoofdstuk 8 beschrijven we een methode gebaseerd op het uitwassen van zout deeltjes voor het maken van buisvormige scaffolds voor tissue engineering van kleine-diameter bloedvaatjes.

Door gebruik te maken van een foto-vernetbare composiete hars samengesteld uit methacrylaat eind-gefunctionaliseerd PTMC macromeer, natrium chloride als oplosbare vulstof en propyleen carbonaat als niet-reactive verdunner in een glazen matrijs, konden poreuze structuren verkregen worden na crosslinking, extractie van de verdunner en uitwassen van de vulstof deeltjes. Buisvormige scaffolds met een binnen diameter van $3 \mathrm{~mm}$, een wanddikte van $1 \mathrm{~mm}$ en een lengte van $4.5 \mathrm{~cm}$ werden verkregen. De porieafmetingen varieerden van 0-290 $\mu \mathrm{m}$ en de porositeit was circa $70 \%$. De poriën waren homogeen verdeeld en met elkaar 
verbonden. Met toenemend molecuulgewicht van het PTMC macromeer van 4 tot $22 \mathrm{~kg} / \mathrm{mol}$, namen de E-modulus en de maximale treksterkte in de radiële richting toe van, respectievelijk, 0.56 tot $1.12 \mathrm{MPa}$ en van 0.12 tot $0.55 \mathrm{MPa}$. Spannings-rek curven van scaffolds gemaakt van PTMC macromeren met een molecuulgewicht van 13, 17 en $22 \mathrm{~kg} / \mathrm{mol}$ vertoonden een "teen" gebied dat karakteristiek is voor natuurlijke arteriën, gevolgd door een lineaire toename tot de maximale spanning werd bereikt. Voor wat hun mechanische eigenschappen betreft, lijken poreuze buisvormige scaffolds gemaakt van PTMC macromeren met molecuulgewichten van $13-22 \mathrm{~kg} / \mathrm{mol}$ de meest geschikte scaffolds voor bloedvat tissue engineering.

In de Appendix presenteren we een initiële studie naar het kweken van gezaaide gladde spiercellen (smooth muscle cells, SMCs) in poreuze buisvormige PTMC scaffolds onder dynamische omstandigheden in een bioreactor met pulsatiele vloeistofstroom. Scaffolds met een binnen diameter van $3 \mathrm{~mm}$, een wanddikte van $1 \mathrm{~mm}$ en een gemiddelde poriegrootte van $68 \mu \mathrm{m}$ werden gebruikt. SMCs gekweekt in poreuze PTMC scaffolds onder statische condities werden ter vergelijking gebruikt. De cellen werden gedurende 14 dagen gekweekt, waarna cel proliferatie bepaald werd en histologische evaluaties gedaan werden. Het aantal SMCs gekweekt onder dynamische condities was driemaal zo hoog als die gekweekt onder statische condities. Histologische beelden van scaffolds gekweekt onder dynamische condities lieten zien dat de SMCs de poreuze scaffolds volledig vulden, dit was niet het geval na statisch kweken. Bepalingen van de mechanische eigenschappen na dynamisch kweken lieten een toename van de maximale treksterkte van de constructen zien. Er werd geconcludeerd dat dynamisch kweken van met cel-gezaaide PTMC scaffolds voor bloedvat tissue engineering gebruikt kan worden in toekomstige in vivo experimenten. 


\section{摘要}

在过去的二十年里, 生物医学工程技术有了很大的发展, 特别是在组织工程和再 生医学领域。这导致了制备合适生物材料的巨大需求在研究领域和工业领域。在不同 种类的生物材料里，高分子基复合材料在近几年得到了很大的关注。高分子基复合材 料有着以下几个方面的优势, 例如复合材料可以提供功能化的生物活性, 可改善的力 学性能, 以及较好的生物相容性和生物可降解性。导电性的还原氧化石墨烯填料可以 增加生物支架的电导率进而应用于神经修复领域; 另一个例子就是利用可洗出的颗粒 制备多孔性的管状支架用于小直径血管组织工程研究。

聚三甲基碳酸脂（PTMC）被选择用来作为复合材料的高分子组分, 理由是其优 异的生物相容性, 表面降解特性, 可拉伸以及回弹性。PTMC 和还原氧化石墨烯（rGO） 的混合分别用了直接溶液搅拌混合, 接枝 PTMC 在还原氧化石墨烯表面然后再混合两 种方式。制孔剂氯化钠、氟化钠和碳酸钙被用来制备小直径人造血管组织工程的多孔 性支架。

接下来论文介绍了高分子基复合材料的力学性能, 以及几种制备多孔性支架的方 法。包括颗粒洗出, 静电纺丝和增材制造。并进一步对制备的支架进行了体内和体外 实验评估。

在第一章中, 对整个论文的研究背景做了介绍。对论文研究的范围和目的也进行 了介绍。第二章介绍了高分子基复合材料的先进性, 性质, 结构和制备方法。为组织 工程和再生医学制造可实用性的支架或者植入物, 复合材料的结构性质和性质关系需 要被考虑。在本章中, 我们重点关注和讨论了生物活性的高分子基复合材料, 像骨再 生性, 导电性, 磁性, 灭菌性和氧气活性; 以及非生物活性高分子基复合材料包含增 强性填料和制孔剂。此外, 颗粒析出, 静电纺丝, 和增材制造等制备多孔性支架结构 的方法在博士论文中也做了描述。得出结论的是目前的挑战包括, 缺少足够种类的生 物活性和功能性的填料, 控制生物活性在高分子母体中的表达, 以及合适结构的制备 技术。因此, 研究高分子基复合材料性质, 结构, 物理, 力学性能, 和生物反应之间 的关系对特定生物医学的应用是十分必要的。 
之前对于 PTMC 的研究, 在本课题组高分子技术的发展, 揭示了这种材料非常有 意思的特性, 比如延展性, 弹性和没有酸降解产物生物环境降解性。这种材料因此被 认为是制备管能够应用于制造像神经导管或者血管植入物的管状支架。在第三章中描 述了一个关于制备 $\mathrm{PTMC} / \mathrm{rGO}$ 导电性复合材料被用于周围神经修复。通过还原由改进 的 Hummers 方法得到的氧化石墨烯 (GO), 制备出了 rGO。一种三臂的 PTMC 被通 过开环聚合的方法合成出来, 进而用甲基丙烯酸酯基对其进行了末端功能化。紧接着 PTMC-MA/rGO 复合材料膜通过搅拌, 溶剂浇铸和光交联的方法制备出来。含有 0 ,

$0.5,1,2$ 和 $4 \%$ 还原氧化石墨烯的 PTMC/rGO 复合材料膜的导电性逐渐升高至 $6.88 * 10^{-}$ ${ }^{2} \mathrm{~S} / \mathrm{cm}$ 。 PC-12 小鼠神经细胞培养实验表明细胞初次黏附后, 细胞增殖在 $\mathrm{PTMC} / \mathrm{rGO}$ 复 合材料表面跟 rGO 的含量无关。这些结果提供了 PTMC/rGO 复合材料具有优异的性质 能够作为神经导管促进神经修复证据和第四章的研究作为一个基础。在此章节中, 我 们阐述了多孔性 PTMC/rGO 复合材料神经导管的研究，其制备和体内评估。含有 $2 \mathrm{wt} \%$ rGO 相对于 PTMC 质量的复合材料神经导管通过浸涂, 交联和洗出氟化钠颗粒的方法 被制备出来。此导管, 孔隙率 $73 \%$, 被用于连接 $15 \mathrm{~mm}$ 兔子坐骨神经缺损的初步实验。 含有相同孔隙率的多孔性 PTMC 导管被用来作为空白实验。植入 6 周后显微评估表明 在 PTMC/rGO 神经导管生成了硬组织, 相对而言在 PTMC 导管中形成的组织不具备此 性质。组织学分析显示髓鞘轴突和胶原含量, PTMC/rGO 神经导管多于 PTMC 导管。 这种不同也发现于远端神经。

为了改善 $\mathrm{rGO}$ 在复合材料中的相容性和分散性, 另一种替代策略被选择作为制备 此类复合材料。在第五章中, 研究了新的 rGO-graft-PTMC 复合材料。通过高温下, 氧 化石墨烯和叠氮乙醇的反应来合成还原氧化石墨烯。在石墨烯表面的羟基基团用来引 发三甲基碳酸脂单体的开环聚合反应。这种得到的氧化石墨烯接枝聚三甲基碳酸脂包 含的是单层石墨烯纳米片, 能够使 rGO-graft-PTMC 在氯仿中稳定的分散。这种 rGOgraft-PTMC 中 PTMC 的分子量是 430-7030g/mol, 导电性从 0.2 到 $0.016 \mathrm{~S} / \mathrm{cm}$ 。为了探 讨 rGO-graft-PTMC 的生物相容性, 制备了 PTMC/rGO-graft-PTMC 膜并在其表面进行 了细胞培养。复合材料膜对 PC12 神经细胞显示出了较好的生物相容性。

相较于颗粒洗出, 另一种方法静电纺丝也能制备多孔性支架。在第六章中, 我们 描述了静电纺丝 PTMC/rGO-graft-PTMC 复合材料纤维膜的制备和性能。rGO-graftPTMC 在 PTMC 中的含量可以达到 6wt\%。扫描电镜图像显示 rGO-graft-PTMC 含量会 
影响 PTMC/rGO-graft-PTMC 复合材料的表面形貌和平均直径。此外, rGO-graft-PTMC 参杂使用会增加 PTMC 纤维的热稳定性和疏水性。生物实验结果证明 PC12 细胞在含有 2.4, 4.0 and $6.0 \mathrm{wt} \%$ rGO-graft-PTM 的 PTMC/rGO-graft-PTMC 纤维上相较于纯 PTMC 纤 维上显示出了更高的细胞活性。这些结果显示 PTMC/rGO-graft-PTMC 复合材料结构在 神经组织工程上有着巨大的应用潜力。

在这本论文后面的章节和附录中, 我们描述了制造小直径血管组织工程的多孔性 支架研究。因为周围和心脏血管疾病的患病率的增加, 我们主要关注当前急需的内径 小于 $6 \mathrm{~mm}$ 的血管移植体。用于生物医学的分支型微血管管道的制备被放在了第七章。

多种 $\mathrm{CaCO}_{3}$ 含量的 $\mathrm{PTMC} / \mathrm{CaCO}_{3}$ 复合材料膜通过溶剂浇铸和光交联被制备出来。使用 稀释盐酸溶液颗粒洗出得到了孔隙率在 $33-71 \%$ 和孔径在 0.5 微米的微孔结构。得到在 生理压力下具有足够水通过率的多孔结构需要至少 $40 \%$ 的 $\mathrm{CaCO}_{3}$ 。微孔结构膜的力学 性能和天然血管的力学性能相似。为了制造复杂的血管结构, 配制出了可光固化的 $\mathrm{PTMC} / \mathrm{CaCO}_{3}$ 复合材料树脂用于立体光刻技术的增材制造。用这种 $\mathrm{PTMC} / \mathrm{CaCO}_{3}$ 复合 材料树脂我们可以打印多种形状和尺寸的血管支架。一个非常小的带有微孔壁的分支 血管结构, 内径 482 微米, 壁厚 146 微米, 空尺寸 0.4 微米和孔隙率 59\%, 可以被制备

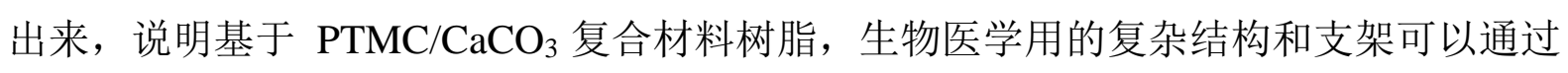
增加制造方法制备出来。

在第八章, 我们介绍了一种方法, 基于盐颗粒洗出, 用于制造小直径血管组织工 程的多孔性管状支架。用甲基丙烯酸酯末端官能化的 PTMC 大分子单体, 氯化钠作为 洗出复合材料填料以及非反应性稀释剂碳酸丙烯酯在一个玻璃模具中, 在交联, 抽提 稀释剂和颗粒洗出后, 制备出了多孔性结构。管状支架内径 $3 \mathrm{~mm}$, 壁厚 $1 \mathrm{~mm}$, 长度 $4.5 \mathrm{~cm}$ 。孔尺寸范围在 0-290mm, 孔隙率 70\%左右。这些孔是均匀分布且相互贯通的。 增加 PTMC 大分子的分子量, 从 4-22kg/mol, 其制备出支架的径向杨氏模量和最大拉 伸应力逐渐增加, 分别为 0.56-1.12 兆帕和 0.12 到 0.55 兆帕。从 13,17 到 $22 \mathrm{~kg} / \mathrm{mol}$ 分 子量的 PTMC 大分子制备出支架的应力应变曲线, 显示出了一个符合自体动脉的 “脚 趾” 区特性, 紧随其后的是一直到最大应力的线性增加。总的来看由 $13-22 \mathrm{~kg} / \mathrm{mol}$ 分子 量 PTMC 大分子制备出的管状支架的力学性能显示出了其可以用于血管组织工程。

在附录中, 我们使用脉动生物反应器对种植过平滑肌细胞 (SMCs) 的多孔管状 PTMC 支架做了动态培养细胞增殖。使用了内径为 $3 \mathrm{~mm}$, 壁厚 $1 \mathrm{~mm}$, 平均孔径为 
$68 \mathrm{~mm}$ 的支架。对比实验是静态培养 SMCs 种植的多孔性 PTMC 支架。细胞培养了 14 天, 之后进行了进行细胞增殖测定和组织学评估。动态培养得到 SMCs 细胞数量 3 倍 于静态培养。组织学图片显示, 在动态培养下, SMCs 细胞完全填满了多孔支架, 而 静态培养则没有。通过测试动态培养后支架的力学性能发现, 其最大拉伸应力得到了 提高。总的来说, 动态培养种植细胞后的 PTMC 血管组织工程支架在未来可以用于体 内实验。 


\section{Acknowledgements}

Since 2014, I received CSC scholarship and studied in The Netherlands. It was 6 years ago; I still remember the first time I arrived Schiphol and took the train to Enschede together with Jia and Huiyu Yuan. I was so excited When I looked through the window. I was first in foreign country and thinking how I could live in this place. Time fly, 6 years has been passed and I am back to China more than half year. When I am looking back the time I spend in The Netherlands, I should appreciate many many people I meet. Without you, I could not have a chance to study in University of Twente and enjoy a colorful life in Enschede.

First, I appreciate Prof. Dirk Grijpma. I can only have this chance through him to study and learn what I like such as polymer network and 3D printing after my master study. He replied me so quickly and forwarded my application to Prof. André Poot. In the next 4 years, he pointed a lot things I need to improve, and I learned a lot through this $\mathrm{PhD}$ training. I have been changed, of course to the good direction. Although sometimes they were tough to me, I still appreciate a lot.

I should appreciate a lot to André. You help me really a lot. When I was in office, I always like to knock your door and ask you question directly. Thanks very much to you for tolerating my rude behavior and offer kind assistance. We have been several nice business trips in Beijing, Zhengzhou and Shanghai. You also give so much help on the research and I learned a lot from you. Besides that, you give me main support to finish my $\mathrm{PhD}$.

I would give my appreciation to Prof. Piet Dijksta during last period of finishing thesis. I have picked hope and started to treat writing work more carefully. You teach me a lot on writing and communication. Thanks!

I express my appreciation to all committee members, Prof. Dr. D. Stamatialis, Prof. Dr. G.J. Vancso, Dr. J.C.H. Leijten, Prof. Dr. J. Seppälä, Prof. Dr. J.L. Herek, Thank you very much participation my $\mathrm{PhD}$ defense.

I hearby thank Prof. Dr. Huiping Yuan, I indeed learn a lot clinic application knowledge from you and histology skills. Dr. Hubert, thank you very much for your collaboration and we had paper together.

I have so many colleagues and office mate to appreciate. Karin, you are always smile and would like to give your hand to me. Thanks! Marc, we had really a lot of talking, discussion, drinking and smoking. I always like to find you if I have problems in the lab. You are indeed a good 
person. I also need to thank Zlata, Lydia, Anita and Hetty for all your helps during my stay. Of course, in our group, I meet so many $\mathrm{PhD}$ colleagues, I do need to thank Seb who give me instruction in the beginning of my study. My office mate Natalia, Kasia, Dooli. I am so lucky to stay with you in one office. I thank Mike, Bas, Thijs, Ilaria, Odyl, Jonas. I am so happy to talk to you every time. Especially, my lab member, Bas, Mike and Thijs, I learned a lot from you, and I appreciate a lot your hands in the lab. I also enjoy the time we were going out to the restaurant and drinking outside of the building during summer.

I would like to thank my students Stijn and Sandra. You participated a lot of work in the thesis. I enjoy the time working together with you.

I want to thank so many Chinese friends here. Rong Wang, Honglin Chen, Yiyuan Lin, Siyuan Deng, Xiaohua Li, Kenan Niu, Yuxin Zhou, Jun Wang, Yao Fu, Rongquan Duan, Jia Song, Shaochen Zhu, Ye Xiang, Quan Wang, Xingwu Sun, Qirong Yao, Yunlong Yu, Youwen Fan, Mengdi Yang, Lantian Chang, Yin Du, Yang Wang, Yitong Zhou, Tong Wang, Xing An, Zhizhen Qin etc. Thanks, I am lucky to meet you all.

I would like to appreciate Jia Liang a lot. Thank you accompany all the time before and during my studying for $\mathrm{PhD}$.

In the end, I give my deep appreciation to my family, my father and mother, my sister. You give me support all the time and you are proud of me. Thank you!

Thank you all and best wishes,

Zhengchao Guo 
About the author:

Zhengchao Guo was born in 1988, Anyang, Henan province, China. He was obtained the bachelor's degree from Henan University of Technology in 2011. After that, he had been studying as master student in Zhengzhou University until 2014. He started his $\mathrm{PhD}$ from September 2014 in University of Twente in Enschede. The research filed is about processing polymeric materials and composites to be structure and implants for tissue engineering and regeneration. The important results during $\mathrm{PhD}$ studying were described in the thesis. 
ISBN: 978-90-365- 5074-1

DOI: 10.3990/1.9789036550741 